\title{
Topological logarithmic structures
}

\author{
JOHN ROGNES
}

\begin{abstract}
We develop a theory of logarithmic structures on structured ring spectra, including constructions of logarithmic topological André-Quillen homology and logarithmic topological Hochschild homology.
\end{abstract}

14F10, 55P43; 13D03, 55P47

\section{Introduction}

\subsection{Logarithmic algebraic geometry}

A logarithmic structure on a commutative ring $A$ is a commutative monoid $M$ with a homomorphism to the underlying multiplicative monoid of $A$. This determines a localization $A\left[M^{-1}\right]$ of $A$. In algebro-geometric terms, we might say that $M$ cuts out a divisor $D$ from $\operatorname{Spec}(A)$, and $A\left[M^{-1}\right]$ is the ring of regular functions on the open complement. In general the logarithmic structure carries more information than the localization. For example, the Kähler differentials of $A$ form an $A$-module $\Omega_{A}^{1}$, generated by differentials of the form $d a$, which are globally defined over $\operatorname{Spec}(A)$. The Kähler differentials of the localization form the $A\left[M^{-1}\right]$-module $\Omega_{A\left[M^{-1}\right]}^{1}$, which also contains differentials of the form $m^{-1} d a$, having poles of arbitrary degree along $D$. The logarithmic structure specifies an intermediate $A$-module of logarithmic Kähler differentials, $\Omega_{(A, M)}^{1}$, generated by differentials of the form $d a$ and $d \log m=m^{-1} d m$, having only poles of simple, or logarithmic, type along $D$. The logarithmic structure is therefore a more moderate way of specifying a localization than the actual localized ring. See Kato [35] and Illusie [34] for introductions to logarithmic algebraic geometry.

\subsection{Algebraic $K$-theory of rings and $S$-algebras}

We wish to apply the ideas of logarithmic geometry to the study of the algebraic $K$-theory of structured ring spectra, also known as commutative $S$-algebras. Typical examples of commutative $S$-algebras are the sphere spectrum $S$, the spherical group ring of the integers $S[\mathbb{Z}]$, the complex bordism spectrum $M U$, the complex $K$-theory spectrum $K U$ and the Eilenberg-Mac Lane spectrum $H R$ of a commutative ring $R$. 
Modern foundations are discussed in Elmendorf-Kriz-Mandell-May [21], HoveyShipley-Smith [32] and Schwede [73]. In the two first examples, $\mathrm{K}(S)=A(*)$ and $\mathrm{K}(S[\mathbb{Z}])=A\left(S^{1}\right)$ agree with Waldhausen's algebraic $K$-theory of the spaces $*$ and $S^{1}$, respectively, which are closely related to the diffeomorphism groups of highdimensional manifolds. More precisely, $A(*)$ and $A\left(S^{1}\right)$ determine the stable smooth pseudoisotopy spaces of $*$ and $S^{1}$, respectively, and these in turn determine the stable smooth pseudoisotopy spaces of all closed nonpositively curved Riemannian manifolds, via their points and closed geodesics. See Waldhausen [80], Farrell-Jones [22] and Waldhausen-Jahren-Rognes [81].

In the third example, $\mathrm{K}(M U)$ remains mysterious, but appears to be an interesting halfway house between the earlier and the later examples. A key step towards its determination is the homotopy limit property for cyclic group actions on its topological Hochschild homology THH(MU), which has been established by Lunøe-Nielsen and Rognes in [43]. In the fourth example, $\mathrm{K}(K U)$ classifies virtual 2-vector bundles, and is related to a form of elliptic cohomology. See Ausoni-Rognes [5], [6], BaasDundas-Rognes [10] and Baas-Dundas-Richter-Rognes [8], [9]. In the fifth example, $\mathrm{K}(H R)=\mathrm{K}(R)$ agrees with Quillen's algebraic $K$-theory, and when $R$ is a local or global number ring, this captures a great deal of the arithmetic, or number-theoretic, invariants of that number ring. See Quillen [61], Dwyer-Friedlander-Snaith-Thomason [20] and Rognes-Weibel [66], plus the work of Voevodsky and Rost on the Milnorand Bloch-Kato conjectures.

We would like to understand the algebraic $K$-theory of commutative $S$-algebras in the same kind of conceptual terms as we understand the algebraic $K$-theory of number rings. This includes the principles that algebraic $K$-theory satisfies étale descent and localization properties, with certain modifications, like a restriction to finite coefficients and sufficiently high degrees in the case of étale descent. See Quillen [62] and Thomason-Trobaugh [78]. Two approaches have been successful in proving that algebraic $K$-theory is close to satisfying étale descent. One is based on Voevodsky's motivic cohomology and its relation to étale cohomology, as explained by the Milnorand Bloch-Kato conjectures just mentioned. However, this theory depends to some extent on resolution of singularities, and any theory that is hard to extend to rings of positive characteristic will also be hard to extend to commutative $S$-algebras.

\subsection{Topological cyclic homology and the de Rham-Witt complex}

The other approach is based on the cyclotomic trace map from algebraic $K$-theory to the topological cyclic homology of Bökstedt-Hsiang-Madsen [15]. This is the tool of choice for the study of the $p$-adically completed algebraic $K$-theory of a $p$-complete 
ring, and more generally, for a connective, $p$-complete commutative $S$-algebra $A$, since it is a natural map

$$
\text { trc: } \mathrm{K}(A) \rightarrow \mathrm{TC}(A ; p)
$$

(of spaces, say, for simplicity), that becomes a homotopy equivalence after $p$-adic completion whenever $\pi_{0}(A)$ is a finite algebra over the Witt vectors of a perfect field $k$ of characteristic $p$. See Hesselholt-Madsen [28]. This approach suffices for the determination of the $p$-complete algebraic $K$-theory $\mathrm{K}(A)_{p}$ in some cases, such as the sphere spectrum $A=S$ when $p$ is a regular prime. See Rognes [64; 65]. Furthermore, there is a very close relationship between the topological cyclic homology of a commutative ring $A$ and the de Rham-Witt complex $W_{\bullet} \Omega_{A}^{*}$, which is built upon the de Rham complex $\Omega_{A}^{*}$ given by the exterior algebra on the Kähler differentials $\Omega_{A}^{1}$ that we started with. See Illusie [33] and Hesselholt [27]. The relationship is the closest when $A$ is a smooth algebra over the perfect field $k$.

The condition that $A$ is connective and $p$-complete of suitably finite type is almost orthogonal to our desire to understand the algebraic $K$-theory of commutative $S$ algebras in terms of étale descent and localization properties. For étale descent involves the formation of homotopy limits, specializing to the formation of homotopy fixed points in the case of Galois descent, and such limits often take us out of the category of connective spectra. Similarly, localization of a $p$-complete ring by inverting $p$ will give a rational algebra, whose $p$-completion is trivial, leaving no information to be seen by topological cyclic homology. Furthermore, localization of a commutative $S$-algebra by inverting a positive-dimensional element, or by more general Bousfield localizations, will most often give a nonconnective result.

\subsection{Algebraic $K$-theory of local fields}

In the context of discrete valuation rings, Hesselholt-Madsen [29] overcome this difficulty by the use of logarithmic structures and logarithmic differentials. We illustrate by their main example. Let $K$ be a $p$-adic number field, ie, a finite extension of $\mathbb{Q}_{p}$, let $A \subset K$ be its valuation ring, and let $k$ be the residue field. The maximal ideal of $A$ is generated by an uniformizer $\pi$, so that $K=A\left[\pi^{-1}\right]$ and $k=A /(\pi)$. There is a localization sequence

$$
\mathrm{K}(k) \stackrel{i_{*}}{\rightarrow} \mathrm{K}(A) \stackrel{j^{*}}{\rightarrow} \mathrm{K}(K)
$$

in Quillen $K$-theory [61]. Here $i_{*}$ is the transfer map (= direct image) associated to the surjection $i: A \rightarrow k$, we write $j^{*}$ for the natural map (=inverse image) associated to the inclusion $j: A \rightarrow K$, and the sequence is a homotopy fiber sequence of spaces. The cyclotomic trace maps $\mathrm{K}(k) \rightarrow \mathrm{TC}(k ; p)$ and $\mathrm{K}(A) \rightarrow \mathrm{TC}(A ; p)$ are $p$-adic equivalences of spaces, as explained above, so Hesselholt and Madsen construct a 
relative form of topological cyclic homology, denoted $\mathrm{TC}(A \mid K ; p)$, that sits in a similar homotopy fiber sequence

$$
\mathrm{TC}(k ; p) \stackrel{i_{*}}{\rightarrow} \mathrm{TC}(A ; p) \stackrel{j^{*}}{\rightarrow} \mathrm{TC}(A \mid K ; p)
$$

(where $\operatorname{TC}(A \mid K ; p)$ is not the same as the $p$-adically trivial $\operatorname{TC}(K ; p)$ ). Much as the de Rham-Witt complex $W_{\bullet} \Omega_{A}^{*}$ is built on top of the de Rham complex $\Omega_{A}^{*}$, the topological cyclic homology $\mathrm{TC}(A ; p)$ is built on top of the topological Hochschild homology $\operatorname{THH}(A)$. So the homotopy fiber sequence above is in fact extracted from a homotopy fiber sequence

$$
\mathrm{THH}(k) \stackrel{i_{*}}{\rightarrow} \mathrm{THH}(A) \stackrel{j^{*}}{\rightarrow} \mathrm{THH}(A \mid K)
$$

of so-called cyclotomic spectra. The Hesselholt-Madsen construction of $\operatorname{THH}(A \mid K)$ does a priori not have anything to do with logarithmic geometry, but under these hypotheses, they are able to compute the homotopy of $\operatorname{THH}(A \mid K)$ and $\operatorname{TC}(A \mid K ; p)$ (with mod $p$ coefficients), and to express the answers in terms of a logarithmic de RhamWitt complex $W_{\bullet} \Omega_{(A, M)}^{*}$ associated to the valuation ring $A$ with the logarithmic structure given by the multiplicative monoid $M=A \cap K^{*}$ of nonzero elements in $A$. The first sign of this is seen in the long exact sequence in homotopy associated to the latter homotopy fiber sequence, which contains the extension

$$
0 \rightarrow \Omega_{A}^{1} \stackrel{\pi_{1}\left(j^{*}\right)}{\longrightarrow} \Omega_{(A, M)}^{1} \stackrel{\text { res }}{\longrightarrow} k \rightarrow 0
$$

in dimensions 1 and 0 . The first map is the inclusion of Kähler forms among logarithmic Kähler forms, while the residue map res takes $d \log \pi$ to 1 and is realized as the connecting map in the long exact sequence. For THH, the result is that

$$
\pi_{*}(\operatorname{THH}(A \mid K) ; \mathbb{Z} / p) \cong \Omega_{(A, K)}^{*} \otimes \mathbb{Z} / p\left[\kappa_{0}\right]
$$

where $\left|\kappa_{0}\right|=2$. The algebraic theory of logarithmic de Rham-Witt complexes is developed further in Hesselholt-Madsen [30] and Langer-Zink [38]. The passage from $\mathrm{TC}(A ; p)$ to $\operatorname{TC}(A \mid K ; p)$ is an essential step to make these calculations manageable. As a consequence of the calculations, one sees that $\mathrm{TC}(A \mid K ; p)$ satisfies descent for Galois extensions $K \rightarrow L$ to the extent expected for algebraic $K$-theory. This is obscure, at best, in the comparison of $\operatorname{TC}(A ; p)$ with $\operatorname{TC}(B ; p)$, where $B$ is the valuation ring of $L$. Hence $\mathrm{TC}(A \mid K ; p)$ and $W_{\bullet} \Omega_{(A, M)}^{*}$ are essential ingredients in the Hesselholt-Madsen proof of Galois descent for $p$-completed $\mathrm{K}(K)$, and étale descent for $p$-completed $\mathrm{K}(A)$, for these local fields and rings. 


\subsection{Algebraic contents of the present paper}

In this paper we give a sense to $\operatorname{THH}(A, M)$ for general commutative rings with logarithmic structure $(A, M)$, as a cyclic commutative $A$-algebra. We expect that

$$
\operatorname{THH}(A, M) \simeq \operatorname{THH}(A \mid K)
$$

in all cases when the right hand side is defined, but we only prove that the mod $p$ homotopy algebras are isomorphic. We give two equivalent constructions of $\operatorname{THH}(A, M)$. The first is given in Definition 8.11 in terms of the replete bar construction $B^{\text {rep }} M$ of $M$, and the repletion map

$$
B^{\text {cy }} M \rightarrow B^{\text {rep }} M
$$

from the usual cyclic bar construction. The second is given in Definition 13.10, in terms of the suspension in the category of augmented commutative $S[M]$-algebras of a shear map

$$
\text { sh: } S[M] \wedge S[M] \rightarrow S[M] \wedge S\left[M^{\mathrm{gp}}\right],
$$

symbolically given by $(x, y) \mapsto(x y, \gamma(y))$. Here $\gamma: M \rightarrow M^{\text {gp }}$ is the group completion homomorphism. The comparison of $\operatorname{THH}(A, M)$ with $\operatorname{THH}(A \mid K)$ is discussed in Example 8.14 and Proposition 8.15.

In this paper we also give a sense to the logarithmic topological André-Quillen homology $\operatorname{TAQ}(A, M)$ for logarithmic rings $(A, M)$, as an $A$-module. The relation of $\operatorname{THH}(A, M)$ to $\operatorname{TAQ}(A, M)$ is like that of the logarithmic de Rham complex $\Omega_{(A, M)}^{*}$ to the logarithmic Kähler differentials $\Omega_{(A, M)}^{1}$, especially in the smooth, or logarithmically smooth, cases. We review the ordinary theory of topological André-Quillen homology in Section 10, give one construction of $\operatorname{TAQ}(A, M)$ in Definition 11.19 and give a second, equivalent, construction in Definition 13.13. The latter is expressed in terms of the infinite suspension of the shear map, in the category of augmented commutative $S[M]$-algebras.

We approach these definitions in several stages, to motivate and justify them. First we think of Kähler differentials as corepresenting derivations, and we follow Quillen [60] in thinking of derivations as homomorphisms into abelian group objects. This leads us to consider abelian group objects in suitable categories of logarithmic rings, to define logarithmic derivations as morphisms in these categories and to construct logarithmic Kähler differentials as corepresenting objects for logarithmic derivations. This way we recover Kato's definition of $\Omega_{(A, M)}^{1}$ as a pushout of $A$-modules, in Definition 4.25. In Section 5 we make a corresponding analysis for "associative ring maps between commutative rings", which leads to a definition of logarithmic Hochschild homology $\mathrm{HH}(A, M)$ as a pushout of commutative $A$-algebras, with a comparison map 
$\Omega_{(A, M)}^{*} \rightarrow \mathrm{HH}_{*}(A, M)$. The analogous discussion for logarithmic $S$-algebras leads to the initial definitions of the corepresenting objects $\operatorname{TAQ}(A, M)$ and $\operatorname{THH}(A, M)$, as suitable homotopy pushouts.

In Section 3 we argue that certain features of the traditional algebro-geometric theory of logarithmic rings, namely that one works within the full subcategory of so-called fine and saturated logarithmic rings, can constructively be replaced by a different condition that is better suited for topological generalization. The alternate condition is a relative one, ie, it is a condition on logarithmic rings $(A, M)$ over a fixed base logarithmic ring $(R, P)$, and asks that the monoid homomorphism $M \rightarrow P$ to the base commutative monoid is an exact surjection. Here exactness means that $M \rightarrow P$ is the pullback of $M^{\mathrm{gp}} \rightarrow P^{\mathrm{gp}}$ along $\gamma: P \rightarrow P^{\mathrm{gp}}$. We say that such logarithmic rings $(A, M)$ are replete over $(R, P)$, and we prove in Lemma 3.8 (with a topological analogue in Proposition 8.3) that there is a repletion functor for quite general logarithmic rings $(A, M)$ over $(R, P)$. The repletion of the cyclic bar construction $B^{\text {cy }} M$ of a commutative monoid is, by definition, the replete bar construction $B^{\text {rep }} M$, and the cyclic structure on $B^{\text {cy }} M$ carries over to a cyclic structure on $B^{\text {rep }} M$. This leads to a revised Definition 8.11 of $\operatorname{THH}(A, M)$, in terms of repletion. Its advantage over the previous characterization is that $\operatorname{THH}(A, M)$ now is a cyclic object in commutative $A$-algebras, which is a first step towards a cyclotomic structure.

In a third and final iteration, we note that the repletion map required for the definition of $\operatorname{TAQ}(A, M)$ is the infinite stabilization of the repletion map required for $\operatorname{THH}(A, M)$, and that this in turn is a single stabilization of a shear map $A \wedge S[M] \rightarrow A \wedge S\left[M^{\mathrm{gp}}\right]$ in the category of augmented commutative $A$-algebras. We are therefore able to give quite short and direct definitions of the logarithmic Hochschild homology $\mathrm{HH}(A, M)$, higher order versions $\mathrm{HH}^{[n]}(A, M)$ and their stabilization $H \Gamma(A, M)$ as $n \rightarrow \infty$, in Section 13. These give logarithmic forms of constructions of Pirashvili [56] and Robinson-Whitehouse [63]. This all adds to the belief that for each logarithmic ring $(A, M)$, the stable category of spectra formed in the category of simplicial replete logarithmic rings under and over $(A, M)$ will be an appropriate category of logarithmic $(A, M)$-modules. Note that working in the subcategory of replete logarithmic rings replaces all nonempty colimits (= tensor products) formed in logarithmic rings by their repletions, hence the tensored structure and suspension in simplicial replete logarithmic rings will be different from those in simplicial logarithmic rings. See Remark 4.14 for some further discussion.

\subsection{Algebraic $K$-theory of topological $K$-theory}

Moving on from discrete rings to commutative $S$-algebras, the examples that are the closest to algebra are given by the topological $K$-theory spectrum $K U$ and its 
variants. Let $K U_{p}$ be its $p$-completion, let $L_{p}$ be the Adams summand of $K U_{p}$, and let $\ell_{p}$ and $k u_{p}$ be the respective connective covers. These are all commutative $S$-algebras. The $\bmod p$ reductions $L / p=K(1), K U / p, \ell / p=k(1)$ and $k u / p$ are associative $S$-algebras, but not commutative $S$-algebras. The $p$-complete algebraic $K$-theory of $\ell_{p}, k u_{p}$ and $\ell / p$ was computed in Ausoni-Rognes [5], Ausoni [3] and Ausoni-Rognes [7], respectively, in each case using the equivalence with topological cyclic homology. For simplicity, we focus on the Adams summand cases. There are localization sequences

$$
\begin{gathered}
\mathrm{K}\left(\mathbb{Z}_{p}\right) \stackrel{\pi_{*}}{\longrightarrow} \mathrm{K}\left(\ell_{p}\right) \stackrel{\rho^{*}}{\rightarrow} \mathrm{K}\left(L_{p}\right) \\
\mathrm{K}(\mathbb{Z} / p) \stackrel{\pi_{*}}{\longrightarrow} \mathrm{K}(\ell / p) \stackrel{\rho^{*}}{\longrightarrow} \mathrm{K}(L / p)
\end{gathered}
$$

established by Blumberg and Mandell [14]. Here $\pi_{*}$ denotes the transfer maps associated to the 1 -connected maps $\pi: \ell_{p} \rightarrow H \mathbb{Z}_{p}$ and $\pi: \ell / p \rightarrow H \mathbb{Z} / p$, and $\rho^{*}$ denotes the natural map associated to the localization maps $\rho: \ell_{p} \rightarrow L_{p}$ and $\rho: \ell / p \rightarrow L / p$. The cyclotomic trace map $K(A) \rightarrow \mathrm{TC}(A ; p)$ is a $p$-adic equivalence in all of the cases $A=H \mathbb{Z}_{p}, \ell_{p}, H \mathbb{Z} / p$ and $\ell / p$, but not for the nonconnective spectra $A=L_{p}$ and $L / p$. We would therefore like to construct relative forms of topological cyclic homology, denoted $\operatorname{TC}\left(\ell_{p} \mid L_{p} ; p\right)$ and $\operatorname{TC}(\ell / p \mid L / p ; p)$, that sit in homotopy fiber sequences

$$
\begin{gathered}
\mathrm{TC}\left(\mathbb{Z}_{p} ; p\right) \stackrel{\pi_{*}}{\longrightarrow} \mathrm{TC}\left(\ell_{p} ; p\right) \stackrel{\rho^{*}}{\longrightarrow} \mathrm{TC}\left(\ell_{p} \mid L_{p} ; p\right) \\
\mathrm{TC}(\mathbb{Z} / p ; p) \stackrel{\pi_{*}}{\longrightarrow} \mathrm{TC}(\ell / p ; p) \stackrel{\rho^{*}}{\longrightarrow} \mathrm{TC}(\ell / p \mid L / p ; p)
\end{gathered}
$$

and are extracted by a limiting process from homotopy fiber sequences

$$
\begin{aligned}
\operatorname{THH}\left(\mathbb{Z}_{p}\right) \stackrel{\pi_{*}}{\longrightarrow} \operatorname{THH}\left(\ell_{p}\right) \stackrel{\rho^{*}}{\longrightarrow} \operatorname{THH}\left(\ell_{p} \mid L_{p}\right) \\
\operatorname{THH}(\mathbb{Z} / p) \stackrel{\pi_{*}}{\longrightarrow} \operatorname{THH}(\ell / p) \stackrel{\rho^{*}}{\longrightarrow} \operatorname{THH}(\ell / p \mid L / p)
\end{aligned}
$$

of cyclotomic spectra. The Hesselholt-Madsen construction of $\operatorname{THH}(A \mid K)$ for discrete valuation rings does not easily generalize to this topological setting of structured ring spectra, so we seek instead to generalize the construction of $\operatorname{THH}(A, M)$ to this topological setting, so as to realize $\operatorname{THH}\left(\ell_{p} \mid L_{p}\right)$, and perhaps $\operatorname{THH}(\ell / p \mid L / p)$, in that form.

\subsection{Topological contents of the present paper}

We expect this algebro-geometric theory to be most useful in the commutative context, where we replace the commutative ring $A$ by a commutative $S$-algebra. Experience 
from structured ring spectrum theory tells us that we should replace the commutative monoid $M$ by the kind of space that arises as the underlying space of a commutative $S$-algebra, with its multiplicative structure. These are, a little informally, known as $E_{\infty}$ spaces. More precisely, when commutative $S$-algebras are interpreted in the sense of Elmendorf-Kriz-Mandell-May [21], they are $\mathcal{L}$-spaces where $\mathcal{L}$ is the linear isometries operad, and when commutative $S$-algebras are interpreted in the sense of Hovey-Shipley-Smith [32], they are commutative $\mathcal{I}$-space monoids. A logarithmic structure on a commutative $S$-algebra $A$ is then an $E_{\infty}$ space $M$ with an $E_{\infty}$ map to the underlying multiplicative $E_{\infty}$ space $\Omega_{\otimes}^{\infty} A$ of $A$. In fact, the underlying multiplicative spaces of commutative $S$-algebras are somewhat special $E_{\infty}$ spaces, because the additive base point of the underlying space acts as a zero, or base point, for the multiplication. This leads us to work with based $E_{\infty}$ spaces, also known as $E_{\infty}$ spaces with zero. A further justification for working with based $E_{\infty}$ spaces is illustrated by thinking of $L_{p}$ as the localization of $\ell_{p}$ obtained by inverting the element $v_{1} \in \pi_{*} \ell_{p}=\mathbb{Z}_{p}\left[v_{1}\right]$. Let $f: S^{q} \rightarrow \Omega_{\otimes}^{\infty} \ell_{p}$ be a map representing $v_{1}$ in homotopy, where $q=2 p-2$. It is not the individual multiplication maps $f(z) \cdot: \ell_{p} \rightarrow \ell_{p}$, for $z \in S^{q}$, that become equivalences after base change to $L_{p}$, but the combined multiplication map $f \cdot: S^{q} \wedge \ell_{p} \rightarrow \ell_{p}$. To induce this map from the smash product, $f$ must be thought of as a base-point preserving map. When we extend $f$ to a multiplicative map, that must be a map of based $E_{\infty}$ spaces.

We define based and unbased topological logarithmic structures in Section 7, after discussing the available choices of technical foundations in Section 6. The definitions of logarithmic topological Hochschild homology given in Sections 8 and 13 immediately generalize from the case of a discrete commutative monoid $M$ to the case of an unbased $E_{\infty}$ space $M$, and similarly for the definitions of logarithmic topological André-Quillen homology in Sections 11 and 13. However, for based $E_{\infty}$ spaces $N$ we need to make a topological assumption about the local structure near the base point, namely that the based $E_{\infty}$ space is conically based; see Definition 6.21. This ensures that the spaces of based logarithmic derivations are corepresentable, as in Lemmas 12.3 and 12.4, and lets us define a based logarithmic topological André-Quillen homology $\mathrm{TAQ}_{0}(A, N)$ in Definition 12.6, with a companion definition of based logarithmic topological Hochschild homology $\mathrm{THH}_{0}(A, N)$ in Definition 8.17. For conically based $E_{\infty}$ spaces $N$ the base point complement $N^{\prime}$ is an $E_{\infty}$ space, there is a conical diagonal map $\delta: N \rightarrow N \wedge N_{+}^{\prime}$, and there is a based shear map

$$
\text { sh: } \Sigma^{\infty} N \wedge \Sigma^{\infty} N \rightarrow \Sigma^{\infty} N \wedge S\left[N^{\prime}\right]
$$

of augmented commutative $\Sigma^{\infty} N$-algebras. In Section 13 we give streamlined definitions of based logarithmic topological Hochschild homology $\mathrm{THH}_{0}(A, N)$ as a cyclic 
commutative $A$-algebra and based logarithmic topological André-Quillen homology $\mathrm{TAQ}_{0}(A, N)$ as an $A$-module, using single and infinite suspensions of this based shear map.

\subsection{Logarithmic structures on topological $K$-theory}

Returning to the desired logarithmic model $\mathrm{TC}\left(\ell_{p} \mid L_{p} ; p\right)$ for the $p$-completed $\mathrm{K}\left(L_{p}\right)$, we seek a based logarithmic structure $N$ on $\ell_{p}$ so that

$$
\operatorname{THH}\left(\ell_{p} \mid L_{p}\right) \simeq \operatorname{THH}_{0}\left(\ell_{p}, N\right) .
$$

We have not yet been able to find a suitable $N$ in the current set-up, but some partial information is available, which we discuss in this paper. First, the periodic Adams summand $L_{p}$ is obtained from $\ell_{p}$ by inverting the map $v_{1} \cdot: \Sigma^{q} \ell_{p} \rightarrow \ell_{p}$, given by multiplication by a map $f: S^{q} \rightarrow \Omega^{\infty} \ell_{p}$ that represents $v_{1} \in \pi_{*} \ell_{p}$. Letting

$$
N \simeq \bigvee_{j \geq 0} S_{h \Sigma_{j}}^{q j}
$$

be the free based $E_{\infty}$ space generated by $S^{q}$, we obtain a based (pre-)logarithmic structure $N \rightarrow \Omega_{\otimes}^{\infty} \ell_{p}$. In view of the calculations by Bökstedt (unpublished) and McClure-Staffeldt [49] (see also Ausoni-Rognes [5]), the homotopy of $\operatorname{THH}\left(\mathbb{Z}_{p}\right)$ and $\operatorname{THH}\left(\ell_{p}\right)$ with coefficients in the Smith-Toda complex $V(1)=S /\left(p, v_{1}\right)$ is known, and this lets us compute the desired homotopy of $\operatorname{THH}\left(\ell_{p} \mid L_{p}\right)$. We have not calculated $\mathrm{THH}_{0}\left(\ell_{p}, N\right)$ in this case, but related calculations in the context of $\mathrm{TAQ}_{0}$ (see Examples 12.16 and 12.17) show that this free based $E_{\infty}$ space $N$ is not the desired based $E_{\infty}$ space. There may be a better based $E_{\infty}$ space, built from $N$ by attaching further free based $E_{\infty}$ cells, but this remains to be determined.

We now discuss two alternative approaches to this problem. The first involves working with less commutative ring spectra than $E_{\infty}$ ring spectra. Algebraic $K$-theory, topological cyclic homology and topological Hochschild homology are all defined for associative $S$-algebras, or $A_{\infty}$ ring spectra, but the question is how much commutativity is needed to make good sense of a logarithmic geometry. If we take

$$
N \simeq \bigvee_{j \geq 0} S^{q j}
$$

to be the free based $A_{\infty}$ space generated by $S^{q}$, we can extend the scope of based logarithmic topological Hochschild homology to make sense of $\mathrm{THH}_{0}\left(\Sigma^{\infty} N, N\right)$. With some more commutativity in $N$, so that $\operatorname{THH}\left(\Sigma^{\infty} N\right)$ is an associative $S$ algebra, this lets us make sense of $\operatorname{THH}_{0}\left(\ell_{p}, N\right)$, in such a way that $\operatorname{THH}\left(\ell_{p} \mid L_{p}\right) \simeq$ 
$\mathrm{THH}_{0}\left(\ell_{p}, N\right)$. In Section 9 we discuss evidence that $N$ has a based $E_{2}$ space structure, related to braid groups, which would suffice for this purpose.

The second approach involves working with commutative $M U$-algebras in place of commutative $S$-algebras. With $N \simeq \bigvee_{j \geq 0} S^{q j}$, as above, the suspension spectrum $\Sigma^{\infty} N$ is an associative but not a commutative $S$-algebra. However, its base change

$$
M U \wedge N \simeq \bigvee_{j \geq 0} \Sigma^{q j} M U
$$

to $M U$ is a commutative $M U$-algebra. This can be seen by a geometric construction similar to that of Neil Strickland [77, Appendix A]. There is then a commutative $M U$-algebra map $M U \wedge N \rightarrow \ell_{p}$, which we view as specifying a complex oriented logarithmic structure, that permits us to define a cyclic commutative $M U$-algebra model for $\operatorname{THH}\left(\ell_{p} \mid L_{p}\right)$. More generally, this works to define $\operatorname{THH}(e \mid E)$ for periodic commutative $M U$-algebras $E$ with connective cover $e$. For the purpose of using logarithmic geometry to bridge the gap from $K(M U)$ to $K(\mathbb{Z})$, this appears to be a viable route. For time reasons we are unable to include the discussion of complex oriented logarithmic structures in this paper, but we plan to return to it in a later publication.

\subsection{The fraction field of topological $K$-theory}

The calculations of Ausoni-Rognes [7] show that the diagrams

$$
\begin{gathered}
\mathrm{K}(\ell / p) \stackrel{i_{*}}{\rightarrow} \mathrm{K}\left(\ell_{p}\right) \stackrel{j^{*}}{\rightarrow} \mathrm{K}\left(\ell \mathbb{Q}_{p}\right) \\
\mathrm{K}(L / p) \stackrel{i_{*}}{\rightarrow} \mathrm{K}\left(L_{p}\right) \stackrel{j^{*}}{\rightarrow} \mathrm{K}\left(L \mathbb{Q}_{p}\right)
\end{gathered}
$$

are not homotopy fiber sequences. Here $\ell \mathbb{Q}_{p}=\ell_{p}[1 / p]$ and $L \mathbb{Q}_{p}=L_{p}[1 / p]$ are the commutative $H \mathbb{Q}_{p}$-algebra spectra that are obtained by inverting $p$ in $\ell_{p}$ and $L_{p}$, respectively. Indeed, the calculations essentially show that the mapping cones of the two transfer maps $i_{*}$, which we denote $K(\mathcal{O})$ and $K(\mathcal{F})$ for brevity, have large $v_{2}$-periodic $V(1)$-homotopy, whereas $K\left(\mathbb{Q}_{p}\right)$ and any algebra over it is $v_{2}$-torsion. With this notation, we have a $3 \times 3$ square of homotopy fiber sequences

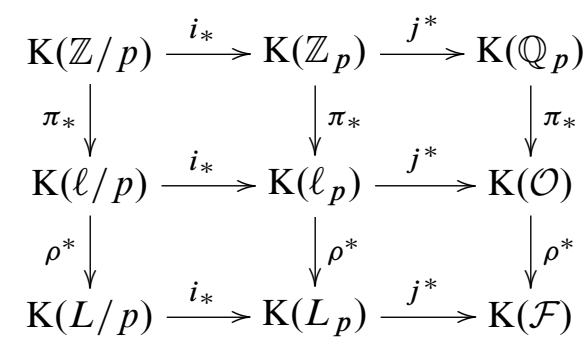


where the $V(1)$-homotopy of all four corners in the upper left hand square have been computed using topological cyclic homology.

This leads to the question of what kind of objects $\mathcal{O}$ and $\mathcal{F}$ are. Considering the lower row, we view $\mathcal{F}$ as a milder localization of $L_{p}$ away from its noncommutative residue $S$-algebra $L / p$ than the algebraic localization that inverts $p$. Since this is the only nontrivial residue $S$-algebra of $L_{p}$, we think of $\mathcal{F}$ as specifying an $S$-algebraic fraction field of $L_{p}$, and $\mathcal{O}$ as a connective valuation $S$-algebra of $\mathcal{F}$. Furthermore, it appears that the Galois cohomology of this object $\mathcal{F}$, with $V(1)$-coefficients, is a Poincaré duality algebra with fundamental class in $H_{\text {Gal }}^{3}\left(\mathcal{F} ; \mathbb{F}_{p^{2}}(2)\right)$, indicating that $\mathcal{F}$ is a kind of $S$-algebraic higher local field satisfying arithmetic duality as discussed in Milne [50]. We explain these calculations in greater detail in Ausoni-Rognes [7].

Given the thrust of the present paper, it should come as no surprise that we expect to be able to realize $\mathcal{O}$ and $\mathcal{F}$ as logarithmic $S$-algebras, so that there should be based $E_{\infty}$ spaces $S^{0}=\{0,1\},\left\langle v_{1}\right\rangle,\langle p\rangle$ and $\left\langle p, v_{1}\right\rangle$ mapping to $\Omega_{\otimes}^{\infty} \ell_{p}$, so that the lower right hand square above is realized as the algebraic $K$-theory of the commutative square

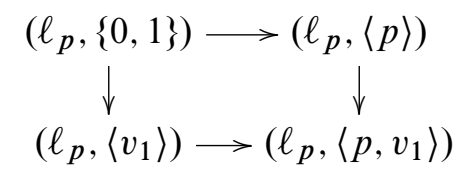

of based logarithmic $S$-algebras. A similar triple division of approaches arises as in the discussion of logarithmic structures on (the Adams summand of) topological $K$-theory, and again there is a discussion of homological obstructions and natural hypotheses in Section 9. As in that case, a complex oriented (pre-)logarithmic structure $M U \wedge\left\langle p, v_{1}\right\rangle \rightarrow \ell_{p}$ appears to be the best commutative model for the fraction field $\mathcal{F}$.

The $V(1)$-homotopy of the corresponding $3 \times 3$ square of homotopy fiber sequences

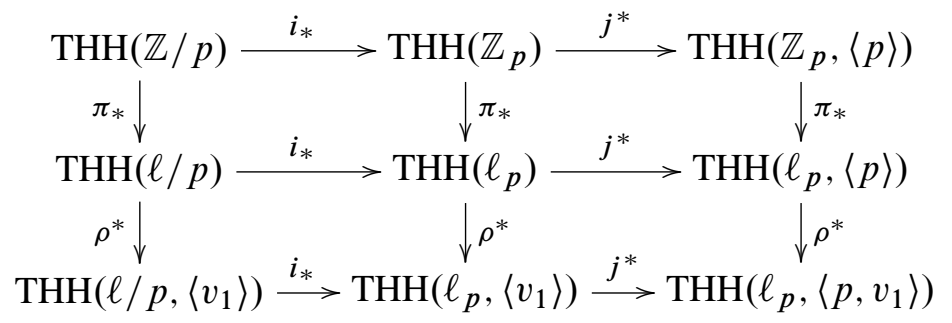

(as well as its analogue for TC) is computed in Ausoni-Rognes [7], starting from calculations of the upper left hand square. In the fraction field corner, the conclusion

$$
V(1)_{*} \operatorname{THH}\left(\ell_{p},\left\langle p, v_{1}\right\rangle\right) \cong E\left(d \log p, d \log v_{1}\right) \otimes \mathbb{Z} / p\left[\kappa_{0}\right]
$$


with $|d \log p|=\left|d \log v_{1}\right|=1$ and $\left|\kappa_{0}\right|=2$ is nicely compatible with the HesselholtMadsen result for $\pi_{*}(\mathrm{THH}(A \mid K) ; \mathbb{Z} / p)$.

Acknowledgments The author first learned from the work of Lars Hesselholt and $\mathrm{Ib}$ Madsen how differentials with logarithmic poles play a role in topological Hochschild homology, topological cyclic homology and $p$-adic algebraic $K$-theory. He would like to thank Clark Barwick for emphasizing how these logarithmic differentials stem from the underlying algebraic geometry of logarithmic schemes. Furthermore, he would like to thank Vigleik Angeltveit, Christian Ausoni, Marcel Bökstedt, Bob Bruner, Fred Cohen, Bill Dwyer, Thomas Kragh, Sverre Lunøe-Nielsen, Mike Mandell, Peter May, Haynes Miller, Martin C Olsson, Steffen Sagave and Christian Schlichtkrull for helpful communications related to this paper. Many thanks are also due to Andy Baker and Birgit Richter for organizing the Banff conference in March 2008 and for editing these proceedings.

The length of this paper is unfortunate. It stems from the desire to both discuss the algebraic background, where we can give explicit formulas for maps and study interesting examples, but also to reach the based topological structures that are required to describe the fraction field of topological $K$-theory. It also stems from the iterated approach to the definitions, starting from logarithmic derivations and their corepresenting logarithmic differentials, via the introduction of replete logarithmic structures, to the final quick definitions using the shear map. The author believes that a record of this transition has some value, even if later applications of logarithmic topological Hochschild homology may just start from the end. Still, the author feels that the reader deserves an apology for the length of the exposition.

\section{Part I Logarithmic structures on commutative rings}

\section{Commutative logarithmic structures}

We begin by reviewing some basic definitions about the log structures of Jean-Marc Fontaine and Luc Illusie, adapting Kazuya Kato's introduction [35, Section 1] to the affine case. For simplicity we work with commutative rings, but it is just about as easy to work with commutative $R$-algebras over some base commutative ring $R$.

Definition 2.1 Let $A$ be a commutative ring. A pre-log structure on $A$ is a pair $(M, \alpha)$ consisting of a commutative monoid $M$ and a monoid homomorphism

$$
\alpha: M \rightarrow(A, \cdot)
$$


to the underlying multiplicative monoid of $A$. A pre-log ring $(A, M, \alpha)$ is a commutative ring $A$ with a pre-log structure $(M, \alpha)$, often abbreviated to $(A, M)$. A homomorphism

$$
\left(f, f^{b}\right):(A, M, \alpha) \rightarrow(B, N, \beta)
$$

of pre-log rings consists of a ring homomorphism $f: A \rightarrow B$ and a monoid homomorphism $f^{b}: M \rightarrow N$, such that the square

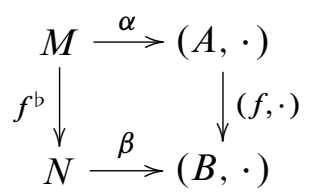

commutes. Here $(f, \cdot):(A, \cdot) \rightarrow(B, \cdot)$ denotes the underlying multiplicative monoid homomorphism of $f$. Pre-log rings and homomorphisms form a category, which we denote $\operatorname{Pr}$ r $\mathcal{L}$ og. There are obvious forgetful functors from $\mathcal{P}$ re $\mathcal{L}$ og to the categories $\mathcal{C R}$ ing and $\mathcal{C}$ Mon of commutative rings and commutative monoids, respectively.

Remark 2.2 Let $\mathbb{Z}[M]$ denote the monoid ring of $M$. The functor $M \mapsto \mathbb{Z}[M]$, from commutative monoids to commutative rings, is left adjoint to the functor $A \mapsto(A, \cdot)$.

$$
\text { CMon } \underset{(-, \cdot)}{\stackrel{\mathbb{Z}[-]}{\rightleftarrows}} \mathcal{C R i n g}
$$

Hence a pre-log structure $(M, \alpha)$ can equally well be defined in terms of the ring homomorphism $\bar{\alpha}: \mathbb{Z}[M] \rightarrow A$ that is left adjoint to $\alpha$. A pair $\left(f, f^{b}\right)$ of ring and monoid homomorphisms, respectively, then defines a pre-log homomorphism $(A, M) \rightarrow(B, N)$ if and only if the square

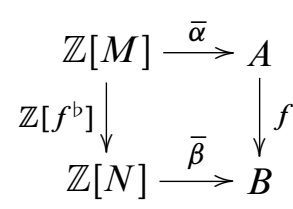

commutes.

Definition 2.3 Let $\iota: \mathrm{GL}_{1}(A) \subset(A, \cdot)$ denote the inclusion of the multiplicative group of units in $A$. Let $\alpha^{-1} \mathrm{GL}_{1}(A) \subset M$ be defined by the pullback square

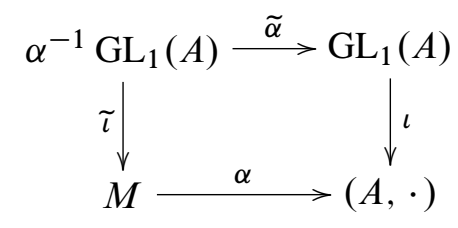


of commutative monoids. The pre-log structure $(M, \alpha)$ is a $\log$ structure on $A$ if the restricted homomorphism $\widetilde{\alpha}: \alpha^{-1} \mathrm{GL}_{1}(A) \rightarrow \mathrm{GL}_{1}(A)$ is an isomorphism. Log rings generate a full subcategory of $\operatorname{Pre} \log$, which we denote $\mathcal{L} o g$.

Remark 2.4 The forgetful functor $\mathcal{A} b \rightarrow \mathcal{C M}$ on, from abelian groups to commutative monoids, has a right and a left adjoint. The right adjoint takes a commutative monoid $M$ to its subgroup $M^{*}$ of units, while the left adjoint takes a commutative monoid $M$ to its group completion $M^{\mathrm{gp}}$. For a commutative ring $A, \mathrm{GL}_{1}(A)=(A, \cdot)^{*}$. These remarks also apply to groups, monoids and rings that are not necessarily commutative.

Remark 2.5 For a $\log \operatorname{ring}(A, M, \alpha)$ we can factor the inclusion $\iota$ as

$$
\mathrm{GL}_{1}(A) \rightarrow M \stackrel{\alpha}{\rightarrow}(A, \cdot),
$$

by inverting the isomorphism $\tilde{\alpha}$. The log condition asserts that the part of $M$ that sits over the units of $A$ (via $\alpha$ ) is isomorphic to those units, and we view this as a normalization condition. The emphasis in a log structure is therefore on the part of $M$ that maps to the nonunits of $A$.

Definition 2.6 Let $(A, M, \alpha)$ be a pre-log ring. The associated log ring $\left(A, M^{a}, \alpha^{a}\right)$ consists of $A$ with the $\log$ structure $(M, \alpha)^{a}=\left(M^{a}, \alpha^{a}\right)$, where $M^{a}$ is defined by the upper left hand pushout square in the following diagram

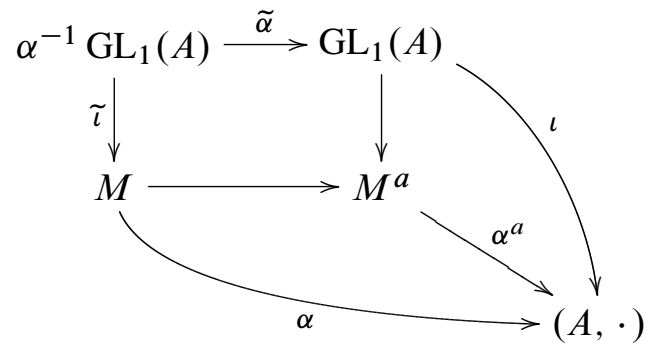

of commutative monoids, and $\alpha^{a}: M^{a} \rightarrow(A, \cdot)$ is the canonical homomorphism induced by $\alpha: M \rightarrow(A, \cdot)$ and $\iota: \mathrm{GL}_{1}(A) \rightarrow(A, \cdot)$. The next remark shows that $\left(A, M^{a}, \alpha^{a}\right)$ is indeed a log ring. A homomorphism $\left(f, f^{b}\right)$ of pre-log rings induces a homomorphism $\left(f, f^{\mathrm{b} a}\right):\left(A, M^{a}, \alpha^{a}\right) \rightarrow\left(B, N^{a}, \beta^{a}\right)$ of associated log rings. We obtain a logification functor $(-)^{a}: \mathcal{P}$ re $\mathcal{L} o g \rightarrow \mathcal{L}$ og .

Remark 2.7 Since $\mathrm{GL}_{1}(A)$ is an abelian group, the pushout

$$
M^{a}=M \oplus_{\alpha^{-1} \mathrm{GL}_{1}(A)} \mathrm{GL}_{1}(A)
$$


can be described as the balanced product $\left(M \times \mathrm{GL}_{1}(A)\right) / \sim$, where $(m, g) \sim\left(m^{\prime}, g^{\prime}\right)$ if and only if $m \cdot \tilde{\imath}\left(h_{1}\right)=m^{\prime} \cdot \widetilde{l}\left(h_{2}\right)$ and $\tilde{\alpha}\left(h_{2}\right) \cdot g=\widetilde{\alpha}\left(h_{1}\right) \cdot g^{\prime}$, for some $h_{1}, h_{2} \in$ $\alpha^{-1} \mathrm{GL}_{1}(A)$. See Kato [35, page 193]. We write $[m, g]$ for the equivalence class of $(m, g)$. The homomorphism $\alpha^{a}$ takes $[m, g] \in M^{a}$ to $\alpha(m) \cdot \iota(g) \in(A, \cdot)$, so $\alpha^{a}([m, g]) \in \mathrm{GL}_{1}(A)$ if and only if $\alpha(m) \in \mathrm{GL}_{1}(A)$, hence $[m, g]$ has a unique representative of the form $(1, h)$ with $h=\alpha(m)^{-1} g \in \mathrm{GL}_{1}(A)$, and $\left(M^{a}, \alpha^{a}\right)$ is really a $\log$ structure on $A$.

Lemma 2.8 The logification functor $(-)^{a}: \operatorname{Pr} \operatorname{L} \log \rightarrow \mathcal{L}$ og is left adjoint to the forgetful functor $\log \rightarrow \operatorname{Pre} \mathcal{L} o g$.

Definition 2.9 The trivial pre-log structure on $A$ is given by the trivial monoid $M=$ $\{1\}$ and the unique monoid homomorphism $\alpha:\{1\} \rightarrow(A, \cdot)$. The trivial log structure on $A$ is the associated $\log$ structure, with $M=\mathrm{GL}_{1}(A)$ and $\alpha=\imath: \mathrm{GL}_{1}(A) \rightarrow(A, \cdot)$.

Lemma 2.10 The functor $(-)^{\text {triv }}: \mathcal{C} \mathcal{R}$ ing $\rightarrow \mathcal{P r}$ e $\mathcal{L}$ og taking $A$ to the trivial pre-log ring $(A,\{1\})$ is left adjoint to the forgetful functor $\operatorname{Pr} e \mathcal{L} o g \rightarrow \mathcal{C} \mathcal{R} i n g$. Hence the functor $(-)^{\text {triv, } a}: \mathcal{C} \mathcal{R} i n g \rightarrow \mathcal{L} \log$ taking $A$ to the trivial $\log \operatorname{ring}\left(A, \mathrm{GL}_{1}(A)\right)$ is left adjoint to the forgetful functor $\mathcal{L} o g \rightarrow \mathcal{C} \mathcal{R} i n g$.

Remark 2.11 In algebro-geometric language, we can think of the opposite category $\log ^{\text {op }}$ as the category of affine log schemes, with a forgetful functor to the category $\mathcal{A} f f=\mathcal{C} \mathcal{R} i n g^{\text {op }}$ of affine schemes. The trivial log structure defines a right adjoint to the forgetful functor, embedding affine schemes into affine log schemes.

Definition 2.12 Let $M$ be a commutative monoid and $\mathbb{Z}[M]$ its monoid ring. The canonical pre-log structure on $\mathbb{Z}[M]$ is the pair $(M, \zeta)$, where $\zeta: M \rightarrow(\mathbb{Z}[M], \cdot)$ takes $m \in M$ to $1 \cdot m \in \mathbb{Z}[M]$. The canonical log structure on $\mathbb{Z}[M]$ is the associated $\log$ structure $(M, \zeta)^{a}$.

Lemma 2.13 The functor $(-)^{\text {can }}: \mathcal{C M}$ on $\rightarrow \mathcal{P r e} \mathcal{L}$ og taking $M$ to the canonical pre$\log$ ring $(\mathbb{Z}[M], M)$ is left adjoint to the forgetful functor Pre $\mathcal{L}$ og $\rightarrow \mathcal{C M}$ on. Hence the functor $(-)^{\text {can }, a}: \mathcal{C M}$ on $\rightarrow \mathcal{L} \log$ taking $M$ to the canonical log ring $\left(\mathbb{Z}[M], M^{a}\right)$ is left adjoint to the forgetful functor $\mathcal{L} o g \rightarrow \mathcal{C M}$ on .

Remark 2.14 We can summarize these adjunctions in the following diagram, where the unlabeled arrows denote forgetful functors:

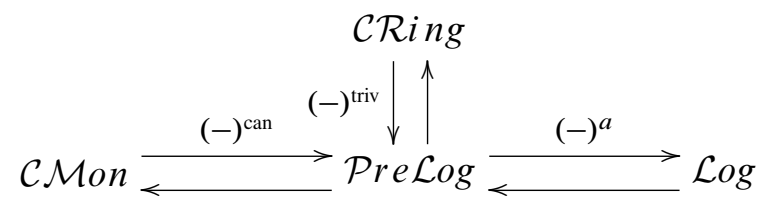


Any pre-log ring $(A, M)$ is the pushout

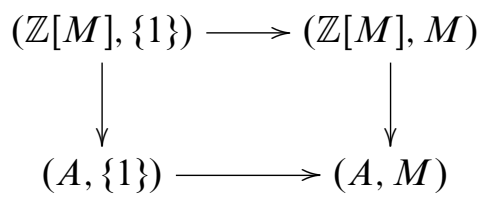

of a diagram of trivial and canonical pre-log rings. In this sense, the trivial and the canonical pre-log rings generate all pre-log rings.

Definition 2.15 For a pre-log ring $(A, M)$, the trivial locus is the pre-log ring $\left(A\left[M^{-1}\right], M^{\mathrm{gp}}\right)$ where

$$
A\left[M^{-1}\right]=A \otimes_{\mathbb{Z}[M]} \mathbb{Z}\left[M^{\mathrm{gp}}\right] .
$$

There is a canonical homomorphism $(A, M) \rightarrow\left(A\left[M^{-1}\right], M^{\mathrm{gp}}\right)$, and the associated $\log$ structure $\left(A\left[M^{-1}\right], M^{\mathrm{gp}}\right)^{a}$ equals the trivial one. For log rings $(A, M)$ the functor $(A, M) \mapsto A\left[M^{-1}\right]$ is left adjoint to $(-)^{\text {triv, } a}$, which therefore has both a left and a right adjoint.

Example 2.16 (This example is prominent in Lars Hesselholt and Ib Madsen's work [29].) Let $A$ be a discrete valuation ring, with uniformizer $\pi$. Let $M=\langle\pi\rangle=$ $\left\{\pi^{j} \mid j \geq 0\right\}$ be the free commutative monoid generated by $\pi$, and let $\alpha: M \rightarrow(A, \cdot)$ be the inclusion. Then $(A, M)=(A,\langle\pi\rangle)$ is a pre-log ring. The associated log ring $\left(A, M^{a}\right)$ has

$$
M^{a}=A \backslash\{0\} \cong\langle\pi\rangle \times \mathrm{GL}_{1}(A)
$$

equal to the multiplicative monoid of nonzero elements in $A$, and $\alpha^{a}: M \rightarrow(A, \cdot)$ equals the inclusion. Letting $K=A\left[\pi^{-1}\right]$ be the fraction field of $A$, we note that $M^{a}=A \cap \mathrm{GL}_{1}(K) \subset \mathrm{GL}_{1}(K)$. The trivial locus of $(A,\langle\pi\rangle)$ is $\left(K,\left\langle\pi, \pi^{-1}\right\rangle\right)$. A concrete example of interest to us is the case when $A=\mathbb{Z}_{p}$ the ring of $p$-adic integers, $\pi=p$ and $K=\mathbb{Q}_{p}$ is the $p$-adic field. See Serre [75, Section I.1] for other examples.

Remark 2.17 When we embed commutative rings into log rings (using the trivial log structures), the localization homomorphism $f: A \rightarrow K$ maps to the homomorphism $\left(f, f^{b}\right):\left(A, \mathrm{GL}_{1}(A)\right) \rightarrow\left(K, \mathrm{GL}_{1}(K)\right)$. One essential feature of the category $\mathcal{L} o g$ is that the latter homomorphism factors as the composite

$$
\left(A, \mathrm{GL}_{1}(A)\right) \rightarrow\left(A, M^{a}\right) \rightarrow\left(K, \mathrm{GL}_{1}(K)\right),
$$

where the middle term is a log ring with a nontrivial log structure. In geometric terms, the open inclusion $j: U=\operatorname{Spec}(K) \rightarrow \operatorname{Spec}(A)=X$ of the generic point does not 
factor in any nontrivial way in $\mathcal{A} f f$, but when viewed as a map of affine log schemes it factors as

$$
U \rightarrow \Lambda=\operatorname{Spec}\left(A, M^{a}\right) \rightarrow X,
$$

where $\Lambda=\operatorname{Spec}\left(A, M^{a}\right)$ is properly a $\log$ scheme. Heuristically, $\Lambda$ is a kind of compactification of $U$, and $\Lambda \rightarrow X$ specifies a less dramatic localization of $X$ (in $\log$ schemes) than the open inclusion $U \rightarrow X$ (in schemes). See Kato-Nakayama [36, (1.2)] and Illusie [34, Section 5.5] for a more precise interpretation (in a complex analytic setting) of the log scheme as a compactification of the trivial locus.

Remark 2.18 (I learned of this point of view from Clark Barwick.) Following Martin Olsson [54, Theorem 1.1], one can embed the category of log schemes into the 2category of algebraic stacks, by taking a log scheme $\Lambda$ to a suitable moduli category $\log (\Lambda)=\operatorname{str} \log / \Lambda$ of $\log$ schemes over $\Lambda$ and "strict" morphisms between these (see Definition 2.22). To be precise, Kato and Olsson only work with "fine" log structures (see Definition 3.1). This means that Spec of a fine log ring acquires a geometric meaning in the context of algebraic stacks, and, in particular, that the factorization $U \rightarrow \Lambda \rightarrow X$ of $j$ can be viewed as taking place in that context. Geometric notions like flat, smooth, unramified, étale and fppf (= faithfully flat and finitely presented) morphisms of log rings, or log schemes, then become special cases of the same notions for algebraic stacks.

Definition 2.19 Let $f: A \rightarrow B$ be a ring homomorphism and let $(M, \alpha)$ be a pre-log structure on $A$. The inverse image log structure

$$
\left(f^{*} M, f^{*} \alpha\right)=(M,(f, \cdot) \circ \alpha)^{a}
$$

on $B$ is the log structure associated to the pre-log structure given by the composite monoid homomorphism

$$
M \stackrel{\alpha}{\rightarrow}(A, \cdot) \stackrel{(f, \cdot)}{\longrightarrow}(B, \cdot) .
$$

There is a canonical homomorphism $\left(f, f^{b}\right):(A, M) \rightarrow\left(B, f^{*} M\right)$ of (pre-)log rings.

Remark 2.20 Note that $(M,(f, \cdot) \circ \alpha)$ is usually not a $\log$ structure on $B$, before logification, even if $(M, \alpha)$ is a $\log$ structure on $A$. The variance of the terminology and notation (inverse image, $f^{*}$ ) is compatible with that used in algebraic geometry, when $f$ is viewed as a map $\operatorname{Spec}(B) \rightarrow \operatorname{Spec}(A)$ in $\mathcal{A} f f$ and the log structure is a sheaf over $\operatorname{Spec}(A)$. The variance is perhaps counterintuitive in the context of commutative rings, but switching the roles of $f^{*}$ and $f_{*}$ (defined below) would make the comparison with the algebro-geometric literature prohibitively confusing. 
Lemma 2.21 The $\log$ homomorphisms $(A, M) \rightarrow(B, N)$ covering a fixed ring homomorphism $f: A \rightarrow B$ are in natural bijection with the log homomorphisms $\left(B, f^{*} M\right) \rightarrow(B, N)$ covering the identity $\mathrm{id}_{B}$ on $B$.

Definition 2.22 A homomorphism $\left(f, f^{b}\right):(A, M) \rightarrow(B, N)$ of log rings is strict if the corresponding monoid homomorphism $f^{*} M \rightarrow N$ is an isomorphism. We write str $\mathcal{L} \log \subset \mathcal{L} \operatorname{og}$ for the subcategory of strict homomorphisms.

Definition 2.23 Let $f: A \rightarrow B$ be a ring homomorphism and let $(N, \beta)$ be a pre-log structure on $B$. The direct image pre-log structure $\left(f_{*} N, f_{*} \beta\right)$ on $A$ is defined by the pullback square

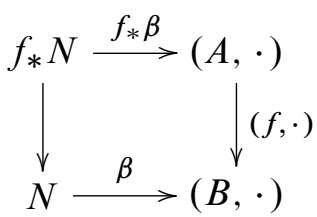

of commutative monoids. When $(N, \beta)$ is a $\log$ structure on $B,\left(f_{*} N, f_{*} \beta\right)$ will also be a $\log$ structure, the direct image log structure on $A$. There is a canonical homomorphism $\left(f, f^{\mathrm{b}}\right):\left(A, f_{*} N\right) \rightarrow(B, N)$ of (pre-)log rings.

Lemma 2.24 The $\log$ homomorphisms $(A, M) \rightarrow(B, N)$ covering a fixed ring homomorphism $f: A \rightarrow B$ are in natural bijection with the log homomorphisms $(A, M) \rightarrow\left(A, f_{*} N\right)$ covering the identity $\operatorname{id}_{A}$ on $A$.

Remark 2.25 For a discrete valuation ring $A$, the $\log$ structure $M^{a}=A \backslash\{0\}=$ $A \cap \mathrm{GL}_{1}(K)$ from Example 2.16 is the same as the direct image $f_{*} \mathrm{GL}_{1}(K)$ of the trivial $\log$ structure on $K$, along the homomorphism $f: A \rightarrow K$. Hence the direct image construction naturally produces the factorization in log schemes from Remark 2.17. More generally, for ring homomorphisms $f: A \rightarrow B$ the direct image $M=f_{*} \mathrm{GL}_{1}(B)$ of the trivial $\log$ structure on $B$ provides a $\log$ ring $(A, M)$ that may serve as an approximation to $B$. In the topological setting of the following sections, this provides a useful $\log$ structure on $A$ in the cases where $B$ exists, but it will be less useful when the desired $B$ does not exist and we are trying to construct $(A, M)$ as a replacement for the nonexistent $B$.

\section{Replete logarithmic structures}

Definition 3.1 We now review some desirable properties of log rings and log schemes, with the aim to motivate the introduction in Definitions 3.6 and 3.12 of another such property. See Kato [35, Section 2] and Nakayama [52, Section 1]. 
In the affine cases that we consider, every $\log$ structure $(M, \alpha)$ on a commutative ring $A$ is quasi-coherent. It is coherent if $M$ is finitely generated as a commutative monoid. A commutative monoid $M$ is integral if the canonical homomorphism $\gamma: M \rightarrow M^{\text {gp }}$ to its group completion is injective. It is fine if it is finitely generated and integral. An integral $M$ is saturated if the only $a \in M^{\mathrm{gp}}$ with $a^{n} \in M$ for some $n \in \mathbb{N}$ are the $a \in M$. It is $f_{s}$ if it is fine and saturated. We say that a pre-log structure $(M, \alpha)$, or a pre-log ring $(A, M)$, is integral, fine, saturated or fs, respectively, if the commutative monoid $M$ has the corresponding property. A log ring is said to have one of these properties if it is isomorphic to the logification of a pre-log ring with the given property. Let $M^{\text {int }}=\gamma(M) \subset M^{\text {gp }}$ be the image of $M$, and let $M^{\text {sat }} \subset M^{\text {gp }}$ consist of all $a \in M^{\text {gp }}$ with $a^{n} \in M$ for some $n \in \mathbb{N}$. These constructions preserve the subcategories of finitely generated commutative monoids, and restrict to define left adjoints $(-)^{\text {fine }}=$ $\left.(-)^{\text {int }}\right|_{\mathcal{C M} n^{\mathrm{fg}}}$ and $(-)^{\mathrm{fs}}=\left.(-)^{\mathrm{sat}}\right|_{\mathcal{C} \mathcal{M} n^{\mathrm{fine}}}$ to the forgetful functors

$$
\mathcal{C M o n}{ }^{\mathrm{fs}} \stackrel{(-)^{\mathrm{fs}}}{\longrightarrow} \mathcal{C M} \operatorname{Mn}^{\mathrm{fine}} \stackrel{(-)^{\mathrm{fine}}}{\longrightarrow} \mathcal{C M o n}{ }^{\mathrm{fg}}
$$

between fs, fine and finitely generated commutative monoids, respectively. The category $\mathcal{C} \mathcal{M o n}{ }^{\mathrm{fg}}$ has all finite colimits. The left adjoint functors $(-)^{\text {fine }}$ and $(-)^{\mathrm{fs}}$ can therefore be used to create finite colimits in the subcategories $\mathcal{C} \mathcal{M}$ on ${ }^{\text {fine }}$ and $\mathcal{C} \mathcal{M}$ on ${ }^{\mathrm{fs}}$.

Finite colimits in the category of fine pre-log rings are constructed by first forming the finite colimit in coherent pre-log rings. The result, $(A, M)$ say, is then replaced by the fine base change $(A, M)^{\text {fine }}=\left(A \otimes_{\mathbb{Z}[M]} \mathbb{Z}\left[M^{\text {fine }}\right], M^{\text {fine }}\right)$. Similarly, finite colimits in the category of fs pre-log rings are constructed by first forming $(B, N)=(A, M)^{\text {fine }}$ as above, and then replacing it by the fs base change $(B, N)^{\mathrm{fs}}=\left(B \otimes_{\mathbb{Z}[N]} \mathbb{Z}\left[N^{\mathrm{fs}}\right], N^{\mathrm{fs}}\right)$. The corresponding construction in fine (resp. fs) log rings is obtained by applying logification at the end.

Remark 3.2 In the study of smoothness properties and deformation theory for log rings or log schemes (see Kato [35] and Olsson [55]), it is common to work with thickenings $\left(g, g^{b}\right):(A, M) \rightarrow(R, P)$ that are strict morphisms to a fixed base log ring $(R, P)$, ie, such that $g^{*} M \cong P$, where $A / J \cong R$ for some square zero (or nil) ideal $J$. The strictness hypothesis leads to the key property that the diagram

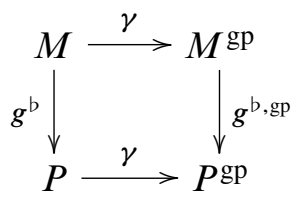

is a pullback square of commutative monoids. In other words, $g^{\mathrm{b}}: M \rightarrow P$ is "exact" (see Definition 3.3). 
Furthermore, it is common to work within the subcategory $\mathcal{L} o g^{\text {fine }}$ of fine log rings (resp. fine log schemes). This ensures that the canonical log rings $M^{\text {can, } a}=\left(\mathbb{Z}[M], M^{a}\right)$ have underlying rings of finite type, as is convenient in algebraic geometry. It also ensures that the natural homomorphism

$$
\gamma^{\mathrm{can}, a}:\left(\mathbb{Z}[M], M^{a}\right) \rightarrow\left(\mathbb{Z}\left[M^{\mathrm{gp}}\right], M^{\mathrm{gp}, a}\right)=\mathbb{Z}\left[M^{\mathrm{gp}}\right]^{\text {triv }}
$$

describes an embedding of the diagonalizable (affine, commutative) group scheme $D\left(M^{\mathrm{gp}}\right)=\operatorname{Spec}\left(\mathbb{Z}\left[M^{\mathrm{gp}}\right]\right.$ ) (a product of $\mathbb{G}_{m}$ 's and $\mu_{n}$ 's) in the affine log scheme $\operatorname{Spec}\left(\mathbb{Z}[M], M^{a}\right)$ that is "dense" in a suitable sense, rather than one that properly factors through a closed log subscheme $\operatorname{Spec}\left(\mathbb{Z}\left[M^{\text {int }}\right], M^{\text {int }, a}\right)$. This leads to the close connection between logarithmic geometry and the theory of toroidal embeddings.

Definition 3.3 A monoid homomorphism $\epsilon: M \rightarrow P$ is exact if the diagram

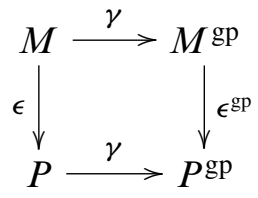

is a pullback square.

Remark 3.4 In the study of log étale cohomology, Kummer étale $K$-theory and log $K$-theory (see Nakayama [52], Hagihara [26] and Nizioł [53]), it is common to restrict further to the subcategory $\mathcal{L}_{\log }$ fs of fs log rings. To illustrate why, we focus on the case of a $\log G-$ Galois extension $\left(f, f^{b}\right):(A, M) \rightarrow(B, N)$, where $G=\operatorname{Spec}(H)$ is a finite étale group scheme over $\operatorname{Spec}(A)$ that acts on $\operatorname{Spec}(B, N)$ over $\operatorname{Spec}(A, M)$. For $\left(f, f^{b}\right)$ to be $\log G-$ Galois, we require that the canonical map

$$
h:(B, N) \otimes_{(A, M)}^{\mathrm{fs}}(B, N) \rightarrow H \otimes_{A}(B, N)
$$

is an isomorphism, plus that $f: A \rightarrow B$ is faithfully flat. The interpretation of the $\log$ ring tensor product (= pushout) in the source of $h$ is now dependent on the categorical context. In fs log rings, the underlying monoid will be the integral saturation $\left(N \oplus_{M} N\right)^{\text {fs }}$ of the pushout $N \oplus_{M} N$ formed in commutative monoids. Similarly, the underlying commutative ring will be the base change of $B \otimes_{A} B$ along $\mathbb{Z}\left[N \oplus_{M} N\right] \rightarrow$ $\mathbb{Z}\left[\left(N \oplus_{M} N\right)^{\mathrm{fs}}\right]$. This saturation significantly extends the range of examples of log Galois extensions.

For example, suppose that $f^{b}: M \rightarrow N$ is an injective homomorphism of fs commutative monoids, and that there is a natural number $k$ such that $f^{b}(M)$ contains $N^{k}=\left\{n^{k} \mid n \in N\right\} \subset N$. Such homomorphisms are called Kummer homomorphisms. The cokernel $C$ of $f^{\mathrm{b}, \mathrm{gp}}: M^{\mathrm{gp}} \rightarrow N^{\mathrm{gp}}$ is then a finite group of exponent $k$. 
Let $\bar{\gamma}: N \rightarrow C$ be the canonical monoid homomorphism. Let $A=\mathbb{Z}[1 / k][M]$, $B=\mathbb{Z}[1 / k][N]$ and $H=A[C]$. Then $H$ is an étale (bi-)commutative Hopf algebra over $A$, which coacts on $(B, N)$ by the $\log$ ring homomorphism

$$
(B, N) \rightarrow H \otimes_{A}(B, N)
$$

under $(A, M)$ induced by the monoid homomorphism $N \rightarrow C \times N$ that takes $n$ to $(\bar{\gamma}(n), n)$. Kato showed (see Illusie [34, Proposition 3.2]) that $\left(f, f^{b}\right):\left(A, M^{a}\right) \rightarrow$ $\left(B, N^{a}\right)$ is a (Kummer étale) $G$-Galois extension, with $G=\operatorname{Spec}(H)=D(C)_{\operatorname{Spec}(A)}$. The main point is that the monoid homomorphism $N \oplus_{M} N \rightarrow C \times N$ that takes the class of $n_{1} \oplus n_{2}$ to $\left(\bar{\gamma}\left(n_{1}\right), n_{1} n_{2}\right)$ is usually not surjective, but the induced map from its integral saturation

$$
\left(N \oplus_{M} N\right)^{\mathrm{fs}} \stackrel{\cong}{\rightarrow} C \times N
$$

is always an isomorphism. As a simple example, the reader might wish to consider the case $M=\langle y\rangle$ and $N=\langle x\rangle$, with $f^{b}(y)=x^{k}$ for some $k \geq 2$. This example makes it clear that it is the Kummer condition on $f^{b}: M \rightarrow N$ that makes all the elements in $C \times N$ have a positive power that is in the image from $N \oplus_{M} N$, so that saturation suffices to extend $N \oplus_{M} N$ to cover all of $C \times N$.

In the setting of a Kummer homomorphism $f^{\text {b }}: M \rightarrow N$, the integral saturation $\left(N \oplus_{M} N\right)^{\mathrm{fs}}$ has a different characterization. We view $N \oplus_{M} N$ as a commutative monoid over $N$, via $\epsilon: N \oplus_{M} N \rightarrow N$ taking the class of $n_{1} \oplus n_{2}$ to $n_{1} n_{2}$, and note that the factorization of $\gamma: N \oplus_{M} N \rightarrow\left(N \oplus_{M} N\right)^{\mathrm{gp}}$ through $\left(N \oplus_{M} N\right)^{\mathrm{fs}}$ has the property that the right hand square in the commutative diagram

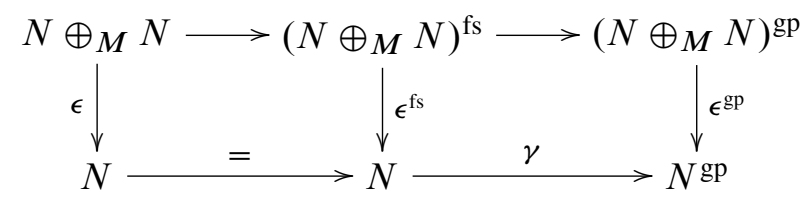

is a pullback square of commutative monoids. This is clear, because the preimage $\left(\epsilon^{\mathrm{fs}}\right)^{-1}(n)$ of $n \in N$ is identified with $C \times\{n\}$ under the isomorphism $\left(N \oplus_{M} N\right)^{\mathrm{fs}} \cong$ $C \times N$, and the preimage $\left(\epsilon^{\mathrm{gp}}\right)^{-1}(\gamma(n))$ is identified with $C \oplus \gamma(n)$ under the splitting $\left(N \oplus_{M} N\right)^{\mathrm{gp}} \cong N^{\mathrm{gp}} \oplus_{M^{\mathrm{gp}}} N^{\mathrm{gp}} \cong C \oplus N^{\mathrm{gp}}$ that comes from the inclusion of $N^{\mathrm{gp}}$ in the second summand of $N^{\mathrm{gp}} \oplus_{M^{\mathrm{gp}}} N^{\mathrm{gp}}$. The induced map $\left(N \oplus_{M} N\right)^{\mathrm{fs}} \rightarrow\left(N \oplus_{M} N\right)^{\mathrm{gp}}$ identifies $C \times\{n\}$ with $C \oplus \gamma(n)$, for each $n \in N$.

It also follows that $\left(N \oplus_{M} N\right)^{\mathrm{gp}}$ is the group completion of $\left(N \oplus_{M} N\right)^{\mathrm{fs}}$, so $\epsilon^{\mathrm{fs}}:\left(N \oplus_{M} N\right)^{\mathrm{fs}} \rightarrow N$ is exact.

Remark 3.5 When generalizing the algebraic theory of log rings to the topological setting, it is not so clear what should replace the properties of being integral and saturated. 
It also appears restrictive to only work with finitely generated commutative monoids. Given the observations in Remarks 3.2 and 3.4, we are therefore led to focus on the exact homomorphisms $\epsilon: M \rightarrow P$. We view exactness as a condition on a commutative monoid $M$ relative to a base commutative monoid $P$. In the applications we have in mind, such as abelian group objects in $\mathcal{C}$ Mon $/ P$, or coproducts of multiple copies of $P$, the structural map $\epsilon: M \rightarrow P$ will have a (sometimes preferred) section $\eta: P \rightarrow M$. However, the following definition has a topologically meaningful generalization as soon as $\epsilon^{\mathrm{gp}}: M^{\mathrm{gp}} \rightarrow P^{\mathrm{gp}}$ is surjective (see Proposition 8.3 ), so that is what we will assume.

Definition 3.6 Let $\epsilon: M \rightarrow P$ be a homomorphism of commutative monoids, viewed as an object in the category $\mathcal{C M o n} / P$ of commutative monoids over $P$. We say that $\epsilon: M \rightarrow P$ is virtually surjective if the induced homomorphism $\epsilon^{\mathrm{gp}}: M^{\mathrm{gp}} \rightarrow P^{\mathrm{gp}}$ of abelian groups is surjective. Let $(\mathcal{C M o n} / P)^{\text {vsur }} \subset \mathcal{C M o n} / P$ be the full subcategory of virtually surjective $M$ over $P$. We say that a virtually surjective $M$ over $P$ is replete if it is also exact, ie, if the commutative diagram

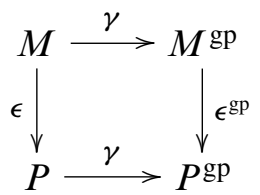

is a pullback square. Let $(\mathcal{C M o n} / P)^{\text {rep }} \subset(\mathcal{C M o n} / P)^{\text {vsur }}$ be the full subcategory of replete commutative monoids $M$ over $P$

For a general virtually surjective $\epsilon: M \rightarrow P$, let the repletion of $M$ over $P$ be the pullback

$$
M^{\mathrm{rep}}=P \times_{P \text { gp }} M^{\mathrm{gp}}
$$

in the diagram above, with the canonical structure map $\epsilon^{\text {rep }}: M^{\text {rep }} \rightarrow P$. The following diagram commutes, where the right hand square is a pullback by construction:

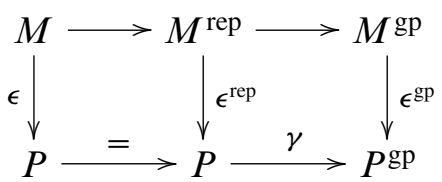

We call $M \rightarrow M^{\text {rep }}$ the repletion map, and show in Lemma 3.8 below that $M^{\text {rep }}$ is replete.

Remark 3.7 For $M$ to be replete over $P$ is equivalent to $\epsilon: M \rightarrow P$ being an exact surjection. We view repleteness as a property of virtually surjective $M$ over $P$, since 
it is for such $M$ that we will prove that repletion is an idempotent functor. We also prefer to distinguish between "replete" and "exact", because exactness is usually taken to be a property of homomorphisms between integral commutative monoids.

Lemma 3.8 For virtually surjective $\epsilon: M \rightarrow P$, the homomorphisms $M \rightarrow M^{\text {rep }} \rightarrow$ $M^{\mathrm{gp}}$ induce isomorphisms

$$
M^{\mathrm{gp}} \cong\left(M^{\mathrm{rep}}\right)^{\mathrm{gp}} \cong\left(M^{\mathrm{gp}}\right)^{\mathrm{gp}}
$$

upon group completion. Hence $M^{\text {rep }}$ is replete over $P$.

Proof It is easy to see that $\left(M^{\text {rep }}\right)^{\mathrm{gp}} \rightarrow\left(M^{\mathrm{gp}}\right)^{\mathrm{gp}}$ is surjective, since every element of $\left(M^{\mathrm{gp}}\right)^{\mathrm{gp}} \cong M^{\mathrm{gp}}$ is the difference of two elements coming from $M$. To prove injectivity, consider a formal difference $\left(p_{1}, \bar{m}_{1}\right) \ominus\left(p_{2}, \bar{m}_{2}\right)$ in $\left(M^{\text {rep }}\right)^{\mathrm{gp}}$, with $p_{i} \in P$, $\bar{m}_{i} \in M^{\mathrm{gp}}$ and $\gamma\left(p_{i}\right)=\epsilon^{\mathrm{gp}}\left(\bar{m}_{i}\right)$ for $i=1,2$, and assume that its image $\bar{m}_{1} \ominus \bar{m}_{2}$ is zero in $\left(M^{\mathrm{gp}}\right)^{\mathrm{gp}}$. Then $\bar{m}_{1}=\bar{m}_{2}$, so $\gamma\left(p_{1}\right)=\gamma\left(p_{2}\right)$, and there exists a $k \in P$ with $p_{1}+k=p_{2}+k$. Using the surjectivity of $\epsilon^{\mathrm{gp}}$, we can chose an $\bar{\ell} \in M^{\mathrm{gp}}$ with $\epsilon^{\mathrm{gp}}(\bar{\ell})=\gamma(k)$. Then $\left(p_{1}, \bar{m}_{1}\right)+(k, \bar{\ell})=\left(p_{2}, \bar{m}_{2}\right)+(k, \bar{\ell})$, so $\left(p_{1}, \bar{m}_{1}\right) \ominus\left(p_{2}, \bar{m}_{2}\right)$ is zero in $\left(M^{\mathrm{rep}}\right)^{\mathrm{gp}}$.

To see that $M^{\text {rep }}$ is replete, note that it is isomorphic to the pullback of $\gamma: P \rightarrow P^{\mathrm{gp}}$ and $\left(M^{\text {rep }}\right)^{\mathrm{gp}} \rightarrow P^{\mathrm{gp}}$, since the latter map is isomorphic to $\epsilon^{\mathrm{gp}}: M^{\mathrm{gp}} \rightarrow P^{\mathrm{gp}}$.

Lemma 3.9 The functor $(-)^{\mathrm{rep}}:(\mathcal{C} \text { Mon } / P)^{\mathrm{vsur}} \rightarrow(\mathcal{C M o n} / P)^{\text {rep }}$ is left adjoint to the forgetful functor. Colimits of nonempty diagrams in $(\mathcal{C M o n} / P)^{\mathrm{vsur}}$ exist and are created in $\mathcal{C} M o n / P$. Colimits of nonempty diagrams also exist in $(\mathcal{C M o n} / P)^{\text {rep }}$, and are constructed by first forming the colimit in $(\mathcal{C M o n} / P)^{\text {vsur }}$ and then applying $(-)^{\text {rep }}$.

Definition 3.10 Let $P / \mathcal{C M o n} / P$ be the category of commutative monoids under and over $P$, ie, triples $(M, \eta, \epsilon)$ where $\eta: P \rightarrow M$ and $\epsilon: M \rightarrow P$ are commutative monoid homomorphisms with $\epsilon \circ \eta=$ id. The forgetful functor $P / \mathcal{C M o n} / P \rightarrow \mathcal{C M}$ on $/ P$ factors through the full subcategory $(\mathcal{C M o n} / P)^{\text {vsur }}$. We say that $(M, \eta, \epsilon)$ is replete over $P$ if the underlying virtually surjective $\epsilon: M \rightarrow P$ is replete.

Lemma 3.11 An object $(M, \eta, \epsilon)$ in $P / \mathcal{C M o n} / P$ is replete if and only if it is isomorphic to an object of the form $\left(P \times K, \eta_{0}, \epsilon_{0}\right)$, where $K$ is an abelian group with unit element $e, \eta_{0}(p)=(p, e)$ and $\epsilon_{0}(p, k)=p$. If so, there are isomorphisms $K \cong \operatorname{ker}\left(\epsilon^{\mathrm{gp}}\right) \cong \operatorname{cok}\left(\eta^{\mathrm{gp}}\right)$, and the isomorphism $M \cong P \times K$ takes $m$ to $(\epsilon(m), \bar{\gamma}(m))$, where $\bar{\gamma}: M \rightarrow M^{\mathrm{gp}} \rightarrow K$ is the canonical map. In particular, $\left(M^{\mathrm{rep}}, \eta^{\text {rep }}, \epsilon^{\text {rep }}\right)$ is always replete. 
Proof In this split case, $M^{\mathrm{gp}}$ is isomorphic to $P^{\mathrm{gp}} \times K$, so to be replete $M$ must be isomorphic to $P \times K$. Conversely, if $M$ is isomorphic to $P \times K$, then $M^{\text {gp }}$ is isomorphic to $P^{\mathrm{gp}} \times K$, and $M$ will be replete.

Definition 3.12 Let $(R, P)$ be a base pre-log ring. A pre-log ring $(A, M)$ over $(R, P)$ is virtually surjective if the underlying commutative monoid $M$ over $P$ is virtually surjective. It is a replete pre-log ring if the underlying commutative monoid $M$ over $P$ is replete. It is a replete log ring if $(A, M)$ is also a log ring. By Proposition 3.14, the logification of a replete pre-log ring over an integral log ring is a replete log ring. Let $(\mathcal{P r e} \log /(R, P))^{\text {vsur }}$ be the full subcategory of $\operatorname{Pre} \mathcal{L} o g /(R, P)$ generated by the pre-log rings that are virtually surjective over $(R, P)$, and let $(\mathcal{P r e} \mathcal{L} o g /(R, P))^{\text {rep }}$ be the full subcategory generated by the replete pre-log rings. The forgetful functor $(R, P) / \operatorname{Pre} \mathcal{L} o g /(R, P) \rightarrow \operatorname{Pre} \mathcal{L} o g /(R, P)$ naturally factors through $(\mathcal{P r e} \log /(R, P))^{\text {vsur }}$. Let the repletion functor

$$
(-)^{\mathrm{rep}}:(\operatorname{Pre} \mathcal{L} \operatorname{og} /(R, P))^{\mathrm{vsur}} \rightarrow(\operatorname{Pr} e \mathcal{L} \operatorname{og} /(R, P))^{\mathrm{rep}}
$$

be the left adjoint to the forgetful functor, taking a virtually surjective pre-log ring $(A, M)$ over $(R, P)$ to the replete pre-log ring

$$
(A, M)^{\mathrm{rep}}=\left(A \otimes_{\mathbb{Z}[M]} \mathbb{Z}\left[M^{\mathrm{rep}}\right], M^{\mathrm{rep}}\right)
$$

over $(R, P)$. Colimits over nonempty diagrams exist in $(\mathcal{P r e} \mathcal{L} o g /(R, P))^{\text {vsur }}$, and are created in $\mathcal{P r e} \log /(R, P)$. Nonempty colimits in $(\mathcal{P r e} \mathcal{L} o g /(R, P))^{\text {rep }}$ are constructed by first forming the colimit in $(\mathcal{P r} e \mathcal{L} o g /(R, P))^{\text {vsur }}$, and then applying $(-)^{\text {rep }}$.

Lemma 3.13 Let $(A, M, \alpha)$ be a replete pre-log ring over a $\log$ ring $(R, P, \rho)$. Then $M^{*}=\alpha^{-1} \mathrm{GL}_{1}(A)$.

Proof Consider the following diagram

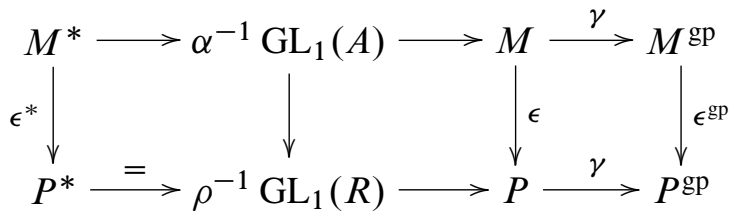

of commutative monoids. The left hand and middle horizontal maps are inclusions. By hypothesis the group homomorphism $\epsilon^{\mathrm{gp}}$ is surjective, with kernel $K$, say, the right hand square is a pullback, and the inclusion $P^{*} \rightarrow \rho^{-1} \mathrm{GL}_{1}(R)$ is the identity.

We first prove that $M^{*} \rightarrow P^{*}$ is surjective, with kernel $K$. If $p \in P^{*}$ with inverse $q$, we can find $m, n \in M$ with $\epsilon(m)=p$ and $\epsilon(n)=q$. Then $\epsilon(m n)=e$, so $m n=k$ 
lies in $\epsilon^{-1}(e) \cong K$. Now $K$ is a group, so we can form $n k^{-1} \in M$, which is inverse to $m$. Hence $m \in M^{*}$, and $m$ maps to $p$, so $M^{*} \rightarrow P^{*}$ is surjective. Its kernel is $M^{*} \cap \epsilon^{-1}(e)=\epsilon^{-1}(e) \cong K$, where the inclusion $\epsilon^{-1}(e) \subset M^{*}$ holds because $\epsilon^{-1}(e) \cong K$ is a group.

It follows that $M^{*}$ is the pullback of $M$ and $P^{*}$ over $P$. On the other hand, $\alpha^{-1} \mathrm{GL}_{1}(A)$ is contained in the pullback of $M$ and $\rho^{-1} \mathrm{GL}_{1}(R)$ over $P$, since $\mathrm{GL}_{1}(A) \subset(A, \cdot)$ maps to $\mathrm{GL}_{1}(R) \subset(R, \cdot)$. By assumption, $P^{*}=\rho^{-1} \mathrm{GL}_{1}(R)$, so $M^{*}=\alpha^{-1} \mathrm{GL}_{1}(A)$.

Proposition 3.14 Let $(A, M)$ be a replete pre-log ring over an integral log ring $(R, P)$. Then the associated $\log \operatorname{ring}\left(A, M^{a}\right)$ is a replete log ring over $(R, P)$.

Proof By assumption, $\gamma: P \rightarrow P^{\mathrm{gp}}$ is injective, so its pullback $\gamma: M \rightarrow M^{\mathrm{gp}}$ is also injective. Hence $(A, M)$ is integral, and $M^{*}$ acts freely on $M$ and $M^{\text {gp }}$.

By Lemma 3.13, $M^{a}$ is the pushout of $M$ and $\mathrm{GL}_{1}(A)$ along $M^{*}$, so $M^{a, \mathrm{gp}}$ is the pushout of $M^{\mathrm{gp}}$ and $\mathrm{GL}_{1}(A)$ along $M^{*}$.

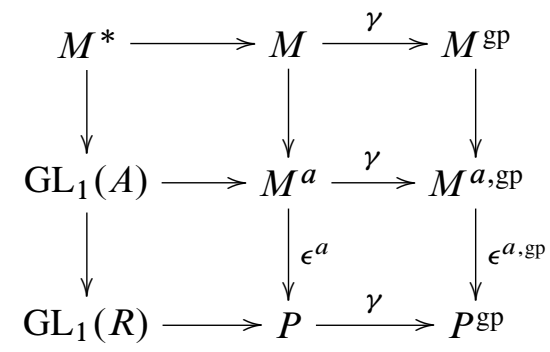

The composite

$$
\epsilon^{\mathrm{gp}}: M^{\mathrm{gp}} \rightarrow M^{a, \mathrm{gp}} \rightarrow P^{\mathrm{gp}}
$$

is surjective, hence $\epsilon^{a}: M^{a} \rightarrow P$ is virtually surjective. In the commutative diagram

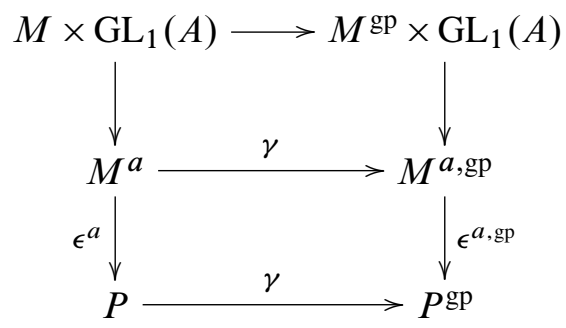

the outer rectangle is a pullback, and the middle row is obtained from the upper row by dividing out by a free $M^{*}$-action, hence the lower square is a pullback. This proves that $\left(A, M^{a}\right)$ is a replete log ring over $(R, P)$. 
Example 3.15 Let $(A, M)$ be a pre-log ring and $Y$ a nonempty set. The $Y$-fold replete tensor product $Y \otimes^{\text {rep }}(A, M)$ is the replete pre-log ring $\left(Y \otimes^{\text {rep }} A, \prod_{Y}^{\text {rep }} M\right)$ over $(A, M)$ given by the pushout

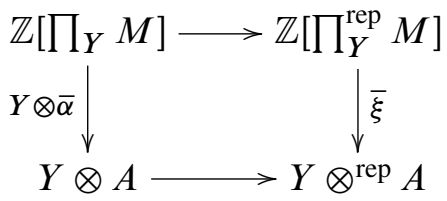

of commutative rings, and the pre-log structure

$$
\xi: \prod_{Y}^{\text {rep }} M \rightarrow\left(Y \otimes^{\text {rep }} A, \cdot\right)
$$

right adjoint to $\bar{\xi}$, where $Y \otimes M=\prod_{Y} M \rightarrow M$ is the cartesian product (= coproduct) in $\mathcal{C}$ Mon $/ M$ of $Y$ copies of id: $M \rightarrow M, \prod_{Y}^{\text {rep }} M=\left(\prod_{Y} M\right)^{\text {rep }} \cong M \times \prod_{X} M^{\text {gp }}$ is its repletion, where $X$ is the complement of one element in $Y$, and $Y \otimes A=\otimes_{Y} A$ is the tensor product (= coproduct) in $\mathcal{C}$ Ring of $Y$ copies of $A$.

Definition 3.16 Let $M$ be a commutative monoid, and let $S_{\bullet}^{1}=\Delta_{\bullet}^{1} / \partial \Delta_{\bullet}^{1}$ be the simplicial circle. The cyclic bar construction $B^{\mathrm{cy}} M=S_{\bullet}^{1} \otimes M$ (called the cyclic nerve $N^{\text {cy }} M$ in Waldhausen [79, Section 2.3]) is the simplicial commutative monoid given by the categorical tensor product

$$
S_{q}^{1} \otimes M=\prod_{S_{q}^{1}} M \cong M \times M \times \cdots \times M
$$

$((q+1)$ copies of $M)$ in simplicial degree $q$. We write a typical element of $B^{\text {cy }} M$ as $\left(m_{0}, m_{1}, \ldots, m_{q}\right)$.

There are natural structure maps $\eta: M \rightarrow B^{\text {cy }} M$ and $\epsilon: B^{\text {cy }} M \rightarrow M$ induced by the base point inclusion $* \rightarrow S_{\bullet}^{1}$ and the collapse map $S_{\bullet}^{1} \rightarrow *$. The map $\eta$ equals the inclusion of the zero-simplices in $B^{\mathrm{cy}} M$, while $\epsilon$ takes $\left(m_{0}, m_{1}, \ldots, m_{q}\right)$ to the product $m_{0} m_{1} \cdots m_{q}$. These maps make $B^{\text {cy }} M$ a simplicial object in $M / \mathcal{C}$ Mon $/ M$. There is a natural cyclic structure on $B^{\mathrm{cy}} M$, generated by the operator $t_{q}$ that takes $\left(m_{0}, m_{1}, \ldots, m_{q}\right)$ to $\left(m_{q}, m_{0}, \ldots, m_{q-1}\right)$. We give $M$ the constant cyclic structure, and then $\epsilon$ (but not $\eta$ ) is a cyclic morphism. There is a natural projection $\pi: B^{\text {cy }} M \rightarrow$ $B M$ to the ordinary bar construction on $M$, taking $\left(m_{0}, m_{1}, \ldots, m_{q}\right)$ to $\left[m_{1}|\cdots| m_{q}\right]$, forgetting the copy of $M$ that corresponds to the base point in $S_{\bullet}^{1}$. 
The replete bar construction $B^{\text {rep }} M=\left(B^{\text {cy }} M\right)^{\text {rep }}$ is the repletion of the cyclic bar construction, given by the lower right hand pullback square

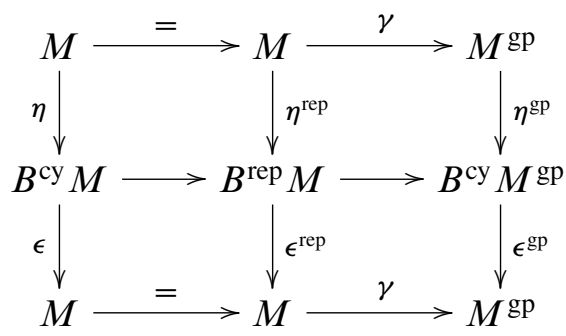

of simplicial commutative monoids. Here $\gamma$ and $\epsilon^{\mathrm{gp}}$ are cyclic maps, so the definition as a pullback gives $B^{\text {rep }} M$ a natural cyclic structure, and all maps in the lower two rows of the diagram are cyclic.

Lemma 3.17 The composite homomorphism $\operatorname{ker}\left(\epsilon^{\mathrm{gp}}\right) \subset B^{\mathrm{cy}} M^{\mathrm{gp}} \stackrel{\pi}{\rightarrow} B M^{\mathrm{gp}}$ is an isomorphism. Hence there is a natural isomorphism $\left(\epsilon^{\mathrm{rep}}, \pi^{\mathrm{rep}}\right): B^{\mathrm{rep}} M \cong M \times B M^{\mathrm{gp}}$, of simplicial commutative monoids under and over $M$.

Combining with the weak equivalence $B M \rightarrow B M^{\mathrm{gp}}$, we obtain a weak equivalence $M \times B M \stackrel{\simeq}{\longrightarrow} B^{\text {rep }} M$. The repletion map $B^{\text {cy }} M \rightarrow B^{\text {rep }} M$ factors as $(\epsilon, \pi): B^{\text {cy }} M \rightarrow$ $M \times B M$, followed by the latter weak equivalence.

The inclusion $\eta: M \rightarrow B^{\text {rep }} M$ factors through $\eta_{0}: M \rightarrow M \times B M$, induced by the inclusion of the base point in $B M$.

Proof The first claim is well known, since $M^{\text {gp }}$ is a group. The inverse isomorphism $B M^{\mathrm{gp}} \rightarrow \operatorname{ker}\left(\epsilon^{\mathrm{gp}}\right)$ takes $\left[m_{1}|\cdots| m_{q}\right]$ to $\left(m_{0}, m_{1}, \ldots, m_{q}\right)$ where $m_{0}=\left(m_{1} \cdots m_{q}\right)^{-1}$. The rest is clear from Lemma 3.11 .

Remark 3.18 By its definition as a pullback, $B^{\text {rep }} M$ is the simplicial commutative monoid with $q$-simplices $\left(m ; g_{0}, g_{1}, \ldots, g_{q}\right)$ with $m \in M$ and $g_{i} \in M^{\mathrm{gp}}$ for all $i$, such that $\gamma(m)=g_{0} g_{1} \cdots g_{q}$. When $\gamma$ is injective, $m$ is determined by the $\left(g_{0}, g_{1}, \ldots, g_{q}\right)$, and these are only subject to the condition that their product $g_{0} g_{1} \cdots g_{q}$ lies in the image of $\gamma$. We note that the cyclic operator on $B^{\text {rep }} M$ takes $\left(m ; g_{0}, g_{1}, \ldots, g_{q}\right)$ to $\left(m ; g_{q}, g_{0}, \ldots, g_{q-1}\right)$. This is acceptable because $M$, or rather $M^{\mathrm{gp}}$, is commutative: $\gamma(m)=g_{q} \gamma(m) g_{q}^{-1}=g_{q} g_{0} \cdots g_{q-1}$. The isomorphism $(\epsilon, \pi): B^{\text {rep }} M \cong M \times B M^{\text {gp }}$ takes $\left(m ; g_{0}, g_{1}, \ldots, g_{q}\right)$ to $\left(m,\left[g_{1}|\cdots| g_{q}\right]\right)$, so $g_{0}$ can be recovered as $\gamma(m)\left(g_{1} \cdots g_{q}\right)^{-1}$. In these terms the cyclic operator on $M \times B M^{\text {gp }}$ takes $\left(m,\left[g_{1}|\cdots| g_{q}\right]\right)$ to $\left(m,\left[\gamma(m)\left(g_{1} \cdots g_{q}\right)^{-1}\left|g_{1}\right| \cdots \mid g_{q-1}\right]\right)$, where we again use that $\gamma(m)=g_{q} \gamma(m) g_{q}^{-1}$. Note that the cyclic operator uses the group inverse in 
$M^{\mathrm{gp}}$. Hence there is in general no natural cyclic structure on $B M$ such that the weak equivalence $M \times B M \rightarrow M \times B M^{\mathrm{gp}} \cong B^{\text {rep }} M$ is a map of cyclic sets. For later work, when we study the cyclic and cyclotomic structure on log topological Hochschild homology, it will therefore be important to work with $B M^{\text {gp }}$ rather than $B M$, even if the two are naturally equivalent as spaces.

Definition 3.19 Let $\Lambda^{j-1}$ denote the cyclic $(j-1)$-simplex, represented by the object $[j-1]$ in Connes' category $\Lambda$. Its geometric realization, as a simplicial set, is $\left|\Lambda^{j-1}\right| \cong S^{1} \times \Delta^{j-1}$. The cyclic group $C_{j}$ of order $j$ acts on $[j-1]$ in $\Lambda$, hence also on $\Lambda^{j-1}$, and the induced action on $\left|\Lambda^{j-1}\right|$ balances the subgroup action on $S^{1}$ with the action on $\Delta^{j-1}$ that cyclically permutes the vertices. See Hesselholt-Madsen [28, Section 7.2].

Proposition 3.20 Let $M=\langle x\rangle=\left\{x^{j} \mid j \geq 0\right\}$ be the free commutative monoid on one generator $x$. The cyclic bar construction $B^{\mathrm{cy}} M$ decomposes as a disjoint union

$$
B^{\mathrm{cy}} M=\coprod_{j \geq 0} B^{\mathrm{cy}}(M ; j)
$$

of cyclic sets, where $B^{\text {cy }}(M ; j)=\epsilon^{-1}\left(x^{j}\right)$ consists of the simplices $\left(m_{0}, \ldots, m_{q}\right)$ with $m_{0} \cdots m_{q}=x^{j}$. Here $B^{\text {cy }}(M ; 0)=*$ is a single point, while for $j \geq 1$ there is a cyclic isomorphism $\Lambda^{j-1} / C_{j} \cong B^{\mathrm{cy}}(M ; j)$. After geometric realization there is an $S^{1}$-equivariant homeomorphism

$$
S^{1} \times C_{j} \Delta^{j-1} \stackrel{\cong}{\longrightarrow} B^{\mathrm{cy}}(M ; j) .
$$

Hence there is an $S^{1}$-equivariant deformation retraction

$$
B^{\mathrm{cy}} M \stackrel{\simeq}{\rightarrow} * \sqcup \coprod_{j \geq 1} S^{1}(j),
$$

where $S^{1}(j)=S^{1} / C_{j}$. The 1-simplex $\left(x^{j-1}, x\right)$ forms a closed loop at $\left(x^{j}\right)$ that maps by an equivalence to $S^{1}(j)$.

Proof This follows from the proof of Hesselholt [27, 2.2.3]. For $j \geq 1$ the $(j-1)-$ simplex $(x, x, \ldots, x)$ generates $B^{\mathrm{cy}}(M ; j)$ as a cyclic set. Hence there is a surjective cyclic map $\Lambda^{j-1} \rightarrow B^{\text {cy }}(M ; j)$. The restriction of the canonical $S^{1}$-action on $\left|B^{\text {cy }} M\right|$ to $C_{j} \subset S^{1}$ acts on the $(j-1)$-simplices by cyclic permutation, and fixes $(x, x, \ldots, x)$. Hence the cyclic map factors over $\Lambda^{j-1} / C_{j}$. There are no further relations in $B^{\text {cy }}(M ; j)$, giving the asserted cyclic isomorphism and $S^{1}$-equivariant homeomorphism. The simplex $\Delta^{j-1}$ is $C_{j}$-equivariantly contractible to its barycenter, giving the asserted $S^{1}$-equivariant homotopy equivalence. 
Proposition 3.21 Let $M=\langle x\rangle$, with group completion $M^{\mathrm{gp}}=\left\langle x, x^{-1}\right\rangle=\left\{x^{j} \mid\right.$ $j \in \mathbb{Z}\}$. The cyclic bar construction $B^{\text {cy }} M^{\text {gp }}$ decomposes as a disjoint union

$$
B^{\mathrm{cy}} M^{\mathrm{gp}}=\coprod_{j \in \mathbb{Z}} B^{\mathrm{cy}}\left(M^{\mathrm{gp}} ; j\right) \simeq \coprod_{j \in \mathbb{Z}} S^{1}(j)
$$

of cyclic sets, where $B^{\mathrm{cy}}\left(M^{\mathrm{gp}} ; j\right)=\left(\epsilon^{\mathrm{gp}}\right)^{-1}\left(x^{j}\right)$, and $\left(x^{j-1}, x\right)$ forms a closed loop mapping by an equivalence to $S^{1}(j)$. Hence

$$
B^{\text {rep }} M=\coprod_{j \geq 0} B^{\text {cy }}\left(M^{\mathrm{gp}} ; j\right) \simeq \coprod_{j \geq 0} S^{1}(j)
$$

and the repletion map $B^{\text {cy }} M \rightarrow B^{\text {rep }} M$ decomposes as the disjoint union of the inclusions

$$
B^{\text {cy }}(M ; j) \rightarrow B^{\text {cy }}\left(M^{\mathrm{gp}} ; j\right)
$$

for $j \geq 0$. For each $j \geq 1$, this inclusion is an $S^{1}$-equivariant homotopy equivalence. For $j=0$, the map

$$
*=B^{\mathrm{cy}}(M ; 0) \rightarrow B^{\mathrm{cy}}\left(M^{\mathrm{gp}} ; 0\right)
$$

identifies the source with the $S^{1}$-fixed points of the target.

There is a cyclic isomorphism $B^{\text {cy }}\left(M^{\mathrm{gp}} ; 0\right) \cong B M^{\mathrm{gp}}$, where $B M^{\mathrm{gp}} \simeq S^{1}(0)$ has the cyclic structure taking $\left[m_{1}|\cdots| m_{q}\right]$ to $\left[\left(m_{1} \cdots m_{q}\right)^{-1}\left|m_{1}\right| \cdots \mid m_{q-1}\right]$. The associated circle action

$$
S^{1} \times B M^{\mathrm{gp}} \rightarrow B M^{\mathrm{gp}}
$$

is homotopic to the trivial action. Furthermore, for each finite subgroup $C_{r} \subset S^{1}$ there is a homeomorphism $B^{\mathrm{cy}}\left(M^{\mathrm{gp}} ; 0\right) \cong B^{\mathrm{cy}}\left(M^{\mathrm{gp}} ; 0\right)^{C_{r}}$, which is equivariant with respect to the canonical group homomorphism $S^{1} \rightarrow S^{1} / C_{r}$. Hence there is an $S^{1}$-equivariant homotopy pushout square

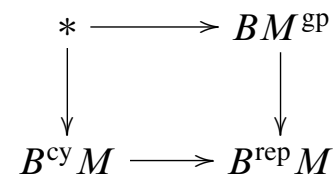

of simplicial sets, where $B M^{\mathrm{gp}} \simeq S^{1}(0)$.

Proof For each $j \geq 0$, the projection $\pi: B^{\text {cy }} M^{\text {gp }} \rightarrow B M^{\text {gp }}$ restricts to a cyclic isomorphism

$$
B^{\mathrm{cy}}\left(M^{\mathrm{gp}} ; j\right) \stackrel{\cong}{\rightarrow} B M^{\mathrm{gp}},
$$

when the target is given the cyclic structure that takes $\left[m_{1}|\cdots| m_{q}\right]$ to

$$
\left[x^{j}\left(m_{1} \cdots m_{q}\right)^{-1}\left|m_{1}\right| \cdots \mid m_{q-1}\right] .
$$


The closed 1-simplex $\left(x^{j-1}, x\right) \leftrightarrow[x]$ induces a homotopy equivalence $S^{1} \rightarrow B M^{\mathrm{gp}}$, and the circle action on $B^{\mathrm{gp}} M$ is compatible, up to homotopy, with the circle action $S^{1} \times S^{1} \rightarrow S^{1}$ given by $(z, w) \mapsto z^{j} w$. See Loday [42, 7.3.4, 7.4.5].

For $j \geq 1$, the circle action on $B^{\text {cy }}(M ; j) \simeq S^{1}(j)$ takes the 0 -simplex $\left(x^{j}\right)$ once around the 1-simplex $\left(1, x^{j}\right)$, which deformation retracts to a loop winding $j$ times around $S^{1}(j)$. By inspection of the simplicial structure, the loop $\left(1, x^{j}\right)$ is homotopic to the composite of $j$ copies of the loop $\left(x^{j-1}, x\right)$. Hence the map $S^{1} \simeq B^{\text {cy }}(M ; j) \rightarrow$ $B^{\text {cy }}\left(M^{\mathrm{gp}} ; j\right) \simeq S^{1}$ has degree 1 , and is a homotopy equivalence, for all $j \geq 1$.

To check that this map is an $S^{1}$-equivariant equivalence, we check that the map of $H$-fixed points is a homotopy equivalence for each closed subgroup $H \subseteq S^{1}$. The $S^{1}$-fixed points of a cyclic set $Z$ consists of the 0 -simplices $z$ with $t_{1} s_{0} z=s_{0} z$. In $B^{\text {cy }}(M ; j)$ and $B^{\text {cy }}\left(M^{\text {gp }} ; j\right)$ the only 0 -simplex is $z=\left(x^{j}\right)$, with $s_{0} z=\left(1, x^{j}\right)$ and $t_{1} s_{0} z=\left(x^{j}, 1\right)$, so both fixed point spaces are empty for $j \geq 1$, whereas for $j=0$ both fixed point spaces consist of the single point (1).

To study the fixed points for finite subgroups $C_{r} \subset S^{1}$, we use the $r$-fold edgewise subdivision functor $Z \mapsto \operatorname{sd}_{r} Z$ of Bökstedt-Hsiang-Madsen [15, Section 1], with $\left(\operatorname{sd}_{r} Z\right)_{q}=Z_{r(q+1)-1}$ for $q \geq 0$. Recall that there is an $S^{1}$-equivariant homeomorphism $D_{r}:\left|\operatorname{sd}_{r} Z\right| \cong|Z|$ for cyclic sets $Z$, and the $C_{r}$-action on $\left|\operatorname{sd}_{r} Z\right|$ is induced by a simplicial action on $\operatorname{sd}_{r} Z$. There is a simplicial isomorphism $B_{\bullet}^{\mathrm{cy}} M \cong$ $\left(\operatorname{sd}_{r} B_{\bullet}^{\text {cy }} M\right)^{C_{r}}$ given by the $r$-th power map

$$
\Delta_{r}:\left(m_{0}, \ldots, m_{q}\right) \mapsto\left(m_{0}, \ldots, m_{q}, \ldots, m_{0}, \ldots, m_{q}\right)
$$

(repeating its argument $r$ times), which leads to the chain of homeomorphisms

$$
B^{\mathrm{cy}} M \stackrel{\left|\Delta_{r}\right|}{\cong}\left|\left(\operatorname{sd}_{r} B_{\bullet}^{\mathrm{cy}} M\right)^{C_{r}}\right| \cong\left|\operatorname{sd}_{r} B_{\bullet}^{\mathrm{cy}} M\right|^{C_{r}} \stackrel{D_{r}^{C_{r}}}{\cong}\left(B^{\mathrm{cy}} M\right)^{C_{r}} .
$$

The composite homeomorphism is equivariant with respect to the canonical group homomorphism $S^{1} \rightarrow S^{1} / C_{r}$. Since $\epsilon\left(\Delta_{r}(z)\right)=\epsilon(z)^{r}$, this homeomorphism restricts to a homeomorphism

$$
B^{\mathrm{cy}}(M ; i) \stackrel{\cong}{\longrightarrow} B^{\mathrm{cy}}(M ; r i)^{C_{r}},
$$

whereas $B^{\text {cy }}(M ; j)$ has no $C_{r}$-fixed points when $r \nmid j$.

Similar results hold for $M^{\text {gp }}$, so by naturality we can identify

$$
\gamma^{C_{r}}: B^{\mathrm{cy}}(M ; j)^{C_{r}} \rightarrow B^{\mathrm{cy}}\left(M^{\mathrm{gp}} ; j\right)^{C_{r}}
$$

with the homotopy equivalence $B^{\text {cy }}(M ; i) \rightarrow B^{\text {cy }}\left(M^{\mathrm{gp}} ; i\right)$ for $j=r i$, and with the trivial equivalence $\varnothing \rightarrow \varnothing$ for $r \nmid j$. Hence $B^{\text {cy }}(M ; j) \rightarrow B^{\text {cy }}\left(M^{\text {gp }} ; j\right)$ is an $S^{1}-$ equivariant homotopy equivalence, for $j \geq 1$. 
Definition 3.22 Let $A$ be a commutative ring. Suppose first that $A$ is flat over $\mathbb{Z}$. The Hochschild homology of $A$ is the simplicial ring $\operatorname{HH}(A)=S_{\bullet}^{1} \otimes A$, with

$$
\mathrm{HH}(A)_{q} \cong A \otimes A \otimes \cdots \otimes A
$$

$((q+1)$ copies of $A)$ in simplicial degree $q$. The Hochschild homology groups of $A$ are the homotopy groups $\mathrm{HH}_{i}(A)=\pi_{i} \mathrm{HH}(A)$. The natural maps $\eta: A \rightarrow \mathrm{HH}(A)$ and $\epsilon: \mathrm{HH}(A) \rightarrow A$ make $\mathrm{HH}(A)$ a simplicial object in $A / \mathcal{C}$ Ring $/ A$, and $\epsilon$ makes $\mathrm{HH}(A)$ a cyclic object in $\mathcal{C} \mathcal{R}$ ing / $A$. If $A$ is not flat over $\mathbb{Z}$, we replace $A$ by a $\mathbb{Z}$-flat simplicial resolution, form $\mathrm{HH}(-)$ degreewise, and pass to the diagonal of the resulting bisimplicial ring.

Definition 3.23 Let $(A, M, \alpha)$ be a pre-log ring. There is a natural pre-log structure

$$
B^{\mathrm{cy}} M \rightarrow(\mathrm{HH}(A), \cdot)
$$

with left adjoint $S_{\bullet}^{1} \otimes \bar{\alpha}: \mathbb{Z}\left[B^{\text {cy }} M\right] \rightarrow \mathrm{HH}(A)$. It makes $\left(\mathrm{HH}(A), B^{\mathrm{cy}} M\right)$ a simplicial object in $(A, M) / \operatorname{Pre} \mathcal{L} o g /(A, M)$, and a cyclic object in $\operatorname{Pre} \mathcal{L} \log ^{\text {vsur }} /(A, M)$.

Suppose first that $A$ is flat over $\mathbb{Z}[M]$, so that $\operatorname{HH}(A)$ is flat over $\operatorname{HH}(\mathbb{Z}[M])=$ $\mathbb{Z}\left[B^{\mathrm{cy}} M\right]$. By definition, the log Hochschild homology $\left(\mathrm{HH}(A, M), B^{\text {rep }} M, \xi\right)$ of $(A, M)$ is the replete simplicial pre-log ring $S_{\bullet}^{1} \otimes^{\text {rep }}(A, M)$. Here $\operatorname{HH}(A, M)$ is given by the pushout square

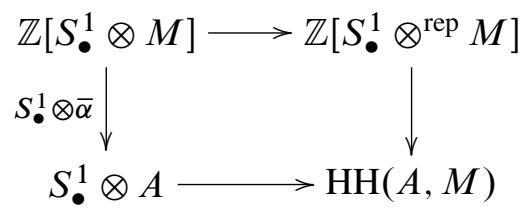

of simplicial commutative rings, which we can rewrite as the pushout square

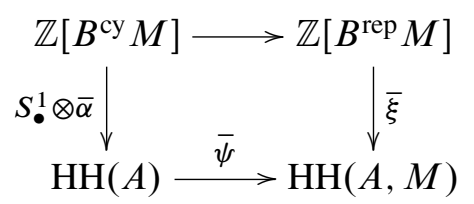

in the same category. The pre-log structure map

$$
\xi: B^{\text {rep }} M \rightarrow(\mathrm{HH}(A, M), \cdot)
$$

is right adjoint to the right hand vertical map $\bar{\xi}$. The log Hochschild homology groups of $(A, M)$ are the homotopy groups $\mathrm{HH}_{i}(A, M)=\pi_{i} \mathrm{HH}(A, M)$.

So $\mathrm{HH}(A, M)$ is naturally a simplicial pre-log ring under and over $(A, M)$, and a cyclic pre-log ring over $(A, M)$. The comparison homomorphism $\bar{\psi}: \mathrm{HH}(A) \rightarrow \operatorname{HH}(A, M)$ 
is a morphism in each of these two categories. If $A$ is not flat over $\mathbb{Z}[M]$, we replace $A$ by a $\mathbb{Z}[M]$-flat simplicial resolution, form $\mathrm{HH}(-, M)$ degreewise, and pass to the diagonal of the resulting bisimplicial ring.

Remark 3.24 We can also rewrite the pushout squares in Definition 3.23 as follows

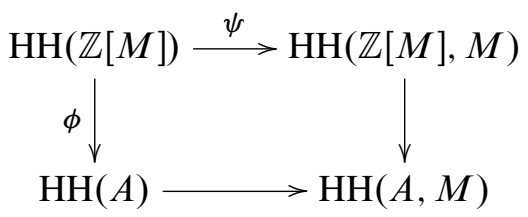

where $\mathbb{Z}[M]$ has the canonical pre-log structure. In this sense the log Hochschild homology of the canonical pre-log rings $(\mathbb{Z}[M], M)$ (together with the Hochschild homology of ordinary rings) determines the log Hochschild homology of general prelog rings. It can also be convenient to base change the top row of this square along $\bar{\alpha}: \mathbb{Z}[M] \rightarrow A$, to obtain a pushout square

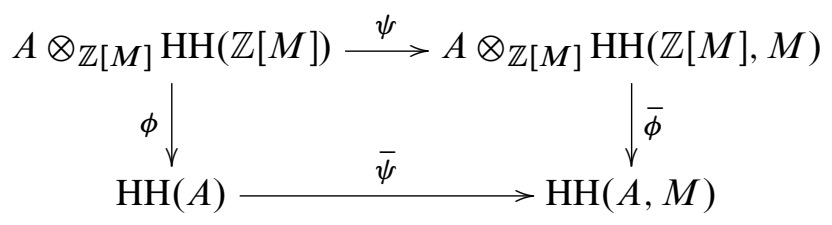

of simplicial commutative $A$-algebras. Here $A \otimes_{\mathbb{Z}[M]} \mathrm{HH}(\mathbb{Z}[M], M) \cong A \otimes \mathbb{Z}\left[B M^{\mathrm{gp}}\right]$.

\section{Logarithmic Kähler differentials}

We return to a review of the log Kähler forms in algebra, modifying Kato's discussion [35, Sections 1, 3] to emphasize Dan Quillen's view on commutative ring derivations in terms of abelian group objects [60, Section 2]. Again, we restrict attention to commutative rings, but the generalization to commutative $R$-algebras over a base commutative ring $R$ is easy.

Definition 4.1 Let $A$ be a commutative ring and let $J$ be a left $A$-module. Since $A$ is commutative, we can also think of $J$ as a right $A$-module, with $j a=a j$. The square-zero extension $A \oplus J$ is the commutative ring with multiplication map

$$
(A \oplus J) \times(A \oplus J) \rightarrow(A \oplus J)
$$

that takes $\left(a_{1} \oplus j_{1}, a_{2} \oplus j_{2}\right)$ to $a_{1} a_{2} \oplus\left(j_{1} a_{2}+a_{1} j_{2}\right)$. The obvious projection $\epsilon: A \oplus J \rightarrow A$ makes $A \oplus J$ a commutative ring over $A$, with augmentation ideal $J$ having the zero multiplication $J \times J \rightarrow J$. 
Remark 4.2 The inclusion $\eta: A \rightarrow A \oplus J$ taking $a$ to $a \oplus 0$, the multiplication $\mu: A \oplus J \oplus J \cong(A \oplus J) \times_{A}(A \oplus J) \rightarrow A \oplus J$ taking $a \oplus j_{1} \oplus j_{2}$ to $a \oplus\left(j_{1}+j_{2}\right)$, and the conjugation $\chi: A \oplus J \rightarrow A \oplus J$ taking $a \oplus j$ to $a \oplus(-j)$, make $A \oplus J$ an abelian group object in the category $\mathcal{C}$ Ring / $A$ of commutative rings over $A$. As Quillen remarks, the functor $J \mapsto A \oplus J$ is an equivalence from the category $\operatorname{Mod}_{A}$ of $A$-modules to the category $(\mathcal{C R} i n g / A)_{\mathrm{ab}}$ of abelian group objects in $\mathcal{C} \operatorname{Ring} / A$.

Definition 4.3 Let $A$ and $J$ be as above. The derivations of $A$ with values in $J$ is the abelian group

$$
\operatorname{Der}(A, J)=(\mathcal{C} \mathcal{R} i n g / A)(A, A \oplus J)
$$

of ring homomorphisms $D: A \rightarrow A \oplus J$ over $A$. These all have the form $D(a)=$ $a \oplus d(a)$ where $d(a b)=d(a) b+a d(b)$, so the additive group homomorphism $d: A \rightarrow$ $J$ is a derivation in the more elementary sense. The Kähler differentials of $A$ is the $A$-module

$$
\Omega_{A}^{1}=A\{d a \mid a \in A\} /(d(a b)=(d a) b+a(d b))
$$

generated by symbols $d a$ for $a \in A$, subject to the relations $d(a b)=(d a) b+a(d b)$ for all $a, b \in A$. It corepresents derivations, in the following sense.

Lemma 4.4 The universal derivation $D: A \rightarrow A \oplus \Omega_{A}^{1}$, taking $a$ to $D(a)=a \oplus d a$, induces a natural isomorphism

$$
\operatorname{Hom}_{A}\left(\Omega_{A}^{1}, J\right) \cong \operatorname{Der}(A, J) .
$$

Lemma 4.5 Let $g: C \rightarrow A$ be a homomorphism of commutative rings, and let $J$ be an $A$-module. Write $g^{\#} J$ for $J$ viewed as a $C$-module via $g$. Composition with $g \oplus \mathrm{id}: C \oplus J \rightarrow A \oplus J$ induces an isomorphism

$$
\operatorname{Der}\left(C, g^{\#} J\right) \cong(\mathcal{C} \mathcal{R} i n g / A)(C, A \oplus J) .
$$

Proof This is clear, since $\epsilon: C \oplus J \rightarrow C$ is the pullback of $\epsilon: A \oplus J \rightarrow A$ along $g$.

Lemma 4.6 Let $M=\langle X\rangle$ be the free commutative monoid on a set $X$. Then

$$
\Omega_{\mathbb{Z}[M]}^{1} \cong \mathbb{Z}[M] \otimes M^{\mathrm{gp}}
$$

is the free $\mathbb{Z}[M]$-module induced up from $M^{\mathrm{gp}}$, with $d x$ corresponding to $1 \otimes \gamma(x)$, for all $x \in X \subset M$. 
Proof For each $\mathbb{Z}[M]$-module $J$, there are natural isomorphisms

$$
\begin{aligned}
\operatorname{Der}(\mathbb{Z}[M], J) & \cong\{\text { functions } d: M \rightarrow J \text { with } d(a b)=d(a) b+a d(b)\} \\
& \cong\{\text { functions } X \rightarrow J\} \\
& \cong \mathcal{C} \mathcal{M} \operatorname{on}(M,(J,+)) \cong \mathcal{A} b\left(M^{\mathrm{gp}},(J,+)\right) \\
& \cong \operatorname{Hom}_{\mathbb{Z}[M]}\left(\mathbb{Z}[M] \otimes M^{\mathrm{gp}}, J\right) .
\end{aligned}
$$

Hence $\mathbb{Z}[M] \otimes M^{\mathrm{gp}}$ corepresents derivations of $\mathbb{Z}[M]$.

Remark 4.7 When extended to simplicial commutative rings, the functor $A \mapsto$ $\operatorname{Der}(A, J)$ admits homotopical right derived functors, known as the André-Quillen cohomology groups $D^{q}(A, J)$; see Quillen [60, Section 4]. These are corepresented as the cohomology groups $H^{q}\left(\operatorname{Hom}_{A}\left(\mathbb{L} \Omega_{A}^{1}, J\right)\right)$ of (the chain complex associated to) the simplicial $A$-module $\mathbb{L} \Omega_{A}^{1}=A \otimes_{P_{\bullet}} \Omega_{P_{\bullet}}^{1}$, known as the cotangent complex, where $P_{\bullet} \stackrel{\simeq}{\longrightarrow} A$ is a cofibrant simplicial commutative ring resolution of $A$. (Cofibrant effectively means that $P_{\bullet}$ is a free commutative ring, ie, a polynomial ring, in each simplicial degree.) The homology groups of the cotangent complex are the André-Quillen homology groups $D_{q}(A, J)=H_{q}\left(J \otimes_{A} \mathbb{L} \Omega_{A}^{1}\right)$. As special cases, $D^{0}(A, J) \cong \operatorname{Der}(A, J)$ and $D_{0}(A, J) \cong J \otimes_{A} \Omega_{A}^{1}$. When we pass from the algebraic to the topological context in the next sections, we will automatically be working with mapping spaces that incorporate these derived functors. Therefore the natural generalization of the Kähler differentials will be the topological form of the cotangent complex $\mathbb{L} \Omega_{A}^{1}$, namely the topological André-Quillen homology spectrum $\operatorname{TAQ}(A)$.

Lemma 4.8 Let $M$ be a commutative monoid, and let $F_{\bullet} \stackrel{\simeq}{\longrightarrow} M$ be a cofibrant simplicial commutative monoid resolution of $M$. Then

$$
\mathbb{L} \Omega_{\mathbb{Z}[M]}^{1} \simeq \mathbb{Z}[M] \otimes F_{\bullet}^{\mathrm{gp}} .
$$

Proof Cofibrancy effectively means that $F_{\bullet}$ is a free commutative monoid in each simplicial degree. Then $P_{\bullet}=\mathbb{Z}\left[F_{\bullet}\right] \stackrel{\simeq}{\longrightarrow} \mathbb{Z}[M]$ is a cofibrant simplicial commutative ring resolution of $\mathbb{Z}[M]$, and $\Omega_{P_{\bullet}}^{1} \cong \mathbb{Z}\left[F_{\bullet}\right] \otimes F_{\bullet}^{\mathrm{gp}}$, by Lemma 4.6. Hence

$$
\mathbb{L} \Omega_{\mathbb{Z}[M]}^{1} \simeq \mathbb{Z}[M] \otimes_{\mathbb{Z}\left[F_{\bullet}\right]}\left(\mathbb{Z}\left[F_{\bullet}\right] \otimes F_{\bullet}^{\mathrm{gp}}\right) \cong \mathbb{Z}[M] \otimes F_{\bullet}^{\mathrm{gp}},
$$

where $F_{\bullet}^{\mathrm{gp}}$ denotes the degreewise group completion of $F_{\bullet}$.

Remark 4.9 In the notation of Lemma 4.6, $d m \in \Omega_{\mathbb{Z}[M]}^{1}$ does typically not correspond to $1 \otimes \gamma(m) \in \mathbb{Z}[M] \otimes M^{\text {gp }}$ when $m \in M \backslash X$. For example, $d\left(x^{2}\right)=2 x d x$ corresponds to $2 x \otimes \gamma(x)$ rather than $1 \otimes \gamma\left(x^{2}\right)$, when $x \in X$. It follows that the simplicial $\mathbb{Z}[M]$-module structure on $\mathbb{Z}[M] \otimes F_{\bullet}^{\mathrm{gp}}$ in Lemma 4.8 is usually not induced 
up from the simplicial abelian group structure on $F_{\bullet}^{\mathrm{gp}}$. It would be if the simplicial

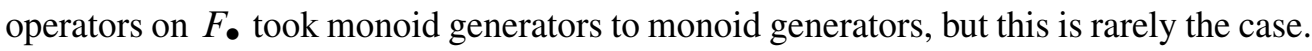
For example, if a face of $y \in F_{1}$ is $x^{2} \in F_{0}$, where $y$ and $x$ are monoid generators, then the corresponding face of $1 \otimes \gamma(y)$ is $2 x \otimes \gamma(x)$, not $1 \otimes \gamma\left(x^{2}\right)$.

The zero-th homotopy group of $\mathbb{L} \Omega_{\mathbb{Z}[M]}^{1}$ recovers the Kähler differentials $\Omega_{\mathbb{Z}[M]}^{1}$, which also does not need to be an extended $\mathbb{Z}[M]$-module. It will be a finitely generated projective $\mathbb{Z}[M]$-module when $\mathbb{Z}[M]$ is smooth over $\mathbb{Z}$, but as we have just discussed, the face maps $\mathbb{Z}[M] \otimes F_{1}^{\mathrm{gp}} \rightrightarrows \mathbb{Z}[M] \otimes F_{0}^{\mathrm{gp}}$ with coequalizer $\pi_{0} \mathbb{L} \Omega_{\mathbb{Z}[M]}^{1}$ are not extended $\mathbb{Z}[M]$-module maps in general. In the same way, the topological André-Quillen homology $\operatorname{TAQ}(S[M])$ to be discussed in Definition 10.3, will not in general be an extended $S[M]$-module, even if this is so when $M$ is a grouplike or free commutative $\mathcal{I}$-space monoid. See Remark 10.11 .

Remark 4.10 To define log derivations and log Kähler differentials, we should determine the abelian group objects in a category of log rings over a fixed $\log \operatorname{ring}(A, M)$. A maximal choice is the category $\log /(A, M)$ of all $\log$ rings over $A$. A minimal choice is the subcategory $\operatorname{str} \log /(A, M)$ of log rings with a strict homomorphism to $(A, M)$, and strict homomorphisms between these. An intermediate choice, and probably the most interesting one, is the category $\log ^{\text {rep }} /(A, M)$ of replete log rings over $(A, M)$.

The forgetful functors from $\log$ to $\mathcal{P}$ re $\mathcal{L} o g, \mathcal{C}$ ing and $\mathcal{C}$ Mon are right adjoints, hence preserve limits. It follows that the categorical product in $\mathcal{L} o g /(A, M)$ of two log rings $\left(B_{1}, N_{1}\right)$ and $\left(B_{2}, N_{2}\right)$, both over $(A, M)$, is the log ring $\left(B_{1} \times_{A} B_{2}, N_{1} \times_{M} N_{2}\right)$ over $(A, M)$. Here $B_{1} \times_{A} B_{2} \subset B_{1} \times B_{2}$ and $N_{1} \times{ }_{M} N_{2} \subset N_{1} \times N_{2}$ are the usual fiber products.

When both augmentations $\left(B_{i}, N_{i}\right) \rightarrow(A, M)$ are strict, and both projections

$$
\left(B_{1} \times_{A} B_{2}, N_{1} \times_{M} N_{2}\right) \rightarrow\left(B_{i}, N_{i}\right)
$$

are strict, then $\left(B_{1} \times_{A} B_{2}, N_{1} \times_{M} N_{2}\right)$ is the product of $\left(B_{1}, N_{1}\right)$ and $\left(B_{2}, N_{2}\right)$ in the subcategory $\operatorname{str} \log /(A, M)$. When both augmentations $\left(B_{i}, N_{i}\right) \rightarrow(A, M)$ are replete, the fiber product $\left(B_{1} \times_{A} B_{2}, N_{1} \times_{M} N_{2}\right)$ is replete over $(A, M)$ (by Lemma $3.11)$, so this is the product of $\left(B_{1}, N_{1}\right)$ and $\left(B_{2}, N_{2}\right)$ in $\log ^{\text {rep }} /(A, M)$.

Definition 4.11 Let $(A, M)$ be a log ring and let $J$ be an $A$-module. The squarezero extension $\left(A \oplus J, \eta^{*} M\right)$ is the log ring with $A \oplus J$ as its underlying commutative ring, and the inverse image $\eta^{*} M$ of $M$ along the inclusion $\eta: A \rightarrow A \oplus J$ as its underlying commutative monoid. The projection $\epsilon: A \oplus J \rightarrow A$ induces a strict 
homomorphism $\left(\epsilon, \epsilon^{b}\right):\left(A \oplus J, \eta^{*} M\right) \rightarrow\left(A, \epsilon^{*} \eta^{*} M\right) \cong(A, M)$, since $\epsilon \eta=\mathrm{id}_{A}$, which makes $\left(A \oplus J, \eta^{*} M\right)$ an object of $\operatorname{str} \mathcal{L} \operatorname{og} /(A, M)$.

Lemma 4.12 Let $(A, M)$ and $J$ be as above. There is an isomorphism

$$
M \times(J,+) \cong \eta^{*} M
$$

of commutative monoids, where $(J,+)$ denotes the underlying additive monoid of $J$. Under this isomorphism, the $\log$ structure map $\eta^{*} \alpha: \eta^{*} M \rightarrow A \oplus J$ takes $(m, j)$ to $\alpha(m) \cdot(1 \oplus j)=\alpha(m) \oplus \alpha(m) \cdot j$.

Proof We have a commutative diagram

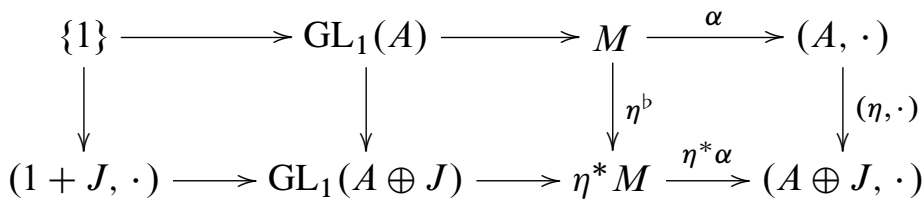

of commutative monoids. The preimage of $\mathrm{GL}_{1}(A \oplus J) \subset(A \oplus J, \cdot)$ in $(A, \cdot)$ is $\mathrm{GL}_{1}(A)$, so its preimage in $M$ is also isomorphic to $\mathrm{GL}_{1}(A)$, since $(M, \alpha)$ is a log structure. It follows that the middle square is the pushout defining the logification $\eta^{*} M$. The left hand square is also a pushout, since $\mathrm{GL}_{1}(A \oplus J) \cong \mathrm{GL}_{1}(A) \times(1+J, \cdot)$. This gives an isomorphism $M \times(1+J, \cdot) \cong \eta^{*} M$. When combined with the monoid isomorphism $(J,+) \cong(1+J, \cdot)$ that takes $j \in J$ to $1+j \in 1+J$, we obtain the isomorphism of the lemma.

Lemma 4.13 Let $(A, M)$ be a $\log$ ring. The functor taking an $A$-module $J$ to the square-zero extension $\left(A \oplus J, \eta^{*} M\right)$ is an equivalence from the category $\operatorname{Mod}_{A}$ of $A$-modules to the category of $(\operatorname{str} \mathcal{L} o g /(A, M))_{\mathrm{ab}}$ of abelian group objects in $\operatorname{str} \mathcal{L} o g /(A, M)$.

Proof The two projections from

$$
(A \oplus J \oplus J, M \times J \times J) \cong\left((A \oplus J) \times_{A}(A \oplus J), \eta^{*} M \times_{M} \eta^{*} M\right)
$$

to $\left(A \oplus J, \eta^{*} M\right) \cong(A \oplus J, M \times J)$ are strict, so $\left((A \oplus J) \times_{A}(A \oplus J), \eta^{*} M \times_{M} \eta^{*} M\right)$ is the product of $\left(A \oplus J, \eta^{*} M\right)$ with itself in $\operatorname{str} \log /(A, M)$.

The inverse image of $M$ along $\eta: A \rightarrow A \oplus J$, the inverse image of $\eta^{*} M \times_{M} \eta^{*} M$ along $\mu:(A \oplus J) \times_{A}(A \oplus J) \rightarrow(A \oplus J)$, and the inverse image of $\eta^{*} M$ along $\chi: A \oplus J \rightarrow A \oplus J$, are all canonically isomorphic to $\eta^{*} M$. Hence the abelian group object structure maps $\eta, \mu$ and $\chi$ of $A \oplus J$ in $\mathcal{C} \mathcal{R} i n g / A$ are all covered 
by strict homomorphisms of log rings $\left(\eta, \eta^{b}\right),\left(\mu, \mu^{b}\right)$ and $\left(\chi, \chi^{b}\right)$, specifying how $\left(A \oplus J, \eta^{*} M\right)$ is an abelian group object in $\operatorname{str} \mathcal{L} o g /(A, M)$.

Conversely, an abelian group object $(B, N)$ in $\operatorname{str} \log /(A, M)$ must map by the forgetful functor to an abelian group object in $\mathcal{C} \mathcal{R} i n g$, so $B \cong A \oplus J$ must be a squarezero extension. For the unit homomorphism $\left(\eta, \eta^{\mathrm{b}}\right):(A, M) \rightarrow(B, N)$ to be strict, we must have $N \cong \eta^{*} M$. Hence $\operatorname{Mod}_{A} \rightarrow(\operatorname{str} \mathcal{L} o g /(A, M))_{\text {ab }}$ is an equivalence of categories.

Remark 4.14 By the previous lemma, the category of abelian group objects in str $\log /(A, M)$ does not depend on the $\log$ structure on $A$. It is plausible that the larger category of abelian group objects in $\log /(A, M)$, where the morphisms are not required to be strict, provides a more interesting category of "log modules" over $(A, M)$. In this case, the underlying commutative ring of an abelian group object $(B, N)$ in $\mathcal{L} o g /(A, M)$ must still be a square-zero extension $B \cong A \oplus J$, while the underlying commutative monoid must be an abelian group object $N$ in $\mathcal{C M o n} / M$.

The latter objects must have the form $\epsilon: N \rightarrow M$, where $\epsilon^{-1}(m) \subset N$ is an abelian group for each $m \in M$, and the monoidal pairing $N \times N \rightarrow N$ is given by group homomorphisms $\epsilon^{-1}\left(m_{1}\right) \times \epsilon^{-1}\left(m_{2}\right) \rightarrow \epsilon^{-1}\left(m_{1} m_{2}\right)$, for $m_{1}, m_{2} \in M$. For example, each abelian group $K$ determines an abelian group object $N=M \times K$ in $\mathcal{C M o n} / M$, with structure maps $\epsilon(m, k)=m, \eta(m)=(m, e), \mu\left(m, k_{1}, k_{2}\right)=\left(m, k_{1} k_{2}\right)$ and $\chi(m, k)=\left(m, k^{-1}\right)$. However, in the current generality there are also abelian group objects that do not have this form. For example, if $M \cong\left(\mathbb{N}_{0},+\right)$ and $K \cong(\mathbb{Z},+)$, the commutative submonoid $N \subset M \times K$ with $\epsilon^{-1}(e)=\{e\}$ and $\epsilon^{-1}(m)=K$ for $m \neq e$, is an abelian group object in $\mathcal{C M o n} / M$. In this example, $N$ is integral but not finitely generated. Replacing $K \cong(\mathbb{Z},+)$ by $K \cong \mathbb{Z} / 2$ we get a fine (= finitely generated and integral) example $N$ that is not saturated.

It therefore appears that the full category $(\mathcal{L} o g /(A, M))_{\text {ab }}$ is rather complicated. By restricting attention to fs (= fine and saturated) monoids $N$ and $M$, or by working only with $N$ that are replete over $M$, one may ensure that the abelian group objects in the restricted subcategory of $\mathcal{C M o n} / M$ all have the form $N=M \times K$, for an abelian group $K$. This seems to lead to more manageable categories $\left(\mathcal{L}_{0} g^{\mathrm{fs}} /(A, M)\right)_{\mathrm{ab}}$ and

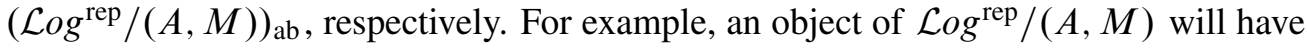
the form $(A \oplus J, M \times K, \gamma)$ for some $A$-module $J$, some abelian group $K$ and some pre-log structure

$$
\gamma: M \times K \rightarrow(A \oplus J, \cdot) .
$$

This leads to questions like which $\gamma$ specify (replete) log structures, and which objects $(A \oplus J, M \times K, \gamma)$ are abelian group objects in $\log ^{\text {rep }} /(A, M)$. We think these 
abelian objects in replete log rings over $(A, M)$ constitute a good candidate for a category of $\log$ modules over $(A, M)$.

In the topological context, it is more natural to consider stable objects, or spectra, rather than abelian group objects. The slogan is that "stabilization is abelianization", as seen eg in Schwede [72]. We view replete log rings under and over $(A, M)$ as a based (= pointed) category, and can form nonempty coproducts within this category, as in Definition 3.12. Passing to simplicial replete log rings under and over $(A, M)$, we can form tensor products with nonempty simplicial sets, and tensor product with the simplicial circle $S_{\bullet}^{1}$ specifies a suspension functor on this category. The stable category of (symmetric) spectra

$$
\mathcal{S} p^{\Sigma}\left((A, M) / \mathcal{L}^{\mathrm{rep}} /(A, M)\right)
$$

of simplicial replete log rings under and over $(A, M)$, with respect to this suspension functor, appears to be the best algebraic category of $\log$ modules over $(A, M)$. In joint work with Steffen Sagave we are investigating the Quillen $K$-theory [61] of this category, and its relation to the Quillen $K$-theory of the localization $A\left[M^{-1}\right]=$ $A \otimes_{\mathbb{Z}[M]} \mathbb{Z}\left[M^{\mathrm{gp}}\right]$.

Definition 4.15 Let $(A, M)$ be a $\log$ ring and $J$ an $A$-module. The $\log$ derivations of $(A, M)$ with values in $J$ is the abelian group

$$
\operatorname{Der}((A, M), J)=(\mathcal{L} o g /(A, M))\left((A, M),\left(A \oplus J, \eta^{*} M\right)\right)
$$

of homomorphisms $\left(D, D^{\mathrm{b}}\right):(A, M) \rightarrow\left(A \oplus J, \eta^{*} M\right)$ of $\log \operatorname{rings}$ over $(A, M)$.

More precisely, we should form the abelian group of strict log homomorphisms over $(A, M)$, but this is no restriction, as the following lemma shows.

Lemma 4.16 Every $\log$ homomorphism $\left(D, D^{b}\right):(A, M) \rightarrow\left(A \oplus J, \eta^{*} M\right)$ over $(A, M)$ is strict.

Proof The inverse images $D^{*} M$ and $\eta^{*} M$ are the pushouts of $\mathrm{GL}_{1}(A) \rightarrow M$ along $\mathrm{GL}_{1}(D)$ and $\mathrm{GL}_{1}(\eta): \mathrm{GL}_{1}(A) \rightarrow \mathrm{GL}_{1}(A \oplus J)$, respectively. Here $\mathrm{GL}_{1}(A \oplus J)$ is the coproduct of $\mathrm{GL}_{1}(A)$ and $(1+J, \cdot)$ both along $\mathrm{GL}_{1}(D)$ and along $\mathrm{GL}_{1}(\eta)$, so both $D^{*} M$ and $\eta^{*} M$ are coproducts of $M$ and $(1+J, \cdot)$, and $D^{b}$ induces the canonical isomorphism between them.

To corepresent log derivations by a module of log differentials, we express the group of $\log$ derivations as a pullback of the groups of ring derivations and monoid derivations, subject to a compatibility condition. This uses the following definition. 
Definition 4.17 Let $M$ be a commutative monoid, and $K$ an abelian group. The commutative monoid derivations of $M$ with values in $K$ is the abelian group

$$
\operatorname{Der}^{\mathrm{b}}(M, K)=(\mathcal{C} \text { Mon } / M)(M, M \times K)
$$

of monoid homomorphisms $D^{\mathrm{b}}: M \rightarrow M \times K$ over $M$. These all have the form $D^{\mathrm{b}}(m)=\left(m, d^{\mathrm{b}}(m)\right)$, where $d^{\mathrm{b}}: M \rightarrow K$ is a monoid homomorphism, and correspond bijectively to the group homomorphisms $M^{\mathrm{gp}} \rightarrow K$, where $M^{\mathrm{gp}}$ is the group completion of $M$. We might call the abelian group $M^{\text {gp }}$ the commutative monoid differentials of $M$. Recall that we write $\gamma: M \rightarrow M^{\mathrm{gp}}$ for the canonical monoid homomorphism.

Lemma 4.18 The universal monoid derivation $D^{\mathrm{b}}: M \rightarrow M \times M^{\mathrm{gp}}$, taking $m$ to $D^{b}(m)=(m, \gamma(m))$, induces a natural isomorphism

$$
\mathcal{A} b\left(M^{\mathrm{gp}}, K\right) \cong \operatorname{Der}^{\mathrm{b}}(M, K) .
$$

Remark 4.19 In other words, $M^{\text {gp }}$ corepresents commutative monoid derivations. Unlike in the commutative ring case, this construction is already derived, since if $F_{\bullet} \stackrel{\simeq}{\longrightarrow} M$ is a cofibrant simplicial commutative monoid resolution (with $F_{\bullet}$ a free commutative monoid in each simplicial degree), then the degreewise group completion $F_{\bullet}^{\mathrm{gp}}$ is weakly equivalent to $M^{\mathrm{gp}}$. For a proof, see Puppe [58, Section 3.6, Satz 13] or Quillen's appendix in Friedlander-Mazur [24]. In other words, the commutative monoid cotangent complex $\mathbb{L} M^{\mathrm{gp}}=F_{\bullet}^{\mathrm{gp}}$ is weakly equivalent to the commutative monoid differentials $M^{\text {gp }}$.

Proposition 4.20 Let $(A, M, \alpha)$ be a $\log$ ring and $J$ an $A$-module. There is a pullback square

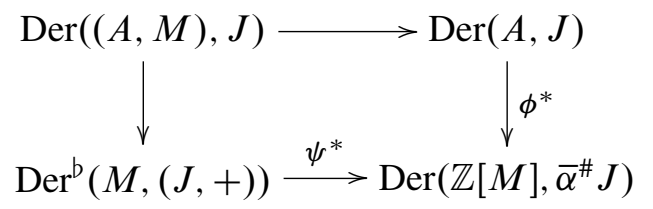

of abelian groups. Here $(J,+)$ denotes the underlying abelian group of $J$, and $\bar{\alpha}^{\#} J$ denotes $J$ viewed as a $\mathbb{Z}[M]$-module via the adjoint log structure map $\bar{\alpha}: \mathbb{Z}[M] \rightarrow A$.

The homomorphism $\phi^{*}$ is induced by the ring homomorphism $\bar{\alpha}$, taking a derivation $D: A \rightarrow A \oplus J$ to the composite $D \circ \bar{\alpha}$. The homomorphism $\psi^{*}$ is induced by the monoid homomorphism $\eta^{*} \alpha: \eta^{*} M \rightarrow(A \oplus J, \cdot)$, taking a monoid derivation $D^{b}: M \rightarrow \eta^{*} M$ to the ring homomorphism $\mathbb{Z}[M] \rightarrow A \oplus J$ that is left adjoint to the composite monoid homomorphism $\eta^{*} \alpha \circ D^{b}$. 
Proof Recall from Lemma 4.5 the identification of $\operatorname{Der}\left(\mathbb{Z}[M], \bar{\alpha}^{\#} J\right)$ with the ring homomorphisms $\mathbb{Z}[M] \rightarrow A \oplus J$ over $A$, and from Lemma 4.12 the identification of $\operatorname{Der}^{\mathrm{b}}(M,(J,+))$ with the monoid homomorphisms $M \rightarrow \eta^{*} M$ over $M$. A $\log$ derivation $\left(D, D^{b}\right):(A, M) \rightarrow\left(A \oplus J, \eta^{*} M\right)$ consists of a ring derivation $D: A \rightarrow$ $A \oplus J$ and a monoid derivation $D^{\mathrm{b}}: M \rightarrow \eta^{*} M$, subject to the compatibility condition that the diagram

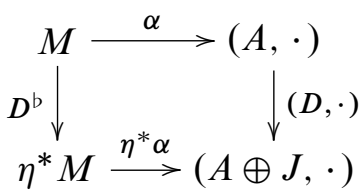

of commutative monoids commutes. By adjunction, this is equivalent to the commutativity of the diagram

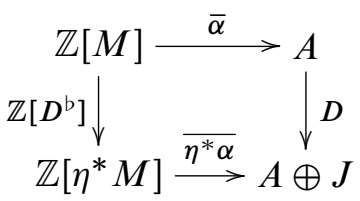

of commutative rings. Hence the pair $\left(D, D^{b}\right)$ defines a derivation homomorphism precisely when $\phi^{*}(D)=\psi^{*}\left(D^{b}\right)$.

Lemma 4.21 Let $(A, M, \alpha)$ be a $\log$ ring. The functors from $A$-modules to abelian groups that take $J$ to $\operatorname{Der}(A, J)$ and $\operatorname{Der}\left(\mathbb{Z}[M], \bar{\alpha}^{\#} J\right)$ are corepresented by the Kähler differentials $\Omega_{A}^{1}$ and the induced $A$-module $A \otimes_{\mathbb{Z}[M]} \Omega_{\mathbb{Z}[M]}^{1}$, respectively. The natural homomorphism $\phi^{*}$ is corepresented by the $A$-module homomorphism

$$
\phi: A \otimes_{\mathbb{Z}[M]} \Omega_{\mathbb{Z}[M]}^{1} \rightarrow \Omega_{A}^{1}
$$

given by

$$
\phi(a \otimes d m)=a \cdot d \alpha(m)
$$

for $a \in A$ and $m \in M$. It is left adjoint to the $\mathbb{Z}[M]$-module homomorphism $\Omega_{\mathbb{Z}[M]}^{1} \rightarrow$ $\Omega_{A}^{1}$ induced by $\bar{\alpha}: \mathbb{Z}[M] \rightarrow A$.

Proof This is clear.

Lemma 4.22 Let $(A, M, \alpha)$ be a log ring. The functors from $A$-modules to abelian groups that take $J$ to $\operatorname{Der}^{b}(M,(J,+))$ and $\operatorname{Der}\left(\mathbb{Z}[M], \bar{\alpha}^{\#} J\right)$ are corepresented by the induced $A$-modules $A \otimes M^{\mathrm{gp}}$ and $A \otimes_{\mathbb{Z}[M]} \Omega_{\mathbb{Z}[M]}^{1}$, respectively, The natural homomorphism $\psi^{*}$ is corepresented by the $A$-module homomorphism

given by

$$
\begin{gathered}
\psi: A \otimes_{\mathbb{Z}[M]} \Omega_{\mathbb{Z}[M]}^{1} \rightarrow A \otimes M^{\mathrm{gp}} \\
\psi(a \otimes d m)=a \cdot \alpha(m) \otimes \gamma(m)
\end{gathered}
$$

for $a \in A$ and $m \in M$. 
Proof For each $A$-module $J$ there are natural chains of isomorphisms

$$
\begin{aligned}
\operatorname{Hom}_{A}\left(A \otimes M^{\mathrm{gp}}, J\right) & \cong \mathcal{A} b\left(M^{\mathrm{gp}},(J,+)\right) \\
& \cong \mathcal{C} \mathcal{M} o n(M,(J,+)) \\
& \cong \operatorname{Der}^{\mathrm{b}}(M,(J,+)) \\
& =(\mathcal{C} \mathcal{M} \text { on } / M)\left(M, \eta^{*} M\right)
\end{aligned}
$$

(using the identification $\eta^{*} M \cong M \times(J,+)$ from Lemma 4.12) and

$$
\begin{aligned}
(\mathcal{C M o n} /(A, \cdot))(M,(A \oplus J, \cdot)) & \cong(\mathcal{C R i n g} / A)(\mathbb{Z}[M], A \oplus J) \\
& \cong \operatorname{Der}\left(\mathbb{Z}[M], \bar{\alpha}^{\#} J\right) \\
& =(\mathcal{C} \mathcal{R i n g} / \mathbb{Z}[M])(\mathbb{Z}[M], \mathbb{Z}[M] \oplus J) \\
& \cong \operatorname{Hom}_{\mathbb{Z}[M]}\left(\Omega_{\mathbb{Z}[M]}^{1}, J\right) \\
& \cong \operatorname{Hom}_{A}\left(A \otimes_{\mathbb{Z}[M]} \Omega_{\mathbb{Z}[M]}^{1}, J\right) .
\end{aligned}
$$

To find the corepresenting homomorphism $\psi$, we let $J=A \otimes M^{\mathrm{gp}}$ and note that the identity homomorphism of $A \otimes M^{\mathrm{gp}}$ corresponds, under the first chain of isomorphisms above, to the monoid homomorphism $D^{\mathrm{b}}: M \rightarrow \eta^{*} M \cong M \times(J,+)$ over $M$ that takes $m$ to $D^{b}(m)=(m, 1 \otimes \gamma(m))$. By Proposition 4.20, $\psi^{*}$ takes this $D^{b}$ to the monoid homomorphism $\eta^{*} \alpha \circ D^{\mathrm{b}}: M \rightarrow\left(A \oplus\left(A \otimes M^{\mathrm{gp}}\right), \cdot\right)$ over $(A, \cdot)$ that takes $m$ to

$$
\alpha(m) \cdot(1 \oplus(1 \otimes \gamma(m)))=\alpha(m) \oplus(\alpha(m) \otimes \gamma(m)) .
$$

Under the second chain of isomorphisms above, this corresponds to the $A$-module homomorphism $\psi: A \otimes_{\mathbb{Z}[M]} \Omega_{\mathbb{Z}[M]}^{1} \rightarrow A \otimes M^{\text {gp }}$ that takes $a \otimes d m$ to $a \cdot \alpha(m) \otimes \gamma(m)$, for $m \in M$. Hence this $\psi$ is the $A$-module homomorphism that corepresents $\psi^{*}$.

Remark 4.23 The elements $m \in M$ generate $\mathbb{Z}[M]$ as a ring, so the $d m$ for $m \in M$ generate $\Omega_{\mathbb{Z}[M]}^{1}$ as a $\mathbb{Z}[M]$-module, and the formula $\psi(1 \otimes d m)=\alpha(m) \otimes \gamma(m)$ determines the $A$-module homomorphism $\psi$. To see that it is well defined, we may check that $\psi(1 \otimes d(m n))=\alpha(m n) \otimes \gamma(m n)$ equals $\psi(1 \otimes((d m) n+m(d n)))=$ $\alpha(m) \alpha(n) \otimes \gamma(m)+\alpha(m) \alpha(n) \otimes \gamma(n)$.

Remark 4.24 The $A$-module homomorphism $\psi$ is induced by the $\mathbb{Z}[M]$-module homomorphism $\Omega_{\mathbb{Z}[M]}^{1} \rightarrow \mathbb{Z}[M] \otimes M^{\text {gp }}$ that takes $d m$ to $\zeta(m) \otimes \gamma(m)$, in the notation of Definitions 2.12 and 3.1. As we shall explain in Remark 13.7, the latter homomorphism corresponds to the stabilization of the repletion map $\psi: \mathbb{Z}[M] \otimes \mathbb{Z}[M] \rightarrow$ $\mathbb{Z}[M] \otimes \otimes^{\text {rep }} \mathbb{Z}[M] \cong \mathbb{Z}[M] \otimes \mathbb{Z}\left[M^{\mathrm{gp}}\right]$, in the stable category associated to the based category $\mathbb{Z}[M] / \mathcal{C} \mathcal{R}$ ing $/ \mathbb{Z}[M]$. 
Definition 4.25 Let $(A, M, \alpha)$ be a pre-log ring. The log Kähler differentials of $(A, M)$ is the $A$-module $\Omega_{(A, M)}^{1}$ defined by the pushout square

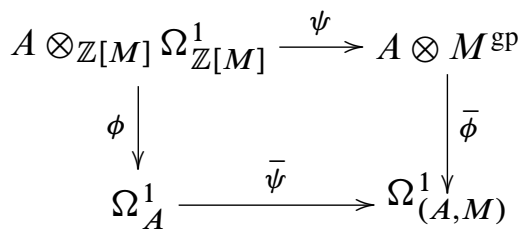

in $A$-modules of the homomorphisms $\phi$ and $\psi$ from Lemmas 4.21 and 4.22 , respectively. We write $d a$ for $\bar{\psi}(d a)$ and $d \log m$ for $\bar{\phi}(1 \otimes \gamma(m))$, with $a \in A$ and $m \in M$, where $\bar{\phi}$ and $\bar{\psi}$ are the canonical homomorphisms. Note that $d(a b)=$ $(d a) b+a(d b), d \log (m n)=d \log m+d \log n$, and $d \alpha(m)=\alpha(m) d \log m$, for $a, b \in A$ and $m, n \in M$.

Remark 4.26 In other words,

$$
\Omega_{(A, M)}^{1}=\Omega_{A}^{1} \oplus\left(A \otimes M^{\mathrm{gp}}\right) / \sim
$$

where $\sim$ is $A$-linearly generated by the relation $d \alpha(m)=\phi(1 \otimes d m) \sim \psi(1 \otimes d m)=$ $\alpha(m) \otimes \gamma(m)$ for $m \in M$. Thus we recover Kato's definition of log differentials [35, (1.7)]. The relation $d \alpha(m)=\alpha(m) d \log m$, which shows that $d \log m$ has the formal properties of the logarithmic differential $a^{-1} d a$ for $a=\alpha(m)$, is the main reason for the use of the adjective "log", or "logarithmic", in this theory.

Like in Kato's introduction, we permit $(M, \alpha)$ to be a pre-log structure in the definition of $\Omega_{(A, M)}^{1}$. If $(M, \alpha)$ (or its logification $\left.(M, \alpha)^{a}\right)$ is the trivial log structure, $\psi$ and $\bar{\psi}$ are isomorphisms, so $\Omega_{(A, M)}^{1} \cong \Omega_{A}^{1}$. See also Lemma 11.27.

Proposition 4.27 Let $(A, M, \alpha)$ be a log ring. The universal log derivation

$$
\left(D, D^{b}\right):(A, M) \rightarrow\left(A \oplus \Omega_{(A, M)}^{1}, \eta^{*} M\right),
$$

taking $a \in A$ to $D(a)=(a, d a)$, and taking $m \in M$ to $D^{\mathrm{b}}(m)=(m, d \log m)$, induces a natural isomorphism

$$
\operatorname{Hom}_{A}\left(\Omega_{(A, M)}^{1}, J\right) \cong \operatorname{Der}((A, M), J) .
$$

Proof Use Proposition 4.20, Lemmas 4.21 and 4.22, and Definition 4.25.

Lemma 4.28 Let $X \subset X \sqcup Y$ be a pair of sets, let $M=\langle X\rangle$ be the free commutative monoid generated by $X$, and let $A=\mathbb{Z}[\langle X \sqcup Y\rangle]$ be the free commutative ring generated by $X \sqcup Y$. Let $\alpha: M \rightarrow(A, \cdot)$ be the monoid homomorphism extending 
the composite inclusion $X \subset X \sqcup Y \rightarrow A$. Then $M^{\mathrm{gp}} \cong \mathbb{Z}\{X\}$ is the free abelian group generated by $X, \phi: A \otimes \mathbb{Z}\{X\} \rightarrow A \otimes \mathbb{Z}\{X \sqcup Y\}$ is the inclusion induced by $X \subset X \sqcup Y, \psi: A \otimes \mathbb{Z}\{X\} \rightarrow A \otimes \mathbb{Z}\{X\}$ is the sum over $x \in X$ of the injective $A$-module homomorphisms $x \cdot A \rightarrow A$ taking $a$ to $x a$, and

$$
\Omega_{(A, M)}^{1} \cong A\{X\} \oplus A\{Y\}
$$

where $x \in X$ and $y \in Y$ correspond to $d \log x$ and $d y$ in $\Omega_{(A, M)}^{1}$, respectively. There is a short exact sequence

$$
0 \rightarrow \Omega_{A}^{1} \stackrel{\bar{\psi}}{\rightarrow} \Omega_{(A, M)}^{1} \stackrel{\text { res }}{\longrightarrow} \bigoplus_{x \in X} A / x A \rightarrow 0,
$$

where the residue map res takes $d \log x$ to $1 \in A / x A$, and $d y$ to 0 .

Remark 4.29 Following Ofer Gabber (see Olsson [55, Section 8]), we can define the $\log$ cotangent complex of a pre-log ring $(A, M)$ as the simplicial $A$-module

$$
\mathbb{L} \Omega_{(A, M)}^{1}=A \otimes_{\left(P_{\bullet}, F_{\bullet}\right)} \Omega_{\left(P_{\bullet}, F_{\bullet}\right)}^{1},
$$

where $\left(P_{\bullet}, F_{\bullet}\right) \stackrel{\simeq}{\longrightarrow}(A, M)$ is a cofibrant simplicial pre-log ring resolution of $(A, M)$. Cofibrancy ensures that in each simplicial degree $q$, the pre-log ring $\left(P_{q}, F_{q}\right)$ is freely generated by a pair of sets $X_{q} \subset X_{q} \sqcup Y_{q}$, as in the lemma above. Our log topological André-Quillen homology spectrum $\operatorname{TAQ}(A, M)$ will be the generalization to pre-log $S$-algebras of this log cotangent complex.

Remark 4.30 For a map $(R, P, \rho) \rightarrow(A, M, \alpha)$ of pre-log rings, Kato also defines an $A$-module $\Omega_{(A, M) /(R, P)}^{1}$ of relative $\log$ Kähler differentials, which agrees with the absolute $\log$ Kähler differentials when $(R, P)=(\mathbb{Z},\{1\})$. The logification maps $(R, P) \rightarrow\left(R, P^{a}\right)$ and $(A, M) \rightarrow\left(A, M^{a}\right)$ induce isomorphisms

$$
\Omega_{(A, M) /(R, P)}^{1} \stackrel{\cong}{\rightarrow} \Omega_{\left(A, M^{a}\right) /(R, P)}^{1} \stackrel{\cong}{\leftrightarrows} \Omega_{\left(A, M^{a}\right) /\left(R, P^{a}\right)}^{1} .
$$

For maps $(R, P, \rho) \rightarrow(A, M, \alpha) \rightarrow(B, N, \beta)$ of fine pre-log rings there is a transitivity exact sequence

$$
B \otimes_{A} \Omega_{(A, M) /(R, P)}^{1} \rightarrow \Omega_{(B, N) /(R, P)}^{1} \rightarrow \Omega_{(B, N) /(A, M)}^{1} \rightarrow 0
$$

of $B$-modules; see Kato [35, Proposition 3.12]. For a pushout square

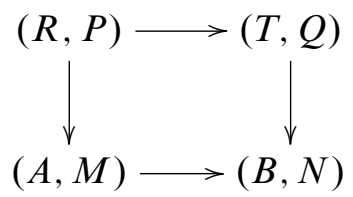


of pre-log rings, with $B=A \otimes_{R} T, N=M \oplus_{P} Q$ and $A$ flat over $R$, there is a base change isomorphism

$$
B \otimes_{T} \Omega_{(T, Q) /(R, P)}^{1} \cong \Omega_{(B, N) /(A, M)}^{1}
$$

of $B$-modules [35, page 196]. We say that $A \rightarrow B$ is formally étale if $\Omega_{B / A}^{1}=0$, and $(A, M) \rightarrow(B, N)$ is formally log étale if $\Omega_{(B, N) /(A, M)}^{1}=0$. We shall discuss topological analogues of these results later in the paper.

Remark 4.31 The log Kähler differentials of the canonical log structure satisfy

$$
\Omega_{(\mathbb{Z}[M], M)}^{1} \cong \mathbb{Z}[M] \otimes M^{\mathrm{gp}} .
$$

Hence, for a general pre-log ring $(A, M)$ there is a pushout square

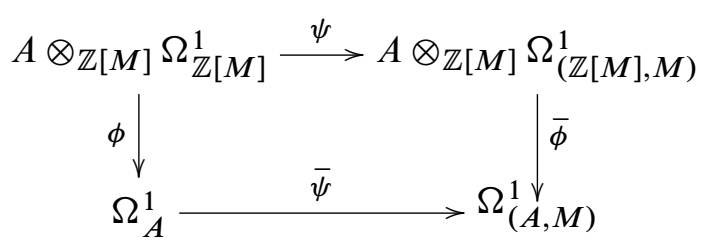

of $A$-modules. The localization map $(A, M) \rightarrow\left(A\left[M^{-1}\right], M^{\mathrm{gp}}\right)$ induces a further map

$$
\Omega_{(A, M)}^{1} \rightarrow \Omega_{\left(A\left[M^{-1}\right], M \text { sp }\right)}^{1} \cong \Omega_{A\left[M^{-1}\right]}^{1},
$$

where the last isomorphism uses that $M^{\mathrm{gp}, a}$ is the trivial log structure on $A\left[M^{-1}\right]$.

Example 4.32 We continue the discussion of log structures on discrete valuation rings from Example 2.16, referring to Serre [75, Section I.6] and Hesselholt-Madsen [29, Section 2.2] for more details. Let $A \rightarrow B$ be a finite extension of discrete valuation rings, with uniformizers $\pi$ and $x$, fraction fields $K \rightarrow L$, and residue fields $k \rightarrow \ell$, respectively. In particular, $B$ is the integral closure of $A$ in $L$. We assume that $K$ and $L$ are of characteristic 0 , and that $k$ and $\ell$ are perfect fields of characteristic $p$.

We can write $\pi=u x^{e}$ in $B$, where $u$ is a unit and $e$ is the ramification index. For simplicity we may assume that $u=1$, since the pre-log structures $\langle\pi\rangle$ and $\left\langle x^{e}\right\rangle$ on $A$ have the same logification. Let $\phi(X) \in A[X]$ be the minimal polynomial of $x$ over $A$, so that $B \cong A[X] /(\phi(X))$. Then

$$
\begin{aligned}
& \Omega_{B / A}^{1} \cong B /\left(\phi^{\prime}(x), e x^{e-1}\right)\{d x\}, \\
& \Omega_{(B,\langle x\rangle) /(A,\langle\pi\rangle)}^{1} \cong B /\left(x \phi^{\prime}(x), e\right)\{d \log x\}
\end{aligned}
$$

since $d \phi(x)=\phi^{\prime}(x) d x, d \pi=d\left(x^{e}\right)=e x^{e-1} d x$ and $d \log \pi=d \log \left(x^{e}\right)=$ e $d \log x$. 
If $A \rightarrow B$ is unramified, so $e=1$, then $\Omega_{B / A}^{1}=0$ and $\Omega_{(B,\langle x\rangle) /(A,\langle\pi\rangle)}^{1}=0$, so $A \rightarrow B$ is (formally) étale and (formally) log étale.

If $A \rightarrow B$ is totally ramified, so $e=[L: K]$, then $\phi(X)$ is an Eisenstein polynomial

$$
\phi(X)=X^{e}-\pi \theta(X)
$$

where $\theta(X) \in A[X]$ has degree $<e$, and $\theta(0)$ is a unit in $A$. Then

$$
\left(\phi^{\prime}(x), e x^{e-1}\right)=\left(\pi \theta^{\prime}(x), e x^{e-1}\right)
$$

is contained in ( $x$ ) unless $e=1$, so $\Omega_{B / A}^{1}=0$ only if $A \rightarrow B$ is an isomorphism. Furthermore,

$$
x \phi^{\prime}(x)=e x^{e}-x \pi \theta^{\prime}(x)=\pi\left(e \theta(x)-x \theta^{\prime}(x)\right)
$$

so $\left(x \phi^{\prime}(x)\right) \subset(\pi) \subset(x)$. Hence $\Omega_{(B,\langle x\rangle) /(A,\langle\pi\rangle)}^{1}=0$ if and only if $e$ is a unit in $B /(x)=\ell$, ie, if and only if $p \nmid e$.

A general finite extension $A \rightarrow B$ is the composite of an unramified and a totally ramified extension, so $A \rightarrow B$ is étale if and only if $e=1$, and $(A,\langle\pi\rangle) \rightarrow(B,\langle x\rangle)$ is $\log$ étale if and only if $p \nmid e$, ie, if and only if $A \rightarrow B$ is tamely ramified. In this way, log geometry extends the range of étaleness (and smoothness) to allow tame ramification.

\section{Symmetric logarithmic structures}

We recall an interpretation of the Hochschild homology of a ring, based on Quillen [60, Section 3], which is similar to the interpretation of the Kähler differentials as a corepresenting object for derivations. Thereafter we extend this point of view to the $\log$ case.

Definition 5.1 Let $A$ be an associative ring, always with unit, and let $A^{e}=A \otimes A^{\text {op }}$, so that $A^{e}$-modules are the same as $A$-bimodules. Let $K$ be an $A$-bimodule. The square-zero extension $A \oplus K$ is the associative ring with multiplication

$$
\left(a_{1} \oplus k_{1}\right) \cdot\left(a_{2} \oplus k_{2}\right)=a_{1} a_{2} \oplus\left(k_{1} a_{2}+a_{1} k_{2}\right) .
$$

The augmentation $\epsilon: A \oplus K \rightarrow A$ taking $a \oplus k$ to $a$ makes $A \oplus K$ an associative ring over $A$, with two-sided augmentation ideal $K$ having the zero multiplication.

Remark 5.2 The structure maps $\eta, \mu$ and $\chi$, defined as in Remark 4.2, make $A \oplus K$ an abelian group object in the category $\mathcal{A R i n g} / A$ of associative rings over $A$. The functor $K \mapsto A \oplus K$ is an equivalence from the category $\mathcal{M o d}_{A^{e}}$ of $A$-bimodules to the category $(\mathcal{A R} \text { ing } / A)_{\mathrm{ab}}$. See Quillen [60, Section 3]. 
Definition 5.3 The associative derivations of $A$ with values in $K$ is the abelian group

$$
\operatorname{ADer}(A, K)=(\mathcal{A R} \operatorname{Ring} / A)(A, A \oplus K)
$$

of associative ring homomorphisms $D: A \rightarrow A \oplus K$ over $A$. Define the $A$-bimodule $D_{A}$ of associative differentials by the short exact sequence

$$
0 \rightarrow D_{A} \stackrel{i}{\rightarrow} A \otimes A \stackrel{m}{\rightarrow} A \rightarrow 0
$$

of $A$-bimodules, where $m(a \otimes b)=a b$. It corepresents associative derivations, in the following sense.

Lemma 5.4 The universal associative derivation $D: A \rightarrow A \oplus D_{A}$, taking a to $D(a)=a \oplus d a$ where $i(d a)=1 \otimes a-a \otimes 1$, induces a natural isomorphism

$$
\operatorname{Hom}_{A^{e}}\left(D_{A}, K\right) \cong \operatorname{ADer}(A, K) .
$$

When $A=T(X)$ is the free associative ring generated by a set $X, \operatorname{ADer}(T(X), K) \cong$ $\{$ functions $X \rightarrow K\}$, so $D_{T(X)} \cong T(X)^{e} \otimes \mathbb{Z}\{X\}$ is a free $T(X)^{e}$-module.

Definition 5.5 If $A$ is a commutative ring, so that $m: A \otimes A \rightarrow A$ is a ring homomorphism, we say that an $A$-bimodule $K$ is symmetric if the left and right $A$-module actions on $K$ agree: $a \cdot k=k \cdot a$ for $a \in A, k \in K$. Equivalently, $K$ is symmetric if $K \cong m^{\#} J$, where $J$ is the underlying left $A$-module of $K$ and $m^{\#} J$ denotes the $A$-bimodule obtained from $J$ by restriction along $m$. Let

$$
\operatorname{SDer}(A, J)=\operatorname{ADer}\left(A, m^{\#} J\right)
$$

be the symmetric derivations of $A$ with values in $J$.

Lemma 5.6 The restriction functor $m^{\#}: \mathcal{M o d}_{A} \rightarrow \mathcal{M o d}_{A}{ }^{e}$ is compatible with the forgetful functor $(\mathcal{C R} i n g / A)_{\mathrm{ab}} \rightarrow(\mathcal{A R} i n g / A)_{\mathrm{ab}}$ under the equivalences $\mathcal{M o d}_{A} \simeq$ $(\mathcal{C R i n g} / A)_{\mathrm{ab}}$ and $\operatorname{Mod}_{A^{e}} \simeq(\mathcal{A R i n g} / A)_{\mathrm{ab}}$.

Proof The forgetful functor between abelian group objects exists because the forgetful functor $\mathcal{C}$ Ring / $A \rightarrow \mathcal{A} \mathcal{R} i n g / A$ preserves finite products. The compatibility amounts to the fact that $A \oplus J$ in Definition 4.1 agrees with $A \oplus m^{\#} J$ in Definition 5.3.

Lemma 5.7 Let $A$ be a commutative ring, let $J$ be an $A$-module, and let $m^{\#} J$ be the corresponding symmetric $A$-bimodule. There is a natural isomorphism

$$
\operatorname{Hom}_{A}\left(A \otimes_{A^{e}} D_{A}, J\right) \cong \operatorname{ADer}\left(A, m^{\#} J\right) .
$$

In other words, the symmetric derivations of $A$ are corepresented by the $A$-module $A \otimes_{A^{e}} D_{A}$ of symmetric differentials. 
Remark 5.8 For a commutative ring $A$ and an $A$-module $J$, a symmetric derivation $D: A \rightarrow A \oplus m^{\#} J$ over $A$ is the same as an ordinary derivation $D: A \rightarrow A \oplus J$ over $A$. Hence the symmetric differentials $A \otimes_{A^{e}} D_{A} \cong D_{A} / D_{A}^{2}$ are canonically isomorphic to the Kähler differentials $\Omega_{A}^{1}$. However, since $D_{A}$ is defined for associative rings and $\Omega_{A}^{1}$ for commutative rings, their homotopically derived functors will be different.

The case of Kähler differentials and the cotangent complex was discussed in Remark 4.7. In the associative setting the functor $A \mapsto \operatorname{ADer}(A, K)$ acquires homotopical right derived functors when extended to simplicial rings. These functors are corepresented by the simplicial $A$-bimodule $\mathbb{L} D_{A}=A^{e} \otimes_{T_{\bullet}^{e}} D_{T_{\bullet}}$, where $T_{\bullet} \stackrel{\simeq}{\longrightarrow} A$ is a cofibrant simplicial ring resolution of $A$. As usual, cofibrant effectively means that $T_{\bullet}$ is a free associative ring, ie, a tensor algebra over $\mathbb{Z}$, in each simplicial degree. We call $\mathbb{L} D_{A}$ the associative cotangent complex. When the composite functor $A \mapsto \operatorname{SDer}(A, J)=$ $\operatorname{ADer}\left(A, m^{\#} J\right)$ is derived in the same way, the corepresenting object is the simplicial $A$-module $A \otimes_{A^{e}} \mathbb{L} D_{A} \cong A \otimes_{T_{\bullet}^{e}} D_{T_{\bullet}}$, which we call the symmetric cotangent complex.

Remark 5.9 By Lemma 5.4, $\mathbb{L} D_{A}$ is a free $A^{e}$-module in each simplicial degree. If we assume that $A$ is flat over $\mathbb{Z}$, then $T_{\bullet}^{e} \stackrel{\simeq}{\longrightarrow} A^{e}$, so $\mathbb{L} D_{A} \stackrel{\simeq}{\longrightarrow} D_{A}$ is a free $A^{e}$ module resolution. Let $B_{\bullet}=B(A, A, A)=\Delta_{\bullet}^{1} \otimes A$ be the two-sided bar construction on $A$. Since we are assuming that $A$ is flat over $\mathbb{Z}, \epsilon: B_{\bullet} \stackrel{\simeq}{\longrightarrow} A$ is a flat $A^{e}$-module resolution, and there is a weak equivalence $A \otimes_{A^{e}} \mathbb{L} D_{A} \simeq B_{\bullet} \otimes_{A^{e}} D_{A}$. Hence the short exact sequence of $A$-bimodules defining $D_{A}$ yields a homotopy cofiber sequence

$$
A \otimes_{A^{e}} \mathbb{L} D_{A} \rightarrow B_{\bullet} \rightarrow \mathrm{HH}(A)
$$

of simplicial $A$-modules, where we use that $B_{\bullet} \otimes_{A^{e}} A \cong \mathrm{HH}(A)$. The left (or right) unit inclusion $A \rightarrow A \otimes A=B_{0} \rightarrow B_{\bullet}$ is a weak equivalence, and the composite map $A \rightarrow \mathrm{HH}(A)$ equals the usual structure map $\eta$. We might therefore, somewhat imprecisely, say that

$$
A \otimes_{A^{e}} \mathbb{L} D_{A} \rightarrow A \stackrel{\eta}{\rightarrow} \mathrm{HH}(A)
$$

is a cofiber sequence up to homotopy, where $\eta$ is split injective. In particular, there are isomorphisms

$$
\mathrm{HH}_{q+1}(A) \cong \pi_{q}\left(A \otimes_{A^{e}} \mathbb{L} D_{A}\right) \cong \operatorname{Tor}_{q}^{A^{e}}\left(A, D_{A}\right)
$$

for $q \geq 0$.

Definition 5.10 Let $A$ be an associative ring. An associative pre-log structure $(M, \alpha)$ on $A$ is an associative monoid $M$ and a monoid homomorphism $\alpha: M \rightarrow(A, \cdot)$ to the underlying multiplicative monoid. It is an associative log structure if the restricted homomorphism $\alpha^{-1} \mathrm{GL}_{1}(A) \rightarrow \mathrm{GL}_{1}(A)$ is an isomorphism. An associative (pre-)log 
ring is an associative ring with an associative (pre-)log structure. A homomorphism $\left(f, f^{b}\right):(A, M) \rightarrow(B, N)$ of associative pre-log rings is a ring homomorphism $f: A \rightarrow B$ and a monoid homomorphism $f^{b}: M \rightarrow N$ such that $(f, \cdot) \circ \alpha=\beta \circ f^{b}$. Associative $\log$ rings generate a full subcategory $\mathcal{A} \log$ of the category $\mathcal{A} \mathcal{P r e} \log$ of associative pre-log rings.

Lemma 5.11 Let $(A, M)$ be a log ring, and let $J$ be an $A$-module. Then $(A, M)$ is an associative $\log$ ring, and the forgetful functor $(\mathcal{L} o g /(A, M))_{\mathrm{ab}} \rightarrow(\mathcal{A} \mathcal{L} \operatorname{og} /(A, M))_{\mathrm{ab}}$ takes the abelian group object $\left(A \oplus J, \eta^{*} M\right)$ in $\mathcal{L} \circ g /(A, M)$ to an abelian group object $\left(A \oplus m^{\#} J, \eta^{*} M\right)$ in $\mathcal{A} \mathcal{L} o g /(A, M)$. Here $\eta^{*} M \cong M \times(J,+)$.

Proof The underlying associative ring of a commutative log ring $(A, M)$ is an associative $\log$ ring, since the forgetful functor preserves the formation of $\mathrm{GL}_{1}(A) \subset(A, \cdot)$ and the pullback $\alpha^{-1} \mathrm{GL}_{1}(A) \subset M$. There is a forgetful functor between abelian group objects because the forgetful functor $\log /(A, M) \rightarrow \mathcal{A} \log /(A, M)$ preserves finite products. The factorization of $\eta^{*} M$ is from Lemma 4.12.

Remark 5.12 We omit to discuss inverse images of associative log structures, general abelian group objects in $\mathcal{A} \mathcal{L} o g /(A, M)$, associative log derivations, and associative $\log$ differentials.

Definition 5.13 Let $(A, M)$ be a (commutative) log ring, and let $J$ be an $A$-module. The log symmetric derivations of $(A, M)$ with values in $J$ is the abelian group

$$
\operatorname{SDer}((A, M), J)=(\mathcal{A L o g} /(A, M))\left((A, M),\left(A \oplus m^{\#} J, \eta^{*} M\right)\right)
$$

of homomorphisms $\left(D, D^{\mathrm{b}}\right):(A, M) \rightarrow\left(A \oplus m^{\#} J, \eta^{*} M\right)$ of associative log rings over $(A, M)$.

Definition 5.14 Let $M$ be an associative monoid, and $K$ an abelian group. The associative monoid derivations of $M$ with values in $K$ is the abelian group

$$
\operatorname{ADer}^{b}(M, K)=(\mathcal{A M o n} / M)(M, M \times K)
$$

of monoid homomorphisms $D^{\mathrm{b}}: M \rightarrow M \times K$ over $M$. Let $H_{1}(B M) \cong M^{\text {ab,gp }}$ be the abelian group of associative monoid differentials of $M$.

If $M$ is commutative, the symmetric monoid derivations of $M$ with values in $K$ is the abelian group

$$
\operatorname{SDer}^{\mathrm{b}}(M, K)=(\mathcal{A M} \text { on } / M)(M, M \times K)
$$

of monoid homomorphisms $D^{\mathrm{b}}: M \rightarrow M \times K$ over $M$. Let $H_{1}(B M) \cong M^{\text {gp }}$ be the symmetric monoid differentials of $M$. 
Lemma 5.15 There is a universal associative monoid derivation

$$
D^{b}: M \rightarrow M \times H_{1}(B M),
$$

taking $m$ to $D^{b}(m)=m \oplus[m]$ where $[m]$ is the homology class of $m$ viewed as a 1 -simplex in $B M$. It induces a natural isomorphism

$$
\mathcal{A} b\left(H_{1}(B M), K\right) \cong \operatorname{ADer}^{b}(A, K) .
$$

When $M$ is a free associative monoid, there is a weak equivalence

$$
\Sigma H_{1}(B M) \simeq \widetilde{\mathbb{Z}}\{B M\}
$$

of simplicial abelian groups, where $\Sigma H_{1}(B M)$ is the simplicial suspension of the constant simplicial abelian group $H_{1}(B M), \mathbb{Z}\{B M\}$ is the degreewise free abelian group on the simplicial set $B M$, and $\widetilde{\mathbb{Z}}\{B M\}$ is the kernel of the augmentation $\mathbb{Z}\{B M\} \rightarrow \mathbb{Z}$.

Proof For each abelian group $K$, there is a natural chain of isomorphisms

$$
\begin{aligned}
(\mathcal{A M o n} / M)(M, M \times K) & \cong \mathcal{A M} \operatorname{Mon}(M, K) \\
& \cong \mathcal{C} \mathcal{M} \operatorname{con}\left(M^{\mathrm{ab}}, K\right) \\
& \cong \mathcal{A} b\left(M^{\mathrm{ab}, \mathrm{gp}}, K\right)=\mathcal{A} b\left(H_{1}(B M), K\right) .
\end{aligned}
$$

When $M$ is the free associative monoid on a set $X, B M$ is weakly equivalent to a wedge sum of $X$ circles, so $\widetilde{\mathbb{Z}}\{B M\}$ has homotopy concentrated in dimension 1 , which makes it weakly equivalent to the suspension $\Sigma H_{1}(B M)$.

Remark 5.16 As for derivations of rings, symmetric monoid derivations and commutative monoid derivations are the same, but their homotopically derived functors are different. The associative monoid cotangent complex of $M$ is the simplicial abelian group $\mathbb{L} H_{1}(B M)=H_{1}\left(B F_{\bullet}\right)$, where $F_{\bullet} \stackrel{\simeq}{\longrightarrow} M$ is a simplicial resolution of $M$ by free associative monoids. By Lemma 5.15 there is a weak equivalence $\Sigma H_{1}\left(B F_{\bullet}\right) \simeq \widetilde{\mathbb{Z}}\left\{B F_{\bullet}\right\}$, and $\widetilde{\mathbb{Z}}\left\{B F_{\bullet}\right\} \simeq \widetilde{\mathbb{Z}}\{B M\}$ by the Hurewicz theorem, so

$$
\Sigma \mathbb{L} H_{1}(B M) \simeq \widetilde{\mathbb{Z}}\{B M\}
$$

has homotopy groups $\pi_{q} \mathbb{L} H_{1}(B M) \cong H_{q+1}(B M)$ for $q \geq 0$, isomorphic to the higher homology groups of $B M$.

When $M$ is a commutative monoid, the symmetric monoid cotangent complex of $M$ is the same simplicial abelian group $\mathbb{L} H_{1}(B M)=H_{1}\left(B F_{\bullet}\right)$, where $F_{\bullet} \stackrel{\simeq}{\longrightarrow} M$ is a cofibrant associative monoid resolution of $M$, so the formula $\Sigma \mathbb{L} H_{1}(B M) \simeq \widetilde{\mathbb{Z}}\{B M\}$ continues to hold. 
Proposition 5.17 Let $(A, M, \alpha)$ be a (commutative) $\log$ ring and $J$ an $A$-module. There is a pullback square

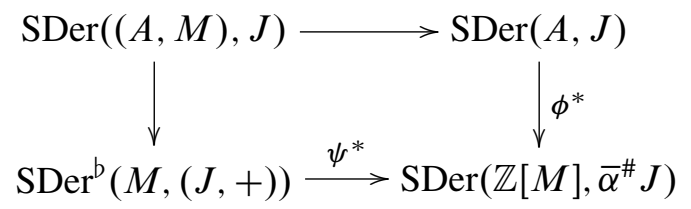

of abelian groups. The homomorphism $\phi^{*}$ is induced by $\bar{\alpha}: \mathbb{Z}[M] \rightarrow A$, and the homomorphism $\psi^{*}$ is induced by $\eta^{*} \alpha: M \times(J,+) \cong \eta^{*} M \rightarrow(A \oplus J, \cdot)$.

Lemma 5.18 The functors from $A$-modules to abelian groups taking $J$ to $\operatorname{SDer}(A, J)$ and $\operatorname{SDer}\left(\mathbb{Z}[M], \bar{\alpha}^{\#} J\right)$ are corepresented by the symmetric differentials $A \otimes_{A^{e}} D_{A}$ and the induced $A$-module $A \otimes_{\mathbb{Z}[M]^{e}} D_{\mathbb{Z}[M]}$, respectively. The natural homomorphism $\phi^{*}$ is corepresented by the $A$-module homomorphism

$$
\phi: A \otimes_{\mathbb{Z}[M]^{e}} D_{\mathbb{Z}[M]} \rightarrow A \otimes_{A^{e}} D_{A}
$$

induced by $\bar{\alpha}: \mathbb{Z}[M] \rightarrow A$.

Lemma 5.19 The functors from $A$-modules to abelian groups that take $J$ to

$$
\operatorname{SDer}^{b}(M,(J,+)) \quad \text { and } \quad \operatorname{SDer}\left(\mathbb{Z}[M], \bar{\alpha}^{\#} J\right)
$$

are corepresented by $A \otimes M^{\text {gp }}$ and $A \otimes_{\mathbb{Z}[M]^{e}} D_{\mathbb{Z}[M]}$, respectively. The natural homomorphism $\psi^{*}$ is corepresented by the $A$-module homomorphism

given by

$$
\begin{gathered}
\psi: A \otimes_{\mathbb{Z}[M]^{e}} D_{\mathbb{Z}[M]} \rightarrow A \otimes M^{\mathrm{gp}} \\
\psi(a \otimes d m)=a \cdot \alpha(m) \otimes \gamma(m)
\end{gathered}
$$

for $a \in A$ and $m \in M$.

Proof We have a natural chain of isomorphisms

$$
\begin{aligned}
(\mathcal{A M o n} /(A, \cdot))(M,(A \oplus J, \cdot)) & \cong(\mathcal{A R i n g} / A)(\mathbb{Z}[M], A \oplus J) \\
& \cong \operatorname{ADer}\left(\mathbb{Z}[M], \bar{\alpha}^{\#} J\right) \\
& =(\mathcal{A} \mathcal{R i n g} / A)(\mathbb{Z}[M], \mathbb{Z}[M] \oplus J) \\
& \cong \mathcal{M o d}_{\mathbb{Z}[M]^{e}}\left(D_{\mathbb{Z}[M]}, J\right) \\
& \cong \mathcal{M o d}_{A}\left(A \otimes_{\mathbb{Z}[M]^{e}} D_{\mathbb{Z}[M]}, J\right) .
\end{aligned}
$$

Let $J=A \otimes M^{\mathrm{gp}}$ and note, as in the proof of Lemma 4.22, that the identity homomorphism of $A \otimes M^{\text {gp }}$ corresponds to a $D^{\mathrm{b}} \in \operatorname{SDer}^{\mathrm{b}}(M,(J,+))$ that maps 
under $\psi^{*}$ to the monoid homomorphism $\eta^{*} \alpha \circ D^{b}$ over $(A, \cdot)$ that takes $m$ in $M$ to $\alpha(m) \oplus(\alpha(m) \otimes \gamma(m))$ in $(A \oplus J, \cdot)$. Under the chain of isomorphisms above, this corresponds to the $\mathbb{Z}[M]$-bimodule homomorphism that takes $d m$ in $D_{\mathbb{Z}[M]}$ to $\alpha(m) \otimes \gamma(m)$ in $J$, and thus to the asserted $A$-module homomorphism.

Definition 5.20 Let $(A, M, \alpha)$ be a (commutative) pre-log ring. The symmetric log Kähler differentials of $(A, M)$ is the $A$-module $A \otimes_{A^{e}} D_{(A, M)}$ defined by the pushout square

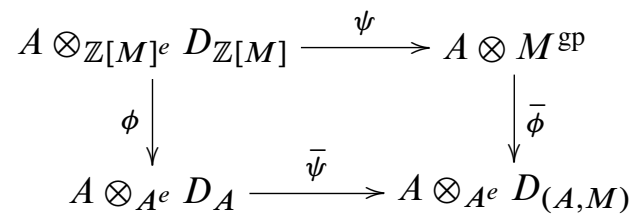

in $A$-modules.

Proposition 5.21 There is a natural isomorphism

$$
\operatorname{Hom}_{A}\left(A \otimes_{A^{e}} D_{(A, M)}, J\right) \cong \operatorname{SDer}((A, M), J) .
$$

Remark 5.22 The symmetric log cotangent complex should now be constructed as

$$
\mathbb{L}\left(A \otimes_{A^{e}} D_{(A, M)}\right)=A \otimes_{T_{\bullet}^{e}} D_{\left(T_{\bullet}, F_{\bullet}\right)}
$$

using a cofibrant replacement $\left(T_{\bullet}, F_{\bullet}\right) \stackrel{\simeq}{\longrightarrow}(A, M)$ in a (closed) model structure on simplicial associative log rings, in the sense of Quillen [59]. We have not worked out the details of such a model structure, but it is clear that the simplicial object above will be the pushout in a suitable category of the maps

$$
A \otimes \mathbb{L} H_{1}(B M) \stackrel{\mathbb{L} \psi}{\longleftarrow} A \otimes_{\mathbb{Z}[M]^{e}} \mathbb{L} D_{\mathbb{Z}[M]} \stackrel{\mathbb{L} \phi}{\longrightarrow} A \otimes_{A^{e}} \mathbb{L} D_{A}
$$

connecting the symmetric cotangent complex of $\mathbb{Z}[M]$ to the symmetric monoid cotangent complex of $M$ and the symmetric cotangent complex of $A$.

Recall the cofiber sequence up to homotopy

$$
A \otimes_{A^{e}} \mathbb{L} D_{A} \rightarrow A \stackrel{\eta}{\rightarrow} \mathrm{HH}(A)
$$

from Remark 5.9, where $\eta$ is split injective as a map of simplicial commutative rings. The analogous sequence for $\mathbb{Z}[M]$ takes the form

$$
A \otimes_{\mathbb{Z}[M]^{e}} \mathbb{L} D_{\mathbb{Z}[M]} \rightarrow A \stackrel{\eta}{\rightarrow} A \otimes_{\mathbb{Z}[M]} \mathrm{HH}(\mathbb{Z}[M]),
$$


after base change along $\bar{\alpha}: \mathbb{Z}[M] \rightarrow A$. By Lemma 5.15 there is also a cofiber sequence up to homotopy

$$
A \otimes \mathbb{L} H_{1}(B M) \rightarrow A \stackrel{\eta}{\rightarrow} A \otimes \mathbb{Z}[B M],
$$

where $\eta$ is again split injective, with mapping cone $A \otimes \Sigma \mathbb{L} H_{1}(B M) \simeq A \otimes \widetilde{\mathbb{Z}}\{B M\}$. Here $\mathbb{Z}[B M]=\mathbb{Z}\{B M\}$ as simplicial abelian groups, but since $B M$ is a simplicial commutative monoid, we can also think of $\mathbb{Z}[B M]$ as a simplicial commutative ring, and therefore we switch to the monoid ring notation.

This suggests that the log Hochschild homology of $(A, M)$ should sit in a cofiber sequence up to homotopy

$$
\mathbb{L}\left(A \otimes_{A^{e}} D_{(A, M)}\right) \rightarrow A \stackrel{\eta}{\rightarrow} \mathrm{HH}(A, M)
$$

with $\eta$ split injective. In particular, $\operatorname{HH}(A, M)$ should be a homotopy pushout of homomorphisms

$$
A \otimes \mathbb{Z}[B M] \stackrel{\psi}{\leftarrow} A \otimes_{\mathbb{Z}[M]} \mathrm{HH}(\mathbb{Z}[M]) \stackrel{\phi}{\rightarrow} \mathrm{HH}(A)
$$

in a suitable category.

Lemma 5.23 The extension $\phi: A \otimes_{\mathbb{Z}[M]} \mathrm{HH}(\mathbb{Z}[M]) \rightarrow \mathrm{HH}(A)$ of (the suspension of) $\mathbb{L} \phi: A \otimes_{\mathbb{Z}[M]^{e}} \mathbb{L} D_{\mathbb{Z}[M]} \rightarrow A \otimes_{A^{e}} \mathbb{L} D_{A}$ is homotopy equivalent to the natural homomorphism of simplicial commutative rings induced by $\bar{\alpha}: \mathbb{Z}[M] \rightarrow A$. It is given by

$$
\phi\left(a \otimes\left(m_{0}, m_{1}, \ldots, m_{q}\right)\right)=\left(a \alpha\left(m_{0}\right), \alpha\left(m_{1}\right), \ldots, \alpha\left(m_{q}\right)\right)
$$

in simplicial degree $q$.

Lemma 5.24 The extension $\psi: A \otimes_{\mathbb{Z}[M]} \mathrm{HH}(\mathbb{Z}[M]) \rightarrow A \otimes \mathbb{Z}[B M]$ of (the suspension of) $\mathbb{L} \psi: A \otimes_{\mathbb{Z}[M]^{e}} \mathbb{L} D_{\mathbb{Z}[M]} \rightarrow A \otimes \mathbb{L} H_{1}(B M)$ is homotopy equivalent to the natural homomorphism of simplicial commutative rings obtained from

$$
\mathbb{Z}[(\epsilon, \pi)]: \operatorname{HH}(\mathbb{Z}[M]) \cong \mathbb{Z}\left[B^{\mathrm{cy}} M\right] \rightarrow \mathbb{Z}[M \times B M] \cong \mathbb{Z}[M] \otimes \mathbb{Z}[B M]
$$

by base change along $\bar{\alpha}: \mathbb{Z}[M] \rightarrow A$. Here $\epsilon: B^{\text {cy }} M \rightarrow M$ and $\pi: B^{\text {cy }} M \rightarrow B M$ are the natural maps taking $\left(m_{0}, m_{1}, \ldots, m_{q}\right)$ in simplicial degree $q$ to $\prod_{i=0}^{q} m_{i}$ and $\left[m_{1}|\cdots| m_{q}\right]$, respectively. The simplicial ring homomorphism is given by

$$
\psi\left(a \otimes\left(m_{0}, m_{1}, \ldots, m_{q}\right)\right)=a \prod_{i=0}^{q} \alpha\left(m_{i}\right) \otimes\left[m_{1}|\cdots| m_{q}\right]
$$

in simplicial degree $q$. 
Proof By Lemma 5.19, the extension $\psi: A \otimes_{\mathbb{Z}[M]^{e}} D_{\mathbb{Z}[M]} \rightarrow A \otimes M^{\text {gp }}$ takes $1 \otimes d m$ to $\alpha(m) \otimes \gamma(m)$. The identification $A \otimes_{\mathbb{Z}[M]^{e}} D_{\mathbb{Z}[M]} \cong A \otimes_{\mathbb{Z}[M]} \mathrm{HH}_{1}(\mathbb{Z}[M])$ takes $1 \otimes d m$ to $1 \otimes(1, m)=1 \otimes \sigma m$, and the identification $A \otimes M^{\mathrm{gp}} \cong A \otimes H_{1}(B M)$ takes $\alpha(m) \otimes \gamma(m)$ to $\alpha(m) \otimes[m]$. Hence $\psi: A \otimes_{\mathbb{Z}[M]} \mathrm{HH}(\mathbb{Z}[M]) \rightarrow A \otimes \mathbb{Z}[B M]$ takes $1 \otimes \sigma m$ to $\alpha(m) \otimes[m]$, and agrees with the claimed formula in dimensions $\leq 1$.

When $M$ is an associative monoid, we interpret $A \otimes_{\mathbb{Z}[M]} \mathrm{HH}(\mathbb{Z}[M])$ as $\mathrm{HH}(\mathbb{Z}[M], A)$, ie, the Hochschild homology of $\mathbb{Z}[M]$ with coefficients in the bimodule $A$. When $M$ is free associative, both $\mathrm{HH}(\mathbb{Z}[M], A)$ and $A \otimes \mathbb{Z}[B M]$ are trivial in dimensions $\geq 2$, hence $\psi$ agrees with the claimed formula in all dimensions.

Returning to the case of a commutative monoid $M$, let $F_{\bullet} \stackrel{\simeq}{\longrightarrow} M$ be a resolution by a simplicial associative monoid that is free associative in each degree. Then $\mathrm{HH}\left(\mathbb{Z}\left[F_{\bullet}\right], A\right) \rightarrow A \otimes \mathbb{Z}\left[B F_{\bullet}\right]$ agrees with the claimed formula for $\psi$ in all degrees and dimensions. It follows (modulo coherence) that $\psi: A \otimes_{\mathbb{Z}[M]} \operatorname{HH}(\mathbb{Z}[M]) \cong$ $\mathrm{HH}(\mathbb{Z}[M], A) \rightarrow A \otimes \mathbb{Z}[B M]$ is given by the asserted formula, since both maps $\mathrm{HH}\left(\mathbb{Z}\left[F_{\bullet}\right], A\right) \rightarrow \operatorname{HH}(\mathbb{Z}[M], A)$ and $\mathbb{Z}\left[B F_{\bullet}\right] \rightarrow \mathbb{Z}[B M]$ are weak equivalences.

Remark 5.25 Given Lemmas 5.23 and 5.24, it is quite clear that $\operatorname{HH}(A, M)$ should be the pushout of $\phi$ and $\psi$ in the category of simplicial commutative rings, so that we have the following three (homotopy) pushout squares

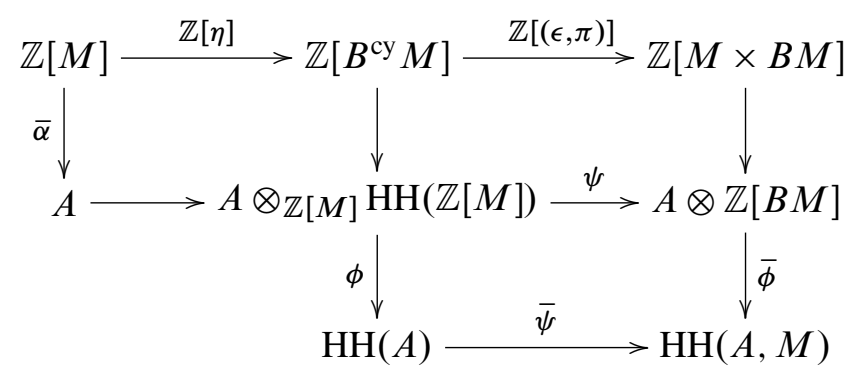

in that category. Up to the weak equivalence $M \times B M \stackrel{\simeq}{\longrightarrow} M \times B M^{\mathrm{gp}} \cong B^{\text {rep }} M$ of Lemma 3.17, the composite of the two right hand squares is exactly the same as the second pushout square of Definition 3.23, where $\operatorname{HH}(A, M)$ was defined as the replete tensor product of $S_{\bullet}^{1}$ copies of $(A, M)$. We view this agreement of constructions, one in terms of replete pre-log structures, and the other in terms of symmetric log derivations, as a confirmation that both notions are meaningful and appropriate. However, the former definition has the advantage that it produces a cyclic object, and the structure maps $\phi$ and $\psi$ defining the pushout are not just defined up to homotopy. These features will be essential when we proceed to consider cyclotomic structure in the topological context. 
Remark 5.26 One may reverse engineer the passage between the symmetric log cotangent complex and the log Hochschild homology, to determine that the morphisms $\mathbb{L} \phi$ and $\mathbb{L} \psi$ in Remark 5.22 should be viewed as morphisms in a category of desuspended simplicial nonunital commutative rings, and the pushout defining $\mathbb{L}\left(A \otimes_{A^{e}} D_{(A, M)}\right)$ should be formed in that category. In other words, the suspension $\Sigma \mathbb{L}\left(A \otimes_{A^{e}} D_{(A, M)}\right)$ is the pushout of $\Sigma \mathbb{L} \phi$ and $\Sigma \mathbb{L} \psi$ in simplicial nonunital commutative rings. This fits with the degree zero part $A \otimes_{A^{e}} D_{(A, M)}$ being the pushout of $\phi$ and $\psi$ in $A$-modules, as in Definition 5.20.

Definition 5.27 Let $R \rightarrow A$ be a homomorphism of commutative rings. The de Rham complex

$$
\Omega_{A / R}^{*}=\Lambda_{A}^{*} \Omega_{A / R}^{1}
$$

is the exterior algebra over $A$ on the Kähler differentials of $A$ relative to $R$. It is the free graded commutative $A$-algebra generated by the $A$-module $\Omega_{A / R}^{1}$. When $R=\mathbb{Z}$ we omit it from the notation. As in Remark 5.8 there are identifications

$$
\Omega_{A / R}^{1} \cong A \otimes_{A^{e}} D_{A / R} \cong \mathrm{HH}_{1}^{R}(A),
$$

taking $a d b$ to the Hochschild class of $a \otimes b$. In view of the graded commutative $A$-algebra structure on $\mathrm{HH}_{*}^{R}(A)$ there is a canonical map

$$
\Omega_{A / R}^{*} \rightarrow \mathrm{HH}_{*}^{R}(A) .
$$

By the Hochschild-Kostant-Rosenberg theorem [31] (see also Loday [42, 3.4.4]), this map is an isomorphism when $A$ is smooth over $R$.

Let $(R, P) \rightarrow(A, M)$ be a homomorphism of pre-log rings. The log de Rham complex

$$
\Omega_{(A, M) /(R, P)}^{*}=\Lambda_{A}^{*} \Omega_{(A, M) /(R, P)}^{1}
$$

is the exterior algebra over $A$ on the log Kähler differentials of $(A, M)$ relative to $(R, P)$. It is the free graded commutative $A$-algebra generated by $\Omega_{(A, M) /(R, P)}^{1}$. When $(R, P)=(\mathbb{Z},\{1\})$ (the absolute case) we omit it from the notation. There are identifications

$$
\Omega_{(A, M) /(R, P)}^{1} \cong A \otimes_{A^{e}} D_{(A, M) /(R, P)} \cong \mathrm{HH}_{1}^{(R, P)}(A, M)
$$

taking $a d b$ and $a d \log m$ to the log Hochschild classes of $\bar{\psi}(a \otimes b)$ and $\bar{\phi}(a \otimes[m])$, respectively. See Remark 3.24 and Definitions 4.25 and 5.20. Hence there is a canonical map

$$
\Omega_{(A, M) /(R, P)}^{*} \rightarrow \mathrm{HH}_{*}^{(R, P)}(A, M)
$$

of graded commutative $A$-algebras. 
Proposition 5.28 When $(A, M)$ is $\log$ smooth over $(R, P)$, so that $\Omega_{(A, M) /(R, P)}^{1}$ is a finitely generated projective $A$-module, then the canonical map (5-1) is an isomorphism.

Remark 5.29 We plan to prove this result, together with its topological generalization for $\log$ THH smooth $(R, P) \rightarrow(A, M)$, in joint work with Philipp Reinhard. The idea is to construct a log Quillen spectral sequence, analogous to Quillen [60, (8.2)], Minasian [51, 2.7] and McCarthy-Minasian [48, 1.1] in the classical cases.

\section{Part II Logarithmic structures on structured ring spectra}

\section{Topological foundations}

We now promote the algebraic theory of the previous part to a topological setting, where rings are replaced by structured ring spectra and monoids are replaced by structured $H$-spaces. In fact, we have at least two choices of topological foundations, based on the work of Peter May et al [21] and of Jeff Smith et al [32], respectively, so we begin by reviewing these. We emphasize the topological analogues of the categories, functors and adjunctions that played key roles in Sections 2 through 5. A third choice of foundations, in the context of infinity-categories, with better formal properties when it comes to adjunctions, has been contemplated by Clark Barwick, but we cannot discuss its details in this review.

Definition 6.1 Let $\mathcal{U}$ be the category of (compactly generated weak Hausdorff) unbased topological spaces and continuous maps. Let $\mathcal{T}$ be the category of (compactly generated weak Hausdorff) based topological spaces and base-point preserving continuous maps. Let $\mathcal{M}_{S}$ be the category of $S$-modules, in the sense of Elmendorf-KrizMandell-May [21, Section II.1]. There are adjunctions

$$
S[-]: \mathcal{U} \stackrel{(-)_{+}}{\longleftrightarrow} \mathcal{T} \underset{\Omega^{\infty}}{\stackrel{\Sigma^{\infty}}{\rightleftarrows}} \mathcal{M}_{S}: \Omega^{\infty}
$$

with the left adjoints on top, where the unlabeled arrow is the forgetful functor. We write $X \mapsto S[X]=\Sigma^{\infty}\left(X_{+}\right)$for the composite functor $\mathcal{U} \rightarrow \mathcal{M}_{S}$, and $E \mapsto \Omega^{\infty} E$ for the composite functor $\mathcal{M}_{S} \rightarrow \mathcal{U}$, so that $S[-]$ is left adjoint to $\Omega^{\infty}$.

The suspension spectrum functor $\Sigma^{\infty}: \mathcal{T} \rightarrow \mathcal{M}_{S}$ is the composite of the suspension prespectrum functor $\Sigma^{\infty}$ from $\mathcal{T}$ to Lewis-May prespectra $\mathcal{P} U$ on a fixed universe $U$ (a countably infinite dimensional inner product space), the spectrification functor $L$ to 
Lewis-May spectra $\mathcal{S} U$, the free functor $\mathbb{L}$ to $\mathbb{L}$-spectra, and the functor $S \wedge_{\mathcal{L}}(-)$ to $S$-modules. By Lewis-May (pre-)spectra, we mean the nonequivariant form of the $G$-(pre-)spectra discussed in Lewis-May-Steinberger [39, Section I.2]. The underlying infinite loop space functor $\Omega^{\infty}: \mathcal{M}_{S} \rightarrow \mathcal{T}$ is the composite of the functor $F_{\mathcal{L}}(S,-)$ to $\mathbb{L}$-spectra, the forgetful functors to spectra and prespectra, and evaluation at the zero-th indexing space 0 in the universe.

Definition 6.2 The cartesian product of spaces, resp. the smash products of based spaces and of $S$-modules, turn $(\mathcal{U}, \times, *),\left(\mathcal{T}, \wedge, S^{0}\right)$ and $\left(\mathcal{M}_{S}, \wedge, S\right)$ into symmetric monoidal categories. Let $\mathcal{C}_{S}$ be the category of commutative $S$-algebras, ie, the commutative monoids in $\mathcal{M}_{S}$. For a fixed commutative $S$-algebra $A$, let $\mathcal{C}_{A}=A / \mathcal{C}_{S}$ be the category of commutative $A$-algebras, ie, the commutative $S$-algebras under $A$.

Let $\mathcal{L}$ be the linear isometries operad (in $(\mathcal{U}, \times, *)$ ) associated to the fixed universe $U$, with $j$-th space $\mathcal{L}(j)$ equal to the contractible space of linear isometries $U^{j} \rightarrow U$. Following May [47, Section 3] we let $\mathcal{L}_{+}$be the operad in $\left(\mathcal{T}, \wedge, S^{0}\right)$ with $j$-th space $\mathcal{L}(j)_{+}$, adding a disjoint zero. The underlying Lewis-May spectrum of each commutative $S$-algebra $A$ has a canonical $\mathcal{L}$-action, with structure map

$$
\bigvee_{j \geq 0} \mathcal{L}(j) \ltimes \Sigma_{j} A^{\wedge j} \rightarrow A,
$$

making it an $E_{\infty}$ ring spectrum for the $E_{\infty}$ operad $\mathcal{L}$; see [21, Section II.4]. These are homotopy commutative ring spectra satisfying coherence conditions of all orders. Evaluating on zero-th spaces, one finds that $\Omega^{\infty} A$ has a canonical $\mathcal{L}_{+}$-action

$$
\bigvee_{j \geq 0} \mathcal{L}(j)_{+} \wedge_{\Sigma_{j}} \Omega^{\infty}(A)^{\wedge j} \rightarrow \Omega^{\infty} A
$$

in $\left(\mathcal{T}, \wedge, S^{0}\right)$ that makes it an $\mathcal{L}_{0}$-space. Applying the forgetful functor to unbased spaces, there is a canonical $\mathcal{L}$-action

$$
\coprod_{j \geq 0} \mathcal{L}(j) \times_{\Sigma_{j}} \Omega^{\infty}(A)^{j} \rightarrow \Omega^{\infty} A
$$

in $(\mathcal{U}, \times, *)$ that makes $\Omega^{\infty} A$ an $\mathcal{L}$-space. To emphasize that we retain the (multiplicative) $\mathcal{L}$ - or $\mathcal{L}_{+}$-action on $\Omega^{\infty} A$, we denote it by $\Omega_{\otimes}^{\infty} A$.

Let $\mathcal{L}_{0}[\mathcal{T}]$ be the category of $\mathcal{L}_{0}$-spaces in $\mathcal{T}$, less formally known as $E_{\infty}$ spaces with zero [46, Section IV.1], and let $\mathcal{L}[\mathcal{U}]$ be the category of $\mathcal{L}$-spaces in $\mathcal{U}$, similarly known as $E_{\infty}$ spaces. These are homotopy commutative $H$-spaces satisfying coherence 
conditions of all orders. There are two composable adjunctions

$$
S[-]: \mathcal{L}[\mathcal{U}] \stackrel{(-)_{+}}{\longleftarrow} \mathcal{L}_{0}[\mathcal{T}] \underset{\Omega_{\otimes}^{\infty}}{\stackrel{\Sigma^{\infty}}{\longrightarrow}} \mathcal{C}_{S}: \Omega_{\otimes}^{\infty}
$$

as before, where all functors are compatible with those in Definition 6.1 via the functors that forget the multiplicative structure. For example, given an $\mathcal{L}$-space $M$, the unreduced suspension spectrum $S[M]$ is the commutative $S$-algebra with $\mathcal{L}$-action

$$
\bigvee_{j \geq 0} \mathcal{L}(j) \ltimes_{\Sigma_{j}}\left(\Sigma^{\infty} M_{+}\right)^{\wedge j} \cong \Sigma^{\infty}\left(\coprod_{j \geq 0} \mathcal{L}(j) \times M^{j}\right)_{+} \rightarrow \Sigma^{\infty} M_{+}
$$

on its underlying Lewis-May spectrum.

Definition 6.3 There are free functors

$$
L: \mathcal{U} \rightarrow \mathcal{L}[\mathcal{U}], \quad L_{0}: \mathcal{T} \rightarrow \mathcal{L}_{0}[\mathcal{T}] \quad \text { and } \quad P: \mathcal{M}_{S} \rightarrow \mathcal{C}_{S}
$$

defined by $L X=\bigsqcup_{j \geq 0} \mathcal{L}(j) \times \Sigma_{j} X^{j}, L_{0} Y=\bigvee_{j \geq 0} \mathcal{L}(j)_{+} \wedge_{\Sigma_{j}} Y^{\wedge j}$ and $P E=$ $\bigvee_{j \geq 0} E^{\wedge j} / \Sigma_{j}$, for $X$ in $\mathcal{U}, Y$ in $\mathcal{T}$ and $E$ in $\mathcal{M}_{S}$. These three functors are left adjoint to the forgetful functors $\mathcal{L}[\mathcal{U}] \rightarrow \mathcal{U}, \mathcal{L}_{0}[\mathcal{T}] \rightarrow \mathcal{T}$ and $\mathcal{C}_{S} \rightarrow \mathcal{M}_{S}$, respectively.

Remark 6.4 There are topological model structures on the categories $\mathcal{U}, \mathcal{T}$ and $\mathcal{M}_{S}$, such that cofibrations are retracts of relative cell objects, weak equivalences have the usual meaning, and fibrations are Serre fibrations [21, Section VII.4]. The two composable adjunctions in Definition 6.1 form Quillen pairs, hence induce weak equivalences between the derived (= homotopically meaningful) mapping spaces, such as

$$
\mathcal{M}_{S}(S[X], E) \simeq \mathcal{T}\left(X_{+}, \Omega^{\infty} E\right) \simeq \mathcal{U}\left(X, \Omega^{\infty} E\right) .
$$

Furthermore, there are topological model structures on the categories $\mathcal{L}[\mathcal{U}], \mathcal{L}_{0}[\mathcal{T}]$ and $\mathcal{C}_{S}$, as explained in [21, Section VII.4], such that the two composable adjunctions in Definition 6.2 consist of Quillen pairs. Hence there are weak equivalences of (derived) mapping spaces

$$
\mathcal{C}_{S}(S[M], A) \simeq \mathcal{L}_{0}[\mathcal{T}]\left(M_{+}, \Omega_{\otimes}^{\infty} A\right) \simeq \mathcal{L}[\mathcal{U}]\left(M, \Omega_{\otimes}^{\infty} A\right) .
$$

Lastly, the three adjunctions in Definition 6.3 are also given by Quillen pairs, inducing weak equivalences $\mathcal{L}[\mathcal{U}](L X, M) \simeq \mathcal{U}(X, M)$ for $X$ in $\mathcal{U}$ and $M$ in $\mathcal{L}[\mathcal{U}]$, $\mathcal{L}_{0}[\mathcal{T}]\left(L_{0} Y, N\right) \simeq \mathcal{T}(Y, N)$ for $Y$ in $\mathcal{T}$ and $N$ in $\mathcal{L}_{0}[\mathcal{T}]$, and $\mathcal{C}_{S}(P E, A) \simeq \mathcal{M}_{S}(E, A)$ for $E$ in $\mathcal{M}_{S}$ and $A$ in $\mathcal{C}_{S}$. 
Lemma 6.5 The category $\mathcal{L}[\mathcal{U}]$ is complete and cocomplete, and the formation of limits commutes with the forgetful functor to $\mathcal{U}$. The colimit of a diagram of $\mathcal{L}$-spaces $i \mapsto M_{i}$ is given by the coequalizer

$$
L\left(\operatorname{colim}_{i} L M_{i}\right) \underset{L \xi}{\stackrel{\mu \circ L \kappa}{\longrightarrow}} L\left(\operatorname{colim}_{i} M_{i}\right)
$$

formed in $\mathcal{U}$, where colim is the colimit in $\mathcal{U}, \kappa: \operatorname{colim}_{i} L M_{i} \rightarrow L\left(\operatorname{colim}_{i} M_{i}\right)$ is the canonical map, $\mu: L L \rightarrow L$ expresses composition in $\mathcal{L}$, and $\xi$ is the colimit of the structure maps $\xi_{i}: L M_{i} \rightarrow M_{i}$

The coproduct of cofibrant $M_{1}$ and $M_{2}$ in $\mathcal{L}[\mathcal{U}]$ is weakly equivalent to the cartesian product $M_{1} \times M_{2}$, via the canonical map $M_{1} \amalg M_{2} \rightarrow M_{1} \times M_{2}$.

Similar statements hold for limits, colimits and coproducts in $\mathcal{L}_{0}[\mathcal{T}]$, relative to $\mathcal{T}$, using the limits, colimits and smash products in $\mathcal{T}$.

Proof Being a right adjoint, the forgetful functor commutes with limits. The existence of colimits in $\mathcal{L}[\mathcal{U}]$, and the expression for the colimit in terms of a (reflexive) coequalizer, follow as in [21, II.7.4]. The monad $L$ preserves reflexive coequalizers by [21, II.7.2]. See Basterra-Mandell [13, 6.8] for the weak equivalence of the coproduct and cartesian product.

Definition 6.6 Let $\mathcal{L}[\mathcal{U}]^{\mathrm{gp}} \subset \mathcal{L}[\mathcal{U}]$ be the full subcategory of grouplike $\mathcal{L}$-spaces. For each $\mathcal{L}$-space $M$ let $F M$ be the grouplike sub- $\mathcal{L}$-space consisting of the homotopy invertible elements in $M$. See May [46, Section III.2]. The inclusion $\iota: F M \rightarrow M$ is the embedding of a set of full path components, and is therefore a fibration. The resulting functor $F: \mathcal{L}[\mathcal{U}] \rightarrow \mathcal{L}[\mathcal{U}]^{\mathrm{gp}}$ is right adjoint to the forgetful functor, with $\iota: F M \rightarrow M$ as the adjunction counit. For each commutative $S$-algebra $A$ we write $\mathrm{GL}_{1}(A)$ for the grouplike $\mathcal{L}$-space $F \Omega_{\otimes}^{\infty} A$. There is a pullback square

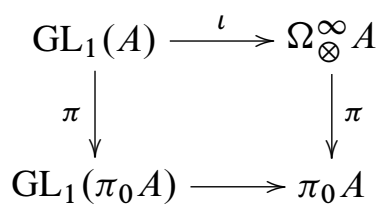

of $\mathcal{L}$-spaces, where the vertical maps take a point to its path component, and the horizontal maps are inclusions.

Definition 6.7 Let $\mathcal{C}_{\infty}$ be the little $\infty$-cubes operad, with $j$-th space $\mathcal{C}_{\infty}(j)$ the colimit over $n$ of the space $\mathcal{C}_{n}(j)$ of $j$ little $n$-cubes in $I^{n}=[0,1]^{n}$. See May 
[45, Section 4]. Like $\mathcal{L}, \mathcal{C}_{\infty}$ is an $E_{\infty}$ operad. Let $\mathcal{C}_{\infty}[\mathcal{U}]$ be the category of $\mathcal{C}_{\infty}-$ spaces, and let $\mathcal{C}_{\infty}[\mathcal{U}]^{\text {gp }}$ be the full subcategory of grouplike $\mathcal{C}_{\infty}$-spaces. To each $\mathcal{C}_{\infty}[\mathcal{U}]$-space $M$ there is an associated prespectrum $B^{\infty} M=\left\{n \mapsto B^{n} M\right\}$, with $n$-th space $B^{n} M$ given by a monadic bar construction $B\left(\Sigma^{n}, C_{n}, M\right)$. See May [45, Section 9, Section 13]. Here $C_{n} X=\bigsqcup_{j \geq 0} \mathcal{C}_{n}(j) \times{ }_{\Sigma_{j}} X^{j} / \simeq_{1}$ denotes the free $\mathcal{C}_{n}$-space on a unit-pointed space $(X, 1)$, so $C_{n} S^{0}=\bigsqcup_{j \geq 0} \mathcal{C}_{n}(j) / \Sigma_{j}$, for example. The adjoint structure maps $B^{n} M \rightarrow \Omega B^{n+1} M$ are weak equivalences for $n \geq 1$. The associated infinite loop space $\Gamma M=\Omega^{\infty} B^{\infty} M$ is grouplike, and this construction defines a group completion functor $\Gamma: \mathcal{C}_{\infty}[\mathcal{U}] \rightarrow \mathcal{C}_{\infty}[\mathcal{U}]^{\mathrm{gp}}$. At the level of homotopy categories, $\operatorname{Ho}(\Gamma): \operatorname{Ho}\left(\mathcal{C}_{\infty}[\mathcal{U}]\right) \rightarrow \operatorname{Ho}\left(\mathcal{C}_{\infty}[\mathcal{U}]^{\mathrm{gp}}\right)$ is left adjoint to the forgetful functor $\operatorname{Ho}\left(\mathcal{C}_{\infty}[\mathcal{U}]^{\mathrm{gp}}\right) \rightarrow \operatorname{Ho}\left(\mathcal{C}_{\infty}[\mathcal{U}]\right)$, but this adjunction does not strictly lift to $\mathcal{C}_{\infty}[\mathcal{U}]$ and $\mathcal{C}_{\infty}[\mathcal{U}]^{\mathrm{gp}}$. Still, there is a natural group completion map $\gamma: M \rightarrow \Gamma M$, which induces the adjunction unit at the level of homotopy categories.

Using the chain $\mathcal{C}_{\infty} \leftarrow \mathcal{C}_{\infty} \times \mathcal{L} \rightarrow \mathcal{L}$ of maps of $E_{\infty}$ operads, it is possible to define two adjunctions

$$
\mathcal{L}[\mathcal{U}] \longleftrightarrow\left(\mathcal{C}_{\infty} \times \mathcal{L}\right)[\mathcal{U}] \longleftrightarrow \mathcal{C}_{\infty}[\mathcal{U}]
$$

that induce a chain of equivalences at the level of homotopy categories, but which do not compose to a direct adjunction between $\mathcal{L}$-spaces and $\mathcal{C}_{\infty}$-spaces. Stringing these constructions together we get a group completion functor $\Gamma: \mathcal{L}[\mathcal{U}] \rightarrow \mathcal{L}[\mathcal{U}]^{\mathrm{gp}}$, with a natural map $\gamma: M \rightarrow \Gamma M$ that is a weak equivalence when $M$ is grouplike. Again, this $\operatorname{Ho}(\Gamma)$ is left adjoint to the forgetful functor $\operatorname{Ho}\left(\mathcal{L}[\mathcal{U}]^{\mathrm{gp}}\right) \rightarrow \operatorname{Ho}(\mathcal{L}[\mathcal{U}])$, but its lift $\Gamma$ is not an adjoint in the strict sense.

Remark 6.8 We may also view $\mathcal{L}$ as a non $-\Sigma$ operad, in which case it is an $A_{\infty}$ operad. The underlying Lewis-May spectrum of an associative $S$-algebra $A$ has a canonical non- $\Sigma \mathcal{L}$-action, so $\Omega_{\otimes}^{\infty} A$ is a non- $\Sigma \mathcal{L}_{0}$-space, ie, an $A_{\infty}$ space with zero. Forgetting the special role of 0 , it is also a non- $\Sigma \mathcal{L}$-space, ie, an $A_{\infty}$ space. The homotopy units $F \Omega_{\otimes}^{\infty} A=\mathrm{GL}_{1}(A)$ form a grouplike non- $\Sigma \mathcal{L}$-space, and we can group complete a non- $\Sigma \mathcal{L}$-space by passing from $\mathcal{L}$ to the non- $\Sigma$ operad of "little ordered intervals", which has the same algebras as the ordinary operad $\mathcal{C}_{1}$. For $\mathcal{C}_{1}$-spaces $M, B M=B\left(\Sigma, C_{1}, M\right)$ and $\Gamma M=\Omega B M$ still make sense.

Definition 6.9 Let $\mathcal{S}$ be the category of simplicial sets, let $\mathcal{S}_{0}$ be the category of based simplicial sets, and let $\mathcal{S} p^{\Sigma}$ be the category of symmetric spectra in the sense of Hovey-Shipley-Smith [32]. We view symmetric spectra as right modules over the sphere spectrum $S$.

We now follow Schlichtkrull [68, Section 2; 69, Section 2]. Let $\mathcal{I}$ be the skeleton category of finite sets and injective functions, with one object $n$ for each integer $n \geq 0$, 
and morphism sets $\mathcal{I}(m, n)$ equal to the set of injective functions $\alpha:\{1, \ldots, m\} \rightarrow$ $\{1, \ldots, n\}$. Let $\mathcal{S}^{\mathcal{I}}$ be the category of $\mathcal{I}$-spaces, ie, functors $X: \mathcal{I} \rightarrow \mathcal{S}$, and let $\mathcal{S}_{0}^{\mathcal{I}}$ be the category of based $\mathcal{I}$-spaces, ie, functors $Y: \mathcal{I} \rightarrow \mathcal{S}_{0}$. The permutations $\Sigma_{n}=$ $\mathcal{I}(n, n)$ act from the left on $Y_{n}=Y(n)$, and the inclusion $\{1, \ldots, n\} \rightarrow\{1, \ldots, n, n+1\}$ induces a stabilization map $Y_{n} \rightarrow Y_{n+1}$. There are two composable adjunctions

$$
S[-]: \mathcal{S}^{\mathcal{I}} \stackrel{(-)_{+}}{\longleftarrow} \mathcal{S}_{0}^{\mathcal{I}} \underset{\Omega^{\bullet}}{\stackrel{\Sigma^{\bullet}}{\longleftrightarrow}} \mathcal{S} p^{\Sigma}: \Omega^{\bullet}
$$

where the unlabeled arrow is the forgetful functor. The functor $(-)_{+}$takes $X$ in $\mathcal{S}^{\mathcal{I}}$ to $X_{+}$with $X_{+}(n)=X(n)_{+}$. The functor $\Sigma^{\bullet}$ takes $Y$ in $\mathcal{S}_{0}^{\mathcal{I}}$ to the symmetric spectrum with $n$-th space $\Sigma^{n} Y_{n}=Y_{n} \wedge S^{n}$, with the diagonal $\Sigma_{n}$-action and structure maps $\sigma: \Sigma\left(Y_{n} \wedge S^{n}\right) \rightarrow Y_{n+1} \wedge S^{n+1}$ induced by the stabilization map above. The functor $\Omega^{\bullet}$ takes $E$ in $\mathcal{S} p^{\Sigma}$ to a based $\mathcal{I}$-space $\Omega^{\bullet} E$, with $n$-th space $\Omega^{n} E_{n}$. Each $\alpha$ in $\mathcal{I}(m, n)$ induces the map $\alpha_{*}: \Omega^{m} E_{m} \rightarrow \Omega^{n} E_{n}$ taking $f: S^{m} \rightarrow E_{m}$ to the composite

$$
S^{n} \stackrel{\gamma^{-1}}{\longrightarrow} S^{m} \wedge S^{n-m} \stackrel{f \wedge 1}{\longrightarrow} E_{m} \wedge S^{n-m} \stackrel{\sigma}{\rightarrow} E_{n} \stackrel{\gamma}{\rightarrow} E_{n},
$$

where $\gamma: n \rightarrow n$ is any choice of permutation that extends $\alpha$.

Remark 6.10 The colimit-over- $\mathcal{I}$ functor $\operatorname{colim}_{\mathcal{I}}: \mathcal{S}^{\mathcal{I}} \rightarrow \mathcal{S}$ is left adjoint to the constant- $\mathcal{I}$-space functor $\mathcal{S} \rightarrow \mathcal{S}^{\mathcal{I}}$. In the model structures of Christian Schlichtkrull and Steffen Sagave [71], [67], this adjoint pair is a Quillen equivalence, so that we can view $\mathcal{I}$-spaces as an equivalent model for simplicial sets (or topological spaces). Similar remarks apply for based $\mathcal{I}$-spaces, based simplicial sets and based spaces.

The reason for working with $\mathcal{I}$-spaces in place of spaces has to do with the monoidal structures, since commutative monoids in $\mathcal{I}$-spaces model arbitrary $E_{\infty}$ spaces, whereas commutative monoids in ordinary spaces become products of EilenbergMac Lane spaces upon group completion.

For noncofibrant $\mathcal{I}$-spaces the correct homotopy type is computed by the colimit over $\mathcal{I}$ of a cofibrant replacement, ie, by the homotopy colimit $X_{h \mathcal{I}}=\operatorname{hocolim}_{\mathcal{I}} X$. In particular, a map $X \rightarrow Y$ of $\mathcal{I}$-spaces is a weak equivalence if and only if $X_{h \mathcal{I}} \rightarrow Y_{h \mathcal{I}}$ is a weak equivalence of simplicial sets (or spaces). In line with this characterization, the homotopy groups of $X$ are defined to be the homotopy groups of $X_{h \mathcal{I}}$. An $\mathcal{I}$-space $X$ is positively fibrant if each simplicial set $X(n)$ is fibrant, and for each morphism $\alpha: m \rightarrow n$ in $\mathcal{I}$ with $m \geq 1$ the map $\alpha_{*}=X(\alpha): X(m) \rightarrow X(n)$ is a weak equivalence. Let $\mathcal{N} \subset \mathcal{I}$ be the subcategory with the same objects, but only the inclusions $\alpha:\{1, \ldots, m\} \rightarrow\{1, \ldots, n\}$ (with $\alpha(i)=i$ for all $i$ ) as morphisms. An $\mathcal{I}$-space $X$ is semistable if the canonical map hocolim $\mathcal{N}_{\mathcal{N}} X \rightarrow \operatorname{hocolim}_{\mathcal{I}} X$ is a 
weak equivalence. Positively fibrant $\mathcal{I}$-spaces are semistable, since the nerve of $\mathcal{I}$ is contractible. See Schwede [73, Section I.4.5] for a discussion of semistability in the context of symmetric spectra, and Schlichtkrull [71] for the case of $\mathcal{I}$-spaces.

Definition 6.11 The concatenation $\sqcup$ of finite sets turns $(\mathcal{I}, \sqcup, 0)$ into a symmetric monoidal category, so the functor categories $\mathcal{S}^{\mathcal{I}}$ and $\mathcal{S}_{0}^{\mathcal{I}}$ inherit symmetric monoidal pairings from the cartesian product in $\mathcal{S}$ and the smash product in $\mathcal{S}_{0}$, respectively. For $X_{1}$ and $X_{2}$ in $\mathcal{S}^{\mathcal{I}}$, we write $X_{1} \otimes X_{2}$ for this product,

$$
\left(X_{1} \otimes X_{2}\right)(n)=\operatorname{colim}_{n_{1} \sqcup n_{2} \rightarrow n} X_{1}\left(n_{1}\right) \times X_{2}\left(n_{2}\right),
$$

defined as the left Kan extension of the composite

$$
\mathcal{I} \times \mathcal{I} \stackrel{X_{1} \times X_{2}}{\longrightarrow} \mathcal{S} \times \mathcal{S} \stackrel{\times}{\rightarrow} \mathcal{S}
$$

along $\sqcup: \mathcal{I} \times \mathcal{I} \rightarrow \mathcal{I}$. In lack of a better symbol, we write $Y_{1} \square Y_{2}$ for the smash product in $\mathcal{S}_{0}^{\mathcal{I}}$ of two based $\mathcal{I}$-spaces $Y_{1}$ and $Y_{2}$. We keep the standard notation $E_{1} \wedge E_{2}$ for the smash product of two symmetric spectra.

Let $\mathcal{C S}^{\mathcal{I}}$ be the category of commutative monoids in $\mathcal{I}$-spaces and let $\mathcal{C} \mathcal{S}_{0}^{\mathcal{I}}$ be the category of commutative monoids in based $\mathcal{I}$-spaces. We usually refer to these as commutative $\mathcal{I}$-space monoids and commutative based $\mathcal{I}$-space monoids, respectively. Let $\mathcal{C S} p^{\Sigma}$ be the category of commutative monoids in symmetric spectra, ie, the commutative symmetric ring spectra. For a fixed commutative symmetric ring spectrum $R$, let $\mathcal{C}_{R}=R / \mathcal{C S} p^{\Sigma}$ be the category of commutative $R$-algebras, ie, the commutative symmetric ring spectra under $R$.

The left adjoints $(-)_{+}$and $\Sigma^{\bullet}$ in Definition 6.9 are strong monoidal, and the right adjoints (the forgetful functor and $\Omega^{\bullet}$ ) are (lax) monoidal; see Mac Lane [44, Section XI.2]. Hence there are composable adjunctions

$$
S[-]: \mathcal{C S}^{\mathcal{I}} \stackrel{(-)_{+}}{\longleftrightarrow} \mathcal{C} \mathcal{S}_{0}^{\mathcal{I}} \underset{\Omega_{\otimes}^{\bullet}}{\stackrel{\Sigma^{\bullet}}{\longrightarrow}} \mathcal{C S} p^{\Sigma}: \Omega_{\otimes}^{\bullet}
$$

relating the three categories of commutative monoids. Note that we write $\Omega_{\otimes}^{\bullet} A$ for $\Omega^{\bullet} A$ equipped with the commutative monoidal structure inherited from that on $A$.

Remark 6.12 For a commutative monoid $M$ in $\mathcal{I}$-spaces, $M_{h \mathcal{I}}=\operatorname{hocolim}_{\mathcal{I}} M$ has a canonical action by the Barratt-Eccles operad $E \Sigma$, with $j$-th space $E \Sigma_{j}[70$, 6.5]. This is an $E_{\infty}$ operad, and the functor hocolim ${ }_{I}: \mathcal{C S}^{\mathcal{I}} \rightarrow E \Sigma[\mathcal{U}]$ induces an equivalence of homotopy- and infinity-categories. Hence we can view commutative 
monoids in $\mathcal{I}$-spaces as a model for $E_{\infty}$ spaces. Similarly, commutative monoids in based $\mathcal{I}$-spaces are a model for $E_{\infty}$ spaces with zero.

Definition 6.13 There are free functors

$$
C: \mathcal{S}^{\mathcal{I}} \rightarrow \mathcal{C} \mathcal{S}^{\mathcal{I}}, \quad C_{0}: \mathcal{S}_{0}^{\mathcal{I}} \rightarrow \mathcal{C} \mathcal{S}_{0}^{\mathcal{I}} \quad \text { and } \quad P: \mathcal{S} p^{\Sigma} \rightarrow \mathcal{C S} p^{\Sigma},
$$

defined by $C X=\bigsqcup_{j \geq 0} X^{\bigotimes j} / \Sigma_{j}, C_{0} Y=\bigvee_{j \geq 0} Y^{\square j} / \Sigma_{j}$ and $P E=\bigvee_{j \geq 0} E^{\wedge j} / \Sigma_{j}$. These are left adjoint to the respective forgetful functors. In the definition of $C X$, $X^{\otimes j}$ denotes the $j$-fold product $X \otimes \cdots \otimes X$ formed in $\mathcal{S}^{\mathcal{I}}$, and similarly for $Y^{\boxminus j}=Y \boxminus \cdots \boxminus Y$ in $\mathcal{S}_{0}^{\mathcal{I}}$.

Remark 6.14 There are simplicial model structures on the categories $\mathcal{S}^{\mathcal{I}}, \mathcal{S}_{0}^{\mathcal{I}}$ and $\mathcal{S} p^{\Sigma}$ by Sagave-Schlichtkrull [67] and Schwede [73, Section III.2], such that the adjunctions in Definition 6.9 form Quillen pairs, with induced weak equivalences

$$
\mathcal{S} p^{\Sigma}(S[X], E) \simeq \mathcal{S}_{0}^{\mathcal{I}}\left(X_{+}, \Omega^{\bullet} E\right) \simeq \mathcal{S}^{\mathcal{I}}\left(X, \Omega^{\bullet} E\right)
$$

(after cofibrant and fibrant replacements of $X$ and $E$, respectively). Here we have in mind the positive (projective, stable) model structures, where a symmetric spectrum $E$ is positively fibrant if each simplicial set $E_{n}$ is fibrant for $n \geq 0$, and each adjoint structure map $E_{n} \rightarrow \Omega E_{n+1}$ is a weak equivalence for $n \geq 1$.

There are corresponding (projective) model structures on $\mathcal{C} \mathcal{S}^{\mathcal{I}}, \mathcal{C} \mathcal{S}_{0}^{\mathcal{I}}$ and $\mathcal{C} \mathcal{S} p^{\Sigma}$, such that the adjunctions in Definitions 6.11 and 6.13 form Quillen pairs. Hence there are weak equivalences of derived mapping spaces

$$
\mathcal{C S} p^{\Sigma}(S[M], A) \simeq \mathcal{C} \mathcal{S}_{0}^{\mathcal{I}}\left(M_{+}, \Omega_{\otimes}^{\bullet} A\right) \simeq \mathcal{C S}^{\mathcal{I}}\left(M, \Omega_{\otimes}^{\bullet} A\right)
$$

and $\mathcal{C S}^{\mathcal{I}}(C X, M) \simeq \mathcal{S}^{\mathcal{I}}(X, M), \mathcal{C S}_{0}^{\mathcal{I}}\left(C_{0} Y, N\right) \simeq \mathcal{S}_{0}^{\mathcal{I}}(Y, N)$ and $\mathcal{C S} p^{\Sigma}(P E, A) \simeq$ $\mathcal{S} p^{\Sigma}(E, A)$.

Lemma 6.15 The category $\mathcal{C} \mathcal{S}^{\mathcal{I}}$ is complete and cocomplete, and the formation of limits commutes with the forgetful functor to $\mathcal{S}^{\mathcal{I}}$. The coproduct $M_{1} \otimes M_{2}$ of $M_{1}$ and $M_{2}$ in $\mathcal{C S}^{\mathcal{I}}$ is weakly equivalent to the cartesian product $M_{1} \times M_{2}$, for cofibrant and semistable $M_{1}$ and $M_{2}$. Similar statements hold for $\mathcal{C S}_{0}^{\mathcal{I}}$, where the coproduct $N_{1} \unrhd N_{2}$ of $N_{1}$ and $N_{2}$ is weakly equivalent to the smash product $N_{1} \wedge N_{2}$, for cofibrant and semistable $N_{1}$ and $N_{2}$.

Proof The unit maps $* \rightarrow M_{1}$ and $* \rightarrow M_{2}$ induce the structure maps $M_{1}=$ $M_{1} \otimes * \rightarrow M_{1} \otimes M_{2}$ and $M_{2}=* \otimes M_{2} \rightarrow M_{1} \otimes M_{2}$ that express $M_{1} \otimes M_{2}$ as 
the coproduct in $\mathcal{C S}^{\mathcal{I}}$ of $M_{1}$ and $M_{2}$. The pairing $M_{1} \otimes M_{1} \rightarrow M_{1}$ specifies maps $M_{1}\left(n_{1}\right) \times M_{1}\left(n_{2}\right) \rightarrow M_{1}\left(n_{1} \sqcup n_{2}\right)$, and similarly for $M_{2}$, so there are maps

$$
\begin{aligned}
\left(M_{1} \times M_{2}\right)\left(n_{1}\right) \times\left(M_{1} \times M_{2}\right)\left(n_{2}\right) \cong\left(M_{1}\left(n_{1}\right) \times M_{1}\left(n_{2}\right)\right) \times\left(M_{2}\left(n_{1}\right) \times M_{2}\left(n_{2}\right)\right) \\
\rightarrow M_{1}\left(n_{1} \sqcup n_{2}\right) \times M_{2}\left(n_{1} \sqcup n_{2}\right) \cong\left(M_{1} \times M_{2}\right)\left(n_{1} \sqcup n_{2}\right)
\end{aligned}
$$

that make $M_{1} \times M_{2}$ a commutative $\mathcal{I}$-space monoid. There is a natural equivalence

$$
\left(M_{1}\right)_{h \mathcal{I}} \times\left(M_{2}\right)_{h \mathcal{I}} \rightarrow\left(M_{1} \otimes M_{2}\right)_{h \mathcal{I}}
$$

for cofibrant $M_{1}$ and $M_{2}$, and a natural map

$$
\left(M_{1} \times M_{2}\right)_{h \mathcal{I}} \rightarrow\left(M_{1}\right)_{h \mathcal{I}} \times\left(M_{2}\right)_{h \mathcal{I}},
$$

which is a weak equivalence when $M_{1}$ and $M_{2}$ are semistable. See Schlichtkrull [71] for more details.

Definition 6.16 Let $\left(\mathcal{C S}^{\mathcal{I}}\right)^{\text {gp }}$ be the full subcategory of grouplike commutative monoids in $\mathcal{I}$-spaces. These are the commutative monoids in $\mathcal{I}$-spaces $M$ such that the commutative monoid $\pi_{0} M_{h \mathcal{I}}$ is an abelian group. The forgetful functor $\left(\mathcal{C S}^{\mathcal{I}}\right)^{\mathrm{gp}} \rightarrow$ $\mathcal{C S}^{\mathcal{I}}$ admits a left adjoint $F: \mathcal{C S}^{\mathcal{I}} \rightarrow\left(\mathcal{C S}^{\mathcal{I}}\right)^{\text {gp }}$, defined in Schlichtkrull [68]. It takes a commutative monoid $M$ to the grouplike commutative submonoid $F M$, with $F M(n) \subset M(n)$ consisting of the simplices in $M(n)$ that have invertible image in the multiplicative commutative monoid $\pi_{0} M_{h \mathcal{I}}$. We write $\iota: F M \rightarrow M$ for the adjunction counit. It is a fibration, since each inclusion $F M(n) \subset M(n)$ is the embedding of a set of full path components. For each commutative symmetric ring spectrum $A$ we write $\mathrm{GL}_{1}(A)$ for the grouplike commutative monoid in $\mathcal{I}$-spaces $F \Omega_{\otimes}^{\bullet} A$. For positively fibrant (or semistable) $A$ there is a pullback square

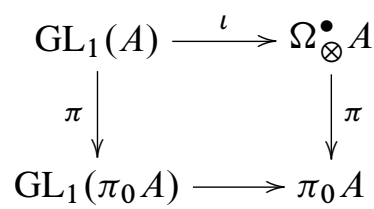

of commutative monoids in $\mathcal{I}$-spaces, since $\pi_{0}\left(\Omega_{\otimes}^{\bullet} A\right)_{h \mathcal{I}} \cong \pi_{0} A$.

Definition 6.17 We can use the (iterated) bar construction to deloop and group complete commutative monoids in $\mathcal{I}$-spaces. For a not necessarily commutative monoid $(M, \mu, \eta)$ in $\left(\mathcal{S}^{\mathcal{I}}, \bigotimes, *\right)$, let the bar construction $B M=B_{\square} M$ be the based $\mathcal{I}$-space obtained by diagonalization from the simplicial $\mathcal{I}$-space

$$
[q] \mapsto M \otimes \cdots \otimes M
$$


( $q$ copies of $M$ ), with face maps induced by $\mu$ and the unique map $M \rightarrow *$, and with degeneracy maps induced by $\eta$, in the usual way. The levelwise suspension $\Sigma M$ of $M$, with $(\Sigma M)(n)=\Sigma(M(n))$, includes into $B M$ as the simplicial 1-skeleton, and there is an adjoint map

$$
\gamma: M \rightarrow \Omega B M
$$

where $\Omega B M$ is the levelwise loop space, with $(\Omega B M)(n)=\Omega(B M(n))$. For positively fibrant (or semistable) $M$ we get that $(\Omega B M)_{h \mathcal{I}} \simeq \Omega B\left(M_{h \mathcal{I}}\right)$, so $\gamma: M \rightarrow$ $\Omega B M$ is a weak equivalence if and only if $M$ is grouplike.

In this generality is not obvious how to use loop sum to make $\Omega B M$ a strictly associative and/or commutative monoid in $\mathcal{I}$-spaces. However, for a commutative $\mathcal{I}$-space monoid $M$ the multiplication $\mu: M \otimes M \rightarrow M$ is an $\mathcal{I}$-space monoid map, so $B M$ is itself a commutative $\mathcal{I}$-space monoid, with multiplication $B \mu: B M \otimes B M \cong$ $B(M \otimes M) \rightarrow B M$. This pairing corresponds to natural maps

$$
B \mu\left(n_{1}, n_{2}\right): B M\left(n_{1}\right) \times B M\left(n_{2}\right) \rightarrow B M\left(n_{1} \sqcup n_{2}\right) .
$$

The pointwise product of loops in $B M$ now makes $\Omega B M$ a commutative $\mathcal{I}$-monoid, with multiplication $\Omega B M \otimes \Omega B M \rightarrow \Omega B M$ given by the maps

$$
\Omega B \mu\left(n_{1}, n_{2}\right): \Omega B M\left(n_{1}\right) \times \Omega B M\left(n_{2}\right) \rightarrow \Omega B M\left(n_{1} \sqcup n_{2}\right)
$$

obtained by looping the maps above. See also Lima-Filho [40, page 134]. Then $\Omega B M$ is a commutative $\mathcal{I}$-space monoid, and $\gamma: M \rightarrow \Omega B M$ is a commutative $\mathcal{I}$-space monoid homomorphism. We give

$$
\Gamma M=\Omega B M
$$

this commutative $\mathcal{I}$-space monoid structure. This defines the group completion functor $\Gamma: \mathcal{C S}^{\mathcal{I}} \rightarrow\left(\mathcal{C S}^{\mathcal{I}}\right)^{\text {gp }}$, which at the level of homotopy categories is left adjoint to the forgetful functor. The group completion map $\gamma: M \rightarrow \Gamma M$ induces the adjunction unit at the level of homotopy categories. See Schlichtkrull [71] for more on group completion of commutative $\mathcal{I}$-space monoids.

For commutative $\mathcal{I}$-space monoids $M$ the bar construction can be iterated arbitrarily often. Letting $B^{0} M=M$ and $B^{n} M=B\left(B^{n-1} M\right)$ for $n \geq 1$ we get a symmetric spectrum

$$
B^{\bullet} M=\left\{n \mapsto B^{n} M\right\}
$$

in the category of based $\mathcal{I}$-spaces, where $\Sigma_{n}$ acts on $B^{n} M$ by permuting the order of the $n$ bar constructions. Applying the homotopy colimit over $\mathcal{I}$, we get a symmetric spectrum

$$
B^{\infty} M=\left(B^{\bullet} M\right)_{h \mathcal{I}}=\left\{n \mapsto\left(B^{n} M\right)_{h \mathcal{I}}\right\}
$$


in (based) simplicial sets. For the positively fibrant (or semistable) $M$ mentioned above, the adjoint structure maps $B^{n} M \rightarrow \Omega B^{n+1} M$ are weak equivalences for $n \geq 1$, so we get a weak equivalence

$$
(\Gamma M)_{h \mathcal{I}} \stackrel{\simeq}{\longrightarrow} \underset{n}{\operatorname{hocolim}} \Omega^{n}\left(B^{n} M\right)_{h \mathcal{I}} .
$$

We think of $B^{\infty} M=\left(B^{\bullet} M\right)_{h \mathcal{I}}$ as the prespectrum associated to the commutative $\mathcal{I}$-space monoid $M$, with underlying infinite loop space weakly equivalent to the group completion $(\Gamma M)_{h \mathcal{I}}$ of $M_{h \mathcal{I}}$.

Remark 6.18 For $M$ as above and $N$ a grouplike commutative $\mathcal{I}$-space monoid, there is a chain of weak equivalences

$$
\mathcal{C S}^{\mathcal{I}}(M, N) \simeq\left(\mathcal{C S}^{\mathcal{I}}\right)^{\mathrm{gp}}(\Gamma M, \Gamma N) \simeq\left(\mathcal{C S}^{\mathcal{I}}\right)^{\mathrm{gp}}(\Gamma M, N)
$$

since $\gamma: N \rightarrow \Gamma N$ is a weak equivalence, so $\Gamma$ is left adjoint to the forgetful functor in the infinity-categorical sense.

Definition 6.19 We can also apply a bar construction to certain monoids in based $\mathcal{I}$-spaces, but these do not produce deloopings in the usual sense. The unit for the symmetric monoidal pairing $\square$ in based $\mathcal{I}$-spaces is the constant $\mathcal{I}$-space $S^{0}=\{0,1\}$. For each object $(Y, \epsilon)$ in the category $\mathcal{S}_{0}^{\mathcal{I}} / S^{0}$ of based $\mathcal{I}$-spaces over $S^{0}$ we let $Y_{0}=\epsilon^{-1}(0)$ and $Y_{1}=\epsilon^{-1}(1)$. A not necessarily commutative monoid $(N, \eta, \mu, \epsilon)$ in this category consists of maps $\eta: S^{0} \rightarrow N, \mu: N \triangleright N \rightarrow N$ and $\epsilon: N \rightarrow S^{0}$, subject to unitality and associativity conditions over $S^{0}$. For such $N$ we let the based bar construction $B_{\wedge} N=B_{\square} N$ be the $\mathcal{I}$-space under and over $S^{0}$ obtained by diagonalization from the simplicial based $\mathcal{I}$-space

$$
[q] \mapsto N \boxminus \cdots \boxminus N=N^{\varpi q},
$$

with face maps induced by $\mu$ and $\epsilon$ and degeneracy maps induced by $\eta$, in the usual way. The inclusion of zero-simplices defines a map $S^{0} \rightarrow B_{\wedge} N$, and the product $\epsilon^{\square q}: N^{\square q} \rightarrow\left(S^{0}\right)^{\square q} \cong S^{0}$ defines the retraction $B_{\wedge} N \rightarrow S^{0}$. Note that $\left(B_{\wedge} N\right)_{1}=B\left(N_{1}\right)$ is the usual bar construction, whereas $\left(B_{\wedge} N\right)_{0}$ depends both on $N_{0}$ and $N_{1}$. The simplicial 1-skeleton of $B_{\wedge} N$ is the disjoint union

$$
\Sigma_{S^{0}}(N)=\Sigma\left(N_{0}\right) \sqcup \Sigma\left(N_{1}\right) .
$$

The right adjoint to $\Sigma_{S^{0}}$ is $\Omega_{S^{0}}$, with $\Omega_{S^{0}}(Y)=\Omega\left(Y_{0}\right) \sqcup \Omega\left(Y_{1}\right)$. The inclusion of the simplicial 1-skeleton $\Sigma_{S^{0}} N \rightarrow B_{\wedge} N$ is left adjoint to a map

$$
\gamma: N \rightarrow \Gamma_{\wedge} N=\Omega_{S^{0}} B_{\wedge} N
$$


which is the disjoint union of a map $\gamma_{0}: N_{0} \rightarrow \Omega\left(B_{\wedge} N\right)_{0}$ and the usual group completion map $\gamma_{1}: N_{1} \rightarrow \Omega\left(B_{\wedge} N\right)_{1}=\Omega B\left(N_{1}\right)$.

Now suppose $N$ is a commutative based $\mathcal{I}$-space monoid over $S^{0}$. Then the multiplication $\mu$ : $N \oslash N \rightarrow N$ is a based $\mathcal{I}$-space monoid map over $S^{0}$, so $B_{\wedge} N$ is also a commutative based $\mathcal{I}$-space monoid over $S^{0}$, with multiplication $\mu:\left(B_{\wedge} N\right) \square\left(B_{\wedge} N\right) \cong$ $B_{\wedge}(N \boxminus N) \rightarrow B_{\wedge} N$. The pointwise product of loops in $B_{\wedge} N$ makes $\Omega_{S^{0}} B_{\wedge} N$ a commutative based $\mathcal{I}$-space monoid over $S^{0}$, and $\gamma$ is a morphism in that category.

In the commutative case, the based bar construction can be iterated infinitely often. Letting $B_{\wedge}^{0} N=N$ and $B_{\wedge}^{n} N=B_{\wedge}\left(B_{\wedge}^{n-1} N\right)$ for $n \geq 1$ we get a symmetric spectrum

$$
B_{\wedge}^{\bullet} N=\left\{n \mapsto B_{\wedge}^{n} N\right\}
$$

in the category of $\mathcal{I}$-spaces under and over $S^{0}$, with suspension operator $\Sigma_{S^{0}}$. We can view this as a pair of symmetric spectra in based $\mathcal{I}$-spaces, with $n$-th terms $\left(B_{\wedge}^{n} N\right)_{0}$ and $\left(B_{\wedge}^{n} N\right)_{1}=B^{n}\left(N_{1}\right)$, respectively. Doing a base change along $S^{0} \rightarrow *$ we get a symmetric spectrum

$$
\bar{B}_{\wedge}^{\bullet} N=\left\{n \mapsto \bar{B}_{\wedge}^{n} N=\left(B_{\wedge}^{n} N\right) / S^{0}\right\}
$$

in based $\mathcal{I}$-spaces. Here $\left(B_{\wedge}^{n} N\right) / S^{0} \cong\left(B_{\wedge}^{n} N\right)_{0} \vee\left(B_{\wedge}^{n} N\right)_{1}$. Applying the homotopy colimit over $\mathcal{I}$ we get the ordinary symmetric spectra $\left(B_{\wedge}^{\infty} N\right)_{0}=\left\{n \mapsto\left(B_{\wedge}^{n} N\right)_{0, h \mathcal{I}}\right\}$, $\left(B_{\wedge}^{\infty} N\right)_{1}=\left\{n \mapsto\left(B_{\wedge}^{n} N\right)_{1, h \mathcal{I}}\right\}=B^{\infty}\left(N_{1}\right)$, and

$$
\bar{B}_{\wedge}^{\infty} N=\left\{n \mapsto\left(\bar{B}_{\wedge}^{n} N\right)_{h \mathcal{I}}\right\}
$$

in (based) simplicial sets.

When $N=M_{+}$is obtained from a commutative $\mathcal{I}$-space monoid by adding a disjoint zero, with $\epsilon: N \rightarrow S^{0}$ defined so that $N_{0}=\{0\}$ and $N_{1}=M$, then $N \square N \cong$ $(M \otimes M)_{+}, B_{\wedge}^{n} N \cong\left(B^{n} M\right)_{+}$for all $n \geq 0, \Gamma_{\wedge} N \cong(\Gamma M)_{+}$, and $\bar{B}_{\wedge}^{\infty} N \cong B^{\infty} M$. In particular, $\gamma: N \rightarrow \Gamma_{\wedge} N$ is an equivalence if and only if $M$ is grouplike.

Remark 6.20 An obvious problem is to determine for which $N$ (with nonisolated zero) the map $\gamma: N \rightarrow \Gamma_{\wedge} N=\Omega_{S^{0}} B_{\wedge} N$ is an equivalence. The submonoid $N_{1}$ must be grouplike, since $\left(\Gamma_{\wedge} N\right)_{1}=\Omega B\left(N_{1}\right)$, but the analogous condition on $N_{0}$ with its $N_{1}$-action does not seem to be known.

Definition 6.21 We say that a based $\mathcal{I}$-space $Y$ is conically based if it can be expressed as a pushout $Y=\operatorname{cone}(L) \cup_{L} Y^{\prime}$ in $\mathcal{I}$-spaces, where cone $(L)$ is the unreduced cone on an $\mathcal{I}$-space $L$, so that the cone point of cone $(L)$ corresponds to the base point of $Y$. We call $L$ the link of the base point. The unreduced cone of an $\mathcal{I}$-space is defined pointwise: $\operatorname{cone}(L)(n)=\operatorname{cone}(L(n))=L(n)_{+} \wedge \Delta^{1}$. We 
think of $Y^{\prime}$ as the complement of the base point in $Y$, obtained by puncturing $Y$ at $*$. The property of being conically based is obviously not preserved by most homotopy equivalences. If $Y=X_{+}$has an isolated base point, it is conically based with $L=\varnothing$ the empty $\mathcal{I}$-space and $Y^{\prime}=X$.

If $Y_{1}$ and $Y_{2}$ are conically based, with links $L_{1}$ and $L_{2}$, then $Y_{1} \boxminus Y_{2}$ is also conically based, with link

$$
\left(Y_{1}^{\prime} \otimes L_{2}\right) \cup_{L_{1} \otimes L_{2}}\left(L_{1} \otimes Y_{2}^{\prime}\right)
$$

and $\left(Y_{1} \bowtie Y_{2}\right)^{\prime} \cong Y_{1}^{\prime} \otimes Y_{2}^{\prime}$. By induction, $C_{0} Y$ is conically based if $Y$ is, and $\left(C_{0} Y\right)^{\prime} \cong C\left(Y^{\prime}\right)$.

We say that a based commutative $\mathcal{I}$-space monoid $N=\operatorname{cone}(L) \cup_{L} N^{\prime}$ is conically based if the multiplication $\mu: N \boxminus N \rightarrow N$ takes $N^{\prime} \otimes N^{\prime} \subset N \boxminus N$ to $N^{\prime} \subset N$. In this case $N^{\prime}$ is a commutative $\mathcal{I}$-space monoid. If $N$ is a commutative conically based $\mathcal{I}$-space monoid over $S^{0}$, then so is $B_{\wedge}^{n} N$ for all $n \geq 0$, and $\left(B_{\wedge}^{n} N\right)^{\prime} \cong B^{n}\left(N^{\prime}\right)$.

Lemma 6.22 Let $M=C X$ be free on an $\mathcal{I}$-space $X$, and let $N=C_{0} Y$ be free on a based $\mathcal{I}$-space $Y$. We view $N=C_{0} Y$ as augmented over $S^{0}=C_{0}(*)$ by the map induced by $Y \rightarrow *$, so $N_{1}=\{1\}$. Then there are weak equivalences $B^{\infty} M \simeq S[X]$ and $\bar{B}_{\wedge}^{\infty} N \simeq \Sigma^{\infty} Y$.

If $Y=\operatorname{cone}(L) \cup_{L} Y^{\prime}$ is conically based, then $N=C_{0} Y$ is conically based with $N^{\prime}=C\left(Y^{\prime}\right)$, so $B^{\infty}\left(N^{\prime}\right) \simeq S\left[Y^{\prime}\right]$.

Proof In the based case $B_{\wedge} N=B_{\wedge} C_{0} Y \cong C_{0} \Sigma Y$, so $B_{\wedge}^{n} N \cong C_{0} \Sigma^{n} Y$. The inclusion $\left(\Sigma^{n} Y\right)_{+} \rightarrow C_{0} \Sigma^{n} Y$ is $(2 n-1)$-connected (for cofibrant $Y$ ), so $\Sigma^{\bullet} Y \rightarrow$ $\bar{B}_{\wedge}^{\bullet} N$ is a (stable) equivalence. Passing to homotopy colimits over $\mathcal{I}$ we get the claimed equivalence $\Sigma^{\infty} Y \simeq \bar{B}_{\wedge}^{\infty} N$.

The unbased case follows from the based case by setting $Y=X_{+}$, so that $N=M_{+}$, and noting that $\bar{B}_{\wedge}^{\infty} N=B^{\infty} M$ and $\Sigma^{\bullet} Y=S[X]$.

The conically based case then follows from the unbased case by setting $X=Y^{\prime}$, so that $M=N^{\prime}$.

\section{$7 \quad$ Logarithmic structures in topology}

We now discuss topological analogues of log rings, where the commutative rings are replaced by structured ring spectra (meaning commutative $S$-algebras or commutative symmetric ring spectra) and the commutative monoids are replaced by $E_{\infty}$ spaces (meaning $\mathcal{L}$-spaces or commutative $\mathcal{I}$-space monoids), or $E_{\infty}$ spaces with zero (meaning $\mathcal{L}_{0}$-spaces or commutative based $\mathcal{I}$-space monoids). 
Definition 7.1 Let $A$ be a commutative symmetric ring spectrum. A pre-log structure on $A$ is a pair $(M, \alpha)$ consisting of a commutative $\mathcal{I}$-space monoid $M$ and a map

$$
\alpha: M \rightarrow \Omega_{\otimes}^{\bullet} A
$$

of commutative $\mathcal{I}$-space monoids. Specifying $\alpha$ is equivalent to specifying the left adjoint map

$$
\bar{\alpha}: S[M] \rightarrow A
$$

of commutative symmetric ring spectra. A pre-log symmetric ring spectrum $(A, M, \alpha)$, often abbreviated to $(A, M)$, is a commutative symmetric ring spectrum $A$ with a pre-log structure $(M, \alpha)$. A map

$$
\left(f, f^{b}\right):(A, M, \alpha) \rightarrow(B, N, \beta)
$$

of pre-log symmetric ring spectra consists of a map $f: A \rightarrow B$ of commutative symmetric ring spectra and a map $f^{b}: M \rightarrow N$ of commutative $\mathcal{I}$-space monoids, such that the square

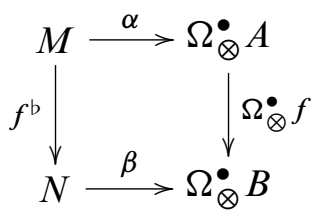

of commutative $\mathcal{I}$-space monoids commutes. In adjoint terms, the condition is that the square

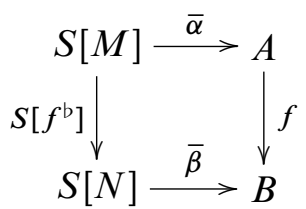

of commutative symmetric ring spectra commutes. A map $\left(f, f^{\text {b }}\right)$ of pre-log symmetric ring spectra is a weak equivalence if $f$ and $f^{b}$ are both weak equivalences.

Let $\operatorname{Pre} \log (S)$ be the resulting category of pre-log symmetric ring spectra. It is equal to the comma-category (or under-category) $\mathcal{C} \mathcal{S}^{\mathcal{I}} / \Omega_{\otimes}^{\bullet}$ associated to

$$
\Omega_{\otimes}^{\bullet}: \mathcal{C S} p^{\Sigma} \rightarrow \mathcal{C S}^{\mathcal{I}}
$$

and isomorphic to the comma-category (or over-category) $S[-] / \mathcal{C S} p^{\Sigma}$ associated to

$$
S[-]: \mathcal{C S}^{\mathcal{I}} \rightarrow \mathcal{C S} p^{\Sigma} .
$$

See Mac Lane [44, Section II.6]. There are forgetful functors from $\operatorname{Pr} \log (S)$ to $\mathcal{C S} p^{\Sigma}$ and $\mathcal{C S}^{\mathcal{I}}$, taking $(A, M)$ to $A$ and $M$, respectively. For a fixed pre-log 
symmetric ring spectrum $(A, M)$, let $\operatorname{Pre} \log (A, M)=(A, M) / \operatorname{Pre} \log (S)$ be the category of pre-log $(A, M)$-algebras, ie, pre-log symmetric ring spectra under $(A, M)$.

Definition 7.2 Let $A$ be a commutative symmetric ring spectrum. A based pre-log structure on $A$ is a pair $(N, \alpha)$ consisting of a commutative based $\mathcal{I}$-space monoid $N$ and a map $\alpha: N \rightarrow \Omega_{\otimes}^{\bullet} A$ of commutative based $\mathcal{I}$-space monoids. Equivalently, a pre-log structure specifies a map $\bar{\alpha}: \Sigma^{\bullet} N \rightarrow A$ of commutative symmetric ring spectra. The category $\operatorname{Pre} \mathcal{L}_{o_{0}}(S)$ of based pre-log symmetric ring spectra is the commacategory $\mathcal{C} \mathcal{S}_{0}^{\mathcal{I}} / \Omega_{\otimes}^{\bullet}$, which is isomorphic to the comma-category $\Sigma^{\bullet} / \mathcal{C S} p^{\Sigma}$. There are obvious forgetful functors from $\operatorname{Pre} \mathcal{L o g}_{0}(S)$ to $\mathcal{C} p^{\Sigma}, \mathcal{C S}_{0}^{\mathcal{I}}$ and $\operatorname{Pre} \log (S)$.

Remark 7.3 Working in commutative $S$-algebras, one may define the category $\operatorname{Pre} \mathcal{L} o g(S)$ of pre-log $S$-algebras as $\mathcal{L}[\mathcal{U}] / \Omega_{\otimes}^{\infty}$, where $\Omega_{\otimes}^{\infty}: \mathcal{C}_{S} \rightarrow \mathcal{L}[\mathcal{U}]$. It is isomorphic to $S[-] / \mathcal{C}_{S}$, where $S[-]: \mathcal{L}[\mathcal{U}] \rightarrow \mathcal{C}_{S}$. In the based setting, the category $\operatorname{Pre} \mathcal{L}_{o_{0}}(S)$ of based pre-log $S$-algebras is defined to be $\mathcal{L}_{0}[\mathcal{T}] / \Omega_{\otimes}^{\infty}$, where $\Omega_{\otimes}^{\infty}: \mathcal{C}_{S} \rightarrow \mathcal{L}_{0}[\mathcal{T}]$. It is isomorphic to $\Sigma^{\infty} / \mathcal{C}_{S}$, where $\Sigma^{\infty}: \mathcal{L}_{0}[\mathcal{T}] \rightarrow \mathcal{C}_{S}$. See also Definition 9.1 below.

For definiteness, we shall mostly work with commutative symmetric ring spectra and commutative $\mathcal{I}$-space monoids, since the description of the coproducts and deloopings of the latter (Definitions 6.11 and 6.17) is notationally a little more convenient than for $\mathcal{L}$-spaces (Lemma 6.5 and Definition 6.7). On the other hand, for more general work with $E_{n}$ ring spectra and $E_{n}$ spaces for $1<n<\infty$, as in Section 9, the operadic point of view is more convenient. Since we are principally interested in multiplicative $E_{\infty}$ spaces (with or without zero), rather than in $E_{\infty}$ ring spaces [46, Section VI.1], we are not directly affected by the consistency issues raised in May [47], although some care in the comparison of definitions is certainly required.

Definition 7.4 Let $\alpha^{-1} \mathrm{GL}_{1}(A) \subset M$ be defined by the pullback square

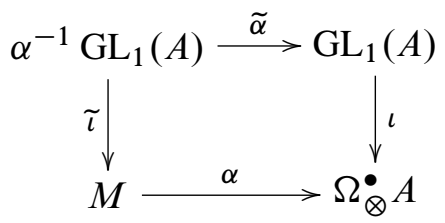

of commutative $\mathcal{I}$-space monoids. The pullback is weakly equivalent to the homotopy pullback, since $\iota$ is a fibration. The pre-log structure $(M, \alpha)$ on $A$ is said to be a $\log$ structure if the restricted map $\tilde{\alpha}: \alpha^{-1} \mathrm{GL}_{1}(A) \rightarrow \mathrm{GL}_{1}(A)$ is a weak equivalence. A log symmetric ring spectrum is a commutative symmetric ring spectrum with a log 
structure. The log symmetric ring spectra generate a full subcategory, denoted $\log (S)$, of $\operatorname{Pre} \mathcal{L} \operatorname{og}(S)$.

A based pre-log structure $(N, \alpha)$ on $A$ is a based log structure if the underlying (unbased) pre-log structure is a $\log$ structure.

Remark 7.5 It might seem more natural to define based log structures in terms of a pullback square in the category of commutative based $\mathcal{I}$-space monoids. If we replace $\mathrm{GL}_{1}(A)$ by $\mathrm{GL}_{1}(A)_{+}$, by adding a disjoint zero, the extended map $\iota_{+}: \mathrm{GL}_{1}(A)_{+} \rightarrow$ $\Omega_{\otimes}^{\bullet} A$ will usually not be a fibration, and the pullback ceases to be homotopy invariant. If we take the homotopy pullback, or equivalently, replace the disjoint zero by the path space of the zero-th component, then it appears that the resulting space of based log derivations (see Definition 11.8) will not be a corepresentable functor, so that we get no good notion of based $\log$ differentials. If we add the full path component of zero in $\Omega_{\otimes}^{\bullet} A$ to $\mathrm{GL}_{1}(A)$, then the $\log$ condition also normalizes the part of $M$ mapping by $\alpha$ to the zero-component, which is undesirable in some topological applications (see Example 7.18).

Definition 7.6 To each pre-log structure $(M, \alpha)$ on $A$ there is an associated log structure $(M, \alpha)^{a}=\left(M^{a}, \alpha^{a}\right)$, where $M^{a}$ is defined by the upper left hand pushout square in the diagram

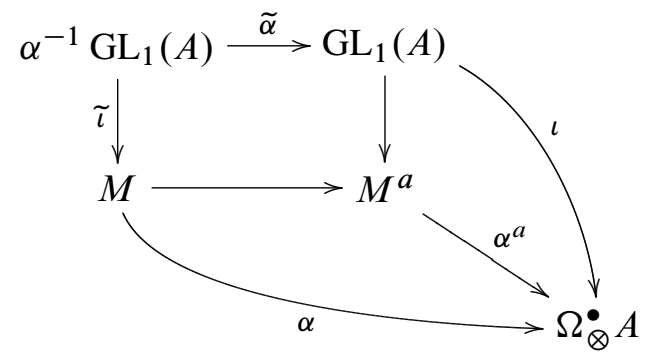

of commutative $\mathcal{I}$-space monoids, and $\alpha^{a}: M^{a} \rightarrow \Omega_{\otimes}^{\bullet} A$ is the canonical map induced by $\alpha$ and $\iota$. When $\alpha^{-1} \mathrm{GL}_{1}(A)$ is trivial, the pushout is the coproduct $M^{a} \cong$ $M \otimes \mathrm{GL}_{1}(A)$, which is weakly equivalent to the cartesian product $M \times \mathrm{GL}_{1}(A)$ (for cofibrant and semistable $M$ and $A$ ).

Lemma 7.7 The associated $\log$ structure $\left(M^{a}, \alpha^{a}\right)$ is a $\log$ structure on $A$. If $(A, M, \alpha)$ is a cofibrant $\log$ symmetric ring spectrum, the canonical map $(A, M, \alpha) \rightarrow$ $\left(A, M^{a}, \alpha^{a}\right)$ is a cofibration and a weak equivalence.

Proof A product in $M^{a}$ maps to a homotopy unit in $\Omega_{\otimes}^{\bullet} A$ if and only if each factor maps to a homotopy unit. Hence the preimage $\left(\alpha^{a}\right)^{-1} \mathrm{GL}_{1}(A) \subset M^{a}$ is the pushout 
of the preimages

$$
\alpha^{-1} \mathrm{GL}_{1}(A) \stackrel{\text { id }}{\leftarrow} \alpha^{-1} \mathrm{GL}_{1}(A) \stackrel{\widetilde{\alpha}}{\rightarrow} \mathrm{GL}_{1}(A)
$$

and it is therefore isomorphic to $\mathrm{GL}_{1}(A)$.

If $\Omega_{\otimes}^{\bullet} A$ is obtained by attaching $\mathcal{C} \mathcal{S}^{\mathcal{I}}$-cells of the form $\left(C \Delta^{n}, C \partial \Delta^{n}\right)$ to $M$, then each cell either lies within $\mathrm{GL}_{1}(A)$, or meets $\mathrm{GL}_{1}(A)$ only at the monoid unit. Hence $\mathrm{GL}_{1}(A)$ is obtained from $\alpha^{-1} \mathrm{GL}_{1}(A)$ by attaching the cells of the first kind, only, so $\tilde{\alpha}$ is a cofibration. Hence the pushout defining $M^{a}$ is homotopically meaningful when $(A, M, \alpha)$ is cofibrant.

If, furthermore, $(M, \alpha)$ is a $\log$ structure on $A$, then $\tilde{\alpha}$ is a cofibration and a weak equivalence, so its pushout $M \rightarrow M^{a}$ is also a cofibration and a weak equivalence.

Lemma 7.8 The logification functor $(-)^{a}: \operatorname{Pr} \operatorname{L} \log (S) \rightarrow \mathcal{L} \log (S)$ induces a left adjoint to the forgetful functor, at the level of homotopy categories. There is a natural chain of weak equivalences

$$
\begin{aligned}
\mathcal{L} \operatorname{og}(S)\left(\left(A, M^{a}\right),(B, N)\right) & \simeq \operatorname{Pre} \mathcal{L} \operatorname{og}(S)((A, M),(B, N)) \\
& \simeq \operatorname{Pre} \mathcal{L} \log (S)\left((A, M),\left(B, N^{a}\right)\right)
\end{aligned}
$$

for $(A, M)$ a pre-log symmetric ring spectrum and $(B, N)$ a log symmetric ring spectrum.

Proof Given a map $\left(f, f^{b}\right):(A, M, \alpha) \rightarrow(B, N, \beta)$ of pre-log symmetric ring spectra, where $(N, \beta)$ is a $\log$ structure on $B$, we get a commutative diagram

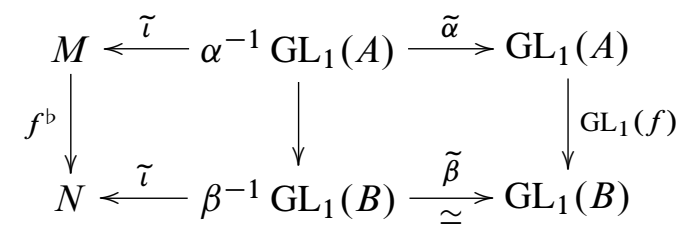

and a chain of maps

$$
M^{a} \stackrel{f^{b, a}}{\longrightarrow} N^{a} \longleftarrow N
$$

When $(B, N, \beta)$ is cofibrant, the right hand map is a weak equivalence. Hence we get a well-defined right adjoint morphism $\left(f, f^{b, a}\right):\left(A, M^{a}\right) \rightarrow(B, N)$, in the homotopy category. 
Definition 7.9 To each based pre-log structure $(N, \alpha)$ on $A$ we associate an associated based log structure $(N, \alpha)^{a}=\left(N^{a}, \alpha^{a}\right)$, where $N^{a}$ is defined by the pushout square

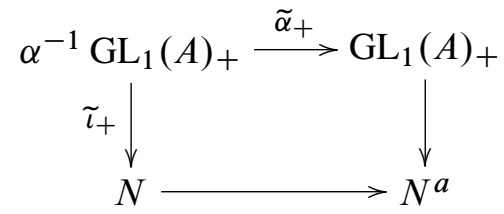

of commutative based $\mathcal{I}$-space monoids. The map $\alpha^{a}: N^{a} \rightarrow \Omega_{\otimes}^{\bullet} A$ is the pushout of the maps $\alpha$ and $\iota_{+}: \mathrm{GL}_{1}(A)_{+} \rightarrow \Omega_{\otimes}^{\bullet} A$. When $\alpha^{-1} \mathrm{GL}_{1}(A)$ is trivial the pushout is the coproduct $N^{a}=N \boxminus \mathrm{GL}_{1}(A)_{+}$, which for reasonable $N$ and $A$ is weakly equivalent to $N \wedge \mathrm{GL}_{1}(A)_{+}$. The analogues of Lemmas 7.7 and 7.8 hold for based $\log$ structures.

Definition 7.10 Let $A$ be a commutative symmetric ring spectrum. The trivial pre-log structure on $A$ is the pair $(\{1\}, \alpha)$, where $\{1\}$ is the initial and terminal commutative $\mathcal{I}$-space monoid, and $\alpha:\{1\} \rightarrow \Omega_{\otimes}^{\bullet} A$ is the unique map. The trivial log structure on $A$ is the associated $\log$ structure $(\{1\}, \alpha)^{a}=\left(\mathrm{GL}_{1}(A), \iota\right)$. We say that $\left(A, \mathrm{GL}_{1}(A), \iota\right)$ is a trivial log symmetric ring spectrum. We get functors $(-)^{\text {triv }}: \mathcal{C} \mathcal{S} p^{\Sigma} \rightarrow \operatorname{Pre} \log (S)$ and $(-)^{\text {triv }, a}: \mathcal{C S} p^{\Sigma} \rightarrow \log (S)$, left adjoint to the forgetful functors.

Remark 7.11 We view the opposite category $\log (S)^{\text {op }}$ as the category of affine derived log schemes, with a forgetful functor to the category $\mathcal{A} f f(S)=\left(\mathcal{C S} p^{\Sigma}\right)^{\text {op }}$ of affine derived schemes, in the sense of Jacob Lurie. It is no more difficult to formulate the global notion of a derived log scheme, which is locally glued together from affine derived log schemes, than it is to define derived classical schemes in terms of affine derived schemes. We will only work locally, ie, on affine pieces, in this paper.

Definition 7.12 Let $M$ be a commutative monoid in $\mathcal{I}$-spaces. The canonical pre-log structure on $S[M]$ is the pair $(M, \zeta)$, where $\zeta: M \rightarrow \Omega_{\otimes}^{\bullet} S[M]$ is right adjoint to the identity on $S[M]$. The canonical log structure on $S[M]$ is the associated log structure $(M, \zeta)^{a}$.

Let $N$ be a commutative monoid in based $\mathcal{I}$-spaces. The canonical based pre-log structure on $\Sigma^{\bullet} N$ is the pair $(N, \zeta)$, where $\zeta: N \rightarrow \Omega_{\otimes}^{\bullet} \Sigma^{\bullet} N$ is right adjoint to the identity on $\Sigma^{\bullet} N$. The canonical based log structure on $\Sigma^{\bullet} N$ is the associated based $\log$ structure $(N, \zeta)^{a}$.

We get free functors $(-)^{\text {can }}: \mathcal{C} \mathcal{S}^{\mathcal{I}} \rightarrow \operatorname{Pre} \mathcal{L} o g(S),(-)^{\text {can }}: \mathcal{C S}_{0}^{\mathcal{I}} \rightarrow \operatorname{Pre} \mathcal{L}_{0} g_{0}(S)$, $(-)^{\text {can }, a}: \mathcal{C S}^{\mathcal{I}} \rightarrow \mathcal{L} \operatorname{og}(S)$ and $(-)^{\text {can }, a}: \mathcal{C S}_{0}^{\mathcal{I}} \rightarrow \mathcal{L} \log _{0}(S)$, left adjoint to the forgetful functors. 
Lemma 7.13 The functor $(-)_{+}: \operatorname{Pre} \mathcal{L} o g(S) \rightarrow \operatorname{Pre} \log _{0}(S)$, taking $(A, M)$ to $\left(A, M_{+}\right)$, and its restriction $(-)_{+}: \mathcal{L} \operatorname{og}(S) \rightarrow \mathcal{L}_{o} g_{0}(S)$, are left adjoint to the respective forgetful functors.

Remark 7.14 We can summarize these adjunctions in the following diagram, where the $\infty$-symbols indicates an adjunction only in an infinity-categorical sense. As usual, the left adjoints are either on the left hand side, or on top.

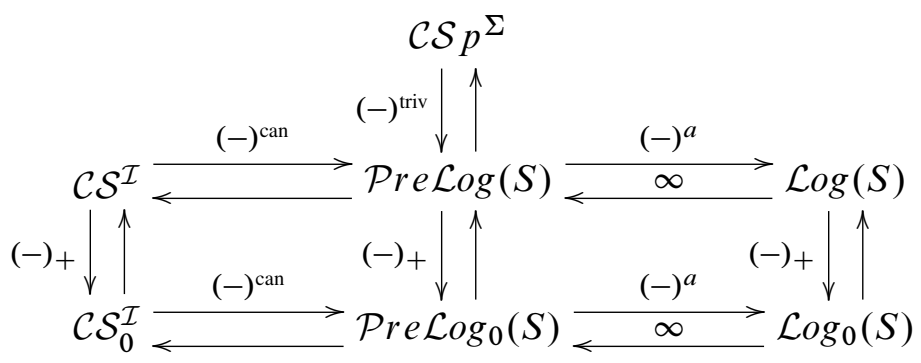

The unlabeled arrows are forgetful functors.

Definition 7.15 For a pre-log symmetric ring spectrum $(A, M)$, the trivial locus is the pre-log symmetric ring spectrum $\left(A\left[M^{-1}\right], \Gamma M\right)$, where

$$
A\left[M^{-1}\right]=A \wedge S[M] S[\Gamma M] .
$$

There is a canonical map $(A, M) \rightarrow\left(A\left[M^{-1}\right], \Gamma M\right)$, and $\left(A\left[M^{-1}\right], \Gamma M\right)^{a}$ is the trivial $\log$ structure. For $\log$ symmetric ring spectra $(A, M)$ the functor $(A, M) \mapsto$ $A\left[M^{-1}\right]$ is left adjoint to $(-)^{\text {triv, } a}$ (at the level of homotopy- or infinity-categories), which therefore has both a left and a right adjoint.

Example 7.16 Let $(A, M, \alpha)$ be a (discrete) pre-log ring, and let $c: \mathcal{C M}$ on $\rightarrow \mathcal{C S}^{\mathcal{I}}$ be the "constant $\mathcal{I}$-space" functor that views a commutative monoid as a commutative $\mathcal{I}$ space monoid. The Eilenberg-Mac Lane symmetric ring spectrum $H A$ has $n$-th space $H A_{n} \simeq K(A, n)$, and there is a natural equivalence $c:(A, \cdot) \rightarrow \Omega^{\bullet} H A$ of commutative $\mathcal{I}$-space monoids. Furthermore, there is a natural equivalence $c: \mathrm{GL}_{1}(A) \rightarrow \mathrm{GL}_{1}(H A)$, and $c$ commutes with pullbacks. Hence $(H A, c M, c \alpha)$ is a pre-log symmetric ring spectrum, and it is a log symmetric spectrum if and only if $(A, M, \alpha)$ is a log ring. We usually write $(H A, M, \alpha)$ in place of $(H A, c M, c \alpha)$.

Example 7.17 Let $A$ be a commutative $S$-algebra, and let $Y \in \mathcal{T}$ be a based space. Choose a based map $y: Y \rightarrow \Omega_{\otimes}^{\infty} A$, and extend $y$ freely to a map $\bar{y}_{0}: L_{0} Y \rightarrow \Omega_{\otimes}^{\infty} A$ of $\mathcal{L}_{0}$-spaces ( $=E_{\infty}$ spaces with zero). Then $\left(A, L_{0} Y, \bar{y}_{0}\right)$ is a based pre-log $S-$ algebra. We call $\left(L_{0} Y, \bar{y}_{0}\right)$ the free $E_{\infty}$ based pre-log structure on $A$ generated by $y$. 
When $X \in \mathcal{U}$ is an unbased space, $x: X \rightarrow \Omega_{\otimes}^{\infty} A$ an unbased map, and $\bar{x}: L X \rightarrow \Omega_{\otimes}^{\infty} A$ its free extension to a map of $\mathcal{L}$-spaces ( $=E_{\infty}$ spaces), we get the free $E_{\infty}$ (unbased) pre-log structure $(L X, \bar{x})$ on $A$ generated by $x$.

When $Y=X_{+}$has a disjoint base point, so $L_{0} Y=(L X)_{+}$, the free $E_{\infty}$ based pre-log structure generated by $y: Y \rightarrow \Omega_{\otimes}^{\infty} A$ restricts to the free $E_{\infty}$ unbased pre-log structure generated by $x: X \rightarrow \Omega_{\otimes}^{\infty} A$, where $x=y \mid X$. When the base point of $Y$ is not isolated, there is no such overlap of definitions.

Example 7.18 As special cases of the previous example, we consider the commutative $S$-algebras $A=\ell$ and $B=k u$ or $k u_{(p)}$. Here $k u$ is the connective complex $K-$ theory spectrum, with $\Omega^{\infty} k u \simeq B U \times \mathbb{Z}$ and $\pi_{*} k u=\mathbb{Z}[u]$ with $|u|=2$. For a fixed odd prime $p, \ell$ is the Adams summand of the $p$-local $K$-theory spectrum $k u_{(p)}$, with $\Omega^{\infty} \ell \simeq W \times \mathbb{Z}_{(p)}$ and $\pi_{*} \ell=\mathbb{Z}_{(p)}\left[v_{1}\right]$ with $\left|v_{1}\right|=q=2 p-2$.

We have based and unbased pre-log structures $\left(L_{0} S^{2}, \bar{u}_{0}\right)$ and $\left(L S^{2}, \bar{u}\right)$ on $k u$, generated by a map $S^{2} \rightarrow \Omega_{\otimes}^{\infty} k u$ representing $u \in \pi_{2} k u$. Similarly, we have based and unbased pre-log structures $\left(L_{0} S^{q}, \bar{v}_{1,0}\right)$ and $\left(L S^{q}, \bar{v}_{1}\right)$ on $\ell$, generated by a map $S^{q} \rightarrow \Omega_{\otimes}^{\infty} \ell$ representing $v_{1} \in \pi_{q} \ell$. Here $L_{0} S^{d}=\bigvee_{j \geq 0} \mathcal{L}(j)_{+} \wedge_{\Sigma_{j}} S^{d j}$ and $L S^{d}=\bigsqcup_{j \geq 0} \mathcal{L}(j) \times_{\Sigma j}\left(S^{d}\right)^{j}$. Note that these pre-log structures map entirely into the zero-component $B U \cong B U \times\{0\}$ (resp. $W \cong W \times\{0\}$ ), with the single exception that the $E_{\infty}$ space unit in the $j=0$ summand of the source maps to the $E_{\infty}$ space unit 1 in $B U \times\{1\}$ (resp. $W \times\{1\}$ ).

There is a map $f: \ell \rightarrow k u_{(p)}$ of commutative $S$-algebras, inducing the ring homomorphism $f_{*}: \mathbb{Z}_{(p)}\left[v_{1}\right] \rightarrow \mathbb{Z}_{(p)}[u]$ that takes $v_{1}$ to $u^{p-1}$. In the based category, this lifts to a map

$$
\left(f, f^{b}\right):\left(\ell, L_{0} S^{q}, \bar{v}_{1,0}\right) \rightarrow\left(k u_{(p)}, L_{0} S^{2}, \bar{u}_{0}\right)
$$

of based pre-log $S$-algebras, where $f^{b}: L_{0} S^{q} \rightarrow L_{0} S^{2}$ is freely generated by the composite map $S^{q} \cong\left(S^{2}\right)^{\wedge(p-1)} \rightarrow \mathcal{L}(p-1)_{+} \wedge_{\Sigma_{p-1}}\left(S^{2}\right)^{\wedge(p-1)} \rightarrow L_{0} S^{2}$. The middle map depends on a contractible choice of a point in $\mathcal{L}(p-1)$. To make the diagram

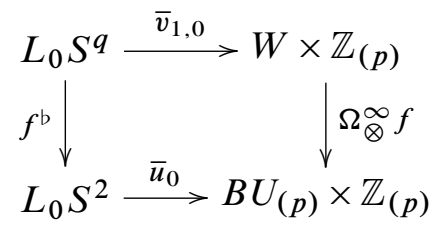

strictly commute, we must assume that the representing map $S^{q} \rightarrow W \times \mathbb{Z}_{(p)}$ for $v_{1}$ is chosen to lift the composite map $S^{q} \rightarrow L_{0} S^{2} \rightarrow B U_{(p)} \times \mathbb{Z}_{(p)}$. See the examples at the end of Section 12 for more on this map of based pre-log $S$-algebras. 
Remark 7.19 In the unbased category, there is no map

$$
\left(f, f^{b}\right):\left(\ell, L S^{q}, \bar{v}_{1}\right) \nrightarrow \rightarrow\left(k u_{(p)}, L S^{2}, \bar{u}\right)
$$

of (unbased) pre-log $S$-algebras lifting the usual map $f: \ell \rightarrow k u_{(p)}$, for odd primes $p$. For $f^{\text {b }}: L S^{q} \rightarrow L S^{2}$ must freely extend a map

$$
S^{q} \rightarrow L S^{2}=\coprod_{j \geq 0} \mathcal{L}(j) \times \Sigma_{j}\left(S^{2}\right)^{j}
$$

that takes $S^{q}$ to the $j=p-1$ summand in a rationally nontrivial way. But any map from $S^{q}$ to $\mathcal{L}(j) \times_{\Sigma_{j}}\left(S^{2}\right)^{j}$ lifts through $\mathcal{L}(j) \times\left(S^{2}\right)^{j} \simeq\left(S^{2}\right)^{j}$, and $\pi_{q}\left(\left(S^{2}\right)^{j}\right)$ is torsion.

This is an unsatisfactory feature of the unbased theory, since we expect $f: \ell \rightarrow k u_{(p)}$ to behave as a tamely ramified extension of commutative $S$-algebras, with ramification locus corresponding to $\left(v_{1}\right) \subset \pi_{*} \ell$ downstairs, and $(u) \subset \pi_{*} k u_{(p)}$ upstairs. The ramification should be tame, since $\left(v_{1}\right)=(u)^{p-1}$ and the ramification index $e=p-1$ is prime to the residue characteristic. By analogy with Example 4.32, we might therefore expect there to be $\log$ structures on $\ell$ and $k u_{(p)}$ such that $f$ lifts to a log étale map. Further evidence in this direction is given by Christian Ausoni's discussion in [4, Section 10]. As Example 7.18 and this remark shows, this is plausible in the context of based log structures, but not so for unbased log structures.

However, as in Example 12.16, the free $E_{\infty}$ based $\log$ structures on $\ell$ and $k u_{(p)}$ are too simple to realize $f$ as part of a log étale map. In a later paper, we will describe a recently found modification of the current theory, working with commutative $M U-$ algebras in place of commutative $S$-algebras, where this log étale realization problem has a positive solution. Here $M U$ is the complex bordism spectrum.

Example 7.20 Among the based pre-log structures $(N, \alpha)$ on a commutative $S$ algebra $A$, such that $\alpha$ takes all but the identity element of $A$ to the zero-th path component of $A$, there is a terminal example. It has $N=\left(\Omega_{0}^{\bullet} A\right) \sqcup\{1\}$, where $\Omega_{0}^{\bullet} A \subset \Omega_{\otimes}^{\bullet} A$ denotes the full path component of the base point 0 . Note that $N$ has the multiplicative $E_{\infty}$ structure, not the additive one. This full zero-th path component pre-log structure is canonically associated to $A$, and each map like $f: \ell \rightarrow k u_{(p)}$ of commutative $S$-algebras is covered by a corresponding pre-log map. However, it seems to be difficult to determine the associated based deloopings $B_{\wedge}^{n}(N)$, and we have not been able to analyze any interesting cases.

Example 7.21 Let $A$ be a commutative symmetric ring spectrum, and let $Y$ be a based $\mathcal{I}$-space. Choose a based $\mathcal{I}$-space map $y: Y \rightarrow \Omega_{\otimes}^{\bullet} A$, and extend $y$ freely 
to a map $\bar{y}_{0}: C_{0} Y \rightarrow \Omega_{\otimes}^{\bullet} A$. Then $\left(A, C_{0} Y, \bar{y}_{0}\right)$ is a based pre-log symmetric ring spectrum. A homotopy class $[u]$ in $\pi_{d}(A)$, for $d \geq 0$, is realized at some level $n$ in the $\mathcal{I}$-space $\Omega_{\otimes}^{\bullet} A$, by a map $u: S^{d} \rightarrow \Omega^{n} A_{n}$. When $A$ is positively fibrant, we may assume $n=1$. Letting $Y=F_{n} S^{d}$ be the free $\mathcal{I}$-space generated by $S^{d}$ at level $n$, we get an $\mathcal{I}$-space map $y: Y=F_{n} S^{d} \rightarrow \Omega_{\otimes}^{\bullet} A$, which generates a based pre-log structure $\left(C_{0} Y, \bar{y}_{0}\right)$ on $A$, as above. We call this the free commutative pre-log structure on $A$ generated by $u$. There is, of course, a corresponding unbased construction.

Definition 7.22 Let $f: A \rightarrow B$ be a map of commutative symmetric ring spectra, and let $(M, \alpha)$ be a pre-log structure on $A$. The inverse image log structure

$$
\left(f^{*} M, f^{*} \alpha\right)=\left(M, \Omega_{\otimes}^{\bullet} f \circ \alpha\right)^{a}
$$

on $B$ is the $\log$ structure associated to the pre-log structure given by the composite map

$$
M \stackrel{\alpha}{\rightarrow} \Omega_{\otimes}^{\bullet} A \stackrel{\Omega_{\otimes}^{\bullet} f}{\longrightarrow} \Omega_{\otimes}^{\bullet} B
$$

of commutative $\mathcal{I}$-space monoids. Hence there is a commutative diagram

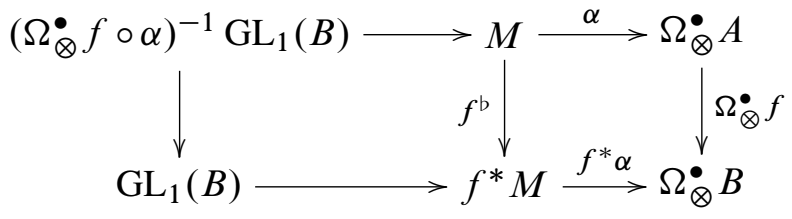

where the left hand square is a pushout square. In particular, there is a canonical map $\left(f, f^{b}\right):(A, M) \rightarrow\left(B, f^{*} M\right)$ of (pre-)log symmetric ring spectra.

Similar definitions can be made for based (pre-)log structures, using the associated based log structure from Definition 7.9.

Lemma 7.23 The space of $\log$ maps $(A, M) \rightarrow(B, N)$ covering a fixed map $f: A \rightarrow B$ of commutative symmetric ring spectra is weakly equivalent to the space of log maps $\left(B, f^{*} M\right) \rightarrow(B, N)$ covering id $_{B}$, the identity on $B$.

Proof The space of commutative $\mathcal{I}$-space monoid maps $M \rightarrow N$ that make the following diagram commute

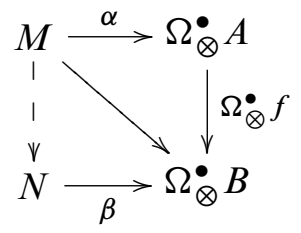


agrees with the space of pre-log maps from $\left(M, \Omega_{\otimes}^{\bullet} f \circ \alpha\right)$ to $(N, \beta)$ covering $\mathrm{id}_{B}$, and this is weakly equivalent to the space of $\log$ maps from $\left(M, f^{*} \alpha\right)$ to $(N, \beta)$ covering $\operatorname{id}_{B}$, essentially by Lemma 7.8 .

Lemma 7.24 The canonical map $(M, \alpha) \rightarrow\left(M^{a}, \alpha^{a}\right)$ from a pre-log structure on $A$ to the associated log structure induces a weak equivalence

$$
\left(f^{*} M, f^{*} \alpha\right) \stackrel{\simeq}{\rightarrow}\left(f^{*}\left(M^{a}\right), f^{*}\left(\alpha^{a}\right)\right)
$$

of inverse image log structures on $B$.

Proof The part $\left(\Omega_{\otimes}^{\bullet} f \circ \alpha^{a}\right)^{-1} \mathrm{GL}_{1}(B)$ of the pushout $M^{a}$ that sits over $\mathrm{GL}_{1}(B)$ is the pushout of the parts of $M \leftarrow \alpha^{-1} \mathrm{GL}_{1}(A) \rightarrow \mathrm{GL}_{1}(A)$ that sit over $\mathrm{GL}_{1}(B)$, ie, the pushout of $\left(\Omega_{\otimes}^{\bullet} f \circ \alpha\right)^{-1} \mathrm{GL}_{1}(B) \leftarrow \alpha^{-1} \mathrm{GL}_{1}(A) \rightarrow \mathrm{GL}_{1}(A)$. So in the commutative diagram

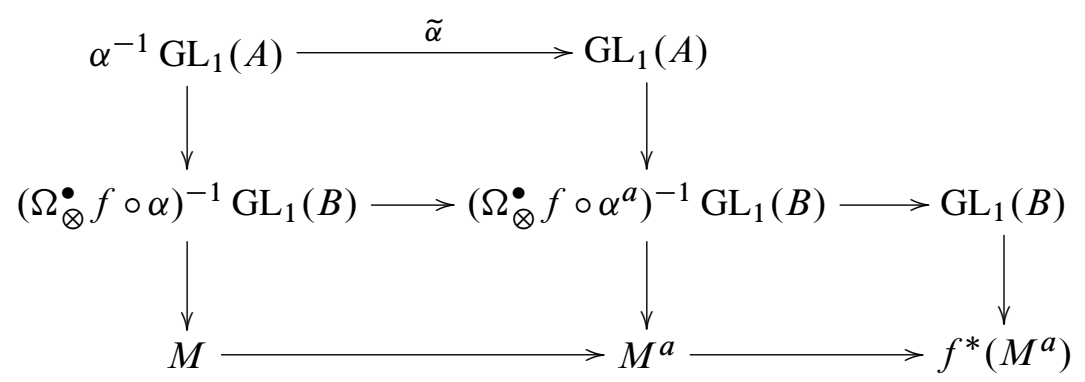

the upper left hand square, the rectangle formed by the two left hand squares, and the lower right hand square are pushout squares. It follows that the lower left hand square, and the rectangle formed by the two lower squares, are pushout squares. Hence $f^{*} M$, which is the pushout of the latter rectangle, is equivalent to $f^{*}\left(M^{a}\right)$.

Definition 7.25 A map $\left(f, f^{b}\right):(A, M) \rightarrow(B, N)$ of log symmetric ring spectra is strict if the corresponding commutative $\mathcal{I}$-space monoid map $f^{*} M \rightarrow N$ is a weak equivalence. We write $\operatorname{str} \log (S) \subset \mathcal{L} \log (S)$ for the subcategory of strict maps.

Definition 7.26 Let $f: A \rightarrow B$ be a map of commutative symmetric ring spectra and let $(N, \beta)$ be a pre-log structure on $B$. The direct image pre-log structure $\left(f_{*} N, f_{*} \beta\right)$ on $A$ is defined by the pullback square

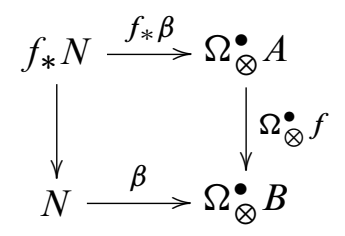


of commutative $\mathcal{I}$-space monoids. When $(N, \beta)$ is a log structure on $B,\left(f_{*} N, f_{*} \beta\right)$ will also be a $\log$ structure on $A$, called the direct image log structure, since the part of $f_{*} N$ sitting over $\mathrm{GL}_{1}(A) \subset \Omega_{\otimes}^{\bullet} A$ is the pullback of $\beta^{-1} \mathrm{GL}_{1} B$ and $\mathrm{GL}_{1}(A)$ over $\mathrm{GL}_{1}(B)$, which then is isomorphic to $\mathrm{GL}_{1}(A)$. There is a canonical map $\left(f, f^{b}\right):\left(A, f_{*} N\right) \rightarrow(B, N)$ of (pre-)log symmetric ring spectra.

Lemma 7.27 The space of $\log$ maps $(A, M) \rightarrow(B, N)$ covering a fixed map $f: A \rightarrow B$ of commutative symmetric ring spectra is weakly equivalent to the space of log maps $(A, M) \rightarrow\left(A, f_{*} N\right)$ covering id $_{A}$, the identity on $A$.

Proof The space of commutative $\mathcal{I}$-space monoid maps $M \rightarrow f_{*} N$ that make the upper square of the diagram

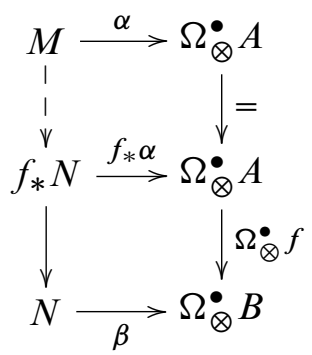

commute agrees, by the universal property of pullbacks, with the space of commutative $\mathcal{I}$-space monoid maps $M \rightarrow N$ that make the outer rectangle commute.

Remark 7.28 The present definition of a pre-log structure on a commutative $S$ algebra $A$ is only really suitable for connective $A$, since the functors $\Omega_{\otimes}^{\infty}$ and $\mathrm{GL}_{1}$ ignore the negative homotopy groups of $A$. In other words, the pre-log structures on $A$ are the same as the pre-log structures on its connective cover, and this is undesirable in some topological applications. For example, the based Bott pre-log structure $\left(L_{0} S^{2}, \bar{u}_{0}\right)$ on the connective $K$-theory spectrum $k u$ is generated by a map $u: S^{2} \rightarrow \Omega_{\otimes}^{\infty} k u$. It gives a nontrivial log structure on $k u$, since multiplication by $u$ induces a map $\Sigma^{2} k u \rightarrow k u$ that is not a weak equivalence. However, the corresponding pre-log structure $\left(L_{0} S^{2}, \Omega_{\otimes}^{\infty} i \circ \bar{u}_{0}\right)$ on the periodic $K$-theory spectrum $K U$, where $i: k u \rightarrow K U$ is the connective covering map, should ideally be viewed as a trivial log structure, since multiplication by $u$ induces a weak equivalence $\Sigma^{2} K U \rightarrow K U$.

We hope to resolve this point in a later paper, as an application of the graded version of $\mathcal{I}$-spaces developed in Sagave-Schlichtkrull [67]. Conversely, the Bott structure on $k u$ should arise as the direct image $i_{*} \mathrm{GL}_{1}(K U)$ of the trivial "graded" log structure on $K U$. More generally, the connective cover $e$ of any commutative $S$-algebra $E$ with periodic homotopy groups should inherit a nontrivial canonical graded log structure $i_{*} \mathrm{GL}_{1}(E)$ from $E$, as the direct image along the connective covering map $i: e \rightarrow E$. 


\section{Logarithmic topological Hochschild homology}

Definition 8.1 A map $\epsilon: M \rightarrow P$ of commutative $\mathcal{I}$-space monoids is exact if the diagram

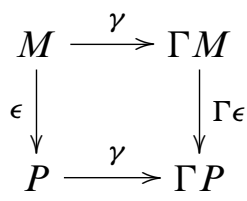

is a homotopy pullback square. A map $\epsilon: M \rightarrow P$ of commutative $\mathcal{I}$-space monoids is virtually surjective if the induced homomorphism $\pi_{0} \Gamma \epsilon: \pi_{0} \Gamma M \rightarrow \pi_{0} \Gamma P$ of abelian groups is surjective. Let

$$
\left(\mathcal{C S}^{\mathcal{I}} / P\right)^{\text {vsur }} \subset \mathcal{C S}^{\mathcal{I}} / P
$$

be the full subcategory of virtually surjective $M$ over $P$. We say that a virtually surjective $M$ over $P$ is replete if it is also exact, ie, if the diagram above is a homotopy pullback square. Let

$$
\left(\mathcal{C S}^{\mathcal{I}} / P\right)^{\mathrm{rep}} \subset\left(\mathcal{C S}^{\mathcal{I}} / P\right)^{\text {vsur }}
$$

be the full subcategory of replete $M$ over $P$.

Definition 8.2 For a virtually surjective $\epsilon: M \rightarrow P$, let the repletion of $M$ over $P$ be the pullback

$$
M^{\text {rep }}=P \times_{\Gamma P} \Gamma M
$$

in the square diagram above, with the canonical structure map $\epsilon^{\mathrm{rep}}: M^{\mathrm{rep}} \rightarrow P$. The following diagram of commutative $\mathcal{I}$-space monoids commutes, where the right hand square is a pullback by construction:

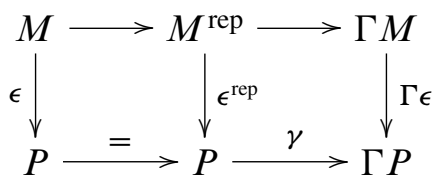

We call the map $M \rightarrow M^{\text {rep }}$ the repletion map.

Proposition 8.3 For virtually surjective $\epsilon: M \rightarrow P$, the maps

$$
M \rightarrow M^{\text {rep }} \rightarrow \Gamma M
$$

induce weak equivalences

$$
\Gamma M \stackrel{\simeq}{\rightarrow} \Gamma\left(M^{\text {rep }}\right) \stackrel{\simeq}{\longrightarrow} \Gamma(\Gamma M)
$$

upon group completion. Hence $M^{\text {rep }}$ is replete over $P$. 
Proof We start with the pullback square defining $M^{\text {rep }}$, from Definition 8.2. For each $q \geq 0$, the square of $q$-fold $\otimes$-products

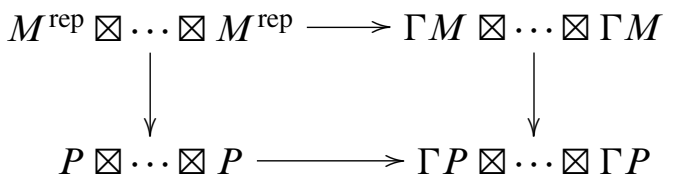

is a homotopy pullback square, since $(P \otimes P)_{h \mathcal{I}} \simeq P_{h \mathcal{I}} \times P_{h \mathcal{I}}$, and similarly in the three other corners of the square. More precisely, this equivalence holds if $P$ is a cofibrant $\mathcal{I}$-space, and similarly for the three other corners, so we should first apply cofibrant replacement to the pullback square defining $M^{\text {rep }}$. This does not affect the homotopy type of $M^{\mathrm{rep}}, \Gamma M$, etc., and will therefore be suppressed in the rest of the argument.

We now wish to apply the Bousfield-Friedlander theorem [16, Theorem B.4], to conclude that the diagonalized square

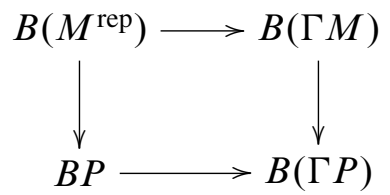

is a homotopy pullback square of commutative $\mathcal{I}$-space monoids. Assuming this, we can pass to pointwise loop spaces to get the homotopy pullback square

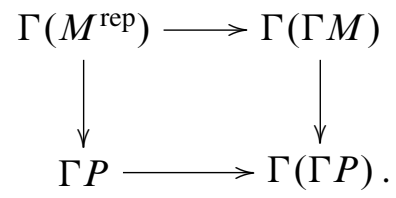

Here the lower horizontal map is a weak equivalence, hence so is the upper horizontal map. It follows that

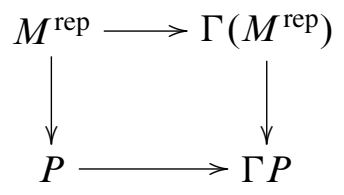

is a homotopy pullback square, so $M^{\text {rep }}$ is replete.

To apply the Bousfield-Friedlander theorem, we need to know that

$$
X_{\bullet}:[q] \mapsto \Gamma M \otimes \cdots \otimes \Gamma M
$$


and $Y_{\bullet}:[q] \mapsto \Gamma P \otimes \cdots \otimes \Gamma P\left(q\right.$ copies of $\Gamma M$, resp. $\Gamma P$ ) satisfy the $\pi_{*}-$ Kan condition (see Bousfield-Friedlander [16, Section B.3]), and that $\pi_{0}^{v}\left(X_{\bullet}\right) \rightarrow \pi_{0}^{v}\left(Y_{\bullet}\right)$ is a (Kan) fibration. The bar construction on any group (or groupoid) is fibrant, and the same argument shows that $X_{\bullet}$ and $Y_{\bullet}$ satisfy the $\pi_{*}-$ Kan condition. The zero-th vertical homotopy groups of $X_{\bullet}$ and $Y_{\bullet}$ are

$$
\pi_{0}^{v}\left(X_{\bullet}\right):[q] \mapsto \pi_{0}\left(X_{q}\right)=\left(\pi_{0} \Gamma M\right)^{q}
$$

and $\pi_{0}^{v}\left(Y_{\bullet}\right)=\left(\pi_{0} \Gamma P\right)^{q}$, so $\pi_{0}^{v}\left(X_{\bullet}\right) \rightarrow \pi_{0}^{v}\left(Y_{\bullet}\right)$ is the map of bar constructions $B\left(\pi_{0} \Gamma M\right) \rightarrow B\left(\pi_{0} \Gamma P\right)$ induced by the group homomorphism $\pi_{0} \Gamma \epsilon: \pi_{0} \Gamma M \rightarrow$ $\pi_{0} \Gamma P$. By assumption $\epsilon: M \rightarrow P$ is virtually surjective, so $\pi_{0} \Gamma \epsilon$ is surjective, and this precisely ensures that $B\left(\pi_{0} \Gamma M\right) \rightarrow B\left(\pi_{0} \Gamma P\right)$ is a fibration.

Lemma 8.4 The functor $(-)^{\text {rep }}\left(\mathcal{C S}^{\mathcal{I}} / P\right)^{\text {vsur }} \rightarrow\left(\mathcal{C S} \mathcal{S}^{\mathcal{I}} / P\right)^{\text {rep }}$ is left adjoint to the forgetful functor, at the level of homotopy- or infinity-categories. (Homotopy) colimits of nonempty diagrams exist in $\left(\mathcal{C S}^{\mathcal{I}} / P\right)^{\text {vsur }}$, and are formed in $\mathcal{C S}^{\mathcal{I}} / P$. Homotopy colimits of nonempty diagrams exist in $\left(\mathcal{C S}^{\mathcal{I}} / P\right)^{\text {rep }}$, and are constructed by first forming the homotopy colimit in $\left(\mathcal{C S}^{\mathcal{I}} / P\right)^{\mathrm{vsur}}$ and then applying $(-)^{\mathrm{rep}}$.

Definition 8.5 Let $(R, P)$ be a base pre-log symmetric ring spectrum. A pre-log symmetric ring spectrum $(A, M)$ over $(R, P)$ is virtually surjective if the underlying commutative $\mathcal{I}$-space monoid $M$ is virtually surjective over $P$. It is a replete pre-log symmetric ring spectrum if the underlying commutative $\mathcal{I}$-space monoid $M$ is replete over $P$.

Let $(\mathcal{P r e} \mathcal{L} \operatorname{og}(S) /(R, P))^{\text {vsur }}$ and $(\mathcal{P r e} \mathcal{L} \operatorname{og}(S) /(R, P))^{\text {rep }}$ be the full subcategories of $\operatorname{Pre} \mathcal{L} \operatorname{og}(S) /(R, P)$ generated by the virtually surjective and the replete pre-log symmetric ring spectra, respectively. Let

$$
(-)^{\text {rep }}:(\mathcal{P r e} \mathcal{L} \operatorname{og}(S) /(R, P))^{\mathrm{vsur}} \rightarrow(\mathcal{P} r e \mathcal{L} \operatorname{og}(S) /(R, P))^{\text {rep }}
$$

be the functor that takes a virtually surjective $(A, M)$ over $(R, P)$ to the replete pre-log symmetric ring spectrum

$$
(A, M)^{\mathrm{rep}}=\left(A \wedge S[M] S\left[M^{\mathrm{rep}}\right], M^{\mathrm{rep}}\right)
$$

over $(R, P)$.

Homotopy colimits of nonempty diagrams in $(\mathcal{P r e} \mathcal{L} \operatorname{og}(S) /(R, P))^{\text {rep }}$ are constructed by first forming the homotopy colimit in $\operatorname{Pre} \log (S) /(R, P)$, thereby remaining within $(\operatorname{Pre} \mathcal{L} \operatorname{og}(S) /(R, P))^{\mathrm{vsur}}$, and then applying $(-)^{\mathrm{rep}}$.

Lemma 8.6 Let $(A, M, \alpha)$ be a replete pre-log symmetric ring spectrum over a log symmetric ring spectrum $(R, P, \rho)$. Then $F M=\alpha^{-1} \mathrm{GL}_{1}(A)$. 
Proof Consider the diagram

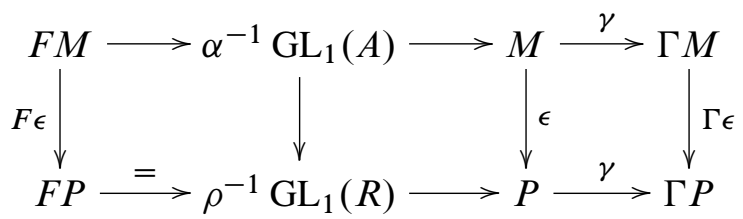

of commutative $\mathcal{I}$-space monoids. The left hand and middle horizontal maps are inclusions of a full set of path components. The homomorphism $\pi_{0} \Gamma \epsilon$ is surjective, the right hand square is a homotopy pullback, and the inclusion $F P \rightarrow \rho^{-1} \mathrm{GL}_{1}(R)$ is the identity. With these modifications, the proof proceeds just like the proof of Lemma 3.13 .

Proposition 8.7 Let $(A, M)$ be a replete pre-log symmetric ring spectrum over a log symmetric ring spectrum $(R, P)$. Then the associated log symmetric ring spectrum $\left(A, M^{a}\right)$ is a replete log symmetric ring spectrum over $(R, P)$.

Proof The proof proceeds like that of Proposition 3.14, except that we do not need to assume that $P$ is "integral" in order to know that

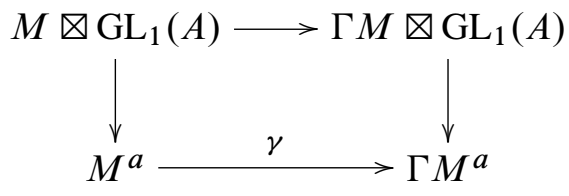

is a homotopy pullback square, since the lower row is obtained by forming the $F M-$ homotopy orbits of the upper row, up to weak equivalence.

Remark 8.8 In joint work with Steffen Sagave we develop a theory of log modules over a log symmetric ring spectrum $(R, P)$, given as the stable model category of spectra in the based category $(R, P) / \log ^{\text {rep }} /(R, P)$ of replete log symmetric ring spectra under and over $(R, P)$.

Example 8.9 Let $(A, M)$ be a pre-log symmetric ring spectrum and $X_{\bullet}$ a simplicial set. The $X_{\bullet}$-fold $\otimes$-product $X_{\bullet} \otimes M$ is the diagonal of the simplicial commutative $\mathcal{I}$-space monoid

$$
[q] \mapsto X_{q} \otimes M=M \otimes \cdots \otimes M
$$

(with one copy of $M$ for each element of $X_{q}$ ). There is a natural weak equivalence $\Gamma\left(X_{\bullet} \otimes M\right) \simeq X_{\bullet} \otimes \Gamma M$. 
Let $Y_{\bullet}$ be a nonempty simplicial set. The $Y_{\bullet}-$ fold replete $\otimes$-product $Y_{\bullet} \otimes^{\text {rep }} M$ over $M$ is the pullback

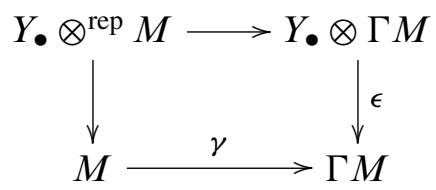

of commutative $\mathcal{I}$-space monoids. If $Y_{\bullet}=\left(X_{\bullet}\right)_{+}$has a disjoint base point, then $Y_{\bullet} \otimes^{\text {rep }} M \simeq M \times(X \bullet \otimes \Gamma M)$. The $Y_{\bullet}$-fold replete smash product $Y_{\bullet} \otimes^{\text {rep }}(A, M)$ is the replete pre-log symmetric $\operatorname{ring} \operatorname{spectrum}\left(Y_{\bullet} \otimes^{\text {rep }} A, Y_{\bullet} \otimes \otimes^{\text {rep }} M\right)$ over $(A, M)$ given by the pushout

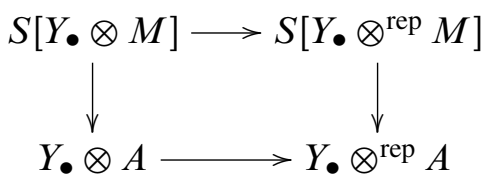

of commutative symmetric ring spectra.

Definition 8.10 Let $M$ be a commutative $\mathcal{I}$-space monoid. The cyclic bar construction on $M$ is the commutative $\mathcal{I}$-space monoid $B^{\text {cy }} M=S_{\bullet}^{1} \otimes M$, where $S_{\bullet}^{1}=\Delta_{\bullet}^{1} / \partial \Delta_{\bullet}^{1}$ and the tensor product is formed in $\left(\mathcal{S}^{\mathcal{I}}, \bigotimes, *\right)$. The replete bar construction $B^{\text {rep }} M=S_{\bullet}^{1} \otimes^{\text {rep }} M$ is the repletion of $B^{\text {cy }} M$ over $M$, given as the pullback

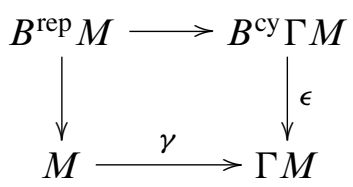

of commutative $\mathcal{I}$-space monoids. Both $\gamma$ and $\epsilon$ are maps of cyclic commutative $\mathcal{I}$-space monoids, where $M$ and $\Gamma M$ have the trivial cyclic structure, so $B^{\text {rep }} M$ is a cyclic commutative $\mathcal{I}$-space monoid, and $B^{\text {cy }} M \rightarrow B^{\text {rep }} M$ is a cyclic map.

There are natural weak equivalences $B^{\text {cy }} \Gamma M \simeq \Gamma M \otimes B(\Gamma M)$ and $B^{\text {rep }} M \simeq$ $M \otimes B M \simeq M \otimes B(\Gamma M)$. The latter equivalence depends on the equivalence $M \times_{\Gamma M}(\Gamma M \otimes B(\Gamma M)) \simeq M \otimes B(\Gamma M)$, which can be seen by using the equivalance $\left(M_{1} \otimes M_{2}\right)_{h \mathcal{I}} \simeq\left(M_{1}\right)_{h \mathcal{I}} \times\left(M_{2}\right)_{h \mathcal{I}}$. The repletion map

$$
B^{\mathrm{cy}} M \rightarrow B^{\mathrm{rep}} M \simeq M \times B(\Gamma M)
$$

is homotopic to the composite

$$
(\epsilon, \pi): B^{\mathrm{cy}} M \stackrel{\Delta}{\rightarrow} B^{\mathrm{cy}} M \times B^{\mathrm{cy}} M \stackrel{\epsilon \times \pi}{\longrightarrow} M \times B M \simeq M \times B(\Gamma M)
$$

where $\epsilon$ is the augmentation and $\pi$ is the usual projection map. 
The topological Hochschild homology of a commutative symmetric ring spectrum $A$ is the commutative $A$-algebra $\operatorname{THH}(A)=S_{\bullet}^{1} \otimes A$. If $A=S[M]$ then

$$
\mathrm{THH}(S[M])=S_{\bullet}^{1} \otimes S[M] \cong S\left[S_{\bullet}^{1} \otimes M\right]=S\left[B^{\mathrm{cy}} M\right] .
$$

Definition 8.11 Let $(A, M, \alpha)$ be a pre-log symmetric ring spectrum. The $\log$ topological Hochschild homology of $(A, M)$, denoted $\operatorname{THH}(A, M)$, is defined to be $S_{\bullet}^{1} \otimes^{\text {rep }}(A, M)$. Hence there is a pushout square

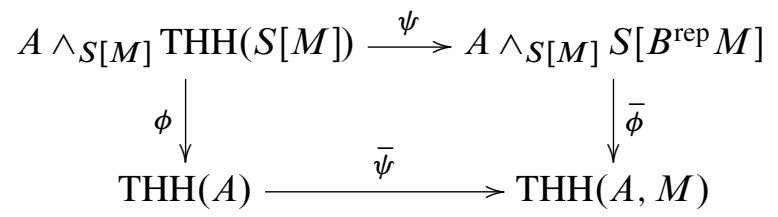

of commutative $A$-algebras. The map $\phi$ is induced by $\bar{\alpha}: S[M] \rightarrow A$, and the map $\psi$ is induced by the repletion map $B^{\text {cy }} M \rightarrow B^{\text {rep }} M$. Both $\phi$ and $\psi$ are maps of cyclic commutative $A$-algebras, so $\operatorname{THH}(A, M)$ is a cyclic commutative $A$-algebra.

Remark 8.12 In view of the identification $\operatorname{THH}(S[M]) \cong S\left[B^{\text {cy }} M\right], \operatorname{THH}(A, M)$ can also be defined by the pushout square

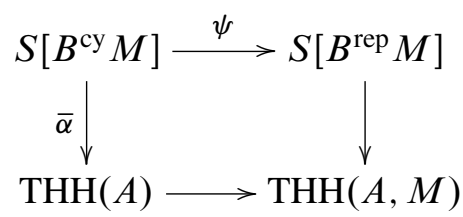

of commutative symmetric ring spectra, where the upper horizontal map $\psi$ is induced by the repletion map.

Example 8.13 Let $A=H \mathbb{Z}_{p}$ be the Eilenberg-Mac Lane spectrum, let $M=\langle p\rangle=$ $\left\{p^{j} \mid j \geq 0\right\}$, and let $\alpha:\langle p\rangle \rightarrow \mathbb{Z}_{p} \simeq \Omega^{\bullet} A$ be the inclusion. Applying base change along $S \rightarrow H \mathbb{Z}_{p} \rightarrow H=H \mathbb{F}_{p}$ to the pushout square of Remark 8.12 we get a pushout square

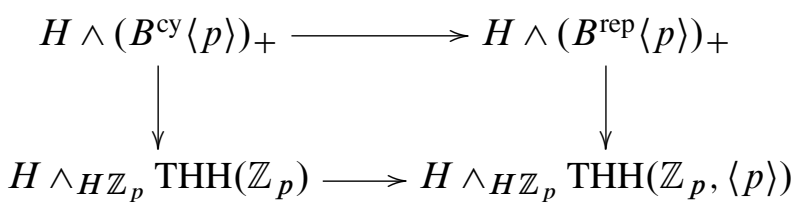

of commutative $H$-algebras. Recall from Propositions 3.20 and 3.21 that

$$
B^{\mathrm{cy}}\langle p\rangle \simeq * \sqcup \coprod_{j \geq 1} S^{1}(j) \quad \text { and } \quad B^{\mathrm{rep}}\langle p\rangle \simeq \coprod_{j \geq 0} S^{1}(j) .
$$


The homotopy algebras in the upper row are

$$
\begin{aligned}
H_{*}\left(B^{\mathrm{cy}}\langle p\rangle\right) & =P(g) \otimes E(d p) \\
H_{*}\left(B^{\text {rep }}\langle p\rangle\right) & =P(g) \otimes E(d \log p)
\end{aligned}
$$

where $g$ is the generator of $H_{0}\left(S^{1}(1)\right)$ that corresponds to the 0 -simplex $(p), d p$ is the generator of $H_{1}\left(S^{1}(1)\right)$ that corresponds to the loop $(1, p)$, and $d \log p$ is the generator of $H_{1}\left(S^{1}(0)\right)$ that corresponds to the loop $\left(p^{-1}, p\right)$. The repletion map induces $g \mapsto g$ and $d p \mapsto g d \log p$. Furthermore,

$$
\pi_{*}\left(H \wedge_{H \mathbb{Z}_{p}} \operatorname{THH}\left(\mathbb{Z}_{p}\right)\right)=\pi_{*}\left(\operatorname{THH}\left(\mathbb{Z}_{p}\right) ; \mathbb{Z} / p\right)=E\left(\lambda_{1}\right) \otimes P\left(\mu_{1}\right)
$$

where $\left|\lambda_{1}\right|=2 p-1$ and $\left|\mu_{1}\right|=2 p$. This calculation is due to Marcel Bökstedt (unpublished, ca. 1987). For a proof close to Bökstedt's original argument, see AngeltveitRognes [2, Theorem 5.12(a)] for $m=1$, using the convention $B P\langle 0\rangle=H \mathbb{Z}_{(p)}$. For an earlier reference, see Franjou-Pirashvili [23].

The map $\bar{\alpha}$ induces $g \mapsto 0$ and $d p \mapsto 0$. Hence the Künneth spectral sequence

$$
\begin{aligned}
E_{* *}^{2} & =\operatorname{Tor}_{* *}^{H_{*}\left(B^{\mathrm{cy}}\langle p\rangle\right)}\left(\pi_{*}\left(\operatorname{THH}\left(\mathbb{Z}_{p}\right) ; \mathbb{Z} / p\right), H_{*}\left(B^{\mathrm{rep}}\langle p\rangle\right)\right) \\
& \Longrightarrow \pi_{*}\left(\operatorname{THH}\left(\mathbb{Z}_{p},\langle p\rangle\right) ; \mathbb{Z} / p\right)
\end{aligned}
$$

has $E^{2}$-term

$$
\begin{aligned}
E_{* *}^{2} & =\operatorname{Tor}_{* *}^{P(g) \otimes E(d p)}\left(E\left(\lambda_{1}\right) \otimes P\left(\mu_{1}\right), P(g) \otimes E(d \log p)\right) \\
& \cong E\left(\lambda_{1}\right) \otimes P\left(\mu_{1}\right) \otimes E(d \log p) \otimes \operatorname{Tor}_{* *}^{E(d p)}\left(\mathbb{F}_{p}, \mathbb{F}_{p}\right) \\
& \cong E\left(d \log p, \lambda_{1}\right) \otimes P\left(\mu_{1}\right) \otimes \Gamma\left(\kappa_{0}\right)
\end{aligned}
$$

where the generators have bidegrees $|d \log p|=(0,1),\left|\lambda_{1}\right|=(0,2 p-1),\left|\mu_{1}\right|=$ $(0,2 p)$ and $\left|\kappa_{0}\right|=(1,1)$. Here $\kappa_{0}$ is represented by $[d p]$ in the bar complex computing Tor, and $\Gamma\left(\kappa_{0}\right)=\mathbb{F}_{p}\left\{\gamma_{i}\left(\kappa_{0}\right) \mid i \geq 0\right\}$ denotes the divided power algebra on $\kappa_{0}$.

The inclusion $\operatorname{THH}\left(\mathbb{Z}_{p}\right) \rightarrow \operatorname{THH}\left(\mathbb{Z}_{p},\langle p\rangle\right)$ takes $\lambda_{1}$ to zero, so there is a differential

$$
d^{p}\left(\gamma_{p}\left(\kappa_{0}\right)\right)=\lambda_{1}
$$

up to a unit in $\mathbb{F}_{p}$, leaving the $E^{\infty}$-term

$$
E_{* *}^{\infty}=E(d \log p) \otimes P\left(\mu_{1}\right) \otimes P_{p}\left(\kappa_{0}\right)
$$

where $P_{p}\left(\kappa_{0}\right)=P\left(\kappa_{0}\right) /\left(\kappa_{0}^{p}\right)$ is the truncated polynomial algebra on $\kappa_{0}$ of height $p$. There is a multiplicative extension

$$
\kappa_{0}^{p}=\mu_{1}
$$


in total degree $2 p$, so that

$$
\pi_{*}\left(\mathrm{THH}\left(\mathbb{Z}_{p},\langle p\rangle\right) ; \mathbb{Z} / p\right) \cong E(d \log p) \otimes P\left(\kappa_{0}\right)
$$

as an algebra, with $|d \log p|=1$ and $\left|\kappa_{0}\right|=2$. Hence there is an abstract isomorphism

$$
\pi_{*}\left(\operatorname{THH}\left(\mathbb{Z}_{p},\langle p\rangle\right) ; \mathbb{Z} / p\right) \cong \pi_{*}\left(\operatorname{THH}\left(\mathbb{Z}_{p} \mid \mathbb{Q}_{p}\right) ; \mathbb{Z} / p\right)
$$

where $\operatorname{THH}\left(\mathbb{Z}_{p} \mid \mathbb{Q}_{p}\right)$ is as defined by Hesselholt-Madsen [29, Section 1.5]. We conjecture that this isomorphism is realized by an equivalence

$$
\operatorname{THH}\left(\mathbb{Z}_{p},\langle p\rangle\right) \simeq \operatorname{THH}\left(\mathbb{Z}_{p} \mid \mathbb{Q}_{p}\right)
$$

of cyclic commutative $\operatorname{THH}\left(\mathbb{Z}_{p}\right)$-algebras.

Example 8.14 More generally, Hesselholt and Madsen [29] consider local fields $K$ (complete discrete valuation fields of characteristic zero with perfect residue field $k$ of characteristic $p \neq 2$ ) with valuation ring $A \subset K$ and uniformizer $\pi$. Let $\alpha: M=$ $\langle\pi\rangle \rightarrow(A, \cdot)$ be the inclusion, and let $W=W(k)$ be the Witt ring. As explained in Serre [75, Section I.6, Proposition 18], the minimal polynomial $\phi(x)$ of $\pi \in A$ over $W$ has the form $\phi(x)=x^{e}-p \theta(x)$, where $e$ is the ramification index of $K$ and $\theta(x)$ is of degree $<e$ with $\theta(0)$ a unit. The Kähler differentials $\Omega_{A / W}^{1} \cong A /\left(\phi^{\prime}(\pi)\right)\{d \pi\}$ are generated by $d \pi$ with annihilator ideal the different $\left(\phi^{\prime}(\pi)\right) \subset A$, while the log Kähler differentials $\Omega_{(A, M) / W}^{1} \cong A /\left(\pi \phi^{\prime}(\pi)\right)\{d \log \pi\}$ are generated by $d \log \pi$ with annihilator ideal $\left(\pi \phi^{\prime}(\pi)\right) \subset(p) \subset A$. As explained in [29, Section 2.2] there is a natural short exact sequence

$$
0 \rightarrow \Omega_{A / W}^{1} \stackrel{\bar{\psi}}{\rightarrow} \Omega_{(A, M) / W}^{1} \stackrel{\text { res }}{\rightarrow} k \rightarrow 0
$$

where $\bar{\psi}(d \pi)=\pi d \log \pi$ and $\operatorname{res}(d \log \pi)=1$. In [29, 1.5.5], Hesselholt and Madsen define a useful ad hoc model $\mathrm{THH}(A \mid K)$ for the log topological Hochschild homology of $(A, M)$, such that there is a homotopy cofiber sequence

$$
\mathrm{THH}(k) \stackrel{i_{*}}{\rightarrow} \mathrm{THH}(A) \stackrel{j^{*}}{\rightarrow} \mathrm{THH}(A \mid K)
$$

where $i_{*}$ is the transfer map associated to the surjection $i: A \rightarrow A /(\pi)=k$, and $j^{*}$ is the natural map associated to the inclusion $j: A \rightarrow A\left[\pi^{-1}\right]=K$. In $[29,2.4 .1]$ they prove that

$$
\pi_{*}(\mathrm{THH}(A \mid K) ; \mathbb{Z} / p) \cong A / p \otimes E(d \log \pi) \otimes P\left(\kappa_{0}\right)
$$

where $A / p \otimes E(d \log \pi) \cong \Omega_{(A, M)}^{*} / p$ is the $\bmod p$ reduction of the $\log$ de Rham complex of $(A, M)$, and $\left|\kappa_{0}\right|=2$. 
Conjecturally, the following isomorphism is induced by an equivalence

$$
\operatorname{THH}(A,\langle\pi\rangle) \simeq \operatorname{THH}(A \mid K)
$$

of cyclic commutative $\operatorname{THH}(A)$-algebras.

Proposition 8.15 Let $A,\langle\pi\rangle$ and $K$ be as above. There is an isomorphism

$$
\pi_{*}(\operatorname{THH}(A,\langle\pi\rangle) ; \mathbb{Z} / p) \cong \pi_{*}(\operatorname{THH}(A \mid K) ; \mathbb{Z} / p)
$$

of $\pi_{*}(\mathrm{THH}(A) ; \mathbb{Z} / p)$-algebras.

Proof We will only prove this in the wildly ramified case, when $p \mid e$. One can use descent arguments, like in Hesselholt-Madsen [29, Section 2.4], to deal with the tamely ramified $(p \nmid e)$ and unramified $(e=1)$ cases.

We have a pushout square

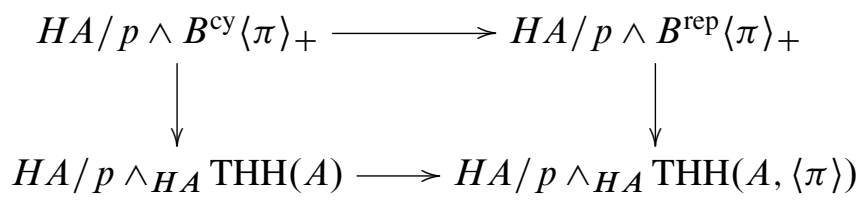

of commutative $H A / p$-algebras, and an associated Künneth spectral sequence

$$
\begin{aligned}
E_{* *}^{2} & =\operatorname{Tor}_{* *}^{H_{*}\left(B^{\mathrm{cy}}\langle\pi\rangle ; A / p\right)}\left(\pi_{*}(\operatorname{THH}(A) ; \mathbb{Z} / p), H_{*}\left(B^{\mathrm{rep}}\langle\pi\rangle ; A / p\right)\right) \\
& \Longrightarrow \pi_{*}(\operatorname{THH}(A,\langle\pi\rangle) ; \mathbb{Z} / p) .
\end{aligned}
$$

In the wildly ramified case,

$$
\pi_{*}(\mathrm{THH}(A) ; \mathbb{Z} / p) \cong A / p \otimes E\left(\alpha_{1}\right) \otimes P\left(\alpha_{2}\right)
$$

by Lindenstrauss-Madsen [41, Theorem 4.4(ii)], with $\left|\alpha_{1}\right|=1$ and $\left|\alpha_{2}\right|=2$, and $j^{*}$ takes $\alpha_{2}$ to a unit times $\kappa_{0}$. Hence the $E^{2}$-term is isomorphic to

$$
E_{* *}^{2} \cong \operatorname{Tor}_{* *}^{E(d \pi)}\left(A / p \otimes E\left(\alpha_{1}\right) \otimes P\left(\alpha_{2}\right), E(d \log \pi)\right)
$$

where $d \pi \mapsto \alpha_{1}$ and $d \pi \mapsto \pi d \log \pi$ in the respective factors. Hence the $E^{2}$-term is concentrated on the vertical axis, the Künneth spectral sequence collapses, and we get the isomorphism

$$
\begin{aligned}
\pi_{*}(\operatorname{THH}(A,\langle\pi\rangle) ; \mathbb{Z} / p) & \cong\left(A / p \otimes E\left(\alpha_{1}\right) \otimes P\left(\alpha_{2}\right)\right) \otimes_{E(d \pi)} E(d \log \pi) \\
& \cong A / p \otimes E(d \log \pi) \otimes P\left(\alpha_{2}\right) .
\end{aligned}
$$

The abstract isomorphism with $\pi_{*}(\operatorname{THH}(A \mid K) ; \mathbb{Z} / p)$ takes $\alpha_{2}$ to a unit times $\kappa_{0}$. 
Definition 8.16 Let $N$ be a commutative based $\mathcal{I}$-space monoid over $S^{0}$, as in Definition 6.19. The based cyclic bar construction on $N$ is $B_{\wedge}^{\mathrm{cy}} N=S_{\bullet}^{1} \otimes N$, where the tensor product is formed in $\left(\mathcal{S}_{0}^{\mathcal{I}}, \square, S^{0}\right)$. The suspension spectrum $\Sigma^{\infty} N$ is a commutative symmetric ring spectrum, and

$$
\operatorname{THH}\left(\Sigma^{\bullet} N\right)=S_{\bullet}^{1} \otimes \Sigma^{\bullet} N \cong \Sigma^{\bullet}\left(S_{\bullet}^{1} \otimes N\right)=\Sigma^{\bullet} B_{\wedge}^{\mathrm{cy}} N .
$$

Now suppose that $N=\operatorname{cone}(L) \cup_{L} N^{\prime}$ is a commutative conically based $\mathcal{I}$-space monoid. Based on discussions of symmetric conically based $\mathcal{I}$-space monoid derivations, like Definition 5.14 and Lemma 12.4, we are led to declare the based replete bar construction of $N$ to be

$$
B_{\wedge}^{\text {rep }} N=N \boxminus B\left(\Gamma N^{\prime}\right)_{+} \cdot
$$

Discussions similar to Lemma 5.19 and Proposition 12.7 specify the repletion map

$$
\psi: B_{\wedge}^{\mathrm{cy}} N \rightarrow B_{\wedge}^{\text {rep }} N
$$

up to homotopy, but it is best described as the suspension of the based shear map sh: $N \bullet N \rightarrow N \oslash\left(\Gamma N^{\prime}\right)_{+}$given in Definition 13.14. Here the suspension is formed in the category of commutative $\mathcal{I}$-space monoids under and over $N$.

Definition 8.17 Let $(A, N, \alpha)$ be a conically based pre-log symmetric ring spectrum. The based log topological Hochschild homology $\operatorname{THH}_{0}(A, M)$ of $(A, M)$ is defined by the pushout square

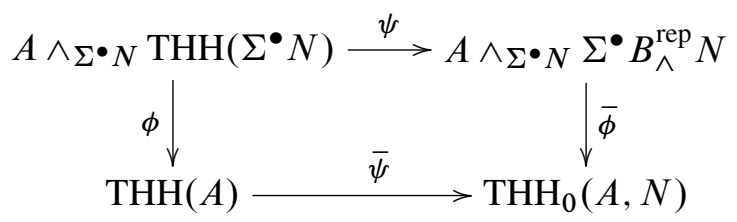

of commutative $A$-algebras. Here $A \wedge_{\Sigma^{\bullet} N} \Sigma^{\bullet} B_{\wedge}^{\text {rep }} N \simeq A \wedge B\left(\Gamma N^{\prime}\right)_{+} \simeq A \wedge B N_{+}^{\prime}$.

\section{Operadic logarithmic structures}

Definition 9.1 Let $A$ be a commutative $S$-algebra and let $o: \mathcal{O} \rightarrow \mathcal{L}$ be an operad augmented over the linear isometries operad, so that each $\mathcal{L}$-space $M$ has an underlying $\mathcal{O}$-space $o^{\#} M$. By an $\mathcal{O}$ pre-log structure on $A$ we mean a pair $(M, \alpha)$, where $M$ is an $\mathcal{L}$-space and $\alpha: o^{\#} M \rightarrow o^{\#} \Omega_{\otimes}^{\infty} A$ is a map of the underlying $\mathcal{O}$-spaces. A map

$$
\left(f, f^{b}\right):(A, M) \rightarrow(B, N)
$$


of $\mathcal{O}$ pre-log $S$-algebras is a map $f: A \rightarrow B$ of commutative $S$-algebras, and a map $f^{b}: M \rightarrow N$ of $\mathcal{L}$-spaces, such that the square

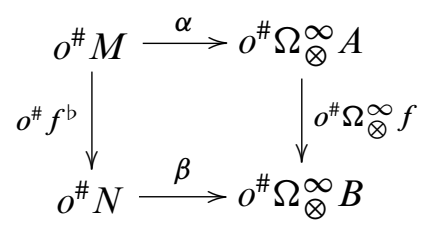

commutes in $\mathcal{O}[\mathcal{U}]$. To make homotopy-theoretic sense of this structure, we will need to cofibrantly replace $o^{\#} M$ (and $o^{\#} N$ ) in the category of $\mathcal{O}$-spaces. The category of $\mathcal{O}$ pre-log $S$-algebras is the comma category

$$
\mathcal{O P r e} \log (S)=\left(o^{\#}, o^{\#} \Omega_{\otimes}^{\infty}\right)
$$

where $o^{\#}: \mathcal{L}[\mathcal{U}] \rightarrow \mathcal{O}[\mathcal{U}]$ and $o^{\#} \Omega_{\otimes}^{\infty}: \mathcal{C}_{S} \rightarrow \mathcal{O}[\mathcal{U}]$; see Mac Lane [44, Section II.6].

When $\mathcal{O}$ is an $E_{n}$ operad, like the little $n$-cubes operad $\mathcal{C}_{n}$, we say that $(M, \alpha)$ is an $E_{n}$ pre-log structure on $A$. To make $\mathcal{C}_{n}$ augmented over $\mathcal{L}$, we will implicitly replace it by the product operad $\mathcal{C}_{n} \times \mathcal{L}$. Similarly, the category of $\mathcal{O}$ based pre-log $S$-algebras is defined to be $\left(o^{\#}, o^{\#} \Omega_{\otimes}^{\infty}\right)$, where now $o^{\#}: \mathcal{L}_{0}[\mathcal{T}] \rightarrow \mathcal{O}_{0}[\mathcal{T}]$ and $o^{\#} \Omega_{\otimes}^{\infty}: \mathcal{C}_{S} \rightarrow \mathcal{O}_{0}[\mathcal{T}]$.

Remark 9.2 In view of the fact that $\mathcal{C}_{n-1}$-algebras in associative $S$-algebras are $E_{n}$ ring spectra (see Brun-Fiedorowicz-Vogt [17, Theorem C]), we might model $E_{n}-$ algebras in $\mathcal{I}$-spaces by $\mathcal{C}_{n-1}$-algebras in associative $\mathcal{I}$-monoids, to get a definition of an $E_{n}$ pre-log structure on a commutative symmetric ring spectrum $A$. For $n=2$, this would consist of a commutative $\mathcal{I}$-space monoid $M$ and a map $\alpha: M \rightarrow \Omega_{\otimes}^{\bullet} A$ of $\mathcal{C}_{1}$-algebras in associative $\mathcal{I}$-space monoids.

Remark 9.3 When discussing topological André-Quillen homology for $A$ and $S[M]$, we will need $A$ and $M$ to be commutative or $E_{\infty}$ objects, and in order to form the log topological André-Quillen homology $\operatorname{TAQ}(A, M)$ for $(A, M)$ we will need that $\alpha$ is an $E_{\infty}$ map. On the other hand, when discussing topological Hochschild homology of $A$ and $S[M]$, we only need $A$ and $M$ to be associative or $A_{\infty}$ objects. However, to form the $\log$ topological Hochschild homology of $(A, M)$ we will make use of the repletion $B^{\text {rep }} M$ of the cyclic bar construction $B^{\text {cy }} M$ as a space over $M$. For an augmentation $\epsilon: B^{\text {cy }} M \rightarrow M$ to exist, extending the identity on the zero-simplices $M \subset B^{c y} M$, it is necessary and sufficient that $M$ is a cyclic $A_{\infty}$ space as defined eg by Vigleik Angeltveit [1, 4.1, 4.4] and Getzler-Kapranov [25]. This means that $M$ is homotopy-commutative in a somewhat strong sense. For example, $\epsilon$ must take each 1-simplex $(a, b)$ to a homotopy $\gamma_{a, b}$ from $a b$ to $b a$, and it must take each 
2-simplex $(a, b, c)$

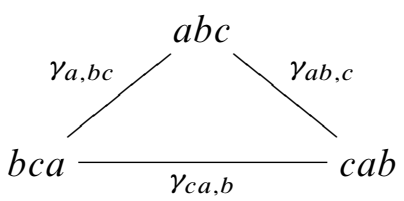

to a second order homotopy ( $=2$-cell) connecting the composite homotopy $\gamma_{a b, c} * \gamma_{c a, b}$ from $a b c$ via $c a b$ to $b c a$ to the direct homotopy $\gamma_{a, b c}$ from $a b c$ to $b c a$.
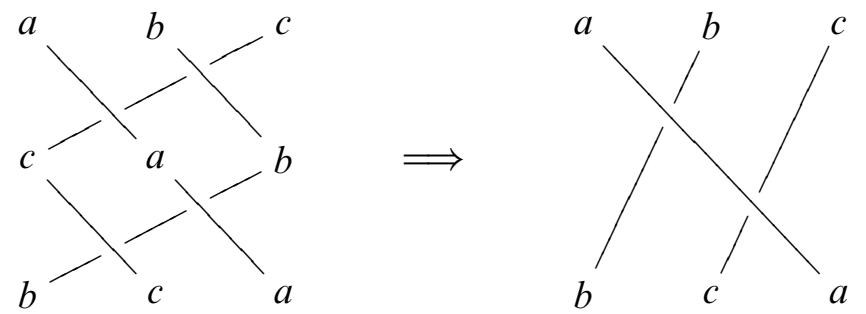

A general $E_{2}$ space $M$ will admit the homotopies $\gamma_{a, b}$, but might not admit the second order homotopy, since the full twist $\gamma_{b, c} * \gamma_{c, b}$ is often not homotopic to the identity. An $E_{3}$ space $M$ will admit the second homotopy, but also satisfies coherence conditions for noncyclic permutations that may not be required in a cyclic $A_{\infty}$ structure. To extend a retraction to $M$ from the 2-skeleton to the 3-skeleton of $B^{\text {cy }} M$ part of an $E_{4}$ structure will be needed, and so on. It would be interesting to know in operadic terms what it means for $B^{\mathrm{cy}} M$ to admit a retraction to $M$, but for our purposes it seems reasonable just to assume that $M$ is $E_{\infty}$, so that we can rectify it to a commutative $\mathcal{I}$-space monoid, for which the retraction $\epsilon: B^{\text {cy }} M \rightarrow M$ always exists.

In the case of grouplike $M$, Thomas Kragh has pointed out that if $M=\Omega X$, with $X$ an $H$-group, we have $B^{\text {cy }} M \simeq \Lambda X$ and $\Omega X \rightarrow \Lambda X$ admits a retraction, since the homotopy fiber sequence $\Omega X \rightarrow \Lambda X \rightarrow X$ admits a section and $\Lambda X$ is an $H$-group. Hence for grouplike $M$ it suffices that $B M$ is an $H$-group. For example, this applies to all grouplike $E_{2}$-spaces.

Assuming that $M$ is cyclic $A_{\infty}$, or $E_{\infty}$, we will need that $A$ and $\alpha$ are associative in order to define $\operatorname{THH}(A, M)$, but more commutativity in $A$ and $\alpha$ will give more multiplicative structure to $\operatorname{THH}(A, M)$. If $\alpha$ is a map of $E_{n}$ spaces, or more precisely, a map of $\mathcal{C}_{n-1}$-algebras in associative monoids, then $\operatorname{THH}(A, M)$ will be a $\mathcal{C}_{n-1}$ algebra in spectra, ie, an $E_{n-1}$ ring spectrum. As the following lemmas show, this seems to be a relevant setting for topological log geometry over the sphere spectrum.

Definition 9.4 Let $A$ be an associative $S$-algebra, and let $x \in \Omega_{\otimes}^{\infty} A$ be a chosen point. Let $M=\bigsqcup_{j \geq 0} \mathcal{L}(j)$ be the free non- $\Sigma \mathcal{L}$-space on a single point $\{1\}$, and 
extend the map $1 \mapsto x$ freely to a map $\bar{x}: M \rightarrow \Omega_{\otimes}^{\infty} A$ of non- $\Sigma \mathcal{L}$-spaces $\left(=A_{\infty}\right.$ spaces), taking the contractible space $\mathcal{L}(j)$ to the path component of $x^{j}$, for each $j \geq 0$. Let $\langle x\rangle=\left\{1, x, x^{2}, \ldots\right\}$ be the free associative (and commutative) monoid generated by $x$. The collapse map $M \rightarrow\langle x\rangle$ is an equivalence of $A_{\infty}$ spaces. We call $(M, \bar{x})$ the free $A_{\infty}$ pre-log structure on $A$ generated by $x$, and usually denote it by $(\langle x\rangle, \bar{x})$ or $\langle x\rangle$.

If $A$ is an $E_{n}$ ring spectrum, or more precisely a $\mathcal{C}_{n-1}$-algebra in associative $S$ algebras, then $\Omega_{\otimes}^{\infty} A$ is a $\mathcal{C}_{n-1}$-algebra in non- $\Sigma \mathcal{L}$-spaces, hence is equivalent to a $\mathcal{C}_{n}$-space. Suppressing this equivalence, each point $x \in \Omega_{\otimes}^{\infty} A$ specifies a $\mathcal{C}_{n}$-map $\bar{x}: C_{n} S^{0} \rightarrow \Omega_{\otimes}^{\infty} A$, where $C_{n} S^{0}$ is the free $\mathcal{C}_{n}$-space on one generator. We call $\left(C_{n} S^{0}, \bar{x}\right)$ the free $E_{n}$ pre-log structure on $A$ generated by $x$.

Lemma 9.5 Let $p$ be a prime and write $H_{*}(X)$ for $H_{*}\left(X ; \mathbb{F}_{p}\right)$. Let $A$ be an $E_{2}$ ring spectrum and $x \in \Omega_{\otimes}^{\infty} A$ a point. If $p$ is odd, assume that $[x] \in H_{0}\left(\Omega_{\otimes}^{\infty} A\right)$ has trivial Browder operation $\lambda_{1}([x],[x])=0$ in $H_{1}\left(\Omega_{\otimes}^{\infty} A\right)$. If $p=2$, assume that Cohen's "top" operation $\xi_{1}([x])=0$ in $H_{1}\left(\Omega_{\otimes}^{\infty} A\right)$. Both hypotheses are trivially satisfied if $H_{1}\left(\Omega_{\otimes}^{\infty} A\right)=0$. Then the algebra homomorphism

$$
\bar{x}_{*}: H_{*}\left(C_{2} S^{0}\right) \rightarrow H_{*}\left(\Omega_{\otimes}^{\infty} A\right)
$$

induced by the free $E_{2}$ pre-log structure $\bar{x}: C_{2} S^{0} \rightarrow \Omega_{\otimes}^{\infty} A$ is zero in positive degrees, hence factors through the augmentation

$$
H_{*}\left(C_{2} S^{0}\right) \rightarrow H_{*}(\langle x\rangle)=P(e) .
$$

In other words, there is no mod $p$ homological obstruction to there being an $E_{2}$ pre$\log$ structure $(M, \alpha)$ on $A$, with $M \simeq\langle x\rangle$, so that the composite $C_{2} S^{0} \rightarrow\langle x\rangle \simeq$ $M \stackrel{\alpha}{\longrightarrow} \Omega_{\otimes}^{\infty} A$ is homotopic to $\bar{x}$.

Proof By Fred Cohen's calculation [18, III.A.1],

$$
H_{*}\left(C_{2} S^{0}\right)=P(e) \otimes E\left(h_{i} \mid i \geq 0\right) \otimes P\left(g_{i} \mid i \geq 1\right)
$$

for $p$ odd, where $P$ and $E$ indicate the polynomial and exterior algebras on the listed generators, respectively. Here $e=[1], h_{0}=\lambda_{1}(e, e), h_{i}=\xi_{1}\left(h_{i-1}\right)$ and $g_{i}=\beta h_{i}$ for all $i \geq 1$, where the Browder operation $\lambda_{1}$ (which is 0 for all $E_{3}$ spaces) and the top operation $\xi_{1}$ are defined in [18, Section III.1], while $\beta$ is the Bockstein operation. See also Yamaguchi [82, page 522]. The $E_{2}$-map $\bar{x}$ takes $e$ to $[x]$ and $h_{0}$ to $\lambda_{1}([x],[x])$, which is 0 by assumption. By naturality of the operations it follows that also $h_{i}$ and $g_{i}$ map to 0 , for all $i \geq 1$, so $\bar{x}_{*}$ factors through the augmentation to $P(e)=H_{*}(\langle x\rangle)$. The proof for $p=2$ is very similar. 
Lemma 9.6 There is no $E_{3}$ pre-log structure $(M, \alpha)$ on $k u$ with $M \simeq\langle p\rangle$, such that the (homotopy) generator maps to a point in the $p$-th component of $\Omega_{\otimes}^{\infty} k u \simeq$ $(B U \times \mathbb{Z})_{\otimes}$. The same conclusion applies for the $p$-completion $k u_{p}$ of $k u$. Hence there is no such $E_{3}$ pre-log structure on any other commutative $S$-algebra $A$ with a commutative $S$-algebra map to $k u_{p}$.

Proof For $\mathcal{C}_{3}$-spaces $M$ there is a top operation $\xi_{2}: H_{0}(M) \rightarrow H_{2 p-2}(M)$ that agrees with $Q^{1}$ for $E_{4}$ spaces. The generator $e=[1] \in H_{0}(M)$ maps to $[p] \in$ $H_{0}(B U \times \mathbb{Z})$, so if $\alpha$ is a $\mathcal{C}_{3}$-map the class $\xi_{2}(e)$ in $H_{2 p-2}(M)=0$ maps to $\widetilde{Q}^{1}[p]$ in $H_{2 p-2}(B U \times \mathbb{Z})$, where $\widetilde{Q}^{r}$ denotes the multiplicative Dyer-Lashof operation. Now

$$
\widetilde{Q}^{r}[p] \equiv-Q^{r}[1] *\left[p^{p}-p\right]
$$

modulo $*$-decomposables by Cohen-Lada-May [18, II.2.8], and

$$
Q^{r}[1] \equiv-(-1)^{r} b_{r(p-1)} *[p]
$$

modulo $*$-decomposables by [18, II.7.1]. Here $H_{*}(B U)=P\left(b_{i} \mid i \geq 1\right)$ with $\left|b_{i}\right|=2 i$, where $b_{i}$ is the image of a generator of $H_{2 i}(B U(1))$ under the inclusion $B U(1) \subset B U \times\{0\} \subset B U \times \mathbb{Z}$. Hence $\widetilde{Q}^{1}[p] \equiv-b_{p-1} *\left[p^{p}\right] \neq 0$ in $H_{2 p-2}(B U \times \mathbb{Z})$. In particular, it cannot be the image under $\alpha_{*}$ of $\xi_{2}(e)=0$.

These $\bmod p$ homological calculations hardly distinguish between $k u$ and $k u_{p}$. The last conclusion follows by naturality, since an $E_{3}$ pre-log structure $\alpha: M \rightarrow \Omega_{\otimes}^{\infty} A$ composed with an $E_{\infty}$ map $\Omega_{\otimes}^{\infty} A \rightarrow \Omega_{\otimes}^{\infty} k u_{p}$ would produce an $E_{3}$ pre-log structure on $k u_{p}$.

Lemma 9.7 Let $A$ be a commutative $S$-algebra such that the unit map $S \rightarrow A$ takes the Hopf map $\eta \in \pi_{1}(S)$ to zero in $\pi_{1}(A)[1 / p]$. For simplicity assume that $\mathbb{Z} \cong \pi_{0}(S) \rightarrow \pi_{0}(A)$ is injective, and write $N \times\langle p\rangle \subset \Omega_{\otimes}^{\infty} A$ for the sub-L-space consisting of the path components corresponding to $\langle p\rangle \subset \pi_{0}(A)$. Consider the group completion

$$
\Gamma(\bar{p}): \Gamma\left(C_{2} S^{0}\right) \rightarrow \Gamma(N \times\langle p\rangle)
$$

of the $\mathcal{C}_{2}-$ map $\bar{p}: C_{2} S^{0} \rightarrow N \times\langle p\rangle$ freely generated by $1 \mapsto p$. Here $\Gamma\left(C_{2} S^{0}\right) \simeq$ $\Omega^{2} S^{2}$ and $\Gamma(N \times\langle p\rangle) \simeq N[1 / p] \times\left\langle p, p^{-1}\right\rangle$. Restricted to the $0-$ th component in the source,

$$
\Gamma_{0}(\bar{p}): \Omega_{0}^{2} S^{2} \rightarrow N[1 / p]
$$

is null-homotopic as a $\mathcal{C}_{2}-$ map.

Proof The additive group completion equivalence $\Gamma\left(C_{2} S^{0}\right) \simeq \Omega^{2} S^{2}$ is due to Graeme Segal [74, Theorem 1] (see also Cohen-Lada-May [18, III.3.3]). The multiplicative 
group completion equivalence $\Gamma(N \times\langle p\rangle) \simeq N[1 / p] \times\left\langle p, p^{-1}\right\rangle$ is due to Peter May [46, VII.5.3], generalizing a result of Jørgen Tornehave. The Hopf fiber sequence $S^{1} \rightarrow S^{3} \stackrel{\eta}{\longrightarrow} S^{2}$ loops to a fiber sequence $\Omega S^{3} \rightarrow \Omega S^{2} \rightarrow S^{1}$ with a section, so there are equivalences

$$
\Omega^{2} S^{3} \times \mathbb{Z} \stackrel{\simeq}{\longleftarrow} \Omega^{2} S^{3} \times \Omega S^{1} \stackrel{\simeq}{\rightarrow} \Omega^{2} S^{2}
$$

of $\Omega$-spaces. The inclusion $\Omega^{2} \eta: \Omega^{2} S^{3} \rightarrow \Omega_{0}^{2} S^{2}$ of the zero-th component is an $\Omega^{2}$-equivalence, and the composite

$$
C_{2} S^{1} \stackrel{\simeq}{\rightarrow} \Omega^{2} S^{3} \stackrel{\simeq}{\rightarrow} \Omega_{0}^{2} S^{2} \rightarrow \Gamma_{1}(N \times\langle p\rangle) \simeq N[1 / p]
$$

is the free $\mathcal{C}_{2}$-map generated by its restriction $S^{1} \rightarrow N[1 / p]$, representing the image of $\eta$ in $\pi_{1}(N[1 / p]) \cong \pi_{1}(A)[1 / p]$. By assumption the map from $S^{1}$ is null-homotopic as a based map, hence the free $\mathcal{C}_{2}$-map it generates is null-homotopic as a $\mathcal{C}_{2}$-map.

We view these lemmas as motivation for the following hypothesis.

Hypothesis 9.8 Let $A$ be an $E_{2}$ ring spectrum with $\pi_{1}(A)=0$, and let $x \in \Omega_{\otimes}^{\infty} A$. Then the free $A_{\infty}$ pre-log structure $(\langle x\rangle, \bar{x})$ on $A$ generated by $x$ lifts to an $E_{2}$ pre-log structure $(M, \alpha)$ on $A$, with $M \simeq\langle x\rangle$ and $\alpha$ homotopic to $\bar{x}$.

Definition 9.9 Let $A$ be a commutative $S$-algebra, let $Y=S^{d}$ be a sphere, and let $y: S^{d} \rightarrow \Omega_{\otimes}^{\infty} A$ be a based map representing a homotopy class in $\pi_{d}(A)$ with Hurewicz image $[y] \in H_{d}\left(\Omega_{\otimes}^{\infty} A\right)$. There are canonical maps

$$
\begin{aligned}
C_{1,0} S^{d} & =\bigvee_{j \geq 0} \mathcal{C}_{1}(j)_{+} \wedge_{\Sigma_{j}} S^{d j} \simeq \bigvee_{j \geq 0} S^{d j} \\
& \rightarrow C_{2,0} S^{d}=\bigvee_{j \geq 0} \mathcal{C}_{2}(j)_{+} \wedge_{\Sigma_{j}} S^{d j} \simeq \bigvee_{j \geq 0} S_{h B_{j}}^{d j} \\
& \rightarrow L_{0} S^{d}=\bigvee_{j \geq 0} \mathcal{L}(j)_{+} \wedge_{\Sigma_{j}} S^{d j} \simeq \bigvee_{j \geq 0} S_{h \Sigma_{j}}^{d j},
\end{aligned}
$$

where $B_{j}$ is the $j$-th braid group. Let $\sigma_{j}$ be the $\mathbb{R}^{j}$-bundle over $B \Sigma_{j}$ associated to the usual inclusion $\Sigma_{j} \rightarrow O(j)$, and let $\beta_{j}$ be the $\mathbb{R}^{j}$-bundle over $B B_{j}$ associated to the composite homomorphism $B_{j} \rightarrow \Sigma_{j} \rightarrow O(j)$, so that $\beta_{j}$ is the pullback of $\sigma_{j}$ along the usual map $B B_{j} \rightarrow B \Sigma_{j}$. Then $S_{h \Sigma_{j}}^{d j}=E \Sigma_{j+} \wedge \Sigma_{j} S^{d j} \cong T h\left(d \sigma_{j}\right)$ is the Thom complex of $d$ times $\sigma_{j}$, and $S_{h B_{j}}^{d j}=E B_{j+} \wedge_{B_{j}} S^{d j} \cong T h\left(d \beta_{j}\right)$ is the Thom complex of $d$ times $\beta_{j}$. From here on we assume that $d$ is even. 
Proposition 9.10 When $Y=S^{d}$ is an even sphere each vector bundle $d \beta_{j}$ over $B B_{j}$ is trivial, so $S_{h B_{j}}^{d j} \cong \Sigma^{d j}\left(B B_{j+}\right)$. Hence each inclusion $S^{d j} \rightarrow S_{h B_{j}}^{d j}$ admits a retraction $r_{j}: S_{h B_{j}}^{d j} \rightarrow S^{d j}$, and these combine to a retraction of based spaces

$$
r: C_{2,0} S^{d} \rightarrow C_{1,0} S^{d} .
$$

Proof For $d=2$ there is a trivialization of $2 \beta_{j}$, given in Cohen-Mahowald-Milgram $[19$, Theorem 1] by an explicit map

$$
v: \mathcal{C}_{2}(j) \times \Sigma_{j}\left(\mathbb{R}^{2}\right)^{j} \rightarrow\left(\mathbb{R}^{2}\right)^{j} .
$$

To each $j$-tuple $c=\left(c_{1}, \ldots, c_{j}\right)$ of little squares ( $=2$-cubes) in $I^{2}$ we can associate a $j$-tuple $z=\left(z_{1}, \ldots, z_{j}\right)$ of distinct points in $I^{2} \subset \mathbb{R}^{2}$, given by the barycenters of the squares. Identifying $\mathbb{R}^{2}$ with $\mathbb{C}$, we let

$$
v(z, \xi)=\left(\sum_{i} \xi_{i}, \sum_{i} z_{i} \xi_{i}, \ldots, \sum_{i} z_{i}^{j-1} \xi_{i}\right)
$$

for $\xi=\left(\xi_{1}, \ldots, \xi_{j}\right)$ in $\left(\mathbb{R}^{2}\right)^{j} \cong \mathbb{C}^{j}$. Simultaneously reordering the $z_{i}$ and $\xi_{i}$ by a permutation in $\Sigma_{j}$ does not change these sums, so $v$ is well-defined. For a fixed $z=\left(z_{1}, \ldots, z_{j}\right)$ the linear map $\xi \mapsto v(z, \xi)$ is given by a Vandermonde matrix, which is nonsingular because the $z_{i}$ are all distinct. Taking the Whitney sum of $(d / 2)$ copies of this trivialization we get a trivialization of $d \beta_{j}$.

Remark 9.11 We would like to know if there is a based $E_{2}$ structure on $C_{1,0} S^{d} \simeq$ $\bigvee_{j \geq 0} S^{d j}$ such that the retraction $r: C_{2,0} S^{d} \rightarrow C_{1,0} S^{d}$ is an $E_{2}$ map. The composite

$$
C_{2,0}\left(C_{1,0} S^{d}\right) \stackrel{i}{\rightarrow} C_{2,0}\left(C_{2,0} S^{d}\right) \stackrel{\mu}{\rightarrow} C_{2,0} S^{d} \stackrel{r}{\rightarrow} C_{1,0} S^{d}
$$

where $i$ is induced by the inclusion, and $\mu$ expresses composition in the operad $\mathcal{C}_{2}$, decomposes as a wedge sum of maps

$$
\mathcal{C}_{2}(j)_{+} \wedge_{G}\left(S^{d i_{1}} \wedge \cdots \wedge S^{d i_{j}}\right) \rightarrow S^{d|i|},
$$

where $G \subset \Sigma_{j}$ is the stabilizer of $\left(i_{1}, \ldots, i_{j}\right)$ and $|i|=i_{1}+\cdots+i_{j}$. This can be modeled by cabling, taking $\mathcal{C}_{2}(j)$ to $\mathcal{C}_{2}(|i|)$, and using the retraction $\mathcal{C}_{2}(|i|)_{+} \wedge_{G} S^{d|i|} \rightarrow S^{d|i|}$. For a more rigid model, we might replace $\mathcal{C}_{2}(j)$ by the homotopy-equivalent space $B P_{j}$, where $P_{j} \subset B_{j}$ is the pure braid group on $j$ strings, and use the cabling map $B P_{j} \rightarrow B P_{|i|}$. We do not know if there is a map

$$
B P_{j+} \wedge_{G}\left(S^{d i_{1}} \wedge \cdots \wedge S^{d i_{j}}\right) \rightarrow S^{d|i|}
$$

that generates a based $E_{2}$ structure on $\bigvee_{j \geq 0} S^{d j}$. 
Lemma 9.12 If $\lambda_{1}([y],[y])=0$ in $H_{2 d+1}\left(\Omega_{\otimes}^{\infty} A\right)$, as it is for any $E_{3}$ ring spectrum $A$, then the algebra homomorphism

$$
\left(\bar{y}_{0}\right)_{*}: \tilde{H}_{*}\left(C_{2,0} S^{d}\right) \rightarrow H_{*}\left(\Omega_{\otimes}^{\infty} A\right)
$$

induced by the free $E_{2}$ based pre-log structure factors through the retraction

$$
r_{*}: \widetilde{H}_{*}\left(C_{2,0} S^{d}\right) \rightarrow \widetilde{H}_{*}\left(C_{1,0} S^{d}\right) \cong P(e),
$$

where $e \in \widetilde{H}_{d}\left(S^{d}\right) \subset \widetilde{H}_{*}\left(C_{1,0} S^{d}\right)$ is the fundamental class.

Proof We have isomorphisms

$$
\begin{aligned}
\tilde{H}_{*}\left(C_{2,0} S^{d}\right) & \cong \bigoplus_{j \geq 0} H_{*}\left(B B_{j} ; \mathbb{F}_{p}\left\{e^{j}\right\}\right) \\
& \cong P(e) \otimes E\left(h_{i} \mid i \geq 0\right) \otimes P\left(g_{i} \mid i \geq 1\right) .
\end{aligned}
$$

Since $d$ is even, $B_{j}$ acts trivially on $\mathbb{F}_{p}\left\{e^{j}\right\}$. We have $h_{0}=\lambda_{1}(e, e), h_{i}=\xi_{1}\left(h_{i-1}\right)$ and $g_{i}=\beta h_{i}$ for $i \geq 1$. Here $|e|=d,\left|h_{i}\right|=2 p^{i}(d+1)-1$ and $\left|g_{i}\right|=2 p^{i}(d+1)-2$. (These conventions specialize to those used in the proof of Lemma 9.5 when $d=0$.) The retraction $r_{*}$ takes $e$ to $e$ and maps each $h_{i}$ and $g_{i}$ to zero. The $\mathcal{C}_{2}$-map $\bar{y}_{0}$ takes $e$ to $[y]$, so if $\lambda_{1}([y],[y])=0$ then all $h_{i}$ and $g_{i}$ map to zero in $H_{*}\left(\Omega_{\otimes}^{\infty} A\right)$. Hence $\left(\bar{y}_{0}\right)_{*}$ factors through $r_{*}$, as claimed.

Lemma 9.13 There is no $E_{3}$ pre-log structure $(M, \alpha)$ on $k u$ with $M \simeq C_{1,0} S^{2}$, such that the generator $e$ of $H_{2}(M) \subset \widetilde{H}_{*}\left(C_{1,0} S^{2}\right) \cong P(e)$ maps to $[u]$ in $H_{2}(B U) \subset$ $H_{*}(B U \times \mathbb{Z})$, where $[u]$ is the Hurewicz image of the Bott class $u: S^{2} \rightarrow B U$.

Proof We write $H_{*}(B U)=P\left(b_{i} \mid i \geq 1\right)$, as in the proof of Lemma 9.6, so $[u]=b_{1}$. There is a natural operation $\xi_{2}: H_{2}(M) \rightarrow H_{4 p-2}(M)$ for $\mathcal{C}_{3}$-spaces, which agrees with $Q^{2}$ for $E_{4}$ spaces by Cohen-Lada-May [18, III.1.3]. The Bott class [u] is primitive, so

$$
\widetilde{Q}^{r}([u] *[1])=\left(\widetilde{Q}^{r}[u]\right) *[1]+\left(Q^{r}[u]\right) *[1]
$$

by [18, II.8.6]. Here $\widetilde{Q}^{r}([u] *[1])=0$ for $r>0$ by [18, II.7.2], since $B U(1) \times 1 \rightarrow$ $\Omega_{\otimes}^{\infty} k u$ is an $E_{\infty}$ map from a strictly commutative monoid. Furthermore,

$$
Q^{r}[u] \equiv(-1)^{r}(r-1) b_{r(p-1)+1}
$$

modulo $*$-decomposables by Kochman's calculations [37, Theorem 6], so $\widetilde{Q}^{2}[u] \equiv$ $-b_{2 p-1} \neq 0$ modulo $*$-decomposables. In particular, $\widetilde{Q}^{2}[u]$ cannot be the image under $\alpha_{*}$ of $\xi_{2}(e)=0$ in $H_{*}(M)$. 
Remark 9.14 The graded analogue of Lemma 9.7 is presently hypothetical. The stable Snaith splitting [76] of $C_{2} S^{d}$ induces an isomorphism

$$
\tilde{H}_{*}\left(C_{2,0} S^{d}\right) \cong H_{*}\left(C_{2} S^{d}\right) \cong P(e) \otimes E\left(h_{i} \mid i \geq 0\right) \otimes P\left(g_{i} \mid i \geq 1\right) .
$$

There is no obvious notion of group completion in the category of based $E_{\infty}$ spaces, but there may be a suitable category of graded $E_{\infty}$ spaces, or commutative monoids in graded $\mathcal{I}$-spaces, where this makes sense. See Sagave-Schlichtkrull [67].

To recover the summands $\widetilde{H}_{*}\left(S_{h B_{j}}^{d j}\right) \cong H_{*}\left(B B_{j} ; \mathbb{F}_{p}\left\{e^{j}\right\}\right)$ in $\widetilde{H}_{*}\left(C_{2,0} S^{d}\right)$ one introduces a weight function $w$, with $w(e)=1, w\left(h_{i}\right)=2 p^{i}$ and $w\left(g_{i}\right)=2 p^{i}$. The monomials of total weight $j$ then form a basis for $H_{*}\left(B B_{j} ; \mathbb{F}_{p}\left\{e^{j}\right\}\right)$. If we assume that this (nonconnective) graded group completion has the effect of inverting the fundamental class $e$, the weight zero component of the result has homology

$$
H_{*}\left(\Gamma_{0} C_{2,0} S^{d}\right) \cong E\left(\bar{h}_{i} \mid i \geq 0\right) \otimes P\left(\bar{g}_{i} \mid i \geq 1\right)
$$

where $\bar{h}_{i}=e^{-2 p^{i}} h_{i}$ and $\bar{g}_{i}=e^{-2 p^{i}} g_{i}$ all have weight zero. This algebra is isomorphic to

$$
\widetilde{H}_{*}\left(C_{2,0} S^{1}\right) \cong H_{*}\left(C_{2} S^{1}\right) \cong H_{*}\left(\Omega^{2} S^{3}\right) .
$$

We can reach the same result from a different point of view, involving the conically based spaces. With $N=C_{2,0} S^{d} \simeq \bigvee_{j \geq 0} S_{h B_{j}}^{d j}$ the base point complement $N^{\prime} \simeq \bigsqcup_{j \geq 0} B B_{j} \simeq C_{2} S^{0}$ has group completion $\Gamma N^{\prime} \simeq \Omega^{2} S^{2}$, and its zero-th path component is $\Gamma_{0} N^{\prime} \simeq \Omega_{0}^{2} S^{2} \simeq \Omega^{2} S^{3}$. Hence the obstruction, in the base point component after group completion, to improving a free $A_{\infty}$ based pre-log structure generated by a map $S^{d} \rightarrow \Omega_{\otimes}^{\infty} A$ into an $E_{2}$ based pre-log structure, lies in the $E_{2}$ map $\Omega^{2} S^{3} \rightarrow \Omega_{\otimes}^{\infty} A$ generated by $\eta$.

Hypothesis 9.15 Let $A$ be an $E_{2}$ ring spectrum with $\pi_{1}(A)=0$, and let $y: S^{d} \rightarrow$ $\Omega_{\otimes}^{\infty} A$, where $d \geq 0$ is even. Then the free $A_{\infty}$ based pre-log structure $\left(C_{1,0} S^{d}, \bar{y}_{0}\right)$ on $A$ generated by $y$ lifts to an $E_{2}$ based pre-log structure $(M, \alpha)$ on $A$, with $M \simeq C_{1,0} S^{d}$ and $\alpha$ homotopic to $\bar{y}_{0}$.

Example 9.16 Let $\left(C_{1,0} S^{2}, \bar{u}_{0}\right)$ be the free $A_{\infty}$ based pre-log structure on $k u_{(p)}$ generated by a map $S^{2} \rightarrow \Omega_{\otimes}^{\infty} k u_{(p)}$ representing $u \in \pi_{2} k u_{(p)}$, and let $\left(C_{1,0} S^{q}, \bar{v}_{1,0}\right)$ be the free $A_{\infty}$ based pre-log structure on $\ell$ generated by a map $S^{q} \rightarrow \Omega_{\otimes}^{\infty} \ell$ representing $v_{1} \in \pi_{q} \ell$. Let $f: \ell \rightarrow k u_{(p)}$ be the usual map of commutative $S$-algebras. The inclusion $S^{q}=S^{2(p-1)} \rightarrow C_{1,0} S^{2}$ to the $j=p-1$ summand extends to an $A_{\infty}$ map

$$
f^{b}: C_{1,0} S^{q} \rightarrow C_{1,0} S^{2}
$$


that makes $\left(f, f^{b}\right):\left(\ell, C_{1,0} S^{q}\right) \rightarrow\left(k u_{(p)}, C_{1,0} S^{2}\right)$ a map of $A_{\infty}$ based pre-log $S-$ algebras. Note that the natural map

$$
\ell \wedge \Sigma^{\infty} C_{1,0} S^{q} \Sigma^{\infty} C_{1,0} S^{2} \rightarrow k u_{(p)}
$$

is an equivalence, since the left hand side is equivalent to $\bigvee_{j=0}^{p-1} \Sigma^{2} \ell$, and compare with Lemma 12.15. Assuming some uniqueness or other compatibility of the $E_{2}$ lifts in Hypothesis 9.15, the map $\left(f, f^{\mathrm{b}}\right)$ can be promoted to be a map of $E_{2}$ based pre-log $S$-algebras. If so, $\operatorname{THH}\left(\Sigma^{\infty} C_{1,0} S^{q}\right)$ and $\operatorname{THH}\left(\Sigma^{\infty} C_{1,0} S^{2}\right)$ become $A_{\infty}$ ring spectra, we can construct $\operatorname{THH}\left(\ell, C_{1,0} S^{q}\right)$ and $\operatorname{THH}\left(k u_{(p)}, C_{1,0} S^{2}\right)$, and

$$
\operatorname{THH}\left(\ell, C_{1,0} S^{q}\right) \rightarrow \operatorname{THH}\left(k u_{(p)}, C_{1,0} S^{2}\right)
$$

becomes a map of $A_{\infty}$ ring spectra.

Remark 9.17 We summarize the results of these calculations. For $A=k u$ and $M=\langle p\rangle$, Lemmas 9.5, 9.6 and 9.7 consider the existence of $E_{n}$ pre-log structures $\alpha: M \rightarrow \Omega^{\infty} A_{\otimes}$ taking the monoid generator to the $p$-th component of $\Omega^{\infty} A_{\otimes}$. An $A_{\infty}=E_{1}$ pre-log structure certainly exists, and there is no homological obstruction to the existence of an $E_{2}$ pre-log structure, but no $E_{3}$ pre-log structure exists.

For $A=k u$ and $M=\bigvee_{j \geq 0} S^{2}$, Proposition 9.10 and Lemmas 9.12 and 9.13 concern $E_{n}$ pre-log structures $\alpha: M \rightarrow \Omega^{\infty} A_{\otimes}$ mapping $S^{2} \subset M$ to $\Omega^{\infty} A_{\otimes}$ to represent the Bott class $u \in \pi_{2} k u$. An $A_{\infty}=E_{1}$ pre-log structure certainly exists, and there is no homological obstruction to the existence of an $E_{2}$ pre-log structure, but no $E_{3}$ pre-log structure exists.

In Hypotheses 9.8 and 9.15, we propose a natural generality for the existence of $E_{2}$ pre-log structures. In Example 9.16, we discuss the consequences for the existence of a map $(\ell, M) \rightarrow\left(k u_{(p)}, N\right)$ of $E_{2}$ pre-log $S$-algebras, with $M \simeq \bigvee_{k \geq 0} S^{q k}$ and $N \simeq \bigvee_{j \geq 0} S^{2 j}$.

\section{Part III Logarithmic topological André-Quillen homology}

\section{Topological André-Quillen homology}

We now extend the construction of log Kähler forms and the log cotangent complex to the topological context.

Definition 10.1 Let $A$ be a commutative symmetric ring spectrum and let $J$ be a left $A$-module spectrum. Since $A$ is commutative, we can also think of $J$ as a right 
$A$-module. The square-zero extension $A \vee J$ is the commutative symmetric ring spectrum with multiplication map

$$
(A \vee J) \wedge(A \vee J) \cong(A \wedge A) \vee(A \wedge J) \vee(J \wedge A) \vee(J \wedge J) \rightarrow A \wedge J
$$

given by the multiplication $\mu: A \wedge A \rightarrow A$ on the first wedge summand, by the module actions $A \wedge J \rightarrow J$ and $J \wedge A \rightarrow J$ on the second and third summands, and by the trivial map $J \wedge J \rightarrow *$ on the fourth summand. We have maps

$$
A \stackrel{\eta}{\rightarrow} A \vee J \stackrel{\epsilon}{\rightarrow} A
$$

of commutative symmetric ring spectra, where $A$ is the unit inclusion and $\epsilon$ collapses $J$ to $*$. We think of $J$ as the kernel of $\epsilon$, making it a square-zero ideal in $A \vee J$.

Definition 10.2 Let $A$ be commutative symmetric ring spectrum, and let $J$ be an $A$-module. A derivation of $A$ with values in $J$ is a map $d: A \rightarrow A \vee J$ of commutative symmetric ring spectra over $A$. We let

$$
\operatorname{Der}_{S}(A, J)=\left(\mathcal{C S} p^{\Sigma} / A\right)(A, A \vee J)
$$

be the (homotopy invariant) mapping space of all such derivations.

More generally, for a map $e: R \rightarrow A$ of commutative symmetric ring spectra, we say that a derivation of $A$ over $R$ with values in $J$ is a map $d: A \rightarrow A \vee J$ of commutative symmetric ring spectra under $R$ and over $A$. It is a dashed arrow making the diagram

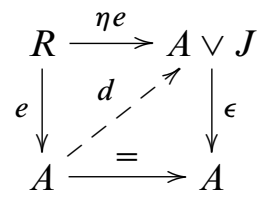

commute, in the category of commutative symmetric ring spectra. We let

$$
\operatorname{Der}_{R}(A, J)=\left(R / \mathcal{C S} p^{\Sigma} / A\right)(A, A \vee J)
$$

be the mapping space of all such derivations. We usually abbreviate $R / \mathcal{C} S p^{\Sigma}$ and $R / \mathcal{C S} p^{\Sigma} / A$ to $\mathcal{C}_{R}$ and $\mathcal{C}_{R} / A$, respectively.

Definition 10.3 The following definition is due to Maria Basterra [12]. The topological André-Quillen homology of $A$ over $R$ is the $A$-module

$$
\operatorname{TAQ}^{R}(A)=\operatorname{TAQ}(A / R)=\mathbb{L} Q_{A} \mathbb{R} I_{A}\left(A \wedge \wedge_{R}^{\mathbb{L}} A\right) .
$$

In other words, it is the homotopy invariant form of $Q_{A} I_{A}\left(A \wedge_{R} A\right)$. Here $A \wedge_{R} A$ is viewed as a commutative symmetric ring spectrum under and over $A$, via the left 
unit map id $\wedge e: A \cong A \wedge_{R} R \rightarrow A \wedge_{R} A$ and the multiplication $\mu: A \wedge_{R} A \rightarrow A$. The augmentation ideal functor $I_{A}: \mathcal{C}_{A} / A \rightarrow \mathcal{N}_{A}$, to the category of nonunital commutative $A$-algebras, is right adjoint to the functor $N \mapsto A \vee N$, and this adjoint pair forms a Quillen equivalence. The indecomposable quotient functor $Q_{A}: \mathcal{N}_{A} \rightarrow \mathcal{M}_{A}$, to the category of $A$-modules, is left adjoint to the functor that gives an $A$-module the trivial multiplication.

We say that $e: R \rightarrow A$ is formally étale if $\mathrm{TAQ}^{R}(A)$ is contractible. When $R=S$ is the sphere spectrum, we simply write $\operatorname{TAQ}(A)$ for $\operatorname{TAQ}^{S}(A)$.

Proposition 10.4 The topological André-Quillen homology corepresents derivations, in the sense that there is a natural weak equivalence

$$
\mathcal{M}_{A}\left(\mathrm{TAQ}^{R}(A), J\right) \simeq \operatorname{Der}_{R}(A, J)
$$

of homotopy invariant mapping spaces. There is a universal derivation

$$
d_{u}: A \rightarrow A \vee \mathrm{TAQ}^{R}(A)
$$

of $A$ over $R$ that corresponds to the identity map of $\mathrm{TAQ}^{R}(A)$.

Proof This is essentially Basterra's result $[12,3.2]$. By the (Quillen) adjunctions

$$
\mathcal{M}_{A} \stackrel{Q_{A}}{\longrightarrow} \mathcal{N}_{A} \stackrel{A \vee(-)}{\underset{I_{A}}{\longrightarrow}} \mathcal{C}_{A} / A
$$

one gets equivalences

$$
\mathcal{M}_{A}\left(\mathrm{TAQ}^{R}(A), J\right) \simeq \mathcal{N}_{A}\left(I_{A}\left(A \wedge_{R} A\right), J\right) \simeq\left(\mathcal{C}_{A} / A\right)\left(A \wedge_{R} A, A \vee J\right),
$$

and by the left hand pushout square in the diagram

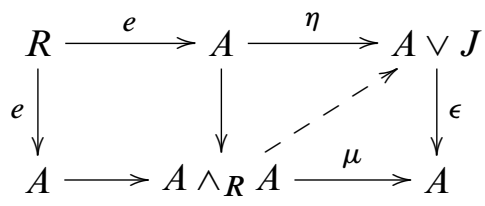

of commutative symmetric ring spectra, the dashed arrows correspond to derivations of $A$ over $R$ with values in $J$.

Remark 10.5 Implicit in Proposition 10.4 is the result that

$$
\operatorname{Der}_{R}(A, J) \simeq \Omega^{n} \operatorname{Der}_{R}\left(A, \Sigma^{n} J\right)
$$


is an infinite loop space, since $\mathcal{M}_{A}\left(\mathrm{TAQ}^{R}(A), J\right) \simeq \Omega^{n} \mathcal{M}_{A}\left(\mathrm{TAQ}^{R}(A), \Sigma^{n} J\right)$, for all $n \geq 0$. Hence the square-zero extension $A \vee J$ is an "infinite loop object" in $\mathcal{C}_{R} / A$, topologically analogous to the role of $A \oplus J$ as an abelian group object in $\mathcal{C} \mathcal{R} i n g / A$. See Remark 4.2.

Lemma 10.6 Let $g: C \rightarrow A$ be a map of commutative $R$-algebras, and let $J$ be an $A$-module. Write $g^{\#} J$ for $J$ viewed as a $C$-module via $g$. Composition with $g \vee$ id: $C \vee J \rightarrow A \vee J$ induces a weak equivalence

$$
\operatorname{Der}_{R}\left(C, g^{\#} J\right) \stackrel{\simeq}{\rightarrow}\left(\mathcal{C}_{R} / A\right)(C, A \vee J)
$$

Proof This follows since the right hand square in the diagram

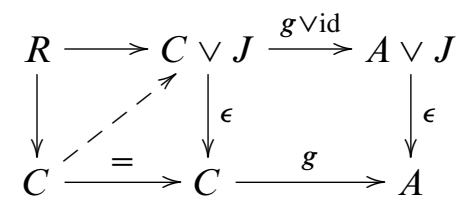

is a homotopy pullback.

Proposition 10.7 Let $R \stackrel{e}{\rightarrow} A \stackrel{f}{\rightarrow} B$ be maps of commutative symmetric ring spectra. There is a natural homotopy cofiber sequence

$$
B \wedge{ }_{A} \mathrm{TAQ}^{R}(A) \rightarrow \mathrm{TAQ}^{R}(B) \rightarrow \mathrm{TAQ}^{A}(B)
$$

of $B$-modules, known as the transitivity sequence for $e$ and $f$.

Proof See Basterra [12, 4.2] for this topological analogue of Quillen's work [60, 5.1].

Proposition 10.8 Let $e: R \rightarrow A$ and $g: R \rightarrow T$ be maps of commutative symmetric ring spectra. There is a natural weak equivalence

$$
T \wedge_{R} \mathrm{TAQ}^{R}(A) \stackrel{\simeq}{\rightarrow} \mathrm{TAQ}^{T}\left(T \wedge_{R} A\right)
$$

of $\left(T \wedge_{R} A\right)$-modules, known as flat base change along $g$.

Proof See Basterra $[12,4.6]$ for this topological analogue of Quillen's work [60, 5.3]. 
Lemma 10.9 Let $M=C X=\bigsqcup_{j \geq 0} X^{\otimes j} / \Sigma_{j}$ be the free commutative $\mathcal{I}$-space monoid on an $\mathcal{I}$-space $X$, so that $S[M]=P S[X]=\bigvee_{j \geq 0} S[X]^{\wedge j} / \Sigma_{j}$ is the free commutative symmetric ring spectrum on the symmetric spectrum $S[X]$. Then

$$
\operatorname{TAQ}(S[M]) \simeq S[M] \wedge S[X]
$$

and the universal derivation $d_{u}: S[M] \rightarrow S[M] \vee(S[M] \wedge S[X])$ is the commutative symmetric ring spectrum map that extends the symmetric spectrum map

$$
i \vee(\eta \wedge \mathrm{id}): S[X] \rightarrow S[M] \vee(S[M] \wedge S[X])
$$

given as the wedge sum of the inclusion $i: S[X] \rightarrow S[M]$ and the unit map $\eta \wedge$ id: $S[X] \cong S \wedge S[X] \rightarrow S[M] \wedge S[X]$.

Similarly, let $N=C_{0} Y=\bigvee_{j \geq 0} Y^{\square j} / \Sigma_{j}$ be the free commutative based $\mathcal{I}$-space monoid on a based $\mathcal{I}$-space $Y$, so that $\Sigma^{\bullet} N=P \Sigma^{\bullet} Y$ is the free commutative symmetric ring spectrum on the symmetric spectrum $\Sigma^{\bullet} Y$. Then

$$
\operatorname{TAQ}\left(\Sigma^{\bullet} N\right) \simeq \Sigma^{\bullet} N \wedge \Sigma^{\bullet} Y
$$

and the universal derivation $d_{u}: \Sigma^{\bullet} N \rightarrow \Sigma^{\bullet} N \vee\left(\Sigma^{\bullet} N \wedge \Sigma^{\bullet} Y\right)$ is the commutative symmetric ring spectrum map that extends the symmetric spectrum map

$$
i \vee(\eta \wedge \mathrm{id}): \Sigma^{\bullet} Y \rightarrow \Sigma^{\bullet} N \vee\left(\Sigma^{\bullet} N \wedge \Sigma^{\bullet} Y\right)
$$

given as the wedge sum of the inclusion $i: \Sigma^{\bullet} Y \rightarrow \Sigma^{\bullet} N$ and the unit map $\eta \wedge$ id: $\Sigma^{\bullet} N \cong S \wedge \Sigma^{\bullet} Y \rightarrow \Sigma^{\bullet} N \wedge \Sigma^{\bullet} Y$.

Proof For each $S[M]$-module $J$, the space $\operatorname{Der}(S[M], J)$ of dashed maps

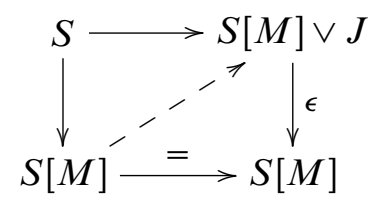

in the category of commutative symmetric ring spectra is equivalent to the space of dashed maps

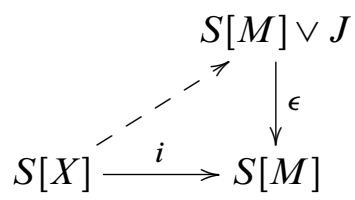

in the category of symmetric spectra, which by projection along $p: S[M] \vee J \rightarrow J$ is equivalent to the space of symmetric spectrum maps $S[X] \rightarrow J$. In particular, these are 
corepresented by the symmetric spectrum $S[X]$, and by the induced $S[M]$-module $\operatorname{TAQ}(S[M])=S[M] \wedge S[X]$.

The universal derivation $d_{u}$ corresponds to the identity on $S[M] \wedge S[X]$ as an $S[M]-$ module map, which corresponds to $\eta \wedge \mathrm{id}: S[X] \rightarrow S[M] \wedge S[X]$ as a symmetric spectrum map, and to the multiplicative extension of the map $i \vee(\eta \wedge \mathrm{id}): S[X] \rightarrow$ $S[M] \vee(S[M] \wedge S[X])$ as a symmetric ring spectrum map over $S[M]$.

The proof in the based case is identical.

Remark 10.10 For more general commutative $\mathcal{I}$-space monoids $M$, built as cell complexes by attaching copies of $C X$ along $C A$ for suitable $\mathcal{I}$-spaces $A \subset X$, one can inductively compute $\operatorname{TAQ}(S[M])$ by combining Propositions 10.7 and 10.8 with Lemma 10.9. For example, if $N$ is a $\mathrm{CW}$ complex in commutative based $\mathcal{I}$-space monoids, so that the $k$-skeleton $N_{k}$ is obtained from $N_{k-1}$ by attaching $C X$ along $C A$ for $X \simeq \bigvee D^{k}$ and $A \simeq \bigvee S^{k-1}$, then there is a homotopy cofiber sequence

$$
\Sigma^{\bullet} N \wedge \Sigma^{\bullet} N_{k-1} \operatorname{TAQ}\left(\Sigma^{\bullet} N_{k-1}\right) \rightarrow \Sigma^{\bullet} N \wedge_{\Sigma} N_{k} \operatorname{TAQ}\left(\Sigma^{\bullet} N_{k}\right) \rightarrow \Sigma^{\bullet} N \wedge \bigvee S^{k}
$$

of $\Sigma^{\bullet} N$-modules. The homotopy colimit

$$
\operatorname{TAQ}\left(\Sigma^{\bullet} N\right)=\underset{k}{\operatorname{hocolim}}\left(\Sigma^{\bullet} N \wedge \Sigma^{\bullet} N_{k} \operatorname{TAQ}\left(\Sigma^{\bullet} N_{k}\right)\right)
$$

can then be assembled from the filtration quotients $\Sigma^{\bullet} N \wedge \bigvee S^{k}$, in the usual manner known from cellular homology and the Atiyah-Hirzebruch spectral sequence; see Baker-Gilmour-Reinhard [11].

Remark 10.11 For grouplike $E_{\infty}$ spaces $M$, Basterra and Mandell [13, Theorem 5] prove that $\mathrm{TAQ}(S[M]) \simeq S[M] \wedge B^{\infty} M$ as an extended $S[M]$-module. The condition that $M$ is grouplike is omitted in the published statement, but was needed for their intended argument, as Mike Mandell has kindly pointed out. We therefore reproduce part of their argument here, to show where the grouplike hypothesis is needed.

The shear map $\Gamma M \times M \rightarrow \Gamma M \times M$, given on elements by $(m, n) \mapsto(m \gamma(n), n)$, induces a weak equivalence

$$
S[\Gamma M] \wedge S[M] \rightarrow S[\Gamma M] \wedge S[M]
$$

of commutative $S[\Gamma M]$-algebras. This is a map of augmented $S[\Gamma M]$-algebras, where the augmentation on the left hand side is induced by the multiplication $\Gamma M \times M \rightarrow$ $\Gamma M \times \Gamma M \rightarrow \Gamma M$, and the augmentation on the right hand side is induced by the projection $\Gamma M \times M \rightarrow \Gamma M \times * \cong \Gamma M$ that collapses $M$ to $*$. By [13, Theorem 6.1] the commutative $S[\Gamma M]$-algebra indecomposables of the two sides are 
$S[\Gamma M] \wedge S[M] \operatorname{TAQ}(S[M])$ and the extended module $S[\Gamma M] \wedge B^{\infty} M$, respectively. Hence $\operatorname{TAQ}(S[M]) \simeq S[M] \wedge B^{\infty} M$ when $M$ is grouplike, but for general $M$ this only holds after base change along $S[M] \rightarrow S[\Gamma M]$.

Since pre-log structures mapping $M$ into $\mathrm{GL}_{1}(A)$ only give rise to trivial log structures, the Basterra-Mandell result for grouplike $M$ is not directly relevant to our discussion. Also the extended version is of modest direct use, since a pre-log $S-$ algebra $(A, M)$ becomes log trivial after base change to $(A \wedge S[M] S[\Gamma M], M)$ or $(A \wedge S[M] S[\Gamma M], \Gamma M)$. However, a slightly modified version of the shear map above is of fundamental importance in the general description of repletion maps given in Section 13 below.

\section{Logarithmic topological André-Quillen homology}

Definition 11.1 Define the grouplike commutative $\mathcal{I}$-space monoid $\left(1+\Omega^{\bullet} J\right)_{\otimes}$ by the homotopy fiber sequence

$$
\left(1+\Omega^{\bullet} J\right)_{\otimes} \rightarrow \mathrm{GL}_{1}(A \vee J) \stackrel{\mathrm{GL}_{1}(\epsilon)}{\longrightarrow} \mathrm{GL}_{1}(A)
$$

where $\mathrm{GL}_{1}(\epsilon)$ is split by $\mathrm{GL}_{1}(\eta)$. More explicitly, its $n$-th space is the homotopy fiber at $\eta_{n}: S^{n} \rightarrow A_{n}$ of the projection $\Omega^{n}\left(A_{n} \vee J_{n}\right) \rightarrow \Omega^{n} A_{n}$. We get a weak equivalence

$$
\mathrm{GL}_{1}(A) \otimes\left(1+\Omega^{\bullet} J\right)_{\otimes} \stackrel{\simeq}{\rightarrow} \mathrm{GL}_{1}(A \vee J)
$$

(for semistable $A$ and $J$ ) expressing $\mathrm{GL}_{1}(A \vee J)$ as the homotopy coproduct of $\mathrm{GL}_{1}(A)$ and $\left(1+\Omega^{\bullet} J\right)_{\otimes}$.

Lemma 11.2 The projection $A \vee J \rightarrow J$ induces a weak equivalence

$$
\left(1+\Omega^{\bullet} J\right)_{\otimes} \stackrel{\simeq}{\rightarrow} \Omega^{\bullet} J
$$

of $\mathcal{I}$-spaces, which is compatible up to preferred homotopy with the grouplike $E_{\infty}$ structures on $\left(1+\Omega^{\bullet} J\right)_{\otimes, h \mathcal{I}}$ and $\left(\Omega^{\bullet} J\right)_{h \mathcal{I}}$. Hence the spectrum associated to the commutative $\mathcal{I}$-space monoid $\left(1+\Omega^{\bullet} J\right)_{\otimes}$ is weakly equivalent to the underlying spectrum of $J$.

Proof The inclusion $A \vee J \rightarrow A \times J$ is a stable equivalence, so the map of homotopy fibers

$$
\left(1+\Omega^{\bullet} J\right)_{\otimes} \rightarrow \Omega^{\bullet} J
$$

(for the projections to $A$ ) is also a weak equivalence. 
For brevity, write $F_{n}$ for the homotopy fiber of $\Omega^{n}\left(A_{n} \vee J_{n}\right) \rightarrow \Omega^{n} A^{n}$ at $\eta_{n}$. Given a pinch map $S^{m+n} \rightarrow S^{m+n} \vee S^{m+n}$, the composite $F_{m} \times F_{n} \rightarrow F_{m+n} \rightarrow \Omega^{m+n} J_{m+n}$ has a preferred homotopy to the composite

$$
F_{m} \times F_{n} \rightarrow \Omega^{m} J_{m} \times \Omega^{n} J_{n} \rightarrow \Omega^{m+n} J_{m+n} \times \Omega^{m+n} J_{m+n} \rightarrow \Omega^{m+n} J_{m+n},
$$

where the middle map is the product of the stabilization maps in $J$, and the right hand map is the loop sum specified by the pinch map. Parametrizing the pinch maps by pairs of little $(m+n)$-cubes, and generalizing to products with more than two factors, we get the desired equivalence of $E_{\infty}$ structures.

Remark 11.3 An alternative argument in the language of $\mathcal{L}$-spaces can be given using [13, Theorem 6.1]. Basterra and Mandell construct a weak equivalence of $S$-modules $\mathbb{L} Q_{S} \mathbb{R} I_{S} S\left[\Omega^{\infty} J\right] \rightarrow J$, which is left adjoint to a map $\mathbb{R} I_{S} S\left[\Omega^{\infty} J\right] \rightarrow J$ of nonunital commutative $S$-algebras, which in turn is equivalent to a map $S\left[\Omega^{\infty} J\right] \rightarrow S \vee J$ of commutative $S$-algebras over $S$, which finally is left adjoint to a map of grouplike $\mathcal{L}$-spaces from $\Omega^{\infty} J$ to the homotopy fiber of $\mathrm{GL}_{1}(S \vee J) \rightarrow \mathrm{GL}_{1}(S)$, and the latter is equivalent to $\left(1+\Omega^{\infty} J\right)_{\otimes}$. This map of grouplike $\mathcal{L}$-spaces is the desired weak equivalence.

Definition 11.4 Let $(M, \alpha)$ be a log structure on $A$. The inverse image log structure $\left(\eta^{*} M, \eta^{*} \alpha\right)$ on $A \vee J$ is given by the upper central pushout square in the following diagram of commutative $\mathcal{I}$-space monoids.

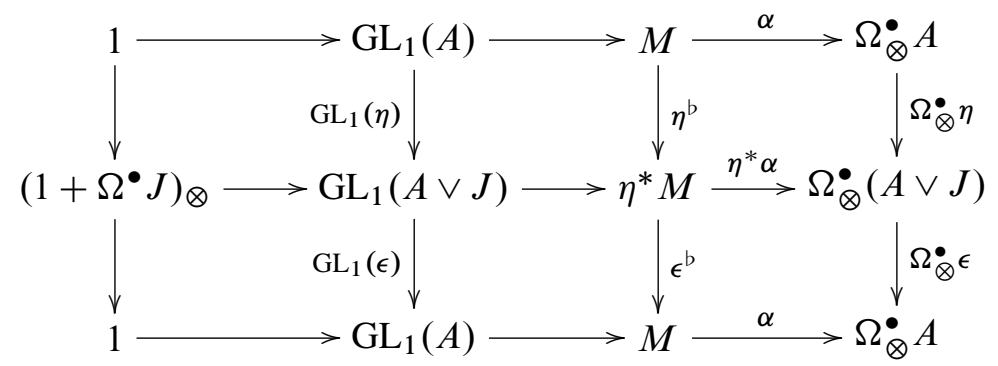

Since $\epsilon \eta=$ id we can identify $\epsilon^{*} \eta^{*} M$ with $M$.

Lemma 11.5 There is a chain of weak equivalences

$$
M \times\left(1+\Omega^{\bullet} J\right)_{\otimes} \stackrel{\simeq}{\longleftarrow} M \otimes\left(1+\Omega^{\bullet} J\right)_{\otimes} \stackrel{\simeq}{\rightarrow} \eta^{*} M
$$

and $\eta^{*} \alpha$ maps $(m, 1+j)$ to $\alpha(m) \cdot(1+j)=\alpha(m)+\alpha(m) j$. The natural maps $\eta^{b}$ and $\epsilon^{b}$ correspond to the product of the identity on $M$ with the base point inclusion and collapse maps $1 \rightarrow\left(1+\Omega^{\bullet} J\right)_{\otimes} \rightarrow 1$, respectively. 
Proof The upper left hand square in Definition 11.4 is a homotopy pushout, hence so is the rectangle with vertices $1, M,\left(1+\Omega^{\bullet} J\right)_{\otimes}$ and $\eta^{*} M$.

Definition 11.6 When $(M, \alpha)$ is a pre-log structure on $A$, we define

$$
\eta^{*} M=M \otimes\left(1+\Omega^{\bullet} J\right)_{\otimes} .
$$

In view of Lemma 11.2, there is a weak equivalence $\eta^{*} M \simeq M \times \Omega^{\bullet} J$. We define the pre-log structure

$$
\eta^{*} \alpha: \eta^{*} M \rightarrow \Omega_{\otimes}^{\bullet}(A \vee J)
$$

as the coproduct in commutative $\mathcal{I}$-space monoids of the pre-log structure $\Omega_{\otimes}^{\bullet} \eta \circ$ $\alpha: M \rightarrow \Omega_{\otimes}^{\bullet}(A \vee J)$ and the composite

$$
i_{J}:\left(1+\Omega^{\bullet} J\right)_{\otimes} \rightarrow \mathrm{GL}_{1}(A \vee J) \stackrel{\iota}{\rightarrow} \Omega_{\otimes}^{\bullet}(A \vee J)
$$

There results a commutative diagram

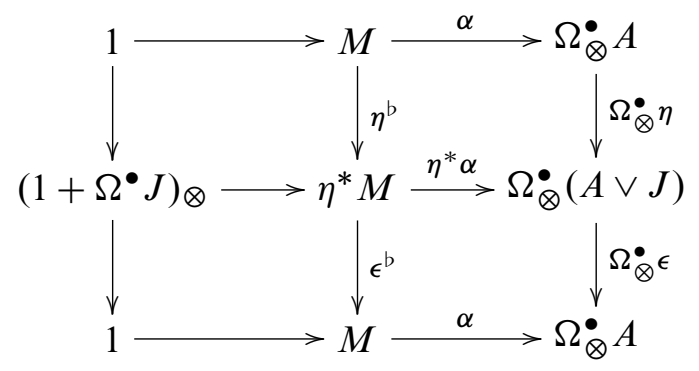

of commutative $\mathcal{I}$-space monoids, where the two left hand squares are pushouts.

Lemma 11.7 Let $(M, \alpha)$ be a pre-log structure on $A$, and $J$ an $A$-module. There is an equivalence

$$
\left(\eta^{*} M\right)^{a} \simeq \eta^{*}\left(M^{a}\right)
$$

of $\log$ structures in $A \vee J$.

Proof The proof is similar to that for Lemma 7.24.

Definition 11.8 Let $(A, M)$ be a pre-log symmetric ring spectrum, and let $J$ be an $A$-module. A $\log$ derivation of $(A, M)$ with values in $J$ is a map

$$
\left(d, d^{b}\right):(A, M) \rightarrow\left(A \vee J, \eta^{*} M\right)
$$

of pre-log symmetric ring spectra over $(A, M)$. Let

$$
\operatorname{Der}_{S}((A, M), J)=(\operatorname{Pre} \log (S) /(A, M))\left((A, M),\left(A \vee J, \eta^{*} M\right)\right)
$$

be the mapping space of all such log derivations. 
More generally, for a map $\left(e, e^{b}\right):(R, P) \rightarrow(A, M)$ of pre-log symmetric ring spectra we say that a $\log$ derivation of $(A, M)$ over $(R, P)$ with values in the $A$-module $J$ is a map

$$
\left(d, d^{b}\right):(A, M) \rightarrow\left(A \vee J, \eta^{*} M\right)
$$

of pre-log symmetric ring spectra under $(R, P)$ and over $(A, M)$. In other words, it is a dashed arrow making the diagram of pre-log symmetric ring spectra

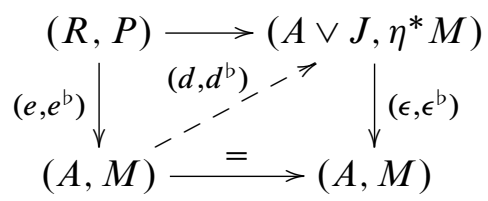

commute. The top horizontal map is $\left(\eta, \eta^{b}\right) \circ\left(e, e^{b}\right)=\left(\eta e, \eta^{b} e^{b}\right)$. We let

$$
\operatorname{Der}_{(R, P)}((A, M), J)=(\mathcal{P r e \mathcal { L } o g}(R, P) /(A, M))\left((A, M),\left(A \vee J, \eta^{*} M\right)\right)
$$

be the mapping space of all such log derivations.

Lemma 11.9 The logification maps $(R, P) \rightarrow\left(R, P^{a}\right)$ and $(A, M) \rightarrow\left(A, M^{a}\right)$ induce weak equivalences

$$
\operatorname{Der}_{\left(R, P^{a}\right)}\left(\left(A, M^{a}\right), J\right) \stackrel{\simeq}{\rightarrow} \operatorname{Der}_{(R, P)}\left(\left(A, M^{a}\right), J\right) \stackrel{\simeq}{\rightarrow} \operatorname{Der}_{(R, P)}((A, M), J) .
$$

Proof Let $Z$ be the space of dashed arrows making the diagram

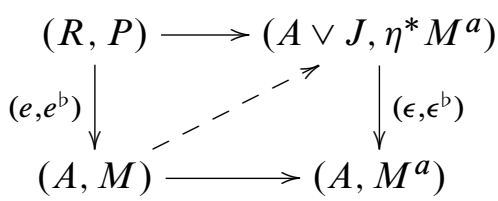

commute, in the category of pre-log symmetric ring spectra. The first map of the lemma, and the natural map $\operatorname{Der}_{(R, P)}\left(\left(A, M^{a}\right), J\right) \rightarrow Z$, are weak equivalences by Lemma 7.8, since we are considering spaces of pre-log maps into the log symmetric ring spectra $\left(A \vee J, \eta^{*} M^{a}\right)$ and $\left(A, M^{a}\right)$. The natural map $\operatorname{Der}_{(R, P)}((A, M), J) \rightarrow Z$ is also a weak equivalence, because

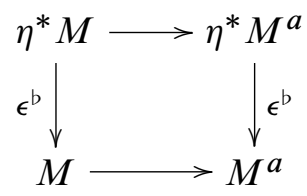

is a homotopy pullback square. Hence the second map in the lemma is also a weak equivalence. 
Definition 11.10 Let $M$ and $K$ be commutative $\mathcal{I}$-space monoids, with $K$ grouplike. The space of commutative $\mathcal{I}$-space monoid derivations of $M$ with values in $K$ is the mapping space

$$
\operatorname{Der}^{b}(M, K)=\left(\mathcal{C S}^{\mathcal{I}} / M\right)(M, M \times K)
$$

of commutative $\mathcal{I}$-space monoid homomorphisms $d^{\mathrm{b}}: M \rightarrow M \times K$ over $M$.

More generally, let $e^{b}: P \rightarrow M$ be a map of commutative $\mathcal{I}$-space monoids. The space

$$
\operatorname{Der}_{P}^{\mathrm{b}}(M, K)=\left(P / \mathcal{C S}^{\mathcal{I}} / M\right)(M, M \times K)
$$

of commutative $\mathcal{I}$-space monoid derivations of $M$ over $P$ with values in $K$ is the space of dashed arrows $d^{b}$ making the diagram

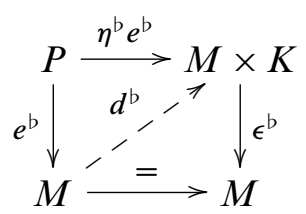

of commutative $\mathcal{I}$-space monoids commute.

Lemma 11.11 There are natural equivalences

$$
\begin{aligned}
\operatorname{Der}^{b}(M, K) & \simeq \mathcal{C S}^{\mathcal{I}}(M, K) \simeq \mathcal{S} p^{\Sigma}\left(B^{\infty} M, B^{\infty} K\right) \\
\operatorname{Der}_{P}^{b}(M, K) & \simeq \mathcal{S} p^{\Sigma}\left(B^{\infty} M / B^{\infty} P, B^{\infty} K\right)
\end{aligned}
$$

The universal commutative $\mathcal{I}$-space monoid derivation

$$
d_{u}^{b}: M \rightarrow M \times \Gamma M
$$

of $M$ corresponds to the identity map of $B^{\infty} M$, and is given by the composite

$$
M \stackrel{\Delta}{\rightarrow} M \times M \stackrel{\text { id } \times \gamma}{\longrightarrow} M \times \Gamma M .
$$

More generally, the universal commutative $\mathcal{I}$-space monoid derivation

$$
d_{u}^{\text {b }}: M \rightarrow M \times \Omega^{\bullet}\left(B^{\bullet} M / B^{\bullet} P\right)
$$

of $M$ over $P$ corresponds to the identity map of $B^{\infty} M / B^{\infty} P$.

Proof It is clear that commutative $\mathcal{I}$-space monoid homomorphisms $d^{\text {b }}: M \rightarrow M \times K$ under $P$ and over $M$ correspond to commutative $\mathcal{I}$-space monoid homomorphisms 
$M \rightarrow K$ that take $P$ to $*$.

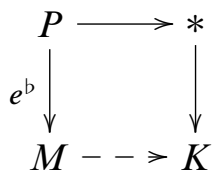

Since $K$ is grouplike, the latter are equivalent to maps $B^{\infty} M \rightarrow B^{\infty} K$ of symmetric spectra, that come with a nullhomotopy of the restriction to $B^{\infty} P$. These are in turn equivalent to maps from the homotopy cofiber of $B^{\infty} P \rightarrow B^{\infty} M$, which we write as $B^{\infty} M / B^{\infty} P$.

Remark 11.12 Implicit in Lemma 11.11 is the result that

$$
\operatorname{Der}^{\mathrm{b}}(M, K) \simeq \Omega^{n} \operatorname{Der}^{\mathrm{b}}\left(A, B^{n} K\right)
$$

is an infinite loop space, since $\mathcal{S} p^{\Sigma}\left(B^{\infty} M, B^{\infty} K\right) \simeq \Omega^{n} \mathcal{S} p^{\Sigma}\left(B^{\infty} M, B^{\infty} B^{n} K\right)$, for all $n \geq 0$, and similarly for nontrivial $P$. The product $M \times K$ is an "infinite loop object" in $\mathcal{C S}^{\mathcal{I}} / M$.

Proposition 11.13 Let $\left(e, e^{b}\right):(R, P, \rho) \rightarrow(A, M, \alpha)$ be a map of pre-log symmetric ring spectra, and let $J$ be an $A$-module. There is a homotopy pullback square

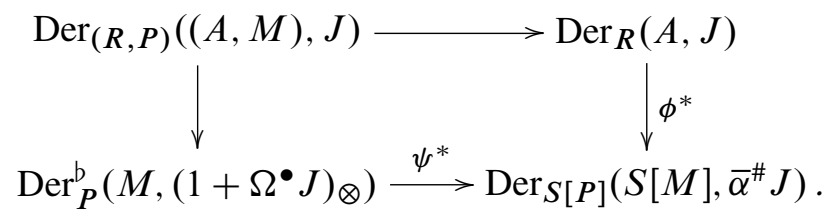

Proof A $\log$ derivation $\left(d, d^{b}\right)$ as in diagram (11-1) is equivalent to a pair of $\log$ derivations $d$ and $d^{\mathrm{b}}$, as in diagrams (10-1) and (11-2), respectively, subject to the compatibility condition $\Omega_{\otimes}^{\bullet} d \circ \alpha=\eta^{*} \alpha \circ d^{\mathrm{b}}$ in the space of dashed arrows

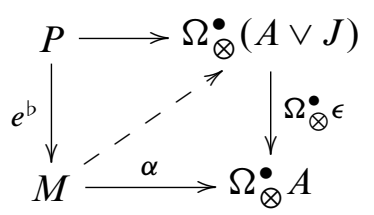

making this a commutative diagram in $\mathcal{C S}^{\mathcal{I}}$, or equivalently, in the space of dashed arrows

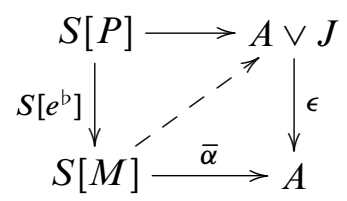


making this a commutative diagram in $\mathcal{C S} p^{\Sigma}$. The upper horizontal arrows are $\left(\Omega_{\otimes}^{\bullet} \eta\right) \circ \alpha \circ e^{b}$ and $\eta \circ \bar{\alpha} \circ S\left[e^{b}\right]$, respectively. By Lemma 10.6, the latter space is weakly equivalent to $\operatorname{Der}_{S[P]}\left(S[M], \bar{\alpha}^{\#} J\right)$, since there is a homotopy pullback square

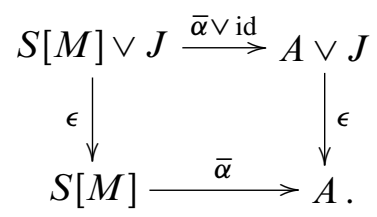

The map $\phi^{*}$ takes a derivation $d$ of $A$ over $R$ with values in $J$ to $\Omega_{\otimes}^{\bullet} d \circ \alpha$, which under these identifications corresponds to $d \circ \bar{\alpha}: S[M] \rightarrow A \vee J$ and its lift (up to contractible choice) to a derivation of $S[M]$.

The map $\psi^{*}$ takes a commutative $\mathcal{I}$-space monoid derivation $d^{\mathrm{b}}$ of $M$ over $P$ with values in $\left(1+\Omega^{\bullet} J\right)_{\otimes}$ to $\eta^{*} \alpha \circ d^{\mathrm{b}}$, which corresponds to $\overline{\eta^{*} \alpha} \circ S\left[d^{\mathrm{b}}\right]: S[M] \rightarrow A \vee J$, and to its lift to a derivation of $S[M]$.

Remark 11.14 In view of Remarks 10.5 and 11.12,

$$
\operatorname{Der}_{(R, P)}((A, M), J) \simeq \Omega^{n} \operatorname{Der}_{(R, P)}\left((A, M), \Sigma^{n} J\right)
$$

for all $n \geq 0$, so the square-zero extensions $\left(A \vee J, \eta^{*} M\right)$ are infinite loop objects in $\log (S) /(A, M)$.

Lemma 11.15 Let $\left(e, e^{b}\right):(R, P, \rho) \rightarrow(A, M, \alpha)$ be a map of pre-log symmetric ring spectra. The functors from $A$-modules to (infinite loop) spaces that take $J$ to $\operatorname{Der}_{R}(A, J)$ and $\operatorname{Der}_{S[P]}\left(S[M], \bar{\alpha}^{\#} J\right)$ are corepresented by the $A$-modules $\operatorname{TAQ}^{R}(A)$ and $A \wedge S[M] \mathrm{TAQ}^{S[P]}(S[M])$, respectively. The natural map $\phi^{*}$ is corepresented by the map

$$
\phi: A \wedge S[M] \mathrm{TAQ}^{S[P]}(S[M]) \rightarrow \mathrm{TAQ}^{R}(A)
$$

of $A$-modules, induced by the maps $\bar{\rho}: S[P] \rightarrow R$ and $\bar{\alpha}: S[M] \rightarrow A$ of commutative symmetric ring spectra. For $(R, P)=(S, 1)$, it is left adjoint to the $S[M]$-module map $\operatorname{TAQ}(S[M]) \rightarrow \operatorname{TAQ}(A)$ induced by $\bar{\alpha}$.

Proof The functor $J \mapsto \operatorname{Der}_{R}(A, J)$ is corepresented by $\operatorname{TAQ}^{R}(A)$, by Proposition 10.4. The functor $K \mapsto \operatorname{Der}_{S[P]}(S[M], K)$, from $S[M]$-modules, is corepresented by $\mathrm{TAQ}^{S[P]}(S[M])$, hence its composite with $J \mapsto \bar{\alpha}^{\#} J$ is corepresented by the base change $A \wedge S[M] \mathrm{TAQ}^{S[P]}(S[M])$.

Modulo the identifications given by Lemma 10.6, the map $\phi^{*}$ is given by composition with $\bar{\alpha}$, as discussed at the end of the proof of Proposition 11.13. Hence the corepresenting map $\phi$ is also induced by $\bar{\alpha}$. 
Lemma 11.16 Let $\left(e, e^{b}\right):(R, P, \rho) \rightarrow(A, M, \alpha)$ be a map of pre-log symmetric ring spectra. The functors from $A$-modules to (infinite loop) spaces that take $J$ to $\operatorname{Der}_{P}^{b}\left(M,\left(1+\Omega^{\bullet} J\right)_{\otimes}\right)$ and $\operatorname{Der}_{S[P]}\left(S[M], \bar{\alpha}^{\#} J\right)$ are corepresented by the $A-$ modules $A \wedge\left(B^{\infty} M / B^{\infty} P\right)$ and $A \wedge S[M] \mathrm{TAQ}^{S[P]}(S[M])$, respectively. The natural map $\psi^{*}$ is corepresented by a map

$$
\psi: A \wedge S[M] \operatorname{TAQ}^{S[P]}(S[M]) \rightarrow A \wedge\left(B^{\infty} M / B^{\infty} P\right)
$$

of $A$-modules.

Proof By Lemmas 11.2 and 11.11 we have a natural chain of equivalences

$$
\begin{aligned}
\operatorname{Der}_{P}^{\mathrm{b}}\left(M,\left(1+\Omega^{\bullet} J\right)_{\otimes}\right) & \simeq \operatorname{Der}_{P}^{\mathrm{b}}\left(M, \Omega^{\bullet} J\right) \\
& \simeq \mathcal{S} p^{\Sigma}\left(B^{\infty} M / B^{\infty} P, J\right) \simeq \mathcal{M}_{A}\left(A \wedge\left(B^{\infty} M / B^{\infty} P\right), J\right) .
\end{aligned}
$$

Hence $A \wedge\left(B^{\infty} M / B^{\infty} P\right)$ corepresents the first functor. The existence of a corepresenting map follows from the Yoneda lemma.

Proposition 11.17 Let $(A, M, \alpha)$ be a pre-log symmetric ring spectrum, and assume that $M=C X$ is the free commutative $\mathcal{I}$-space monoid on an $\mathcal{I}$-space $X$. Let $\bar{\alpha}_{X}=\bar{\alpha} \circ i: S[X] \rightarrow A$ be the restriction of the adjoint structure map $\bar{\alpha}: S[M] \rightarrow A$ over the inclusion $i: S[X] \rightarrow S[M]$. The corepresenting map

$$
\psi: A \wedge S[M] \operatorname{TAQ}(S[M]) \rightarrow A \wedge B^{\infty} M
$$

factors as

$$
\begin{aligned}
A \wedge S[M] \mathrm{TAQ}(S[M]) & \simeq A \wedge S[X] \\
& \stackrel{\text { id } \wedge S[\Delta]}{\longrightarrow} A \wedge S[X \times X] \simeq A \wedge S[X] \wedge S[X] \\
& \stackrel{\text { id } \wedge \bar{\alpha}_{X} \wedge \mathrm{id}}{\longrightarrow} A \wedge A \wedge S[X] \\
& \stackrel{\mu \wedge \text { id }}{\longrightarrow} A \wedge S[X] \simeq A \wedge B^{\infty} M
\end{aligned}
$$

where $\Delta: X \rightarrow X \times X$ is the $\mathcal{I}$-space diagonal and $\mu: A \wedge A \rightarrow A$ is the symmetric ring spectrum product.

Proof The first and last weak equivalences follow from Lemmas 10.9 and 6.22, respectively.

To identify the $A$-module map $\psi$, we view it as corepresenting a derivation $d$ of $S[M]$ with values in the underlying $S[M]$-module $\bar{\alpha}^{\#} J$ of the extended $A$-module $J=A \wedge B^{\infty} M$, as in diagram (11-4). We fix this value of $J$ for the rest of the 
proof. By adjunction, such a derivation corresponds to a commutative $\mathcal{I}$-space monoid map $M \rightarrow \Omega_{\otimes}^{\bullet}(A \vee J)$ over $\Omega_{\otimes}^{\bullet} A$, as in diagram (11-3). The relevant commutative $\mathcal{I}$-space monoid map is the composite $\eta^{*} \alpha \circ d^{\mathrm{b}}$ in the following diagram

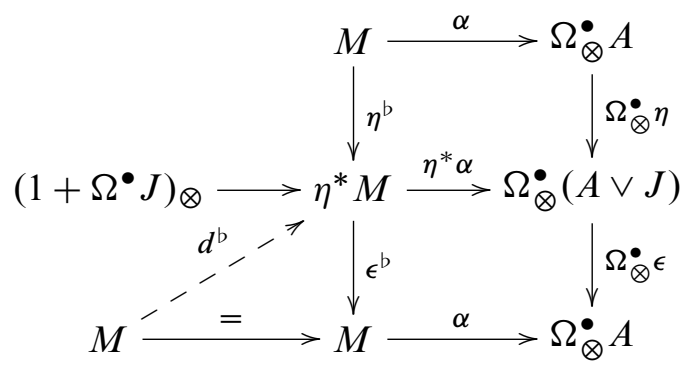

of commutative $\mathcal{I}$-space monoids. Here $d^{\text {b }}$ is the map that is corepresented by the identity map of $J=A \wedge B^{\infty} M$, more-or-less as in Lemma 11.11. Modulo the weak equivalence

$$
\eta^{*} M=M \otimes\left(1+\Omega^{\bullet} J\right)_{\otimes} \stackrel{\simeq}{\rightarrow} M \times\left(1+\Omega^{\bullet} J\right)_{\otimes}
$$

(for reasonable $M$ and $J$ ), we can write $d^{\mathrm{b}}$ as the composite

$$
M \stackrel{\Delta}{\rightarrow} M \times M \stackrel{\operatorname{id} \times\left(1+\gamma^{\prime}\right)}{\longrightarrow} M \times\left(1+\Omega^{\bullet} J\right)_{\otimes}
$$

where $\left(1+\gamma^{\prime}\right)$ is the composite

$$
M \stackrel{\gamma}{\rightarrow} \Omega^{\bullet} B^{\bullet} M \stackrel{\Omega^{\bullet}\left(\eta_{A} \wedge \text { id }\right)}{\longrightarrow} \Omega^{\bullet}\left(A \wedge B^{\bullet} M\right)=\Omega^{\bullet} J \simeq\left(1+\Omega^{\bullet} J\right)_{\otimes} .
$$

Here $\gamma$ is the group completion map, $\eta_{A}: S \rightarrow A$ is the unit map for $A$, and the last equivalence uses Lemma 11.2.

The map $\eta^{*} \alpha$ is the coproduct of the map $\Omega_{\otimes}^{\bullet} \eta \circ \alpha$ and the inclusion

$$
i_{J}:\left(1+\Omega^{\bullet} J\right)_{\otimes} \rightarrow \Omega_{\otimes}^{\bullet}(A \vee J),
$$

hence can be written as the composite

$$
M \otimes\left(1+\Omega^{\bullet} J\right)_{\otimes} \stackrel{\alpha \otimes i_{J}}{\longrightarrow} \Omega_{\otimes}^{\bullet} A \otimes \Omega_{\otimes}^{\bullet}(A \vee J) \stackrel{\lambda^{\prime}}{\longrightarrow} \Omega_{\otimes}^{\bullet}(A \vee J)
$$

where $\lambda^{\prime}$ is the pairing induced from the $A$-module action on $A \vee J$. The composite $\eta^{*} \alpha \circ d^{b}$ therefore factors as

$$
M \stackrel{\Delta}{\rightarrow} M \times M \simeq M \otimes M \stackrel{\alpha \otimes i_{J}\left(1+\gamma^{\prime}\right)}{\longrightarrow} \Omega_{\otimes}^{\bullet} A \otimes \Omega_{\otimes}^{\bullet}(A \vee J) \stackrel{\lambda^{\prime}}{\longrightarrow} \Omega_{\otimes}^{\bullet}(A \vee J)
$$


Passing to left adjoints, we find that the derivation $d$ corepresented by $\psi$ is the composite map

$$
d: S[M] \stackrel{S[\Delta]}{\longrightarrow} S[M \times M] \simeq S[M] \wedge S[M] \stackrel{\bar{\alpha} \wedge\left(1+\epsilon^{\prime}\right)}{\longrightarrow} A \wedge(A \vee J) \stackrel{\lambda}{\rightarrow} A \vee J
$$

where $\left(1+\epsilon^{\prime}\right)$ is the composite

$$
S[M] \stackrel{\epsilon}{\rightarrow} B^{\infty} M \stackrel{\eta_{A} \wedge \mathrm{id}}{\longrightarrow} A \wedge B^{\infty} M=J \rightarrow A \vee J
$$

using the unit of $A$. Here $\epsilon$ is left adjoint to $\gamma$, and $\lambda$ is the left $A$-module action on $A \vee J$.

So far we did not use that $M=C X$ is free. Now we use this, and the proof of Lemma 10.9 , to see that the derivation $d$ is corepresented by the composite map

$$
\psi^{\prime}: S[X] \stackrel{i}{\rightarrow} S[M] \stackrel{d}{\rightarrow} A \vee J \stackrel{p}{\rightarrow} J
$$

of symmetric spectra. The factorization of $d$ gives the following factorization

$$
S[X] \stackrel{i}{\rightarrow} S[M] \stackrel{S[\Delta]}{\longrightarrow} S[M \times M] \simeq S[M] \wedge S[M] \stackrel{\bar{\alpha} \wedge \epsilon}{\longrightarrow} A \wedge B^{\infty} M
$$

of $\psi^{\prime}$. We can rewrite this as

$$
S[X] \stackrel{S[\Delta]}{\longrightarrow} S[X \times X] \simeq S[X] \wedge S[X] \stackrel{\bar{\alpha}_{X} \wedge \text { id }}{\longrightarrow} A \wedge S[X] \simeq A \wedge B^{\infty} M,
$$

by noting that the composite

$$
S[X] \stackrel{i}{\rightarrow} S[M] \stackrel{\epsilon}{\rightarrow} B^{\infty} M
$$

is the weak equivalence of Lemma 6.22. The map $\psi$ is the $A$-module extension of $\psi^{\prime}$, hence is given by the composite

$$
\begin{aligned}
A \wedge S[X] \stackrel{\mathrm{id} \wedge S[\Delta]}{\longrightarrow} A \wedge S[X \times X] & \simeq A \wedge S[X] \wedge S[X] \\
& \stackrel{\mathrm{id} \wedge \bar{\alpha}_{X} \wedge \mathrm{id}}{\longrightarrow} A \wedge A \wedge S[X] \stackrel{\mu \wedge \mathrm{id}}{\longrightarrow} A \wedge S[X] .
\end{aligned}
$$

Remark 11.18 For a map $(R, P) \rightarrow(A, M)$ of pre-log symmetric ring spectra, such that $(A, R)$ is a $\mathrm{CW}$ pair in commutative symmetric ring spectra and $(M, P)$ is a $\mathrm{CW}$ pair in commutative $\mathcal{I}$-space monoids, we can determine $A \wedge \wedge_{S[M]} \operatorname{TAQ}^{S[P]}(S[M])$, $A \wedge\left(B^{\infty} M / B^{\infty} P\right)$ and $\psi$ modulo the skeleton filtration, as in Remark 10.10.

Definition 11.19 Let $\left(e, e^{b}\right):(R, P, \rho) \rightarrow(A, M, \alpha)$ be a map of pre-log symmetric ring spectra. The log topological André-Quillen homology of $(A, M)$ over $(R, P)$, denoted TAQ ${ }^{(R, P)}(A, M)$ or TAQ $((A, M) /(R, P))$, is defined by the homotopy pushout 
square

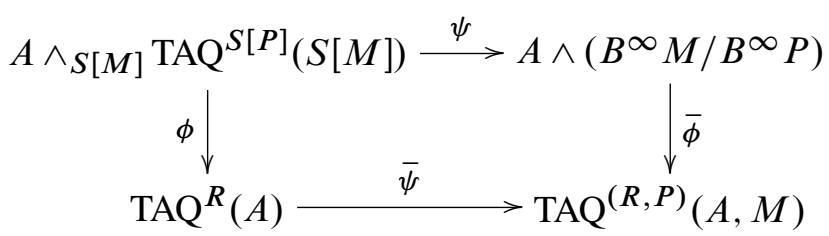

of $A$-modules. Here $\phi$ is induced by $\left(e, e^{b}\right)$ and corepresents $\phi^{*}$ as in Lemma 11.15, while $\psi$ corepresents $\psi^{*}$ as in Lemma 11.16 . We say that $\left(e, e^{b}\right)$ is formally log étale if $\mathrm{TAQ}^{(R, P)}(A, M)$ is contractible. When $(R, P)=(S, 1)$ we simply write $\operatorname{TAQ}(A, M)$ for $\mathrm{TAQ}^{(R, P)}(A, M)$.

Remark 11.20 By analogy with the notation in Definition 4.25, we think of

$$
\bar{\phi}: A \wedge\left(B^{\infty} M / B^{\infty} P\right) \rightarrow \operatorname{TAQ}^{(R, P)}(A, M)
$$

as generating the log differentials, symbolically taking $a \wedge \gamma(m)$ to $a d \log m$. We think of $\bar{\psi}: \mathrm{TAQ}^{R}(A) \rightarrow \mathrm{TAQ}^{(R, P)}(A, M)$ as the inclusion of the ordinary differentials among the log differentials. The pushout along $A \wedge S[M] \mathrm{TAQ}^{S[P]}(S[M])$ imposes the symbolic relations $d \alpha(m)=\alpha(m) d \log m$ between these differentials.

Proposition 11.21 The log topological André-Quillen homology corepresents log derivations, in the sense that there is a natural weak equivalence

$$
\mathcal{M}_{A}\left(\mathrm{TAQ}^{(R, P)}(A, M), J\right) \simeq \operatorname{Der}_{(R, P)}((A, M), J)
$$

of mapping spaces. There is a universal log derivation

$$
\left(d_{u}, d_{u}^{\mathrm{b}}\right):(A, M) \rightarrow\left(A \vee \mathrm{TAQ}^{(R, P)}(A, M), \eta^{*} M\right)
$$

of $(A, M)$ over $(R, P)$ that corresponds to the identity map of $\operatorname{TAQ}^{(R, P)}(A, M)$.

Proof This is clear from Proposition 11.13 and Lemmas 11.15 and 11.16.

Corollary 11.22 A map $\left(e, e^{b}\right):(R, P, \rho) \rightarrow(A, M, \alpha)$ of pre-log symmetric ring spectra is formally log étale if and only if all spaces of log derivations of $(A, M)$ over $(R, P)$ are contractible, ie, if the space of dashed arrows in diagram (11-1) is contractible for each $A$-module $J$.

Corollary 11.23 The logification maps

$$
(R, P) \rightarrow\left(R, P^{a}\right) \quad \text { and } \quad(A, M) \rightarrow\left(A, M^{a}\right)
$$


induce weak equivalences

$$
\mathrm{TAQ}^{(R, P)}(A, M) \stackrel{\simeq}{\rightarrow} \mathrm{TAQ}^{(R, P)}\left(A, M^{a}\right) \stackrel{\simeq}{\longrightarrow} \mathrm{TAQ}^{\left(R, P^{a}\right)}\left(A, M^{a}\right) .
$$

Hence the log topological André-Quillen homology is insensitive to logification.

In particular, $\bar{\psi}: \mathrm{TAQ}^{R}(A) \rightarrow \mathrm{TAQ}^{(R, P)}(A, M)$ is a weak equivalence for each strict $\log$ map $\left(e, e^{b}\right):(R, P) \rightarrow(A, M)$, so a strict map of log symmetric ring spectra is formally log étale if and only if the underlying map of commutative symmetric ring spectra is formally étale.

Proof The first claims are clear from Proposition 11.21 and Lemma 11.9. The second claims follow, since for a strict map $M \simeq e^{*} P=P^{a}$, and it is clear from Definition 11.19 that $\mathrm{TAQ}^{R}(A) \simeq \mathrm{TAQ}^{(R, P)}(A, P)$.

For an alternative proof, starting with the free case $M=C X$, note that by Proposition 11.17 the map $\psi$ is an equivalence when $\alpha: M \rightarrow \Omega_{\otimes}^{\bullet} A$ factors through $\mathrm{GL}_{1}(A)$. A homotopy inverse can be constructed by replacing $\bar{\alpha}_{X}: S[X] \rightarrow A$ with a multiplicative inverse. Hence $\bar{\psi}: \operatorname{TAQ}(A) \rightarrow \operatorname{TAQ}(A, M)$ is a weak equivalence when $(M, \alpha)^{a}$ is trivial. The general case follows by $\mathrm{CW}$ approximation and induction, using the transitivity and flat base change results of Propositions 11.28 and 11.29.

Remark 11.24 When $A=S[M]$, both maps

$$
\begin{aligned}
& \phi: A \wedge S[M] \operatorname{TAQ}(S[M]) \rightarrow \operatorname{TAQ}(A) \\
& \bar{\phi}: A \wedge B^{\infty} M \rightarrow \operatorname{TAQ}(S[M], M)
\end{aligned}
$$

are weak equivalences, so the comparison map

$$
\bar{\psi}: \operatorname{TAQ}(S[M]) \rightarrow \operatorname{TAQ}(S[M], M)
$$

is identified with the map $\psi$ that was described in Proposition 11.17 for free $M$.

Lemma 11.25 Let $\left(e, e^{b}\right):(R, P) \rightarrow(A, M)$ be a map of pre-log symmetric ring spectra, and let $C=R \wedge_{S[P]} S[M]$, so that the left hand square is a pushout in the following diagram

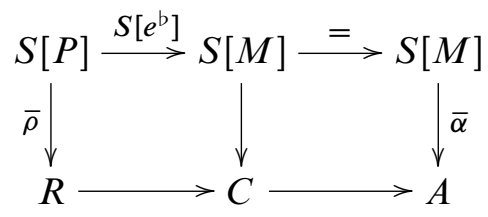


of commutative symmetric ring spectra. Then there is a natural homotopy cofiber sequence

$$
A \wedge\left(B^{\infty} M / B^{\infty} P\right) \stackrel{\bar{\phi}}{\rightarrow} \mathrm{TAQ}^{(R, P)}(A, M) \rightarrow \mathrm{TAQ}^{C}(A)
$$

of $A$-modules. Hence $(R, P) \rightarrow(A, M)$ is formally log étale if and only if the connecting map

$$
\text { д: } \operatorname{TAQ}^{C}(A) \rightarrow \Sigma A \wedge\left(B^{\infty} M / B^{\infty} P\right)
$$

is an equivalence.

Proof By the homotopy pushout square of Definition 11.19, the homotopy cofiber of

$$
\phi: A \wedge S[M] \mathrm{TAQ}^{S[P]}(S[M]) \rightarrow \mathrm{TAQ}^{R}(A)
$$

is equivalent to the homotopy cofiber of

$$
\bar{\phi}: A \wedge\left(B^{\infty} M / B^{\infty} P\right) \rightarrow \mathrm{TAQ}^{(R, P)}(A, M) .
$$

By flat base change along $\bar{\rho}: S[P] \rightarrow R$ we have an equivalence

$$
C \wedge S[M] \operatorname{TAQ}^{S[P]}(S[M]) \simeq \operatorname{TAQ}^{R}(C)
$$

so we can rewrite $\phi$ as the map

$$
A \wedge_{C} \mathrm{TAQ}^{R}(C) \rightarrow \mathrm{TAQ}^{R}(A)
$$

with homotopy cofiber $\mathrm{TAQ}^{C}(A)$, by the transitivity sequence for $R \rightarrow C \rightarrow A$.

Remark 11.26 It is clear that $(R, P) \rightarrow(A, M)$ will be formally log étale if $B^{\infty} P \rightarrow$ $B^{\infty} M$ is an $A$-homology equivalence (so $A \wedge\left(B^{\infty} M / B^{\infty} P\right) \simeq *$ ) and $C \rightarrow A$ is formally étale (so $\operatorname{TAQ}^{C}(A) \simeq *$ ). The converse holds in the algebraic context of fine log schemes (or fine log rings), as proved by Kato [35, 3.5]. In the topological context it remains to be determined whether $\partial$ can be an equivalence, in cases where $\mathrm{TAQ}^{C}(A)$ and $A \wedge\left(B^{\infty} M / B^{\infty} P\right)$ are not trivial.

Lemma 11.27 Let $(A, M, \alpha)$ be a pre-log symmetric ring spectrum. The map

$$
\bar{\psi}: \operatorname{TAQ}(A) \rightarrow \operatorname{TAQ}(A, M)
$$

is a weak equivalence if and only if the logification $(M, \alpha)^{a}$ is equivalent to the trivial $\log$ structure. 
Proof The map $\bar{\psi}$ is a weak equivalence if and only if the map $\psi^{*}$ of Proposition 11.13 is a weak equivalence, for all $A$-modules $J$. Here $\psi^{*}$ takes a section $d^{\mathrm{b}}: M \rightarrow \eta^{*} M$ to its composite with $\eta^{*} \alpha$, shown as dashed arrows in the diagram

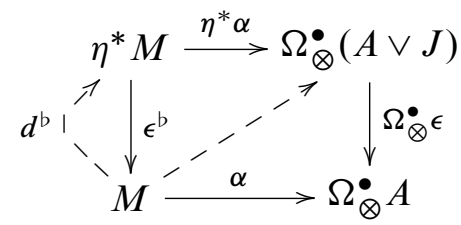

of commutative $\mathcal{I}$-space monoids. Hence $\psi^{*}$ is a weak equivalence if and only if the solid square is homotopy cartesian. Now $\eta^{*} M \simeq M \times\left(1+\Omega^{\bullet} J\right)_{\otimes} \simeq M \times \Omega^{\bullet} J$ by Lemma 11.2, and $\Omega_{\otimes}^{\bullet}(A \vee J) \simeq \Omega_{\otimes}^{\bullet} A \times \Omega^{\bullet} J$. By Lemma 11.5, $\eta^{*} \alpha$ maps the homotopy fiber $\Omega^{\bullet} J$ over $m$ to the homotopy fiber $\Omega^{\bullet} J$ over $\alpha(m)$ by multiplication by $\alpha(m)$. Hence this is an equivalence, for all $A$-modules $J$, if and only if $\alpha(m)$ is homotopy invertible for all $m$. In other words, this holds precisely when $\alpha: M \rightarrow \Omega_{\otimes}^{\bullet} A$ has image contained in $\mathrm{GL}_{1}(A)$, which is equivalent to the condition that $(M, \alpha)^{a}$ agrees with the trivial log structure on $A$.

Proposition 11.28 Let $(R, P) \stackrel{\left(e, e^{\mathrm{b}}\right)}{\longrightarrow}(A, M) \stackrel{\left(f, f^{\mathrm{b}}\right)}{\longrightarrow}(B, N)$ be maps of pre-log symmetric ring spectra. There is a natural homotopy cofiber sequence

$$
B \wedge_{A} \operatorname{TAQ}^{(R, P)}(A, M) \rightarrow \mathrm{TAQ}^{(R, P)}(B, N) \rightarrow \mathrm{TAQ}^{(A, M)}(B, N)
$$

of $B$-modules, known as the transitivity sequence for $\left(e, e^{b}\right)$ and $\left(f, f^{b}\right)$.

Proof The sequence corepresents a homotopy fiber sequence

$$
\operatorname{Der}_{(A, M)}((B, N), K) \rightarrow \operatorname{Der}_{(R, P)}((B, N), K) \rightarrow \operatorname{Der}_{(R, P)}\left((A, M), f^{\#} K\right),
$$

for all $B$-modules $K$, hence is a homotopy cofiber sequence. For a different argument, consider the commutative diagram:

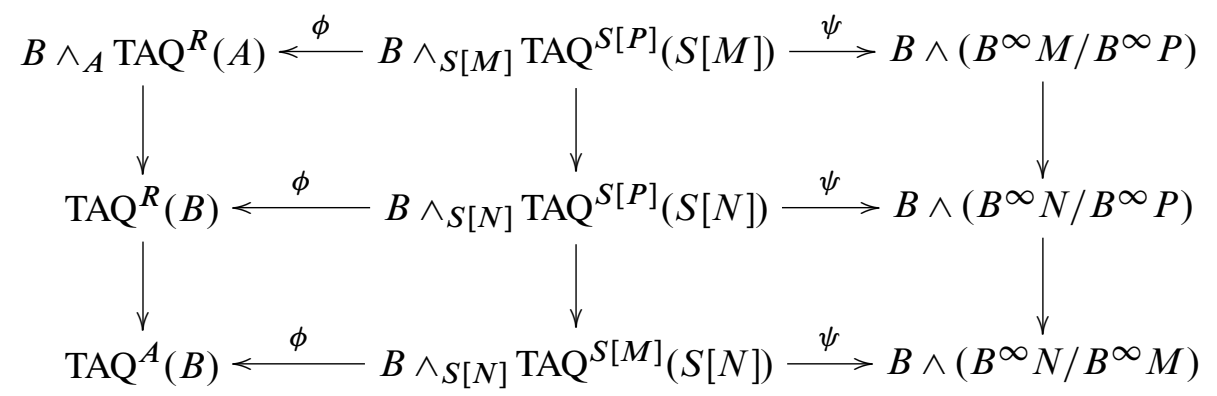


The left hand and middle columns are homotopy cofiber sequences by Proposition 10.7, and this is clear for the right hand column. Hence the column of homotopy pushouts is also a homotopy cofiber sequence.

Proposition 11.29 Let

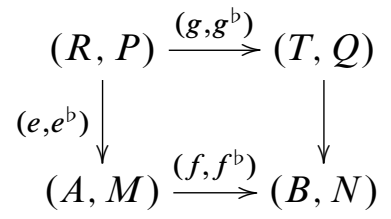

be a pushout square of pre-log symmetric ring spectra, so $B=T \wedge_{R} A$ and $N=$ $Q \oplus_{P} M$. There is a natural weak equivalence

$$
\begin{aligned}
B \wedge_{A} \operatorname{TAQ}^{(R, P)}(A, M)=T & \wedge \operatorname{TAQ}^{(R, P)}(A, M) \\
& \stackrel{\simeq}{\rightarrow} \operatorname{TAQ}^{(T, Q)}\left(T \wedge_{R} A, Q \oplus_{P} M\right)=\operatorname{TAQ}^{(T, Q)}(B, N)
\end{aligned}
$$

known as flat base change along $\left(g, g^{b}\right)$.

Proof By the pushout property of the left hand square below

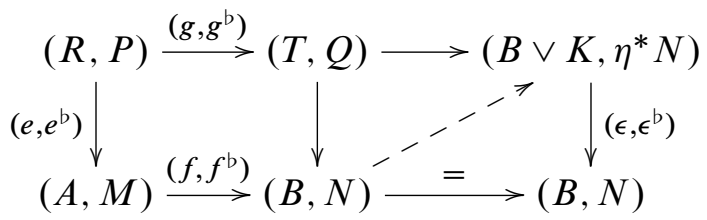

where $K$ is a $B$-module, the space of dashed lifts is equivalent to the space

$$
(\operatorname{Pre} \log (R, P) /(B, N))\left((A, M),\left(B \vee K, \eta^{*} N\right)\right)
$$

of lifts across the outer rectangle, which is weakly equivalent to

$$
\operatorname{Der}_{(R, P)}\left((A, M), f^{\#} K\right)
$$

by the log analogue of Lemma 10.6, since

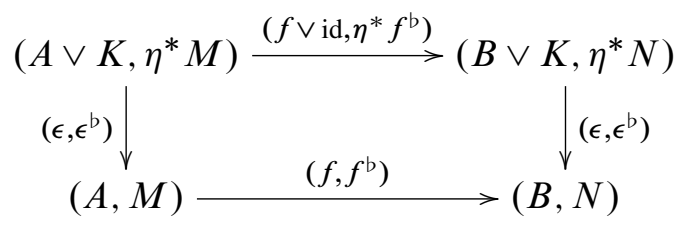

is a homotopy pullback square. Hence $\operatorname{Der}_{(T, Q)}((B, N), K)$ is corepresented by

$$
B \wedge_{A} \operatorname{TAQ}^{(R, P)}(A, M) \cong T \wedge_{R} \operatorname{TAQ}^{(R, P)}(A, M) .
$$


For an alternative proof, note that there is a pushout square

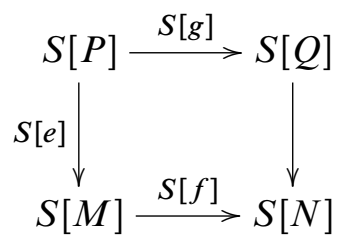

of commutative symmetric ring spectra, and a homotopy pushout square

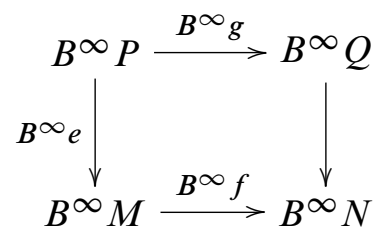

of symmetric spectra. Hence the vertical maps in the following diagram

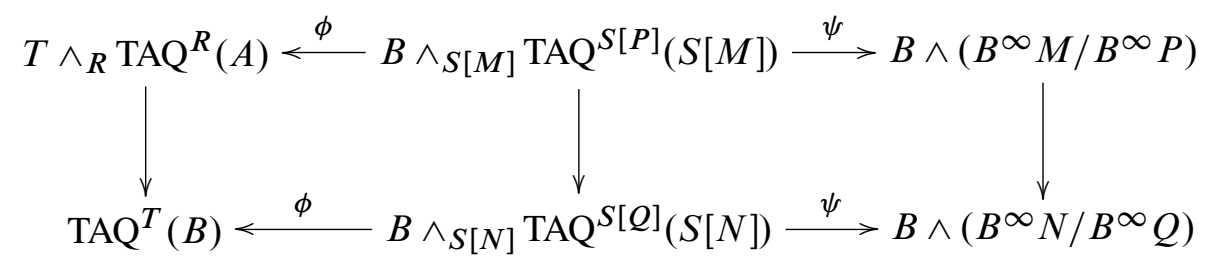

are weak equivalences, by Proposition 10.8. Hence the induced map of homotopy pushouts is also a weak equivalence. Replacing $B$ by $T \wedge_{R} A$ in the upper row, we obtain the flat base change equivalence.

Proposition 11.30 Let $(A, M) \rightarrow(R, P)$ be a virtually surjective map of pre-log symmetric ring spectra, in the sense that $\left(\pi_{0} M\right)^{\mathrm{gp}}=\pi_{0} \Gamma M \rightarrow \pi_{0} \Gamma P=\left(\pi_{0} P\right)^{\mathrm{gp}}$ is surjective. Let $M^{\text {rep }}=P \times_{\Gamma P} \Gamma M$ be the repletion of $M$ over $P$, and let $A^{\text {rep }}=$ $A \wedge_{S[M]} S\left[M^{\text {rep }}\right]$. Then there is a weak equivalence

$$
A^{\mathrm{rep}} \wedge_{A} \operatorname{TAQ}(A, M) \stackrel{\simeq}{\rightarrow} \operatorname{TAQ}\left(A^{\mathrm{rep}}, M^{\mathrm{rep}}\right),
$$

and the repletion map $(A, M) \rightarrow\left(A^{\text {rep }}, M^{\text {rep }}\right)$ is formally log étale. In this sense, $\log$ topological André-Quillen homology commutes with repletion. 
Proof Consider the following diagram

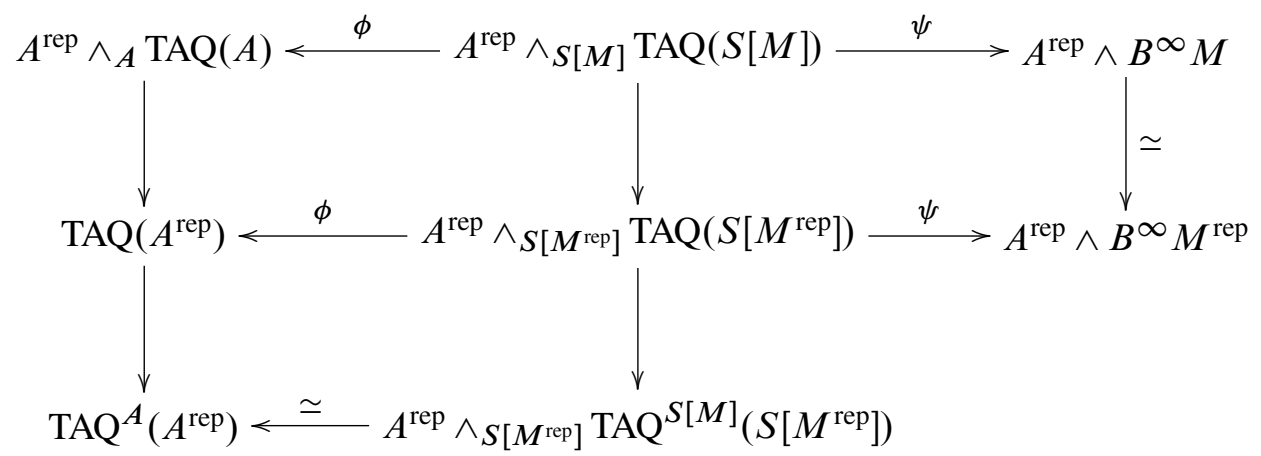

of $A^{\text {rep }}$-modules. The left hand and middle columns are homotopy cofiber sequences by transitivity (Proposition 11.28). The right hand vertical map is an equivalence by Proposition 8.3, since $\Gamma M \rightarrow \Gamma\left(M^{\text {rep }}\right)$ is an equivalence. The bottom horizontal map is an equivalence by flat base change along $S[M] \rightarrow A$. Hence the induced map of horizontal homotopy pushouts

$$
A^{\mathrm{rep}} \wedge_{A} \mathrm{TAQ}(A, M) \stackrel{\simeq}{\longrightarrow} \mathrm{TAQ}\left(A^{\mathrm{rep}}, M^{\text {rep }}\right)
$$

is a weak equivalence. By transitivity, $\mathrm{TAQ}^{(A, M)}\left(A^{\text {rep }}, M^{\text {rep }}\right)$ is contractible.

Corollary 11.31 Let $\left(R_{0}, P_{0}\right)$ be a base pre-log symmetric ring spectrum and let

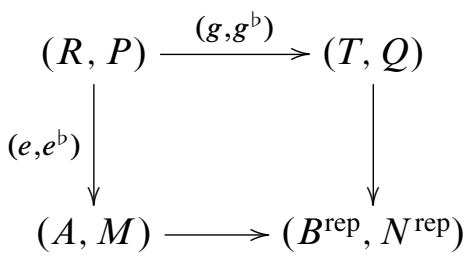

be a pushout square of replete pre-log symmetric ring spectra over $\left(R_{0}, P_{0}\right)$, so that $N^{\text {rep }}=Q \oplus_{P}^{\text {rep }} M$ is the repletion of $N=Q \oplus_{P} M$ over $P_{0}, B=T \wedge_{R} A$ and $B^{\text {rep }}=B \wedge S[N] S\left[N^{\text {rep }}\right]$. In other words, $\left(B^{\text {rep }}, N^{\text {rep }}\right)=(T, Q) \wedge_{(R, P)}^{\text {rep }}(A, M)$. Then there is a natural weak equivalence

$$
B^{\text {rep }} \wedge_{A} \operatorname{TAQ}^{(R, P)}(A, M) \stackrel{\simeq}{\rightarrow} \mathrm{TAQ}^{(T, Q)}\left(B^{\text {rep }}, N^{\text {rep }}\right)
$$

of $B^{\text {rep }}$-modules, which we call replete base change along $\left(g, g^{b}\right)$. 


\section{Based logarithmic topological André-Quillen homology}

We now turn to based log derivations.

Definition 12.1 Let $(N, \alpha)$ be a based log structure on a commutative symmetric ring spectrum $A$, and let $J$ be an $A$-module. The inverse image based log structure $\left(\eta^{*} N, \eta^{*} \alpha\right)$ of $(N, \alpha)$ along $\eta: A \rightarrow A \vee J$ is given by the upper central pushout square in the diagram

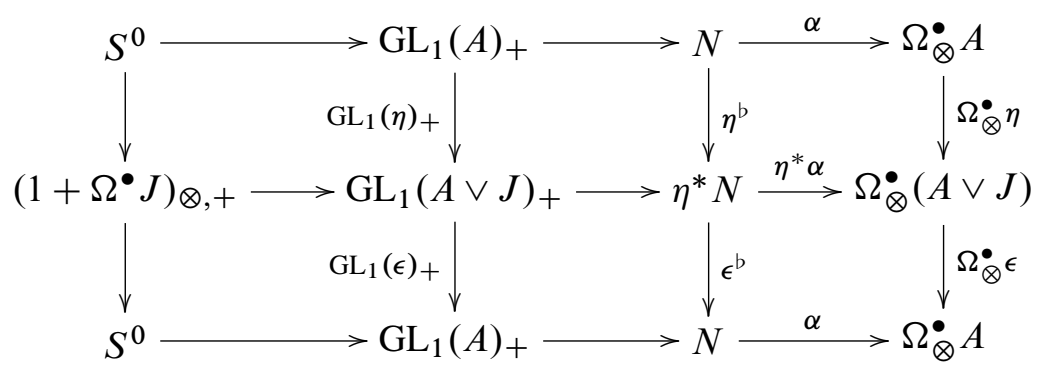

of commutative based $\mathcal{I}$-space monoids. The upper left hand square is a homotopy pushout, so we get weak equivalences

$$
\begin{aligned}
\eta^{*} N & \simeq N \boxminus\left(1+\Omega^{\bullet} J\right)_{\otimes,+} \\
& \simeq N \wedge\left(1+\Omega^{\bullet} J\right)_{\otimes,+} \simeq N \wedge\left(\Omega^{\bullet} J\right)_{+} .
\end{aligned}
$$

When $(N, \alpha)$ is only a based pre-log structure, we define $\eta^{*} N$ by these formulas.

Definition 12.2 Let $N$ be a commutative based $\mathcal{I}$-space monoid and let $K$ be a grouplike $\mathcal{I}$-space monoid. The space of commutative based $\mathcal{I}$-space monoid derivations of $N$ with values in $K$ is the space $\operatorname{Der}_{0}^{\mathrm{b}}(N, K)$ of dashed arrows $d^{\mathrm{b}}$ making the diagram

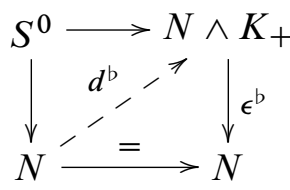

of commutative based $\mathcal{I}$-space monoids commute.

The space $\operatorname{Der}_{0}((A, N), J)$ of based log derivations of a based pre-log symmetric ring spectrum $(A, N)$ with values in $J$ is defined similarly, consisting of pairs $\left(d, d^{\mathrm{b}}\right)$ where $d: A \rightarrow A \vee J$ is a derivation and $d^{b}: N \rightarrow \eta^{*} N \simeq N \wedge\left(\Omega^{\bullet} J\right)_{+}$is a commutative 
based $\mathcal{I}$-space monoid derivation, such that the diagram

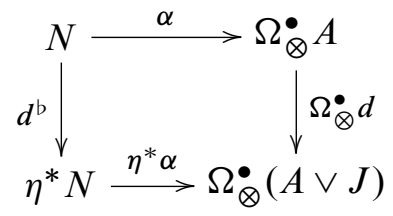

commutes.

Lemma 12.3 Let $Y=\operatorname{cone}(L) \cup_{L} Y^{\prime}$ be a conically based $\mathcal{I}$-space, and let $N=C_{0} Y$. There are natural equivalences

$$
\operatorname{Der}_{0}^{\mathrm{b}}(N, K) \simeq \mathcal{S}^{\mathcal{I}}\left(Y^{\prime}, K\right) \simeq \mathcal{S} p^{\Sigma}\left(S\left[Y^{\prime}\right], B^{\infty} K\right)
$$

for all grouplike commutative $\mathcal{I}$-space monoids $K$.

Proof Since $N=C_{0} Y$ is free, the commutative based $\mathcal{I}$-space monoid derivations $d^{\text {b }}: N \rightarrow N \wedge K_{+}$are equivalent to the based $\mathcal{I}$-space maps $Y \rightarrow\left(C_{0} Y\right) \wedge K_{+}$over $C_{0} Y$, or equivalently, to the based $\mathcal{I}$-space maps $f: Y \rightarrow Y \wedge K_{+}$over $Y$. We think of such maps as graphs of maps $Y \rightarrow K$, except that special care is required near the base point 0 of $Y$. Using the cone coordinate in cone $(L) \subset Y$, any such map $f$ can deformed to a map $g$ that is constant in the cone direction over cone $(L)$. The deformation collapses a growing neighborhood of the cone vertex to that vertex, while stretching a complementary neighborhood of the base to cover the cone. This way, the graph over cone $(L)$ flows into the special fiber $0 \times K \subset Y \times K$, gradually becoming independent of the cone coordinate. The deformation is constant over $Y^{\prime}$. The end map $g$ is simplicial/continuous at the base point of $Y$ because the special fiber has been collapsed in $Y \wedge K_{+}$. By restriction over $Y^{\prime} \subset Y$ we get an equivalence between these maps $g$ and the space of $\mathcal{I}$-space maps $Y^{\prime} \rightarrow Y^{\prime} \times K$ over $Y^{\prime}$, which we identify with the space of $\mathcal{I}$-space maps $Y^{\prime} \rightarrow K$.

This deformation retraction provides the first natural equivalence. The second equivalence is standard, since $K \simeq \Omega^{\bullet} B^{\bullet} K$ for grouplike $K$.

Lemma 12.4 Let $N=\operatorname{cone}(L) \cup_{L} N^{\prime}$ be a commutative conically based $\mathcal{I}$-space monoid. There are natural equivalences

$$
\operatorname{Der}_{0}^{\mathrm{b}}(N, K) \simeq \mathcal{C S}^{\mathcal{I}}\left(N^{\prime}, K\right) \simeq \mathcal{S} p^{\Sigma}\left(B^{\infty}\left(N^{\prime}\right), B^{\infty} K\right)
$$

for all grouplike commutative $\mathcal{I}$-space monoids $K$. 
Proof By a deformation retraction like that in the proof of Lemma 12.3, the space $\operatorname{Der}_{0}^{\mathrm{b}}(N, K)$ is equivalent to the space of commutative $\mathcal{I}$-space monoid maps $N \rightarrow$ $N \wedge K_{+}$over $N$ that are constant in the cone direction over cone $(L) \subset N$. This space is identified with the space of commutative $\mathcal{I}$-space monoid maps $N^{\prime} \rightarrow K$, and is equivalent to the space of symmetric spectrum maps $B^{\infty}\left(N^{\prime}\right) \rightarrow B^{\infty} K$, since $K$ is grouplike.

Proposition 12.5 Let $(A, N)$ be a based pre-log symmetric ring spectrum, and $J$ an $A$-module. There is a homotopy pullback square

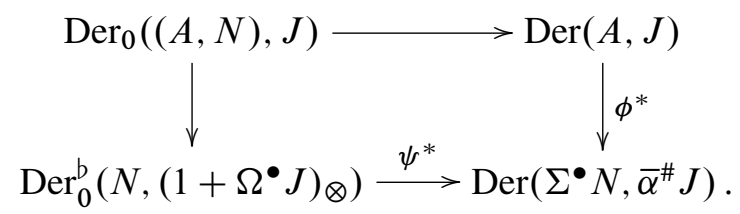

Here $\phi^{*}$ is corepresented by the map $\phi: A \wedge^{\bullet}{ }^{*} \operatorname{TAQ}\left(\Sigma^{\bullet} N\right) \rightarrow \operatorname{TAQ}(A)$ induced by $\bar{\alpha}: \Sigma^{\bullet} N \rightarrow A$, and $\psi^{*}$ takes a commutative based $\mathcal{I}$-space derivation $d^{\mathrm{b}}: N \rightarrow$ $N \wedge K_{+}$to the composite $\eta^{*} \alpha \circ d^{\mathrm{b}}$ with $\eta^{*} \alpha: N \wedge K_{+} \rightarrow \Omega_{\otimes}^{\bullet}(A \vee J)$, interpreted in adjoint form as a commutative symmetric ring spectrum map $\Sigma^{\bullet} N \rightarrow A \vee J$ over $A$, or equivalently, as a derivation of $\Sigma^{\bullet} N$ with values in $\bar{\alpha}^{\#} J$.

Definition 12.6 Let $(A, N, \alpha)$ be a based pre-log symmetric ring spectrum, with $N=\operatorname{cone}(L) \cup_{L} N^{\prime}$ conically based. We define the based log topological AndréQuillen homology of $(A, N)$, denoted $\mathrm{TAQ}_{0}(A, N)$, by the pushout square

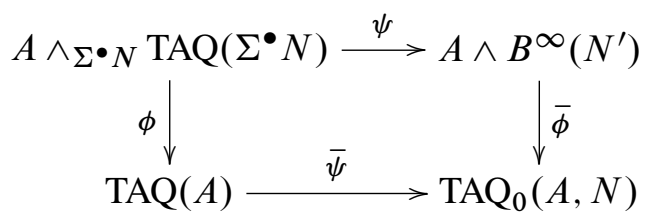

of $A$-modules. Here $\phi$ is induced by $\bar{\alpha}$, and $\psi$ corepresents the natural map $\psi^{*}$ described in Proposition 12.5.

More generally, for a map $\left(e, e^{b}\right):(R, Q, \rho) \rightarrow(A, N, \alpha)$ of conically based pre-log symmetric ring spectra, define $\operatorname{TAQ}_{0}^{(R, Q)}(A, N)$ by the pushout square

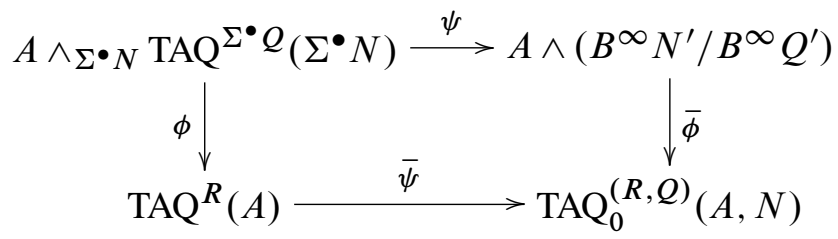


of $A$-modules. When $\mathrm{TAQ}_{0}^{(R, Q)}(A, N) \simeq *$, we say that $(R, Q) \rightarrow(A, N)$ is formally based log étale.

Proposition 12.7 Let $(A, N, \alpha)$ be a based pre-log symmetric ring spectrum, and assume that $N=C_{0} Y$ is the free commutative based $\mathcal{I}$-space monoid on a conically based $\mathcal{I}$-space $Y=\operatorname{cone}(L) \cup_{L} Y^{\prime}$. Let $\bar{\alpha}_{Y}=\bar{\alpha} \circ i$ be the composite

$$
\Sigma^{\bullet} Y \stackrel{i}{\rightarrow} \Sigma^{\bullet} N \stackrel{\bar{\alpha}}{\rightarrow} A
$$

The corepresenting map

$$
\psi: A \wedge_{\Sigma}{ }_{N} \operatorname{TAQ}\left(\Sigma^{\bullet} N\right) \rightarrow A \wedge B^{\infty}\left(N^{\prime}\right)
$$

factors as

$$
\begin{aligned}
A \wedge \Sigma^{\bullet} N & \operatorname{TAQ}\left(\Sigma^{\bullet} N\right) \\
& \simeq A \wedge \Sigma^{\bullet} Y \\
& \stackrel{\text { id } \wedge \Sigma^{\bullet} \delta}{\longrightarrow} A \wedge \Sigma^{\bullet}\left(Y \wedge Y_{+}^{\prime}\right) \simeq A \wedge \Sigma^{\bullet} Y \wedge S\left[Y^{\prime}\right] \\
& \stackrel{\text { id } \wedge \bar{\alpha}_{Y} \wedge \text { id }}{\longrightarrow} A \wedge A \wedge S\left[Y^{\prime}\right] \\
& \stackrel{\mu \wedge \text { id }}{\longrightarrow} A \wedge S\left[Y^{\prime}\right] \simeq A \wedge B^{\infty}\left(N^{\prime}\right)
\end{aligned}
$$

where the conical diagonal map $\delta: Y \rightarrow Y \wedge Y_{+}^{\prime}$ restricts to the diagonal over $Y^{\prime}$, and is constant in the cone direction over cone $(L) \subset Y$.

Proof The proof is similar to that of Proposition 11.17. Let $J=A \wedge B^{\infty}\left(N^{\prime}\right)$. The commutative based $\mathcal{I}$-space derivation $d^{\mathrm{b}}: N \rightarrow \eta^{*} N=N \boxminus \Omega^{\bullet} J_{+} \simeq N \wedge \Omega^{\bullet} J_{+}$ factors as $\delta: N \rightarrow N \wedge N_{+}^{\prime}$ composed with id $\wedge \gamma_{+}^{\prime}$, where $\gamma^{\prime}: N^{\prime} \rightarrow \Omega^{\bullet}\left(A \wedge B^{\bullet} N^{\prime}\right) \simeq$ $\Omega^{\bullet} J$. Hence the composite

$$
N \stackrel{d^{b}}{\rightarrow} N \wedge \Omega^{\bullet} J_{+} \stackrel{\eta^{*} \alpha}{\longrightarrow} \Omega^{\infty}(A \vee J) \stackrel{p}{\rightarrow} \Omega^{\infty} J
$$

is right adjoint to the composite

$$
\Sigma^{\bullet} N \stackrel{\Sigma^{\bullet} \delta}{\longrightarrow} \Sigma^{\bullet} N \wedge S\left[N^{\prime}\right] \stackrel{\bar{\alpha} \wedge \epsilon_{+}}{\longrightarrow} A \wedge B^{\infty}\left(N^{\prime}\right) .
$$

Using that $N=C_{0} Y$ is free, we find (as in Lemma 10.9) that $\eta^{*} \alpha \circ d^{\mathrm{b}}$ is right adjoint to the derivation $d$ of $\Sigma^{\bullet} N$ that is corepresented by the composite map

$$
\psi^{\prime}: \Sigma^{\bullet} Y \stackrel{\Sigma^{\bullet} \delta}{\longrightarrow} \Sigma^{\bullet} Y \wedge S\left[Y^{\prime}\right] \stackrel{\bar{\alpha}_{Y} \wedge \text { id }}{\longrightarrow} A \wedge S\left[Y^{\prime}\right]
$$

of symmetric spectra. The equivalence $B^{\infty}\left(N^{\prime}\right) \simeq S\left[Y^{\prime}\right]$ is from Lemma 6.22. The map $\psi$ is the $A$-module extension of $\psi^{\prime}$, giving the claimed factorization. 
Lemma 12.8 If $N=M_{+}$has a disjoint zero, then $\operatorname{TAQ}_{0}(A, N) \cong \operatorname{TAQ}(A, M)$.

Proof This is clear from $N^{\prime}=M$ and Definitions 11.19 and 12.6.

Example 12.9 Let $Y=\operatorname{cone}(L) \cup_{L} Y^{\prime}$ be a conically based $\mathcal{I}$-space. It can be expressed as a pushout

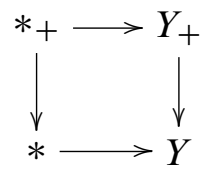

in the category of based $\mathcal{I}$-spaces, where $*_{+}=S^{0}, Y_{+}$and $*=\{0\}$ all have disjoint zeros. However, this is usually not a pushout of conically based $\mathcal{I}$-space. Applying $C_{0}$ we get a pushout square

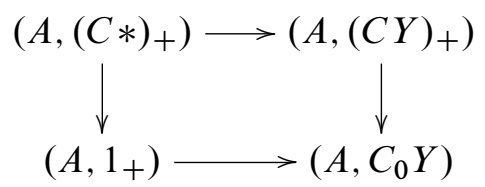

of based pre-log symmetric ring spectra, for any pre-log structure $\alpha: C_{0} Y \rightarrow \Omega_{\otimes}^{\bullet} A$. There is no base change formula for based $\log$ TAQ in this case, since the square of symmetric spectra

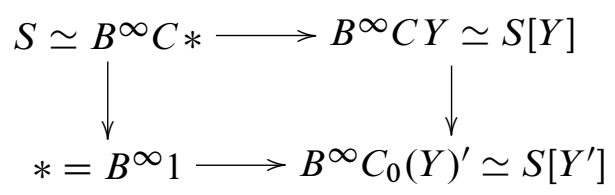

can only be a homotopy pushout if $\Sigma^{\infty}(\operatorname{cone}(L) / L) \simeq S$, which mostly happens for $L=\varnothing$. Here we have used Lemma 6.22 in every corner. On the other hand there is a base change formula for pushouts of conically based pre-log structures.

Proposition 12.10 Let $(R, P) \stackrel{\left(e, e^{\mathrm{b}}\right)}{\longrightarrow}(A, M) \stackrel{\left(f, f^{\mathrm{b}}\right)}{\longrightarrow}(B, N)$ be maps of conically based pre-log symmetric ring spectra. There is a natural homotopy cofiber sequence

$$
B \wedge_{A} \operatorname{TAQ}_{0}^{(R, P)}(A, M) \rightarrow \mathrm{TAQ}_{0}^{(R, P)}(B, N) \rightarrow \mathrm{TAQ}_{0}^{(A, M)}(B, N)
$$

of $B$-modules, known as the based transitivity sequence.

Proof The proof is practically identical to that of Proposition 11.28, using the homotopy cofiber sequence

$$
\left(B^{\infty} M^{\prime} / B^{\infty} P^{\prime}\right) \rightarrow\left(B^{\infty} N^{\prime} / B^{\infty} P^{\prime}\right) \rightarrow\left(B^{\infty} N^{\prime} / B^{\infty} M^{\prime}\right)
$$

of symmetric spectra. 


\section{Proposition 12.11 Let}

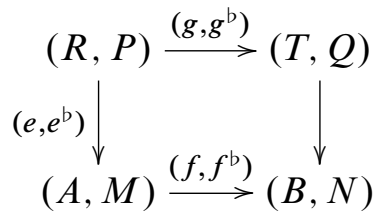

be a pushout square in the category of conically based pre-log symmetric ring spectra. There is a natural weak equivalence

$$
T \wedge_{R} \operatorname{TAQ}_{0}^{(R, P)}(A, M) \stackrel{\simeq}{\rightarrow} \mathrm{TAQ}_{0}^{(T, Q)}(B, N)
$$

known as based flat base change.

Proof The proof is practically identical to that of Proposition 11.29, using the homotopy pushout square

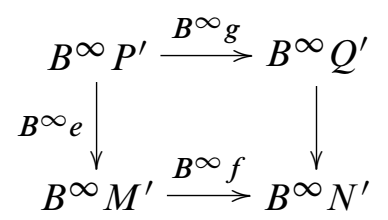

of symmetric spectra.

Example 12.12 Let $B=k u$ be the connective complex $K$-theory spectrum, let $Y \simeq S^{2}$ be a conically based $\mathcal{I}$-space, and let $N=C_{0} Y \simeq C_{0} S^{2}$ be the free commutative conically based $\mathcal{I}$-space monoid generated by $Y$. Let $\beta: N \rightarrow \Omega^{\bullet} k u$ be the commutative based $\mathcal{I}$-space monoid map that extends a based $\mathcal{I}$-space map $u$ : $Y \rightarrow \Omega^{\bullet} k u$ that represents the generator of $\pi_{*}(k u)=\mathbb{Z}[u]$, with $|u|=2$. Then $\operatorname{TAQ}\left(\Sigma^{\bullet} N\right) \simeq \Sigma^{\bullet} N \wedge S^{2}$ by Lemma 10.9. Furthermore, $Y^{\prime} \simeq\left(S^{2}\right)^{\prime} \simeq *, N^{\prime} \simeq$ $C\left(S^{2}\right)^{\prime} \simeq C *$, and $B^{\infty} N^{\prime} \simeq S$, by Lemma 6.22. By Proposition 12.7 the map

$$
\psi: k u \wedge S^{2} \simeq k u \wedge \Sigma^{\bullet} N \operatorname{TAQ}\left(\Sigma^{\bullet} N\right) \rightarrow k u \wedge B^{\infty} N^{\prime} \simeq k u
$$

is the multiplication-by- $u$ map defined as the composite

$$
u \cdot: k u \wedge S^{2} \stackrel{\text { id } \wedge u}{\longrightarrow} k u \wedge k u \stackrel{\mu}{\rightarrow} k u .
$$

This uses that

$$
\delta: S^{2} \rightarrow S^{2} \wedge\left(S^{2}\right)_{+}^{\prime} \simeq S^{2} \wedge S^{0} \cong S^{2}
$$


is homotopic to the identity. Hence we have a homotopy pushout square

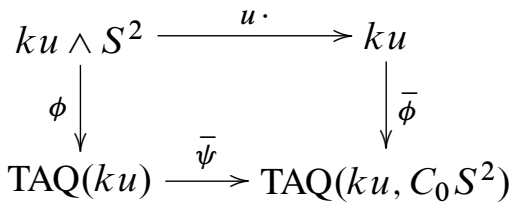

of $k u$-modules.

Example 12.13 Let $A=\ell$ be the $p$-local Adams summand of the connective complex $K$-theory spectrum, let $q=2 p-2$, let $X \simeq S^{q}$ be a conically based $\mathcal{I}$-space, and let $M=C_{0} X \simeq C_{0} S^{q}$ be the free commutative conically based $\mathcal{I}$-space monoid. Let $\alpha: M \rightarrow \Omega \bullet \ell$ be the commutative based $\mathcal{I}$-space monoid map that extends a based $\mathcal{I}$-space map $v_{1}: X \rightarrow \Omega^{\bullet} \ell$ that represents the generator of $\pi_{*}(\ell)=\mathbb{Z}_{(p)}\left[v_{1}\right]$, with $\left|v_{1}\right|=q$. Then $\operatorname{TAQ}\left(\Sigma^{\bullet} M\right) \simeq \Sigma^{\bullet} M \wedge S^{q}$ by Lemma 10.9. Furthermore, $X^{\prime} \simeq\left(S^{q}\right)^{\prime} \simeq *, M^{\prime} \simeq C\left(S^{q}\right)^{\prime} \simeq C *$, and $B^{\infty} M^{\prime} \simeq S$, by Lemma 6.22. By Proposition 12.7 the map

$$
\psi: \ell \wedge S^{q} \simeq \ell \wedge \Sigma^{\bullet} M \operatorname{TAQ}\left(\Sigma^{\bullet} M\right) \rightarrow \ell \wedge B^{\infty} M^{\prime} \simeq \ell
$$

is the multiplication-by- $v_{1}$ map defined as the composite

$$
v_{1}: \ell \wedge S^{q} \stackrel{\text { id } \wedge v_{1}}{\longrightarrow} \ell \wedge \ell \stackrel{\mu}{\rightarrow} \ell .
$$

This uses that

$$
\delta: S^{q} \rightarrow S^{q} \wedge\left(S^{q}\right)_{+}^{\prime} \simeq S^{q} \wedge S^{0} \cong S^{q}
$$

is homotopic to the identity. Hence we have a homotopy pushout square

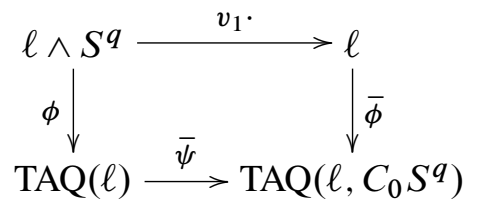

of $\ell$-modules.

Example 12.14 We can compare Example 12.13 and the $p$-local version of Example 12.12 in terms of the based transitivity sequence of Proposition 12.10. We have a map $\left(f, f^{b}\right):(\ell, M) \rightarrow\left(k u_{(p)}, N\right)$ given by the usual inclusion $f: \ell \rightarrow k u_{(p)}$ and the commutative $\mathcal{I}$-space monoid map $f^{b}: M \rightarrow N$, with $M \simeq C_{0} S^{q}$ and $N \simeq C_{0} S^{2}$, that extends the usual map $S^{q} \rightarrow\left(S^{2}\right)^{\square(p-1)} / \Sigma_{p-1} \rightarrow C_{0} S^{2}$. 
Applying base change along $f: \ell \rightarrow k u_{(p)}$ to the map $v_{1} \cdot$, we are led to compare it to the map $u$. via the following commutative square

$$
\begin{aligned}
& k u_{(p)} \wedge S^{q} \stackrel{u^{p-1}}{\longrightarrow} k u_{(p)} \\
&(p-1) u^{p-2} \cdot \simeq \downarrow_{(p-1)} . \\
& k u_{(p)} \wedge S^{2} \stackrel{u \cdot}{\longrightarrow} k u_{(p)}
\end{aligned}
$$

of $k u_{(p)}$-modules. Here $u^{p-1}$ is obtained by base change from $v_{1} \cdot$. The right hand vertical map is induced by the punctured map $f^{b, \prime}: M^{\prime} \rightarrow N^{\prime}$, where $M^{\prime} \simeq C *$ and $N^{\prime} \simeq C *$ and $f^{b, \prime}$ takes the generator of $M^{\prime}$ to the $(p-1)$-th power of the generator of $N^{\prime}$. Hence the map $S \simeq B^{\infty} M^{\prime} \rightarrow B^{\infty} N^{\prime} \simeq S$ corepresenting

$$
\left(f^{\mathrm{b}}\right)^{*}: \operatorname{Der}_{0}^{\mathrm{b}}(N, K) \rightarrow \operatorname{Der}_{0}^{\mathrm{b}}(M, K)
$$

has degree $(p-1)$. Since the square homotopy commutes, the left hand vertical map must be multiplication by the different $(p-1) u^{p-2}$ of $v_{1}=u^{p-1}$. This is compatible with its description as the map of (topologically derived) Kähler differentials

$$
k u_{(p)} \wedge_{\Sigma^{\bullet}} C_{0} S^{q} \operatorname{TAQ}\left(\Sigma^{\bullet} C_{0} S^{q}\right) \rightarrow k u_{(p)} \wedge_{\Sigma^{\bullet}} C_{0} S^{2} \operatorname{TAQ}\left(\Sigma^{\bullet} C_{0} S^{2}\right),
$$

induced by $f^{b}$, taking $d v_{1}=d\left(u^{p-1}\right)$ to $(p-1) u^{p-2} d u$.

We have a similar commutative square

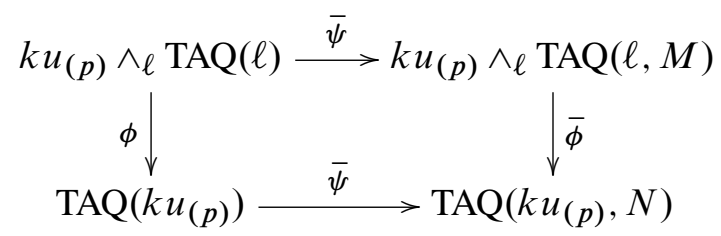

and the vertical homotopy cofibers of the cube formed from these two squares assemble to a homotopy pushout square

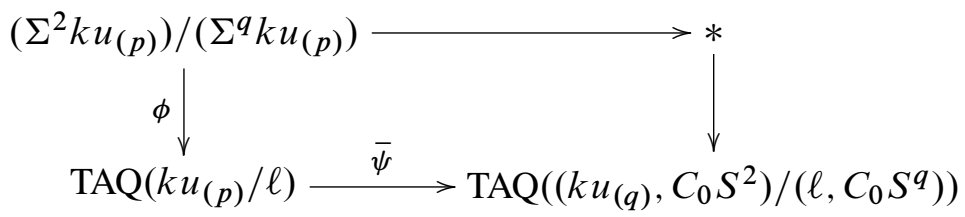

of $k u_{(p)}$-modules. Here

$$
\left(\Sigma^{2} k u_{(p)}\right) /\left(\Sigma^{q} k u_{(p)}\right) \simeq \bigvee_{j=1}^{p-2} \Sigma^{2 j} H \mathbb{Z}_{(p)}
$$


is the homotopy cofiber of the different map $(p-1) u^{p-2} \cdot: k u_{(p)} \wedge S^{q} \rightarrow k u_{(p)} \wedge S^{2}$, while the homotopy cofiber of multiplication by $(p-1)$ is $p$-locally contractible.

Hence the map $\left(f, f^{b}\right):\left(\ell, C_{0} S^{q}, \alpha\right) \rightarrow\left(k u_{(p)}, C_{0} S^{2}, \beta_{(p)}\right)$ is (formally) based log étale if and only if the map

$$
\phi:\left(\Sigma^{2} k u_{(p)}\right) /\left(\Sigma^{q} k u_{(p)}\right) \rightarrow \operatorname{TAQ}\left(k u_{(p)} / \ell\right)=\operatorname{TAQ}^{\ell}\left(k u_{(p)}\right)
$$

(induced by $\alpha: \Sigma^{\bullet} C_{0} S^{q} \rightarrow \ell$ and $\left.\beta_{(p)}: \Sigma^{\bullet} C_{0} S^{2} \rightarrow k u_{(p)}\right)$ is a weak equivalence.

Lemma 12.15 Let $\left(e, e^{b}\right):(R, Q) \rightarrow(A, N)$ be a map of conically based pre-log symmetric ring spectra, and let $C=R \wedge_{\Sigma^{\bullet} Q} \Sigma^{\bullet} N$, so that the left hand square is a pushout in the following diagram

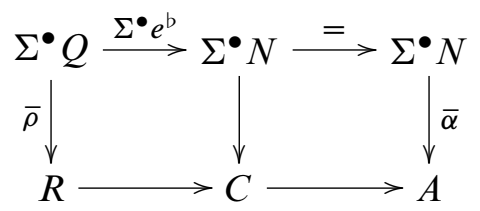

of commutative symmetric ring spectra. Then there is a natural homotopy cofiber sequence

$$
A \wedge\left(B^{\infty} N^{\prime} / B^{\infty} Q^{\prime}\right) \stackrel{\bar{\phi}}{\rightarrow} \mathrm{TAQ}_{0}^{(R, Q)}(A, N) \rightarrow \mathrm{TAQ}^{C}(A)
$$

of $A$-modules. Hence $(R, Q) \rightarrow(A, N)$ is formally based log étale if and only if the connecting map

$$
\text { ว: } \operatorname{TAQ}^{C}(A) \rightarrow \Sigma A \wedge\left(B^{\infty} N^{\prime} / B^{\infty} Q^{\prime}\right)
$$

is an equivalence.

Proof The proof is practically identical to that of Lemma 11.25.

Example 12.16 We apply Lemma 12.15 to the map $\left(\ell, C_{0} S^{q}\right) \rightarrow\left(k u_{(p)}, C_{0} S^{2}\right)$. In this case $S \simeq B^{\infty} C_{0}\left(S^{q}\right)^{\prime} \rightarrow B^{\infty} C_{0}\left(S^{2}\right)^{\prime} \simeq S$ is multiplication by $(p-1)$, which is a $p$-local equivalence. Hence the target $\Sigma A \wedge\left(B^{\infty} N^{\prime} / B^{\infty} Q^{\prime}\right)$ of $\partial$ is contractible, and $\left(f, f^{\mathrm{b}}\right)$ is formally based log étale only if the map

$$
C=\ell \wedge_{\Sigma} C_{0} S^{q} \Sigma^{\bullet} C_{0} S^{2} \rightarrow k u_{(p)}=A
$$

is formally étale. Now both $C$ and $A$ are connective, and the map is a $\pi_{0}$-isomorphism, so this will only happen if $C \rightarrow A$ is an equivalence. See Basterra [12, Lemma 8.2], or the corrected statement in Baker-Gilmour-Reinhard [11, Lemma 1.2]. However, $C$ and $A$ are not equivalent. 
To see this, consider the base change along $R=\ell \rightarrow H=H \mathbb{F}_{p}$. If $C \rightarrow A$ is an equivalence, then so is

$$
H \wedge \Sigma^{\bullet} C_{0} S^{q} \Sigma^{\bullet} C_{0} S^{2} \rightarrow H \wedge_{\ell} k u_{(p)}
$$

where the right hand side has homotopy $P_{p-1}(u)=P(u) /\left(u^{p-1}\right)$ concentrated in even dimensions $0 \leq * \leq(2 p-4)$. The homotopy of the left hand side can be computed by the Künneth spectral sequence

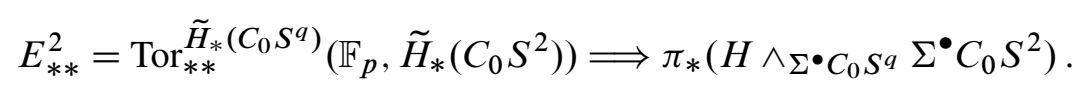

By the Snaith splitting, $\widetilde{H}_{*}\left(C_{0} S^{q}\right) \cong H_{*}\left(C S^{q}\right)$ can be computed as in Cohen-LadaMay [18, I.4.1]. It is isomorphic to the free graded commutative algebra on generators

$$
\iota_{q}, \beta Q^{p} \iota_{q}, Q^{p} \iota_{q}, \ldots
$$

in dimensions $q=2 p-2,2 p^{2}-3,2 p^{2}-2$, etc. Similarly, $\widetilde{H}_{*}\left(C_{0} S^{2}\right) \cong H_{*}\left(C S^{2}\right)$ is isomorphic to the free graded commutative algebra on generators

$$
\iota_{2}, \beta Q^{2} \iota_{2}, Q^{2} \iota_{2}, \ldots
$$

in dimensions $2,4 p-3,4 p-4$, etc. Hence, in dimensions $*<\left(2 p^{2}-3\right)$ the algebra $\tilde{H}_{*}\left(C_{0} S^{q}\right)$ agrees with $P\left(\iota_{q}\right)$, and $\widetilde{H}_{*}\left(C_{0} S^{2}\right)$ is flat over $P\left(\iota_{q}\right)$. In this range of dimensions the spectral sequence is therefore concentrated on the vertical axis, where in addition to the terms $P\left(\iota_{2}\right) \otimes_{P\left(\iota_{q}\right)} \mathbb{F}_{p}=P_{p-1}\left(\iota_{2}\right)$ there are further terms, starting with $\mathbb{F}_{p}\left\{\beta Q^{2} \iota_{2}\right\}$ in dimension ( $\left.4 p-3\right)$. Hence the map $C \rightarrow A=k u_{(p)}$ is precisely $(4 p-3)-$ connected, and is not an equivalence.

Example 12.17 There is an action on the $p$-complete connective $K$-theory spectrum $k u_{p}$ by the group $\Delta \cong \mathrm{GL}_{1}(\mathbb{Z} / p) \cong \mathbb{Z} /(p-1)$ of roots of unity in $\mathbb{Z}_{p}$, with $k \in \Delta$ acting as the $p$-adic Adams operation $\psi^{k}$. The map $f: \ell_{p} \rightarrow k u_{p}$ identifies the $p-$ complete Adams summand with the homotopy fixed points $k u_{p}^{h \Delta}$ of this action. There is a similar $\Delta$-action on the $p$-complete sphere $S_{p}^{2}$, and the map $f^{\mathrm{b}}: C_{0} S_{p}^{q} \rightarrow C_{0} S_{p}^{2}$ factors through the homotopy fixed points

$$
M=\left(C_{0} S_{p}^{2}\right)^{h \Delta} \simeq \bigvee_{(p-1) \mid j \geq 0}\left(S_{p}^{2 j}\right)_{h \Sigma_{j}} .
$$

Furthermore, the pre-log structure $\left(C_{0} S_{p}^{q}, \alpha_{p}\right)$ on $\ell_{p}$ factors through a pre-log structure $\left(M, \beta_{p}^{h \Delta}\right)$, where $\beta_{p}^{h \Delta}$ is given as the $\Delta$-homotopy fixed points of the map

$$
\beta_{p}: N=C_{0} S_{p}^{2} \rightarrow \Omega^{\bullet} k u_{p}
$$


Now $M^{\prime} \simeq \bigsqcup_{(p-1) \mid j \geq 0} B \Sigma_{j}$ maps to $N^{\prime} \simeq \bigsqcup_{j \geq 0} B \Sigma_{j}$ by the inclusion, which identifies $B^{\infty} M^{\prime}$ with the subspectrum of $B^{\infty} N^{\prime} \simeq S$ such that $\Omega^{\infty} B^{\infty} M^{\prime} \simeq$ $\amalg_{(p-1) \mid j} Q_{j}\left(S^{0}\right) \subset Q\left(S^{0}\right)=\Omega^{\infty} S$. Hence $B^{\infty} M^{\prime} \rightarrow B^{\infty} N^{\prime}$ becomes an equivalence after smashing with $k u_{p}$, so

$$
\left(\ell_{p}, M\right)=\left(\ell_{p},\left(C_{0} S_{p}^{2}\right)^{h \Delta}\right) \rightarrow\left(k u_{p}, C_{0} S_{p}^{2}\right)=\left(k u_{p}, N\right)
$$

is formally based log étale only if the map

$$
D=\ell_{p} \wedge_{\Sigma}^{\bullet} M \Sigma^{\bullet} N \rightarrow k u_{p}
$$

is an equivalence. As in Example 12.16, we are led to calculate the maps

$$
H_{*}\left(C S^{q}\right) \rightarrow H_{*}\left(C S^{2}\right)^{\Delta} \rightarrow H_{*}\left(C S^{2}\right)
$$

in a range of dimensions. Here $\widetilde{H}_{*}(N)=H_{*}\left(C S^{2}\right)^{\Delta}$ agrees with $P\left(\left(\iota_{2}\right)^{p-1}\right)$ up to dimension $(6 p-7)$, where a new class $\iota_{2}^{p-2} \cdot \beta Q^{2} \iota_{2}$ enters. For $p \geq 5$ this range of dimensions contains the extra class $\beta Q^{2} \iota_{2}$ in $H_{*}\left(C S^{2}\right)$ that contributes to $\pi_{*}(D)$, so $D \rightarrow k u_{p}$ is also precisely $(4 p-3)-$ connected, and is not an equivalence.

Remark 12.18 The last two examples show that neither $\left(\ell, C_{0} S^{q}\right) \rightarrow\left(k u_{(p)}, C_{0} S^{2}\right)$ nor $\left(\ell_{p},\left(C_{0} S_{p}^{2}\right)^{h \Delta}\right) \rightarrow\left(k u_{p}, C_{0} S^{2}\right)$ are formally based log étale. On the other hand, Ausoni [4, Section 10] has shown that when $\operatorname{THH}\left(\ell_{p} \mid L_{p}\right)$ and $\operatorname{THH}\left(k u_{p} \mid K U_{p}\right)$ are defined so as to sit in homotopy cofiber sequences

$$
\begin{aligned}
& \operatorname{THH}\left(\mathbb{Z}_{p}\right) \stackrel{\pi_{*}}{\longrightarrow} \operatorname{THH}\left(\ell_{p}\right) \stackrel{\rho^{*}}{\rightarrow} \operatorname{THH}\left(\ell_{p} \mid L_{p}\right) \\
& \operatorname{THH}\left(\mathbb{Z}_{p}\right) \stackrel{\pi_{*}}{\longrightarrow} \operatorname{THH}\left(k u_{p}\right) \stackrel{\rho^{*}}{\longrightarrow} \operatorname{THH}\left(k u_{p} \mid K U_{p}\right)
\end{aligned}
$$

of spectra, where the two maps labeled $\pi_{*}$ are transfer maps, then there is an equivalence

$$
k u_{p} \wedge_{\ell_{p}} \operatorname{THH}\left(\ell_{p} \mid L_{p}\right) \stackrel{\simeq}{\rightarrow} \operatorname{THH}\left(k u_{p} \mid K U_{p}\right) .
$$

If there are conically based pre-log structures $M$ and $N$ on $\ell_{p}$ and $k u_{p}$, respectively, such that

$$
\begin{aligned}
\operatorname{THH}\left(\ell_{p} \mid L_{p}\right) & \simeq \operatorname{THH}\left(\ell_{p}, M\right) \\
\operatorname{THH}\left(k u_{p} \mid K U_{p}\right) & \simeq \operatorname{THH}\left(k u_{p}, N\right),
\end{aligned}
$$

then this equivalence is effectively equivalent to the condition that $\left(\ell_{p}, N\right) \rightarrow\left(k u_{p}, M\right)$ is formally based log étale.

Examples 12.16 and 12.17 show that this is not the case for the free commutative based pre-log structure $N=C_{0} S^{2}$ on $k u_{p}$ that is generated by the Bott class $u: S^{2} \rightarrow k u_{p}$, 
when $M$ is either $C_{0} S^{q}$ or $\left(C_{0} S^{2}\right)^{h \Delta}$. This does not exclude the possibility that a conically based pre-log structure $N$ on $k u_{p}$ with $\operatorname{THH}\left(k u_{p} \mid K U_{p}\right) \simeq \operatorname{THH}\left(k u_{p}, N\right)$ exists, but if it does, it will require more (free commutative) cells than the single one generated by the Bott class. The calculations above suggest that the next cell needed is a $(4 p-2)$-cell, attached to cancel $\beta Q^{2} \iota_{2}$.

The search for a suitable log structure $N$ on $k u_{p}$ seems to be related to the question of how to present $H \mathbb{Z}_{p}$ as a commutative $k u_{p}$-algebra. One possibility is that $N$ should be built as a CW commutative conically based $\mathcal{I}$-space monoid, with cells in one-to-one correspondence with a model for $H \mathbb{Z}_{p}$ as a $\mathrm{CW}$ commutative $k u_{p}$-algebra.

\section{Shear maps and repletion}

Definition 13.1 Let $M$ be a commutative monoid. We view the diagonal map

$$
\Delta: M \rightarrow M \times M
$$

as a map of commutative monoids over $M$, where the source is augmented by the identity map $M \rightarrow M$, and the target is augmented by the projection $\mathrm{pr}_{1}: M \times M \rightarrow M$ to the first factor. For reasons related to the cyclic structures discussed in Remark 3.18, we compose the diagonal map with the group completion map

$$
\text { id } \times \gamma: M \times M \rightarrow M \times M^{\mathrm{gp}}
$$

in the second factor. This target is also augmented by the projection $\mathrm{pr}_{1}: M \times M^{\mathrm{gp}} \rightarrow M$ to the first factor, and id $\times \gamma$ is a map of commutative monoids over $M$. The extension of the composite map (id $\times \gamma) \Delta$ to a map of commutative monoids under and over $M$ is the shear map

$$
\text { sh: } M \times M \stackrel{\operatorname{id} \times \Delta}{\longrightarrow} M \times M \times M \stackrel{\mu \times \gamma}{\longrightarrow} M \times M^{\mathrm{gp}}
$$

given by $\operatorname{sh}(x, y)=(x y, \gamma(y))$, where $\mu$ is the multiplication map. Both the source and target are commutative monoids under $M$, by the inclusions in $_{1}: M \rightarrow M \times M$ and $\operatorname{in}_{1}: M \rightarrow M \times M^{\mathrm{gp}}$ on the respective first factors. The source is augmented over $M$ by $\mu$, and the target is augmented over $M$ by $\operatorname{pr}_{1}$.

Passing to monoid rings, there is a shear map

$$
\psi=\mathbb{Z}[\mathrm{sh}]: \mathbb{Z}[M] \otimes \mathbb{Z}[M] \rightarrow \mathbb{Z}[M] \otimes \mathbb{Z}\left[M^{\mathrm{gp}}\right]
$$

of augmented commutative $\mathbb{Z}[M]$-algebras, given by linearly extending the formula $\psi(x, y)=(x y, \gamma(y))$ for $x, y \in M$. On both sides the $\mathbb{Z}[M]$-algebra unit is the inclusion on the first tensor factor, the source is augmented by the ring multiplication, 
and the target is augmented by the projection $\mathbb{Z}[M] \otimes \mathbb{Z}\left[M^{\mathrm{gp}}\right] \rightarrow \mathbb{Z}[M] \otimes \mathbb{Z} \cong \mathbb{Z}[M]$ induced by $M^{\mathrm{gp}} \rightarrow *$. Either shear map is an isomorphism if and only if $M$ is an abelian group.

Definition 13.2 The category $M / \mathcal{C}$ Mon $/ M$ of commutative monoids under and over $M$ has tensor products with based sets, where $Y \widetilde{\otimes}_{M} N$ is the base change of the $Y$ fold coproduct $\bigoplus_{M}^{Y} N=N \oplus_{M} \cdots \oplus_{M} N$ along the augmentation $N \rightarrow M$ in the base point summand. Hence the category of simplicial objects in $M / \mathcal{C M o n} / M$ has tensor products $Y \widetilde{\otimes}_{M} N$ with based simplicial sets. In the case of the circle $S^{1}=\Delta^{1} / \partial \Delta^{1}$ we obtain the suspension $S^{1} \widetilde{\otimes}_{M} N$ in this category, and more generally, tensor product with the $n$-sphere $S^{n}$ realizes $n$-fold suspension $S^{n} \widetilde{\otimes}_{M} N$ in this category.

Lemma 13.3 The suspension

$$
S^{1} \widetilde{\otimes}_{M}(M \times M, \mu) \cong B^{\mathrm{cy}} M
$$

of $M \times M$ augmented by $\mu$ is the cyclic bar construction, whereas the suspension

$$
S^{1} \widetilde{\otimes}_{M}\left(M \times M^{\mathrm{gp}}, \mathrm{pr}_{1}\right) \cong M \times B M^{\mathrm{gp}}
$$

of $M \times M^{\mathrm{gp}}$ augmented by $\mathrm{pr}_{1}$ is $M$ times the suspension of $M^{\mathrm{gp}}$ in simplicial commutative monoids, ie, the bar construction $B M^{\mathrm{gp}}$. The suspension of the shear map

$$
S^{1} \widetilde{\otimes}_{M} \text { sh: } B^{\text {cy }} M \rightarrow M \times B M^{\text {gp }} \cong B^{\text {rep }} M
$$

equals the composite of

$$
(\epsilon, \pi): B^{\mathrm{cy}} M \stackrel{\Delta}{\rightarrow} B^{\mathrm{cy}} M \times B^{\mathrm{cy}} M \stackrel{\epsilon \times \pi}{\longrightarrow} M \times B M
$$

with the weak equivalence id $\times B \gamma: M \times B M \rightarrow M \times B M^{\mathrm{gp}}$. It takes the $q$-simplex $\left(m_{0}, m_{1}, \ldots, m_{q}\right)$ to the pair consisting of $m_{0} m_{1} \cdots m_{q}$ and $\left[\gamma\left(m_{1}\right)|\cdots| \gamma\left(m_{q}\right)\right]$. Hence $S^{1} \widetilde{\otimes}_{M}$ sh equals the repletion map $B^{\text {cy }} M \rightarrow B^{\text {rep }} M$.

The suspension in $\mathbb{Z}[M] / \mathcal{C R}$ ing $/ \mathbb{Z}[M]$ takes the shear map

$$
\psi: \mathbb{Z}[M] \otimes \mathbb{Z}[M] \rightarrow \mathbb{Z}[M] \otimes \mathbb{Z}\left[M^{\mathrm{gp}}\right]
$$

to the corepresenting map

$$
\psi=\psi^{[1]}: \operatorname{HH}(\mathbb{Z}[M])=\mathbb{Z}\left[B^{\mathrm{cy}} M\right] \rightarrow \mathbb{Z}\left[M \times B M^{\mathrm{gp}}\right] \cong \mathbb{Z}\left[B^{\mathrm{rep}} M\right]
$$

from Remark 3.24 and Lemma 5.24, where $\mathbb{Z}\left[B^{\text {rep }} M\right]=\mathrm{HH}(\mathbb{Z}[M], M)$. 
Definition 13.4 The Hochschild homology of $\mathbb{Z}[M]$, the log Hochschild homology of $(\mathbb{Z}[M], M)$, and the repletion homomorphism

$$
\psi: \mathrm{HH}(\mathbb{Z}[M]) \rightarrow \mathrm{HH}(\mathbb{Z}[M], M)
$$

can be (re-)defined as the suspension in augmented commutative $\mathbb{Z}[M]$-algebras of the shear map $\mathbb{Z}[M] \otimes \mathbb{Z}[M] \rightarrow \mathbb{Z}[M] \otimes \mathbb{Z}\left[M^{\mathrm{gp}}\right]$.

The log Hochschild homology of a general pre-log ring $(A, M, \alpha)$ is (re-)defined by the homotopy pushout

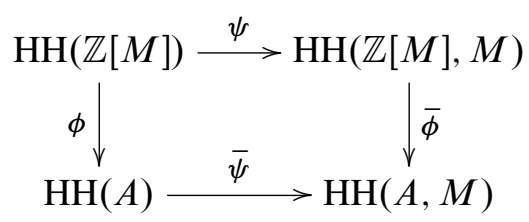

in simplicial commutative rings, where $\phi$ is induced by the pre-log structure map $\bar{\alpha}: \mathbb{Z}[M] \rightarrow A$.

Lemma 13.5 For $n \geq 1$, the $n$-fold suspension

$$
S^{n} \tilde{\otimes}_{M}(M \times M, \mu) \cong S^{n} \otimes M
$$

is the $n$-th order cyclic bar construction on $M$,

$$
S^{n} \widetilde{\otimes}_{M}\left(M \times M^{\mathrm{gp}}, \mathrm{pr}_{1}\right) \cong M \times B^{n} M^{\mathrm{gp}}
$$

is $M$ times the $n$-fold bar construction on $M^{\mathrm{gp}}$, and the $n$-th suspension of the shear map

$$
S^{n} \widetilde{\otimes}_{M} \text { sh: } S^{n} \otimes M \rightarrow M \times B^{n} M^{\mathrm{gp}} \cong S^{n} \otimes^{\text {rep }} M
$$

equals the repletion map.

Definition 13.6 The $n$-fold suspension in $\mathbb{Z}[M] / \mathcal{C} \mathcal{R} i n g / \mathbb{Z}[M]$ takes the shear map to the repletion map

$$
\psi=\psi^{[n]}: \mathrm{HH}^{[n]}(\mathbb{Z}[M])=\mathbb{Z}\left[S^{n} \otimes M\right] \rightarrow \mathbb{Z}\left[M \times B^{n} M^{\mathrm{gp}}\right]=\mathrm{HH}^{[n]}(\mathbb{Z}[M], M)
$$

from Pirashvili's $n$-th order Hochschild homology of $\mathbb{Z}[M]$ [56, Section 5.1] to an $n$-th order log Hochschild homology of $(\mathbb{Z}[M], M)$. In general, the $n$-th order log Hochschild homology $\mathrm{HH}^{[n]}(A, M)$ of $(A, M)$ is defined by a homotopy pushout of simplicial commutative rings, like that in Definition 13.4. 
Remark 13.7 Robinson and Whitehouse [63] defined $\Gamma$-homology groups $H \Gamma_{*}(A)$, which are the $E_{\infty}$ DGA analogue of the André-Quillen homology groups $D_{*}(A)$ for commutative (simplicial) rings. In particular, $H \Gamma_{0}(A) \cong D_{0}(A) \cong \Omega_{A}^{1}$. By a theorem of Pirashvili and Richter [57, Theorem 1], the groups $\mathrm{HH}_{*+n}^{[n]}(A)$ stabilize to the $\Gamma$-homology groups $H \Gamma_{*}(A)$ when $n \rightarrow \infty$. Hence stabilization of higher order Hochschild homology does not quite give André-Quillen homology in the context of commutative rings (unless $A$ is a $\mathbb{Q}$-algebra), but its $E_{\infty}$ DGA analogue. In the topological setting there is no essential difference between $E_{\infty}$ ring spectra and commutative $S$-algebras, and stabilization of higher order topological Hochschild homology does, indeed, give topological André-Quillen homology, as proved by Basterra and Mandell [13, Theorem 4]. See Proposition 13.12 below.

Definition 13.8 Let $M$ be a commutative $\mathcal{I}$-space monoid, with group completion $\Gamma M$. There is a chain of maps

$$
M \otimes M \stackrel{\operatorname{id} \otimes \Delta}{\longrightarrow} M \otimes M \times M \stackrel{\simeq}{\longleftarrow} M \otimes M \otimes M \stackrel{\mu \otimes \gamma}{\longrightarrow} M \otimes \Gamma M
$$

where the middle map is a weak equivalence for reasonable (cofibrant and semistable) $M$. These are maps of commutative $\mathcal{I}$-space monoids under and over $M$, where the left hand $M \otimes M$ is augmented by the commutative monoid multiplication $\mu$, and the right hand $M \otimes \Gamma M$ is augmented by the projection $M \otimes \Gamma M \rightarrow M \otimes * \cong M$.

There is a chain of maps

$$
\begin{aligned}
\psi: S[M] \wedge S[M] \stackrel{\operatorname{id} \wedge S[\Delta]}{\longrightarrow} S[M] & \wedge S[M \times M] \\
& \stackrel{\simeq}{\longleftarrow} S[M] \wedge S[M] \wedge S[M] \stackrel{\mu \wedge \gamma}{\longrightarrow} S[M] \wedge S[\Gamma M]
\end{aligned}
$$

of augmented commutative $S[M]$-algebras, with augmentations induced from those in the commutative $\mathcal{I}$-space monoid case.

Lemma 13.9 The suspension

$$
S^{1} \tilde{\otimes}_{S[M]}(S[M] \wedge S[M], \mu) \cong S^{1} \otimes S[M]=\mathrm{THH}(S[M])
$$

of $S[M] \wedge S[M]$ augmented by $\mu$ is the topological Hochschild homology of $S[M]$. The suspension

$$
S^{1} \widetilde{\otimes}_{S[M]}\left(S[M] \wedge S[\Gamma M], \mathrm{pr}_{1}\right) \simeq S[M] \wedge B \Gamma M_{+}=\mathrm{THH}(S[M], M)
$$

of $S[M] \wedge S[\Gamma M]$ augmented by $\mathrm{pr}_{1}$ is the $S[M]$-module extended up from the bar construction $B(S, S[\Gamma M], S) \cong S[B \Gamma M]$. Here $\operatorname{THH}(S[M], M) \cong S\left[B^{\text {rep }} M\right]$ is the 
$\log$ topological Hochschild homology of $(S[M], M)$. The suspension of the shear map $\psi$ is the corepresenting map

$$
\psi=\psi^{[1]}: \operatorname{THH}(S[M]) \rightarrow \operatorname{THH}(S[M], M)
$$

from Definition 8.11.

Proof This is clear by a comparison with Definition 8.10.

Definition 13.10 The topological Hochschild homology of $S[M]$, the log topological Hochschild homology of $(S[M], M)$, and the repletion map

$$
\psi: \operatorname{THH}(S[M]) \rightarrow \operatorname{THH}(S[M], M)
$$

can be (re-)defined as the suspension in commutative augmented $S[M]$-algebras of the shear map

$$
\psi: S[M] \wedge S[M] \rightarrow S[M] \wedge S[\Gamma M] .
$$

More precisely, the shear map is a chain of maps, and the repletion map is the suspended chain of maps. The log topological Hochschild homology of a pre-log symmetric ring spectrum $(A, M, \alpha)$ is defined by the homotopy pushout

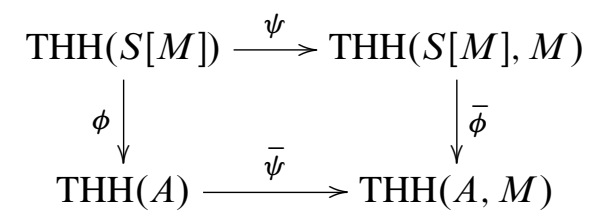

of commutative symmetric ring spectra, where $\phi$ is induced by $\bar{\alpha}: S[M] \rightarrow A$.

Definition 13.11 For $n \geq 1$, the $n$-th order topological Hochschild homology

$$
\mathrm{THH}^{[n]}(S[M])=S^{n} \tilde{\otimes}_{S[M]}(S[M] \wedge S[M], \mu)
$$

of $S[M]$, the $n$-th order log topological Hochschild homology

$$
\mathrm{THH}^{[n]}(S[M], M)=S^{n} \tilde{\otimes}_{S[M]}\left(S[M] \wedge S[\Gamma M], \mathrm{pr}_{1}\right) \cong S[M] \wedge B^{n} \Gamma M_{+}
$$

of $(S[M], M)$, and the repletion map

$$
\psi=\psi^{[n]}: \mathrm{THH}^{[n]}(S[M]) \rightarrow \mathrm{THH}^{[n]}(S[M], M)
$$

are defined as the $n$-fold suspensions in commutative augmented $S[M]$-algebras of the source and target of the shear map, and the shear map itself. For general $(A, M, \alpha)$, the $n$-th order log topological Hochschild homology $\operatorname{THH}^{[n]}(A, M)$ is defined by a homotopy pushout, like in Definition 13.10. 
Proposition 13.12 The stabilization as $n \rightarrow \infty$ of the repletion map $\psi^{[n]}$ in $n$-th order topological Hochschild homology is the corepresenting map

$$
\psi=\psi^{[\infty]}: \operatorname{TAQ}(S[M]) \rightarrow \operatorname{TAQ}(S[M], M)=S[M] \wedge B^{\infty} \Gamma M
$$

in topological André-Quillen homology, from Lemma 11.16. This equals the map of commutative $S[M]$-algebra indecomposables

$$
\begin{aligned}
\mathbb{L A b}_{S[M]}^{S[M]}(S[M] \wedge S[M], \mu) & \\
& \rightarrow \mathbb{L A b}{ }_{S[M]}^{S[M]}\left(S[M] \wedge S[\Gamma M], \operatorname{pr}_{1}\right) \simeq S[M] \wedge \mathbb{L A} \mathrm{Lb}_{S}^{S} S[\Gamma M]
\end{aligned}
$$

induced by the shear map $\psi$.

Proof (See Basterra-Mandell [13, Section 2] for the definition of the commutative algebra indecomposables functor $\mathbb{L} \mathrm{Ab}$.) Let $\overline{\mathrm{THH}}^{[n]}(A)$ be the (homotopy) cofiber of the unit $\eta: A \rightarrow \mathrm{THH}^{[\eta]}(A)$. The sequence

$$
\left\{n \mapsto \overline{\mathrm{THH}}^{[n]}(A)\right\}
$$

defines a spectrum of $A$-modules. The category of $A$-modules is already stable, so this spectrum corresponds to the $A$-module given by the homotopy colimit

$$
\underset{n}{\operatorname{hocolim}} \Sigma^{-n} \overline{\mathrm{THH}}^{[n]}(A) \text {. }
$$

By [13, Theorem 4], this homotopy colimit is equivalent to the commutative $A$-algebra indecomposables $\mathbb{L A b}_{A}^{A}(A \wedge A, \mu) \simeq \mathrm{TAQ}(A)$, as an $A$-module. In the special case $A=S[M]$, this gives the claim for $\operatorname{TAQ}(S[M])$.

It is clear that the spectrum

$$
\left\{n \mapsto \overline{\mathrm{THH}}^{[n]}(S[M], M) \cong S[M] \wedge B^{n} \Gamma M\right\}
$$

stabilizes to $\operatorname{TAQ}(S[M], M)=S[M] \wedge B^{\infty} \Gamma M$, and that this equals

$$
\mathbb{L} \mathrm{Ab}_{S[M]}^{S[M]}\left(S[M] \wedge S[\Gamma M], \operatorname{pr}_{1}\right) \cong S[M] \wedge \mathbb{L} \mathrm{Ab}_{S}^{S} S[\Gamma M] \simeq S[M] \wedge B^{\infty} \Gamma M,
$$

by $[13,6.1]$.

By Proposition 11.17 in the case when $A=S[M]$, we have checked that the corepresenting map agrees with the stabilized shear map, when $M=C X$ is a free commutative $\mathcal{I}$-space monoid. The general comparison result can be deduced from this (modulo coherence issues) by freely resolving a general commutative $\mathcal{I}$-space monoid. For an alternative proof, we can start with the comparison for topological Hochschild homology in Lemma 13.9 and stabilize. 
Definition 13.13 The log topological André-Quillen homology of a pre-log symmetric ring spectrum $(A, M, \alpha)$, denoted $\operatorname{TAQ}(A, M)$, can be (re-)defined as the homotopy pushout

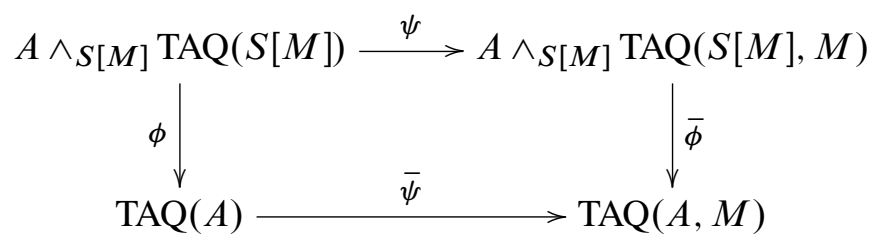

of $A$-modules, where $\psi$ is the stable shear map $\psi^{[\infty]}$ extended along $\bar{\alpha}: S[M] \rightarrow A$, and $\phi$ is induced by $\bar{\alpha}$.

Definition 13.14 Let $N$ be a commutative conically based $\mathcal{I}$-space monoid. Hence $N=\operatorname{cone}(L) \cup_{L} N^{\prime}$, and the multiplication $\mu: N \boxminus N \rightarrow N$ restricts to a multiplication $N^{\prime} \otimes N^{\prime} \rightarrow N^{\prime}$ making $N^{\prime}$ a commutative $\mathcal{I}$-space monoid, with group completion $\Gamma N^{\prime}$. Let

$$
\delta: N \rightarrow N \wedge N_{+}^{\prime}
$$

be the conical diagonal map. This is a map of commutative based $\mathcal{I}$-space monoids over $N$, where the source is augmented by the identity and the target is augmented by the projection $\mathrm{pr}_{1}: N \wedge N_{+}^{\prime} \rightarrow N \wedge *_{+} \cong N$, induced by the unique map $N^{\prime} \rightarrow *$. Over $N^{\prime} \subset N$ the map $\delta$ equals the diagonal map

$$
\delta \mid N^{\prime}: N^{\prime} \stackrel{\Delta}{\rightarrow} N^{\prime} \times N^{\prime} \subset N \wedge N_{+}^{\prime}
$$

and over cone $(L)$ the map $\delta$ is constant in the cone direction.

The based shear map

$$
\text { sh: } N \boxminus N \stackrel{\operatorname{id} \unrhd \delta}{\longrightarrow} N \boxminus N \wedge N_{+}^{\prime} \stackrel{\simeq}{\longleftarrow} N \boxminus N \boxminus N_{+}^{\prime} \stackrel{\mu \wedge \gamma}{\longrightarrow} N \boxminus \Gamma N_{+}^{\prime}
$$

is a chain of maps of commutative $\mathcal{I}$-space monoids under and over $N$. The source $N \oslash N$ is augmented by $\mu$ and the target $N \oslash \Gamma N_{+}^{\prime}$ is augmented by $\operatorname{pr}_{1}$. It induces a chain of maps

$$
\begin{aligned}
\psi: \Sigma^{\bullet} N \wedge \Sigma^{\bullet} N \stackrel{\operatorname{id} \wedge \Sigma^{\bullet} \delta}{\longrightarrow} \Sigma^{\bullet} N \wedge \Sigma^{\bullet} & \left(N \wedge N_{+}^{\prime}\right) \\
& \stackrel{\simeq}{\longleftarrow} \Sigma^{\bullet} N \wedge \Sigma^{\bullet} N \wedge S\left[N^{\prime}\right] \stackrel{\mu \wedge \gamma}{\longrightarrow} \Sigma^{\bullet} N \wedge S\left[\Gamma N^{\prime}\right]
\end{aligned}
$$

of augmented commutative $\Sigma^{\bullet} N$-algebras. 
Definition 13.15 Let $N$ be a commutative conically based $\mathcal{I}$-space monoid. The suspension

$$
S^{1} \widetilde{\otimes}_{\Sigma^{\bullet} N}\left(\Sigma^{\bullet} N \wedge \Sigma^{\bullet} N, \mu\right)=\Sigma^{\bullet} B_{\wedge}^{\mathrm{cy}} N=\operatorname{THH}\left(\Sigma^{\bullet} N\right)
$$

of $\Sigma^{\bullet} N \wedge \Sigma^{\bullet} N$ augmented by $\mu$ is the topological Hochschild homology of $\Sigma^{\bullet} N$. The based log topological Hochschild homology of $\left(\Sigma^{\bullet} N, N\right)$ can be (re-)defined as the suspension

$$
S^{1} \widetilde{\otimes}_{\Sigma^{\bullet} N}\left(\Sigma^{\bullet} N \wedge S\left[\Gamma N^{\prime}\right], \operatorname{pr}_{1}\right)=\Sigma^{\bullet} N \wedge B\left(\Gamma N^{\prime}\right)_{+}=\operatorname{THH}_{0}\left(\Sigma^{\bullet} N, N\right)
$$

of $\Sigma^{\bullet} N \wedge S\left[\Gamma N^{\prime}\right]$ augmented by $\mathrm{pr}_{1}$. The repletion map

$$
\psi=\psi^{[1]}: \operatorname{THH}\left(\Sigma^{\bullet} N\right) \rightarrow \mathrm{THH}_{0}\left(\Sigma^{\bullet} N, N\right)
$$

can be (re-)defined as the suspension of the shear map $\psi$. The based log topological Hochschild homology of a conically based pre-log symmetric ring spectrum $(A, N, \alpha)$ is defined by the homotopy pushout

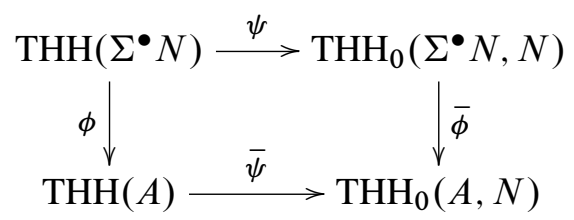

of commutative symmetric ring spectra, where $\phi$ is induced by $\bar{\alpha}: \Sigma^{\bullet} N \rightarrow A$.

Similarly, we define $n$-th order based log topological Hochschild homology of $(A, N)$, denoted $\operatorname{THH}_{0}^{[n]}(A, N)$, by starting with the case $A=\Sigma^{\bullet} N$ and considering the $n$-fold suspension in the category $\Sigma^{\bullet} N / \mathcal{C S} p^{\Sigma} / \Sigma^{\bullet} N$ of (the target of) the shear map $\psi$.

Proposition 13.16 The stabilization as $n \rightarrow \infty$ of the repletion map

$$
\psi^{[n]}=S^{n} \tilde{\otimes}_{\Sigma \cdot N} \psi
$$

is the corepresenting map

$$
\psi=\psi^{[\infty]}: \operatorname{TAQ}\left(\Sigma^{\bullet} N\right) \rightarrow \operatorname{TAQ}_{0}\left(\Sigma^{\bullet} N, N\right)=\Sigma^{\bullet} N \wedge B^{\infty} \Gamma N^{\prime}
$$

in based topological André-Quillen homology, as in Definition 12.6. This equals the map of commutative $\Sigma \bullet N$-algebra indecomposables

$$
\mathbb{L} \operatorname{Ab}_{\Sigma^{\bullet} N}^{\Sigma^{\bullet} N}\left(\Sigma^{\bullet} N \wedge \Sigma^{\bullet} N, \mu\right) \rightarrow \mathbb{L} \operatorname{Ab}_{\Sigma^{\bullet}}^{\Sigma^{\bullet} N}\left(\Sigma^{\bullet} N \wedge S\left[\Gamma N^{\prime}\right], \operatorname{pr}_{1}\right) \simeq \Sigma^{\bullet} N \wedge B^{\infty} \Gamma N^{\prime}
$$

induced by the shear map $\psi$. 
Definition 13.17 The based log topological André-Quillen homology $\mathrm{TAQ}_{0}(A, N)$ of a conically based pre-log symmetric ring spectrum $(A, N, \alpha)$ can be (re-)defined as the homotopy pushout

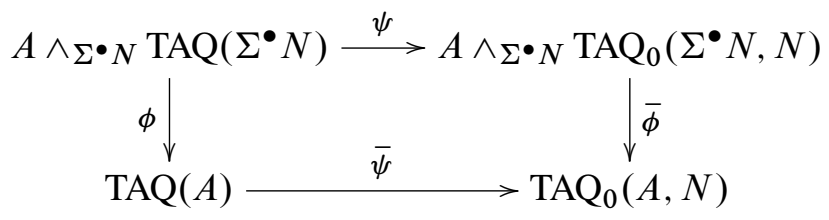

of $A$-modules, where $\psi$ is the stable shear map $\psi^{[\infty]}$ extended along $\bar{\alpha}: \Sigma^{\bullet} N \rightarrow A$, and $\phi$ is induced by $\bar{\alpha}$.

\section{References}

[1] V Angeltveit, Topological Hochschild homology and cohomology of $A_{\infty}$ ring spectra, Geom. Topol. 12 (2008) 987-1032 MR2403804

[2] V Angeltveit, J Rognes, Hopf algebra structure on topological Hochschild homology, Algebr. Geom. Topol. 5 (2005) 1223-1290 MR2171809

[3] C Ausoni, On the algebraic $K$-theory of the complex $K$-theory spectrum arXiv: 0902.2334

[4] C Ausoni, Topological Hochschild homology of connective complex $K$-theory, Amer. J. Math. 127 (2005) 1261-1313 MR2183525

[5] C Ausoni, J Rognes, Algebraic K-theory of topological K-theory, Acta Math. 188 (2002) 1-39 MR1947457

[6] C Ausoni, J Rognes, Rational algebraic $K$-theory of topological $K$-theory arXiv: 0708.2160

[7] C Ausoni, J Rognes, Algebraic $K$-theory of the fraction field of topological $K$-theory, in preparation

[8] N A Baas, B I Dundas, B Richter, J Rognes, Ring completion of rig categories arXiv:0706.0531

[9] N A Baas, B I Dundas, B Richter, J Rognes, Stable bundles over rig categories arXiv:0706.0531

[10] N A Baas, B I Dundas, J Rognes, Two-vector bundles and forms of elliptic cohomology, from: "Topology, geometry and quantum field theory", (U Tillman, editor), London Math. Soc. Lecture Note Ser. 308, Cambridge Univ. Press (2004) 18-45 MR2079370

[11] A Baker, H Gilmour, P Reinhard, Topological André-Quillen homology for cellular commutative $S$-algebras, Abhand. Math. Sem. Univ. Hamburg 78 (2008) 27-50 
[12] M Basterra, André-Quillen cohomology of commutative S-algebras, J. Pure Appl. Algebra 144 (1999) 111-143 MR1732625

[13] M Basterra, M A Mandell, Homology and cohomology of $E_{\infty}$ ring spectra, Math. Z. 249 (2005) 903-944 MR2126222

[14] A J Blumberg, M A Mandell, The localization sequence for the algebraic $K$-theory of topological K-theory, Acta Math. 200 (2008) 155-179 MR2413133

[15] M Bökstedt, W C Hsiang, I Madsen, The cyclotomic trace and algebraic K-theory of spaces, Invent. Math. 111 (1993) 465-539 MR1202133

[16] A K Bousfield, E M Friedlander, Homotopy theory of $\Gamma$-spaces, spectra, and bisimplicial sets, from: "Geometric applications of homotopy theory (Proc. Conf., Evanston, Ill., 1977), II”, Lecture Notes in Math. 658, Springer, Berlin (1978) 80-130 MR513569

[17] M Brun, Z Fiedorowicz, R M Vogt, On the multiplicative structure of topological Hochschild homology, Algebr. Geom. Topol. 7 (2007) 1633-1650 MR2366174

[18] F R Cohen, T J Lada, J P May, The homology of iterated loop spaces, Lecture Notes in Math. 533, Springer, Berlin (1976) MR0436146

[19] F R Cohen, M E Mahowald, R J Milgram, The stable decomposition for the double loop space of a sphere, from: "Algebraic and geometric topology (Proc. Sympos. Pure Math., Stanford Univ., 1976), Part 2", (R J Milgram, editor), Proc. Sympos. Pure Math., XXXII, Amer. Math. Soc. (1978) 225-228 MR520543

[20] W Dwyer, E Friedlander, V Snaith, R Thomason, Algebraic $K$-theory eventually surjects onto topological K-theory, Invent. Math. 66 (1982) 481-491 MR662604

[21] A D Elmendorf, I Kriz, M A Mandell, J P May, Rings, modules, and algebras in stable homotopy theory, Math. Surveys and Monogr. 47, Amer. Math. Soc. (1997) MR1417719 With an appendix by M Cole

[22] F T Farrell, L E Jones, Stable pseudoisotopy spaces of compact non-positively curved manifolds, J. Differential Geom. 34 (1991) 769-834 MR1139646

[23] V Franjou, T Pirashvili, On the Mac Lane cohomology for the ring of integers, Topology 37 (1998) 109-114 MR1480880

[24] E M Friedlander, B Mazur, Filtrations on the homology of algebraic varieties, Mem. Amer. Math. Soc. 110, Amer. Math. Soc. (1994) MR1211371 With an appendix by D Quillen

[25] E Getzler, M M Kapranov, Cyclic operads and cyclic homology, from: "Geometry, topology, \& physics", (S-T Yau, editor), Conf. Proc. Lecture Notes Geom. Topology, IV, Int. Press, Cambridge, MA (1995) 167-201 MR1358617

[26] K Hagihara, Structure theorem of Kummer étale K-group, K-Theory 29 (2003) 75-99 MR2029756

[27] L Hesselholt, On the p-typical curves in Quillen's K-theory, Acta Math. 177 (1996) 1-53 MR1417085 
[28] L Hesselholt, I Madsen, On the K-theory of finite algebras over Witt vectors of perfect fields, Topology 36 (1997) 29-101 MR1410465

[29] L Hesselholt, I Madsen, On the K-theory of local fields, Ann. of Math. (2) 158 (2003) 1-113 MR1998478

[30] L Hesselholt, I Madsen, On the De Rham-Witt complex in mixed characteristic, Ann. Sci. École Norm. Sup. (4) 37 (2004) 1-43 MR2050204

[31] G Hochschild, B Kostant, A Rosenberg, Differential forms on regular affine algebras, Trans. Amer. Math. Soc. 102 (1962) 383-408 MR0142598

[32] M Hovey, B Shipley, J Smith, Symmetric spectra, J. Amer. Math. Soc. 13 (2000) 149-208 MR1695653

[33] L Illusie, Complexe de de Rham-Witt et cohomologie cristalline, Ann. Sci. École Norm. Sup. (4) 12 (1979) 501-661 MR565469

[34] L Illusie, An overview of the work of K Fujiwara, K Kato, and C Nakayama on logarithmic étale cohomology, Cohomologies p-adiques et applications arithmétiques, II, Astérisque (2002) 271-322 MR1922832

[35] K Kato, Logarithmic structures of Fontaine-Illusie, from: "Algebraic analysis, geometry, and number theory (Baltimore, MD, 1988)", (J-I Igusa, editor), Johns Hopkins Univ. Press, Baltimore, MD (1989) 191-224 MR1463703

[36] K Kato, C Nakayama, Log Betti cohomology, log étale cohomology, and log de Rham cohomology of log schemes over C, Kodai Math. J. 22 (1999) 161-186 MR1700591

[37] S O Kochman, Homology of the classical groups over the Dyer-Lashof algebra, Trans. Amer. Math. Soc. 185 (1973) 83-136 MR0331386

[38] A Langer, T Zink, De Rham-Witt cohomology for a proper and smooth morphism, J. Inst. Math. Jussieu 3 (2004) 231-314 MR2055710

[39] L G Lewis, Jr, J P May, M Steinberger, J E McClure, Equivariant stable homotopy theory, Lecture Notes in Math. 1213, Springer, Berlin (1986) MR866482 With contributions by $\mathrm{JE}$ McClure

[40] P Lima-Filho, Completions and fibrations for topological monoids, Trans. Amer. Math. Soc. 340 (1993) 127-147 MR1134758

[41] A Lindenstrauss, I Madsen, Topological Hochschild homology of number rings, Trans. Amer. Math. Soc. 352 (2000) 2179-2204 MR1707702

[42] J-L Loday, Cyclic homology, second edition, Grund. der Math. Wissenschaften 301, Springer, Berlin (1998) MR1600246 Appendix E by MO Ronco, Chapter 13 by the author in collaboration with T Pirashvili

[43] S Lunøe-Nielsen, J Rognes, On the Tate construction of topological Hochschild homology and its relation to the construction of Singer, in preparation 
[44] S Mac Lane, Categories for the working mathematician, second edition, Graduate Texts in Math. 5, Springer, New York (1998) MR1712872

[45] J P May, The geometry of iterated loop spaces, Lectures Notes in Math. 271, Springer, Berlin (1972) MR0420610

[46] J P May, $E_{\infty}$ ring spaces and $E_{\infty}$ ring spectra, Lecture Notes in Math. 577, Springer, Berlin (1977) MR0494077 With contributions by F Quinn, N Ray, and J Tornehave

[47] JP May, What precisely are $E_{\infty}$ ring spaces and $E_{\infty}$ ring spectra?, from: "New topological contexts for Galois theory and algebraic geometry (BIRS 2008)", (A Baker, B Richter, editors), Geom. Topol. Monogr. 16 (2009) 215-282

[48] R McCarthy, V Minasian, HKR theorem for smooth $S$-algebras, J. Pure Appl. Algebra 185 (2003) 239-258 MR2006429

[49] J E McClure, R E Staffeldt, On the topological Hochschild homology of bu. I, Amer. J. Math. 115 (1993) 1-45 MR1209233

[50] JS Milne, Arithmetic duality theorems, Perspectives in Math. 1, Academic Press, Boston (1986) MR881804

[51] V Minasian, André-Quillen spectral sequence for THH, Topology Appl. 129 (2003) 273-280 MR1962984

[52] C Nakayama, Logarithmic étale cohomology, Math. Ann. 308 (1997) 365-404 MR1457738

[53] W Niziol, $K$-theory of log-schemes. I, Doc. Math. 13 (2008) 505-551 MR2452875

[54] M C Olsson, Logarithmic geometry and algebraic stacks, Ann. Sci. École Norm. Sup. (4) 36 (2003) 747-791 MR2032986

[55] M C Olsson, The logarithmic cotangent complex, Math. Ann. 333 (2005) 859-931 MR2195148

[56] T Pirashvili, Hodge decomposition for higher order Hochschild homology, Ann. Sci. École Norm. Sup. (4) 33 (2000) 151-179 MR1755114

[57] T Pirashvili, B Richter, Robinson-Whitehouse complex and stable homotopy, Topology 39 (2000) 525-530 MR1746906

[58] D Puppe, Homotopie und Homologie in abelschen Gruppen- und Monoidkomplexen. I. II, Math. Z. 68 (1958) 367-406, 407-421 MR0093761

[59] D G Quillen, Homotopical algebra, Lecture Notes in Math. 43, Springer, Berlin (1967) MR0223432

[60] D Quillen, On the (co-) homology of commutative rings, from: "Applications of Categorical Algebra (Proc. Sympos. Pure Math., Vol. XVII, New York, 1968)”, Amer. Math. Soc. (1970) 65-87 MR0257068 
[61] D Quillen, Higher algebraic $K$-theory. I, from: "Algebraic $K$-theory, I: Higher $K$ theories (Proc. Conf., Battelle Memorial Inst., Seattle, Wash., 1972)", (H Bass, editor), Lecture Notes in Math. 341, Springer, Berlin (1973) 85-147 MR0338129

[62] D Quillen, Higher algebraic K-theory, from: "Proceedings of the International Congress of Mathematicians (Vancouver, B. C., 1974), Vol. 1", Canad. Math. Congress, Montreal (1975) 171-176 MR0422392

[63] A Robinson, $\mathbf{S}$ Whitehouse, Operads and $\Gamma$-homology of commutative rings, Math. Proc. Cambridge Philos. Soc. 132 (2002) 197-234 MR1874215

[64] J Rognes, Two-primary algebraic K-theory of pointed spaces, Topology 41 (2002) 873-926 MR1923990

[65] J Rognes, The smooth Whitehead spectrum of a point at odd regular primes, Geom. Topol. 7 (2003) 155-184 MR1988283

[66] J Rognes, C Weibel, Two-primary algebraic $K$-theory of rings of integers in number fields, J. Amer. Math. Soc. 13 (2000) 1-54 MR1697095 Appendix A by M Kolster

[67] S Sagave, C Schlichtkrull, Diagram spaces and symmetric spectra, in preparation

[68] C Schlichtkrull, Units of ring spectra and their traces in algebraic $K$-theory, Geom. Topol. 8 (2004) 645-673 MR2057776

[69] C Schlichtkrull, The homotopy infinite symmetric product represents stable homotopy, Algebr. Geom. Topol. 7 (2007) 1963-1977 MR2366183

[70] C Schlichtkrull, Thom spectra that are symmetric spectra arXiv:0811.0592

[71] C Schlichtkrull, Group completion in $\mathcal{I}$-spaces, in preparation

[72] S Schwede, Spectra in model categories and applications to the algebraic cotangent complex, J. Pure Appl. Algebra 120 (1997) 77-104 MR1466099

[73] S Schwede, An untitled book project about symmetric spectra, Manuscript (2007) Available at http://www.math.uni-bonn.de/people/schwede/SymSpec.pdf

[74] G Segal, Configuration-spaces and iterated loop-spaces, Invent. Math. 21 (1973) 213221 MR0331377

[75] J-P Serre, Local fields, Graduate Texts in Math. 67, Springer, New York (1979) MR554237 Translated from the French by M J Greenberg

[76] V P Snaith, A stable decomposition of $\Omega^{n} S^{n} X$, J. London Math. Soc. (2) 7 (1974) 577-583 MR0339155

[77] N P Strickland, Realising formal groups, Algebr. Geom. Topol. 3 (2003) 187-205 MR1997319

[78] $\mathbf{R}$ W Thomason, $\mathbf{T}$ Trobaugh, Higher algebraic $K$-theory of schemes and of derived categories, from: "The Grothendieck Festschrift, Vol. III", (P Cartier, L Illusie, N M Katz, G Laumon, Y Manin, K A Ribet, editors), Progr. Math. 88, Birkhäuser, Boston (1990) 247-435 MR1106918 
[79] F Waldhausen, Algebraic K-theory of topological spaces. II, from: "Algebraic topology, Aarhus 1978 (Proc. Sympos., Univ. Aarhus, 1978)", (J L Dupont, I Madsen, editors), Lecture Notes in Math. 763, Springer, Berlin (1979) 356-394 MR561230

[80] F Waldhausen, Algebraic K-theory of spaces, from: "Algebraic and geometric topology (New Brunswick, N.J., 1983)", (A Ranicki, N Levitt, F Quinn, editors), Lecture Notes in Math. 1126, Springer, Berlin (1985) 318-419 MR802796

[81] F Waldhausen, B Jahren, J Rognes, Spaces of PL manifolds and categories of simple maps, Preprint Available at http://folk.uio.no/rognes/papers/plmf.pdf

[82] A Yamaguchi, Morava K-theory of double loop spaces of spheres, Math. Z. 199 (1988) 511-523 MR968317

Department of Mathematics, University of Oslo

Norway

rognes@math .uio.no

http://folk.uio.no/rognes/

Received: 15 December 2008 